\title{
A SURFACTANT-FREE STRATEGY FOR SYNTHESIZING AND PROCESSING INTERMETALLIC PLATINUM-BASED NANOPARTICLE CATALYSTS
}

\author{
A Dissertation \\ Presented to the Faculty of the Graduate School \\ of Cornell University \\ In Partial Fulfillment of the Requirements for the Degree of \\ Doctor of Philosophy
}

by

HAO CHEN

AUGUST 2013 
(C) 2013 Hao Chen 


\title{
A SURFACTANT-FREE STRATEGY FOR SYNTHESIZING AND PROCESSING INTERMETALLIC PLATINUM-BASED NANOPARTICLE CATALYSTS
}

\author{
Hao Chen, Ph. D. (D.M.A.) (J.S.D)
}

Cornell University 2013

In this thesis, a surfactant-free $\mathrm{Np}-\mathrm{KCl}$ matrix method ( $\mathrm{Np}$ stands for nanoparticle, $\mathrm{KCl}$ is potassium chloride) is developed for the synthesis of nanoparticles with controlled size and structure. In this method, a $\mathrm{Np}-\mathrm{KCl}$ composite is formed in a onepot reduction in THF at room temperature. $\mathrm{KCl}$ is an insoluble by-product of the reaction and serves as a matrix that traps the nanoparticles to avoid particle agglomeration and to control the coalescence of nanoparticles during thermal annealing up to $600{ }^{\circ} \mathrm{C}$. By varying the molar ratio of metal precursors and $\mathrm{KCl}$, as well as the time and temperature of annealing, the final particle sizes and crystalline order can be independently controlled. After thermal processing, nanoparticles can be released from the $\mathrm{KCl}$ matrix in an ethylene glycol-water solution and transferred to support materials forming a uniform Np-support composite. An in-situ STEM (scanning transmission electron microscope) study revealed that the mechanism of nanoparticle growth during annealing is dominated by particle-particle coalescence, although Ostwald ripening also occurs at a slower rate. A detailed study of the synthesis of ordered intermetallic $\mathrm{Pt}_{3} \mathrm{Fe}$ and $\mathrm{PtFe}$ nanoparticles with an average diameter of $4 \mathrm{~nm}$ is provided as two examples of the method. The generality of the method was extended to synthesis of other bi- and tri-metallic nanoparticles of platinum transition metal compounds. We expect that a wide variety of non-Pt metal 
containing nanoparticles can be also prepared using this same approach. 


\section{BIOGRAPHICAL SKETCH}

Hao Chen was born September $2^{\text {nd }}, 1981$ in Tianjin, China, where he grew up and spent most of his time before graduate school. After graduating from the high school, he enrolled at Nankai University for a B.S. degree in chemistry. Nankai University is the hometown of the author and known for organic synthesis throughout China. So naturally he developed a great interest in organic chemistry. Therefore, after graduation he began graduate education in surface chemistry and organic synthesis through a joint program at the Illinois Institute of Technology and Argonne National Laboratory in Chicago. After three years of study he received his MS and moved to the Chemistry Department at Cornell University, where he joined the DiSalvo group and began his PhD studies in material chemistry. At Cornell University, his primary interest was in the field of energy materials, especially fuel cell catalysts synthesis and cathode materials for lithium sulfur batteries. As the major achievement, he developed a synthetic and processing method to produce Pt based nanoparticles with controlled size, crystal structure and composition using the inorganic salt potassium chloride as a matrix to protect individual particles from agglomeration. 


\section{ACKNOWLEDGMENTS}

Five years of graduate study at Cornell University has been a precious experience for me. Thanks for my peers, lab mates, and friends because you are the ones who made my journey at Cornell both possible and enjoyable. First among these people is my advisor Frank DiSalvo. I am just so lucky to work with such a knowledgeable, enjoyable and generous person both as a scientist and a friend. His vision, attitude, wisdom and charming personality all make him a great person and a model for my life. I guess this is what we called a mentor, who will shape you to a better person before a better scientist. I also thank my other committee members, Prof. Abruña and Prof. Robinson for their availability so I could seek advice on electrochemistry and nanoparticle synthesis.

For the work described in this thesis "A Surfactant-Free Strategy for Synthesizing and Processing Intermetallic Platinum-Based Nanoparticle Catalysts", I received continuous help and support from the current and former members of the DiSalvo group, especially Brian Leonard, Deli Wang, Minh Nguyen, Doug DeSario and Kathryn Newton, an undergraduate who graduated with her BS in May 2013. I also thank Yingchao Yu, Houlin Xin John Grazul, and Mick Thomas for their help on electron microscopy. I thank Weidong Zhou for the collaborate work on the battery project. Thank you all for your help. I have also to thank my friends; there just too many ups and downs during this journey but your support were always there. There is no way for me to finish without you. However, there are too many names in my mind 
right now and I cannot put all here. So I decide to list just a few who have accompanied me the most during this journey: Keenan Valentine, Weidong Zhou, Chenyue Wang, Deli Wang, Peng Chen and Minh Nguyen. Thank you!

I also thank for my parents for their support since day 1 of my life. It is your support makes my heart strong and persistent on my goals. Your love has always been so warm and powerful, that pushes me forward when I met difficulties. Thank you!

I also greatly appreciate the support of the Department of Energy, Basic Energy Sciences division through grant number DE-FG02-87ER45298. This grant enabled all the work I have undertaken here at Cornell, paying my tuition, purchasing materials and supplies, paying facilities charges and, of particular help to me personally, my stipend. This support ultimately comes from the American taxpayer, to whom I am deeply grateful. 


\section{TABLE OF CONTENTS}

Biographical Sketch iii

Acknowledgement

Chapter 1 INTRODUCTION 1

1.1 Fuel cells 1

1.2 Alloys and intermetallics as catalysts for ORRs 3

1.3 The synthesis of intermetallic nanoparticles 9

$\begin{array}{ll}\text { References } & 11\end{array}$

Chapter 2 INTERMETALLIC NANOPARTICLE SYNTHESIS 15

2.1 The Liquid Reduction method 15

$\begin{array}{ll}\text { 2.1.1 The reducing agent } & 15\end{array}$

$\begin{array}{ll}\text { 2.1.2 The Solvent } & 18\end{array}$

$\begin{array}{lr}\text { 2.1.3 The metal precursor } & 18\end{array}$

2.2 $\mathrm{LiEt}_{3} \mathrm{BH}$ and $\mathrm{KEt}_{3} \mathrm{BH}$ as reducing agent 22

2.2.1 Using $\mathrm{LiEt}_{3} \mathrm{BH}$ and $\mathrm{KEt}_{3} \mathrm{BH}$ as reducing agent 22

2.2.2 The thermal annealing process to achieve the intermetallic phase 25

2.3 Conclusion 31

References

Chapter 3 THE NANOPARTICLE-KCl (Np-KCl) METHOD PART I: THE $\begin{array}{ll}\text { SYNTHESIS } & 35\end{array}$

3.1 Introduction 35 
3.1.1 Synthetic criteria

3.1.2 The available methods

3.2.1 The idea of the $\mathrm{Np}-\mathrm{KCl}$ method 39

3.2.2 Forming the $\mathrm{Np}-\mathrm{KCl}$ assembly I: the synthesis $\quad 40$

3.2.3 Forming the $\mathrm{Np}-\mathrm{KCl}$ assembly II: the anions 48

3.2.4 Synthesis of the intermetallic phase with controlled sizes 53

3.3 The growth mechanism of bimetallic nanoparticles in the $\mathrm{KCl}$ matrix during $\begin{array}{ll}\text { thermal annealing } & 64\end{array}$

3.3.1 The method for studying the growth mechanism of nanoparticles in the $\mathrm{KCl}$ matrix during thermal annealing

3.3.2 The synthesis of $4 \mathrm{~nm}$ intermetallic PtFe nanoparticles using the $\mathrm{Np}-\mathrm{KCl}$ method 66

3.3.3 The particle growth mechanism during thermal annealing of the $\mathrm{Np}-\mathrm{KCl} 77$

3.4 To optimize the $\mathrm{Np}-\mathrm{KCl}$ method $\quad 90$

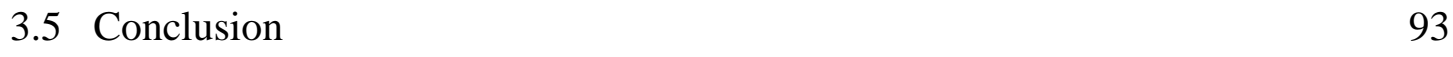

$\begin{array}{ll}\text { References } & 94\end{array}$

Chapter 4 THE NANOPARTICLE-KCl (Np-KCl) METHOD PART II:

RELEASING THE NANOPARTICLES FROM THE MATRIX 99

$\begin{array}{lll}4.1 & \text { Introduction } & 99\end{array}$

$\begin{array}{ll}\text { 4.2 The } \mathrm{BF}_{3} \text { method } & 100\end{array}$

$\begin{array}{ll}\text { 4.3 The Ethylene Glycol method } & 108\end{array}$ 
4.5 Conclusion 123

$\begin{array}{ll}\text { References } & 125\end{array}$

Chapter 5 THE NANOPARTICLE-KCl (Np-KCl) METHOD PART III: THE

$\begin{array}{ll}\text { GENERALITY } & 126\end{array}$

$\begin{array}{ll}5.1 \text { Introduction } & 126\end{array}$

$\begin{array}{ll}\text { 5.2 The metal hydride formation } & 127\end{array}$

5.3 The synthesis of $\mathrm{Pt}_{3} \mathrm{Mn}$ intermetallic nanoparticles 133

5.4 The synthesis of PtNi intermetallic nanoparticles 138

5.5 The synthesis of $\mathrm{Pt}_{2} \mathrm{CoFe}$ intermetallic nanoparticles 143

5.6 The synthesis of $\mathrm{Pt}_{3} \mathrm{~V}$ intermetallic nanoparticles 148

5.7 The synthesis of $\mathrm{Pt}_{3} \mathrm{Ti}$ intermetallic nanoparticles 152

5.8 Conclusion 156

$\begin{array}{ll}\text { References } & 157\end{array}$ 


\section{CHAPTER 1}

\section{INTRODUCTION}

\subsection{Fuel cells}

Due to the Carnot effect, in a traditional combustion engine, the automobile can only convert $25 \%$ of the energy from combustion into the mechanical motion of the vehicle. The rest is lost as heat. The idea of fuel cells is to divide the direct combustion of fuel and oxygen into two separate catalyst-assisted half reactions: the oxidation of fuel and the reduction of oxygen. In this process, electrons are generated from the fuel electrochemically, passed through an external load to do work, and finally used in the reduction of oxygen at the cathode. This type of electrochemical conversion has a very high theoretical energy conversion efficiency, up to $100 \%$ of the reaction Gibbs free energy. Current large-scale fuel cells (e.g. solid oxide or polymer membrane fuel cells) are somewhat better than $50 \%$ efficient (based on the free energy). But if material advances can be made, practical efficiencies of over $85 \%$ appear possible. In some cases efficiencies approaching $100 \%$ even seem attainable.

The polymer electrolyte membrane fuel cells (PEMFCs) are particularly attractive for operation near room temperature, and are expected to have a broad range of applications in transportation and portable power markets. This thesis will focus on the catalysts for PEMFCs, especially the synthetic method to prepare novel cathode catalysts. Before we go into details, let's go through different components of PEMFCs 
and their functionalities.

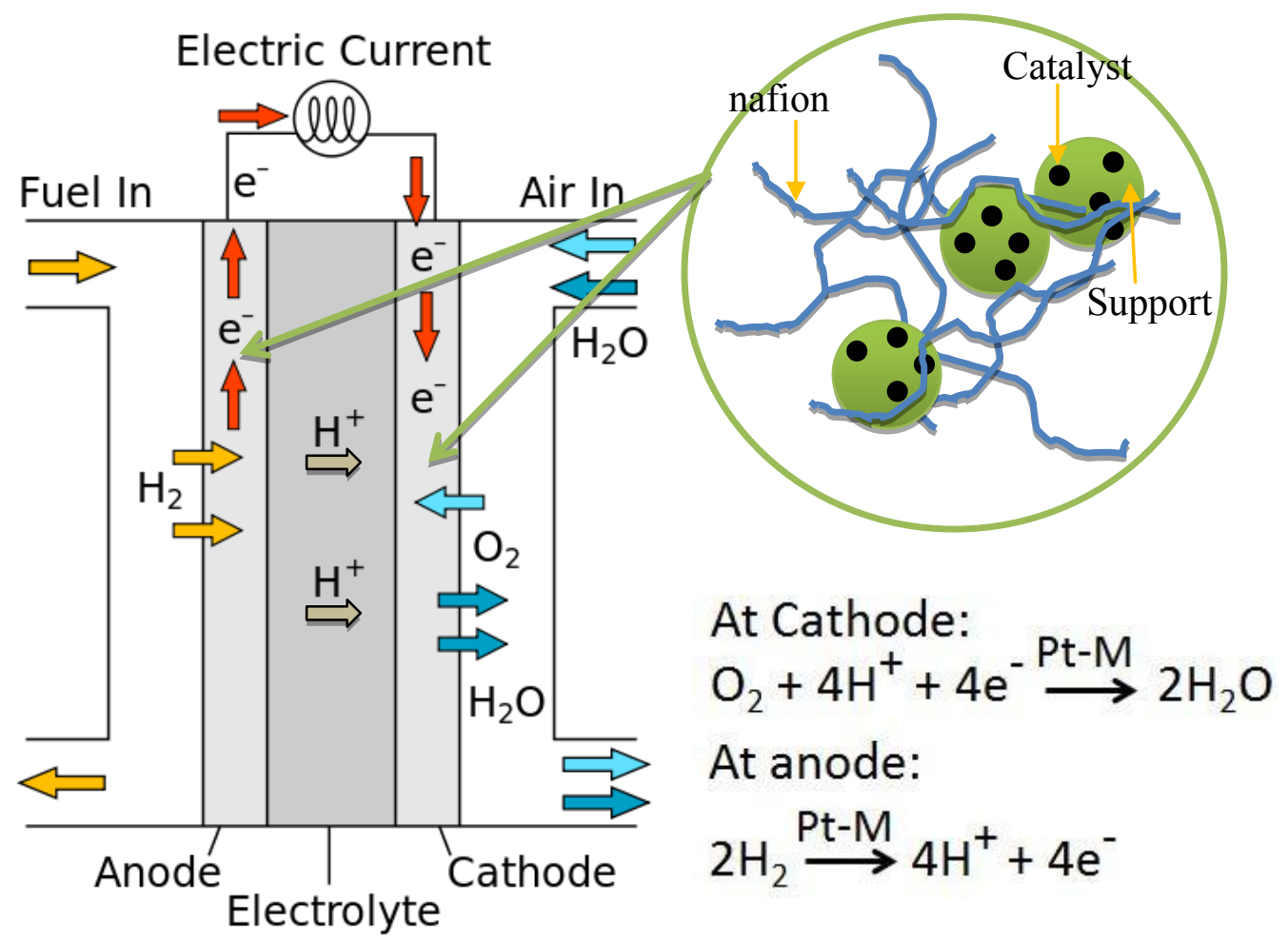

Figure 1.1: A simplified schematic diagram of a PEMFC (polymer electrolyte membrane fuel cell)

Shown in figure 1.1 is a cartoon of a PEMFC. At the anode the fuel (in this case, hydrogen gas) is oxidized to release electrons. At present, only a few substances can used as fuels in the PEMFCs besides hydrogen; these are single carbon based small molecules such as, especially methanol and formic acid. In all cases the fuels are oxidized at the anode releasing $\mathrm{H}^{+}$and $\mathrm{CO}_{2}$, if carbon is present, as well as the valence electrons released from the oxidation reaction. Likewise, at the cathode oxygen molecules are reduced by the electrons that flow through the electrical load and water 
is formed. This latter step is called the oxygen reduction reaction (ORR). Both the fuel oxidation and the ORR are normally catalyzed by platinum $(\mathrm{Pt})$ based materials.

However, compared to the fuel oxidation reaction, the ORR at the cathode has always been the most critical challenge to overcome in terms of the energy conversion efficiency due to the slow kinetics of the ORR. Therefore, the focus of this thesis will be on the synthesis of advance catalyst materials for the cathode. Pt is still one of the better catalysts available. However, the slow kenetics of reduction leads to large overpotentials (efficiency loss) when operating at reasonable current densities. By alloying Pt with other metals, researchers hope to both improve the activity and improve the durability of the catalysts.

\subsection{Alloys and intermetallics as catalysts for the ORR}

Currently Pt is one of the highest activity ORR catalysts in PEMFCs. Recent studies showed alloying Pt with transition metals does not only save the precious Pt but also alters the electronic and atomic structure of the catalytic surface.

For example, for the Pt-M (M = later transition metals) nanoparticle catalysts, after running a few cycles under the acidic electrolyte, the secondary metal near the surface will eventually dissolve into the electrolyte leaving Pt enriched surface layers ${ }^{1}$. The rate of the ORR on the alloys of $\mathrm{Pt}$ and $3 \mathrm{~d}$ metals shows a strong correlation with the nature of the alloying component (figure 1.2) ${ }^{2,3}$. According to recent studies, the alloys of $\mathrm{Pt}$ with $\mathrm{Ni}, \mathrm{Co}, \mathrm{Fe}, \mathrm{Cu}$, etc. show a 2 to 3 fold improvement in ORR activity 
compared to pure Pt. The enhancement of the activity from the alloying effect shows a volcano-shaped variation according to experimental studies ${ }^{2}$.

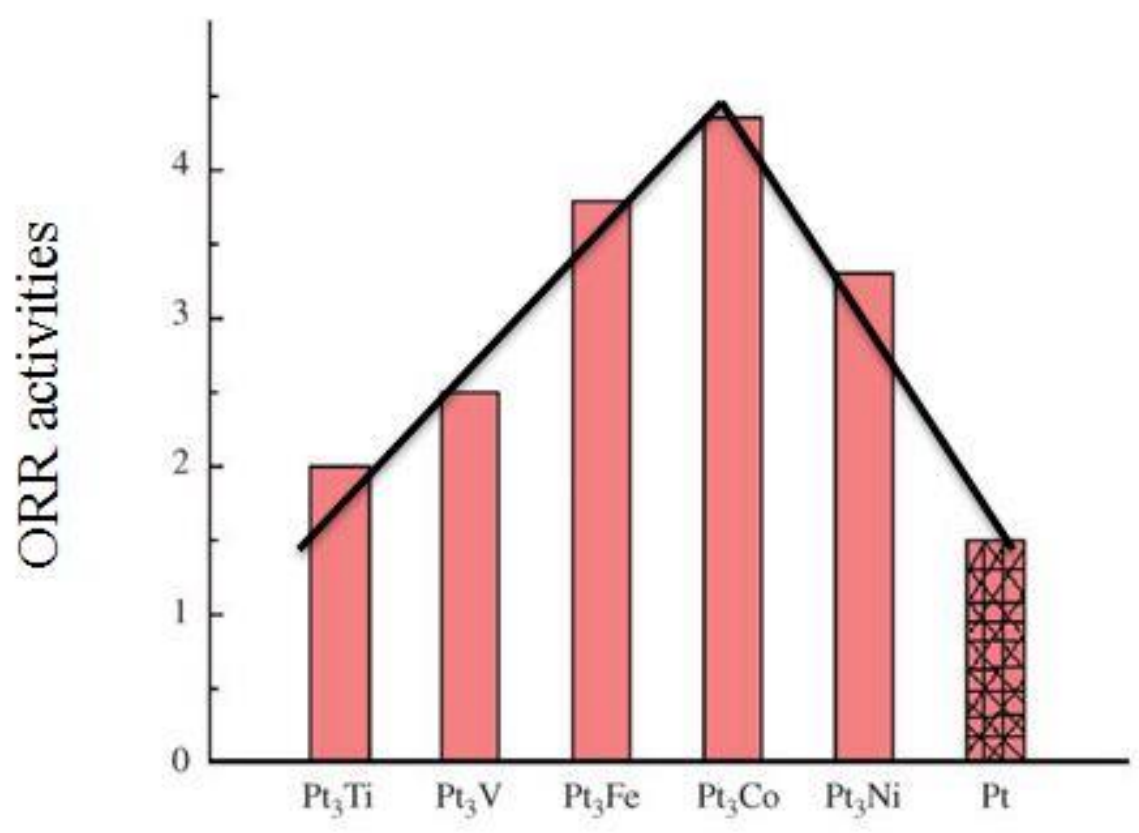

Figure 1.2: Specific activity of Pt and Pt3M electrodes expressed as a kinetic current density for the ORR at $0.9 \mathrm{~V}$ vs. $\mathrm{RHE}$ (in $0.1 \mathrm{~m} \mathrm{HClO}_{4}$ at $333 \mathrm{~K}$. RHE $=$ reversible hydrogen electrode). Reaction conditions: a Pt-M disc electrode in $0.1 \mathrm{~m} \mathrm{HClO}_{4}$ in an electrochemical cell swept at $50 \mathrm{mV} / \mathrm{sat} 333 \mathrm{~K}(1600 \mathrm{rpm}$;). The image is modified from the work of Stramenkovic et $\mathrm{al}^{2}$.

Such volcano-shaped effects are thought to originate from small changes in the electronic structure and/or the Pt-Pt bond lengths of the Pt enriched surface relative to 
pure Pt. Depending on the atomic arrangement of the surface and bulk atoms, The lattice mismatch between the surface (Pt rich) and the bulk (alloy structure) may generate compressed or expanded arrangements of the surface atoms (surface strain $)^{4,5}$. As such, by altering the alloying transition metals, the strain on the Pt skin changes. As a result, the $\mathrm{d}$ band energy of the Pt skin is altered with the alloying transition metals. In other words, by changing the alloying elements, the interaction strength between intermediates of the ORR (adsorbed $\mathrm{O}_{2}$ and $\mathrm{OH}$ surface species) and the Pt skin can be modified. Such subtle variations eventually determine the kinetics of the ORR. On this basis, to explain this volcano-shaped effect, a kinetic model was developed by Norskov et $\mathrm{al}^{6}$, where the rate of the ORR at a given potential is correlated with a function of a single parameter — the oxygen chemisorption energy on the catalyst surface, denoted as DEO. Figure 1.3 shows how this model explains the volcano-shaped dependence of the rate on DEO.

Shown in figure 1.4 is a cartoon of a typical ORR via a 4 electron pathway. For metals that bind oxygen too strongly (to the left of the maximum, figure 1.3), the ORR rate is limited by the rate of removal of the adsorbed $\mathrm{O}$ and $\mathrm{OH}$ species; that is, the surface is oxidized and thus unreactive, Such a mechanism is also suggested by Markovic et $\mathrm{al}^{7,8}$. For metal surfaces that bind oxygen too weakly, the rate is limited by the transfer of electrons and protons to the adsorbed $\mathrm{O}_{2}$. As shown in figure 1.3, according to the model developed by Norskov et al, the free energies of all intermediates of the ORR can be calculated as a function of the cell potential. The ORR activity of $\mathrm{Pt}$ nanoparticles can thus be tuned by alloying it with different transition metals. 


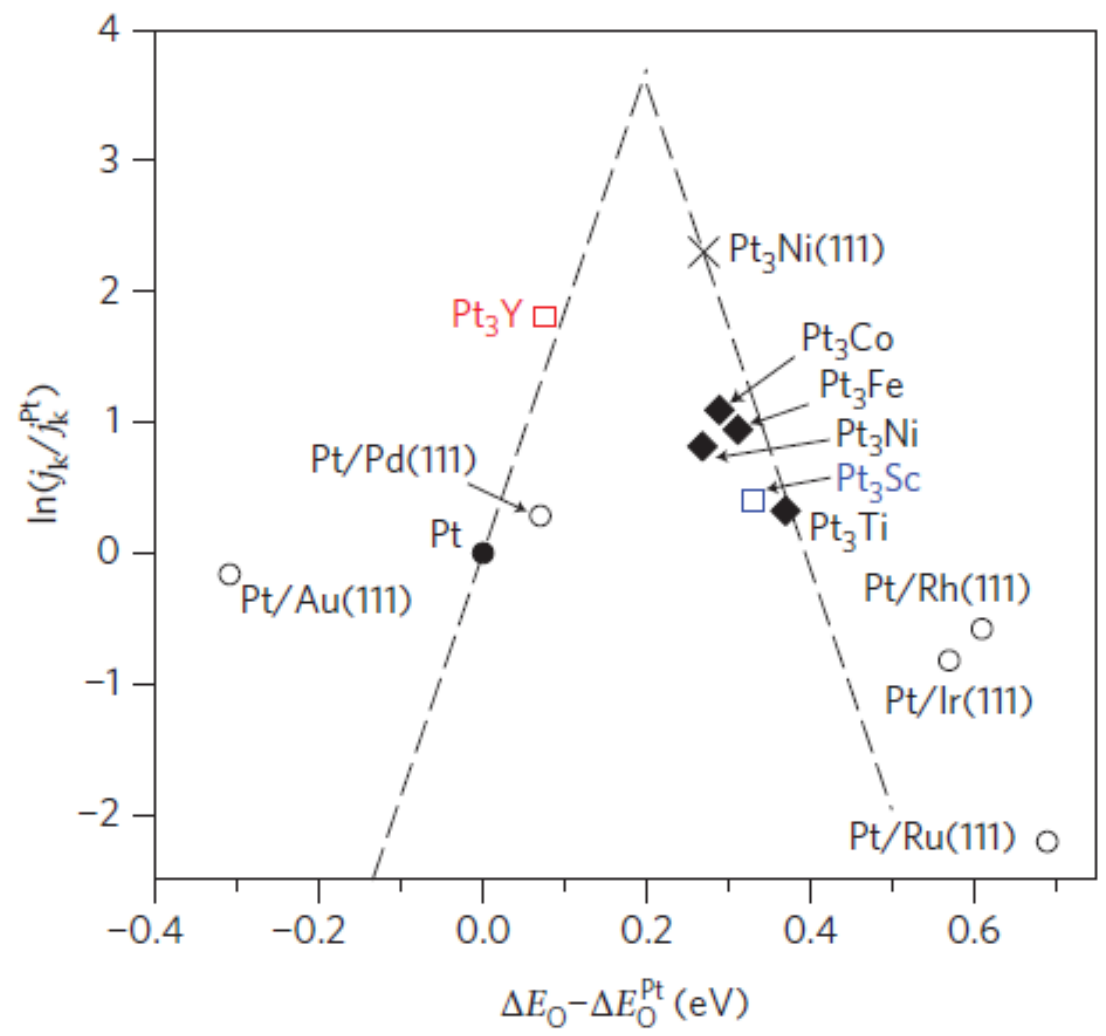

Figure 1.3: Volcano plots for the ORR on Pt-based transition metal alloys. Measured kinetic current density for a range of alloy electrocatalysts with Pt 'skins' as a function of the calculated oxygen adsorption energy, DEO from literature reports. ${ }^{7-9}$ The dashed lines are from the DFT predictions. ${ }^{6,10}$ 


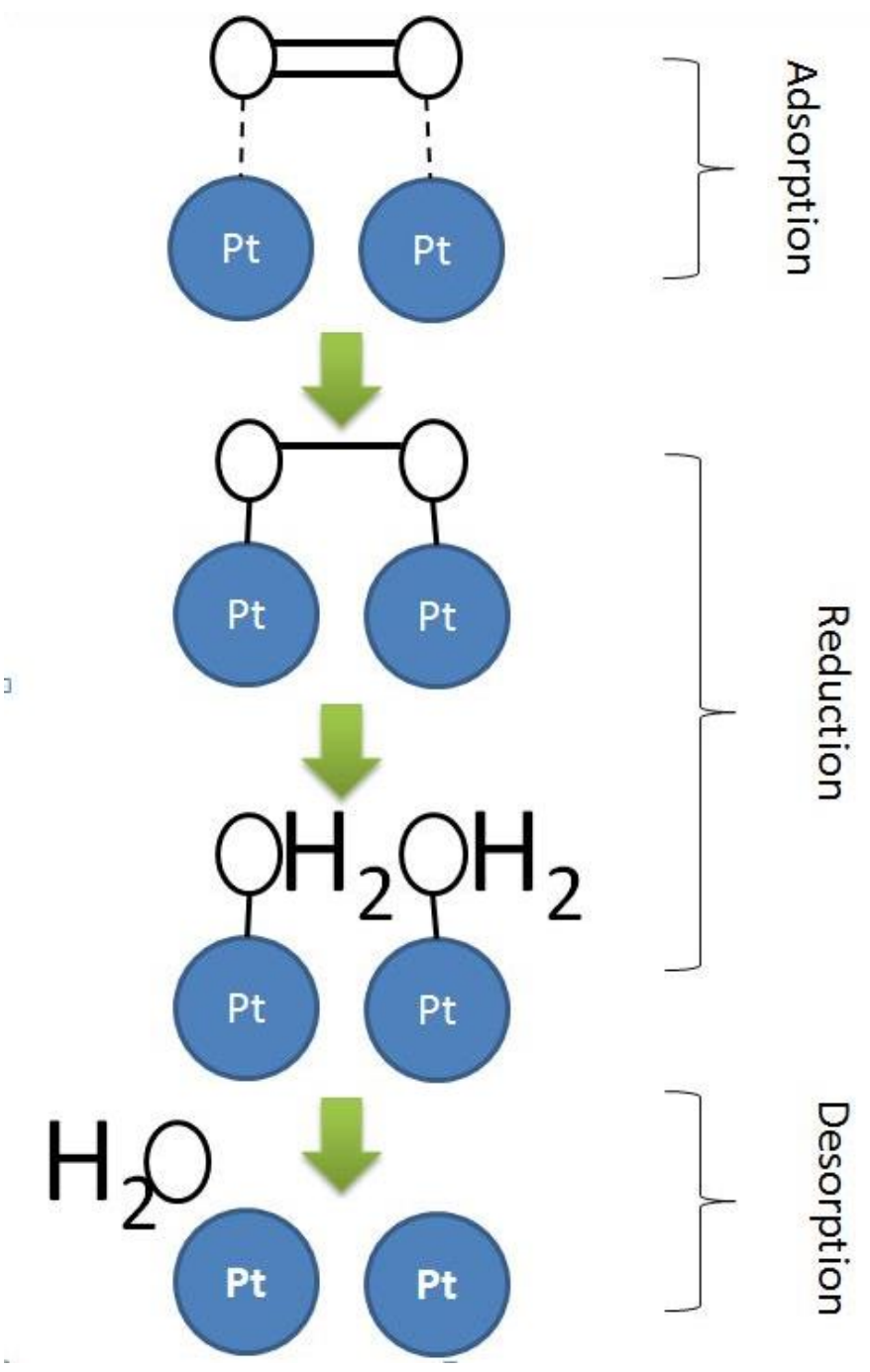

Figure 1.4: A typical ORR mechanism via a 4 electron pathway. To emphasize the oxygen adsorption and desorption process, the electron pathways are not marked in the image.

In a disordered alloy phase, each atomic site in the adopted crystal structure is randomly occupied by $\mathrm{Pt}$ and $\mathrm{M}$ with a probability that is determined only by the stoichiometric ratio of the two metals. However, in an ordered intermetallic compound of $\mathrm{Pt}_{\mathrm{n}} \mathrm{M}_{\mathrm{m}}$ ( $\mathrm{n}$ and $\mathrm{m}$ are integers) all atoms fully occupy distinct atomic sites (more 
formally different Wycoff positions), leading to an ordered structure, as in figure 1.5.

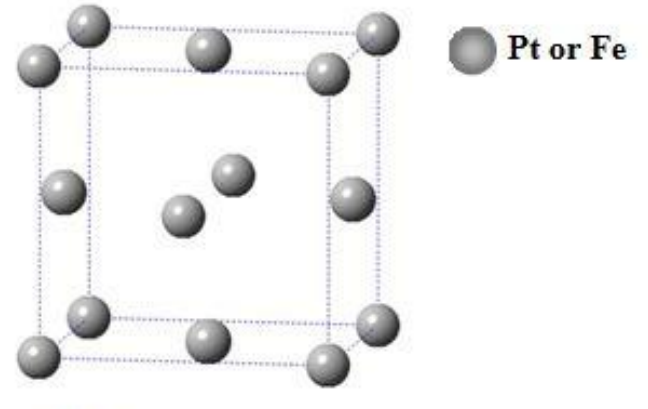

PtFe alloy:

Disordered structure

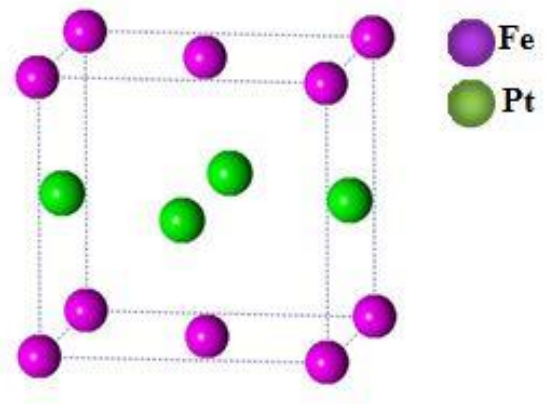

PtFe intermetallic:

Atomically ordered

Figure 1.5: Schematic illustration of the structure of a disordered FCC PtFe alloy (in this case $50 \%$ probability that any site is occupied by $\mathrm{Pt}$ or $\mathrm{Fe}$ ) and an ordered Tetragonal PtFe intermetallic structure.

Recent studies showed that the intermetallic structure does not only offer a better ORR activity but also has superior durability compared to its disordered alloy phase, at least for the $\mathrm{Pt}_{3} \mathrm{Co}^{11}, \mathrm{PtFe}^{12}$ and $\mathrm{PtFeAu}{ }^{13}$ systems. As for other systems, fewer reports have been shown regarding the ORR catalytic activities. In terms of the synthesis, there are three primary concerns for the intermetallic Pt-M nanoparticle catalysts, namely: forming the structurally ordered intermetallic phase; having the optimum particle 
sizes; and having a "clean" surface for catalytic reactions. However, to achieve the intermetallic structure a thermal annealing process is usually unavoidable for most of the Pt-M systems. For example, an annealing temperature for catalysts prepared near room temperature of about $500{ }^{\circ} \mathrm{C}$ or higher is needed to develop the order for $\mathrm{M}=$ first row transition metals. This temperature introduces tremendous challenges in keeping intermallic particle sizes small (near $5 \mathrm{~nm}$ ) and in preparing "clean" surfaces, while using published synthetic methods.

\subsection{The synthesis of intermetallic nanoparticles}

For several decades the synthesis of Pt based bimetallic nanoparticles has relied on using different long chain surfactant molecules that are strongly bound to the nanoparticle surface. These surfactants are used to control nanoparticle size as well as to prevent particle aggregation. There are still two fundamental issues in terms of the use of surfactants: 1) surfactants may block surface reaction sites, limiting their catalytic activity; 2) thermal annealing of the nanoparticles is often needed to develop atomic order or promote growth of certain facets. Under the latter conditions the surfactant may decompose leaving surface residues, such as carbon, that deactivate the catalyst. Even if clean surfaces are obtained, aggregation of the nanoparticles leads to significant decreases in active surface area per mass of catalyst. Some groups have reported the use of $\mathrm{MgO}^{12,14}$ or $\mathrm{SiO}_{2}$ coatings ${ }^{15-17}$ to prevent aggregation, but surfactants were still involved in key steps during the synthesis. Some solution phase chemical synthesis methods form nanoparticles on support materials without using any surfactant; ${ }^{18}$ however, such methods do not usually permit independent control of 
particle size, phase, composition, as well as nanoparticle loading on a catalyst support.

Another approach to achieve clean surfaces is based on using ethylene glycol as both the reducing agent and protecting ligand, which provides simultaneous control over particle size and composition..$^{19,20}$ In this case, ethylene glycol is a weak surfactant (low binding energy) and can be completely decomposed to gas phase products either electrochemically at high potentials or by heat treatment below $175{ }^{\circ} \mathrm{C}$, leaving relatively clean surfaces. Generally, this process is suitable for producing well dispersed nanoparticles of the more electronegative elements, such as $\mathrm{Au}, \mathrm{Pt}, \mathrm{Rh}$, $\mathrm{Ru}^{21,22}$ Alloys and intermetallic phases are difficult to prepare by this method, since the reduction rates by the ethylene glycol for different elements vary widely. In any case, ethylene glycol that binds to the metal surface is known to be fully decomposed to gaseous products under heat treatment, ${ }^{19,20}$ especially above $150{ }^{\circ} \mathrm{C}$. Thus annealing at higher temperatures to obtain an ordered intermetallic phase cannot be carried out without significant particle agglomeration. ${ }^{19}$

Besides the superior ORR activities, in terms of the fuel oxidation reactions, the ordered intermetallic phases of Pt nanoparticles, when compared to their disordered alloy phases, have also been demonstrated to be better catalysts for fuel cell applications owing to their enhanced stability and poison tolerance. ${ }^{23-27}$ To provide a large surface area, the synthesis targets small particle sizes. However, when the nanoparticle sizes are too small (smaller than $3 \mathrm{~nm}$ in the case of pure Pt), these particles go through dissolution and coarsening, rendering poor stability. ${ }^{28} \mathrm{~A}$ comprehensive theoretical study predicted that in the cathode environment of 
PEMFCs, the particle morphology and size of Pt nanoparticles larger than $4 \mathrm{~nm}$ are relatively stable. ${ }^{29}$ However, a key challenge has remained for the current surfactant free synthetic methodologies to achieve such small particle sizes during the inevitable thermal annealing process to form the preferred ordered intermetallic structure. ${ }^{30}$

I this thesis, we report a new and surfactant-free approach for the preparation and handling of nanoparticles with controlled size and phase by using an ionic salt as a protective matrix during thermal annealing to limit nanoparticle growth. Using the preparation of ordered intermetallic $\mathrm{Pt}_{3} \mathrm{Fe}$ and $\mathrm{PtFe}$ nanoparticles as an example, the Pt-Fe nanoparticles were formed and protected by an insoluble by-product, in this case $\mathrm{KCl}$, in a THF solution. Instead of separate synthesis and protection steps, the $\mathrm{Np}-\mathrm{KCl}$ matrix was formed by a rapid room temperature liquid phase reduction of mixed metal chloride salts in THF. The $\mathrm{KCl}$ matrix protects the nanoparticles from aggregating during the synthesis and subsequent thermal annealing up to $600{ }^{\circ} \mathrm{C}$. The nanoparticles can then be released from the $\mathrm{KCl}$ and uniformly bound to a catalyst support in an ethylene glycol solution under sonication. The details of the synthetic method as well as the generality will be demonstrated in the following chapters.

\section{REFERENCES}

(1) Ruban, A. V.; Skriver, H. L.; Norskov, J. K. Phys Rev B 1999, 59, 15990.

(2) Stamenkovic, V.; Mun, B. S.; Mayrhofer, K. J. J.; Ross, P. N.; 
Markovic, N. M.; Rossmeisl, J.; Greeley, J.; Norskov, J. K. Angew Chem Int Edit 2006, 45, 2897.

(3) Stamenkovic, V. R.; Mun, B. S.; Mayrhofer, K. J. J.; Ross, P. N.; Markovic, N. M. J Am Chem Soc 2006, 128, 8813.

(4) Mavrikakis, M.; Hammer, B.; Norskov, J. K. Phys Rev Lett 1998, 81, 2819.

(5) Strasser, P.; Koh, S.; Anniyev, T.; Greeley, J.; More, K.; Yu, C. F.; Liu, Z. C.; Kaya, S.; Nordlund, D.; Ogasawara, H.; Toney, M. F.; Nilsson, A. Nat Chem 2010, 2, 454.

(6) Norskov, J. K.; Rossmeis1, J.; Logadottir, A.; Lindqvist, L.; Kitchin, J. R.; Bligaard, T.; Jonsson, H. J Phys Chem B 2004, 108, 17886.

(7) Markovic, N. M.; Schmidt, T. J.; Stamenkovic, V.; Ross, P. N. Fuel Cells 2001, 1, 105.

(8) Markovic, N. M.; Gasteiger, H. A.; Philip, N. J Phys Chem-Us 1996, 100,6715 .

(9) Greeley, J.; Stephens, I. E. L.; Bondarenko, A. S.; Johansson, T. P.; Hansen, H. A.; Jaramillo, T. F.; Rossmeisl, J.; Chorkendorff, I.; Norskov, J. K. Nat Chem 2009, 1, 552.

(10) Rossmeisl, J.; Karlberg, G. S.; Jaramillo, T.; Norskov, J. K. Faraday Discuss 2008, 140, 337.

(11) Wang, D. L.; Xin, H. L. L.; Hovden, R.; Wang, H. S.; Yu, Y. C.; Muller, D. A.; DiSalvo, F. J.; Abruna, H. D. Nat Mater 2013, 12, 81.

(12) Kim, J.; Lee, Y.; Sun, S. H. J Am Chem Soc 2010, 132, 4996.

(13) Zhang, S.; Guo, S. J.; Zhu, H. Y.; Su, D.; Sun, S. H. J Am Chem Soc 
2012, $134,5060$.

(14) Kim, J. M.; Rong, C. B.; Liu, J. P.; Sun, S. H. Adv Mater 2009, 21, 906.

(15) Yamamoto, S.; Morimoto, Y.; Tamada, Y.; Takahashi, Y. K.; Hono, K.; Ono, T.; Takano, M. Chem Mater 2006, 18, 5385.

(16) Yamamoto, S.; Morimoto, Y.; Ono, T.; Takano, M. Appl Phys Lett $2005,87$.

(17) Tamada, Y.; Yamamoto, S.; Takano, M.; Nasu, S.; Ono, T. Appl Phys Lett 2007, 90.

(18) Wang, D. L.; Subban, C. V.; Wang, H. S.; Rus, E.; DiSalvo, F. J.; Abruna, H. D. J Am Chem Soc 2010, 132, 10218.

(19) Bock, C.; Paquet, C.; Couillard, M.; Botton, G. A.; MacDougall, B. R. J Am Chem Soc 2004, 126, 8028.

(20) Wang, Y.; Ren, J. W.; Deng, K.; Gui, L. L.; Tang, Y. Q. Chem Mater 2000, $12,1622$.

(21) Bonet, F.; Guery, C.; Guyomard, D.; Urbina, R. H.; Tekaia-Elhsissen, K.; Tarascon, J. M. Int J Inorg Mater 1999, 1, 47.

(22) Bonet, F.; Delmas, V.; Grugeon, S.; Urbina, R. H.; Silvert, P. Y.; Tekaia-Elhsissen, K. Nanostruct Mater 1999, 11, 1277.

(23) Zhang, S. G. S. Z. H. S. D. S., S. J Am Ceram Soc 2012, 134, 4.

(24) Casado-Rivera, E.; Volpe, D. J.; Alden, L.; Lind, C.; Downie, C.; Vazquez-Alvarez, T.; Angelo, A. C. D.; DiSalvo, F. J.; Abruna, H. D. J Am Chem Soc 2004, 126, 4043 .

(25) Abe, H.; Matsumoto, F.; Alden, L. R.; Warren, S. C.; Abruna, H. D.; 
DiSalvo, F. J. J Am Chem Soc 2008, 130, 5452.

(26) Roychowdhury, C.; Matsumoto, F.; Mutolo, P. F.; Abruna, H. D.; DiSalvo, F. J. Chem Mater 2005, 17, 5871.

(27) Ghosh, T.; Leonard, B. M.; Zhou, Q.; DiSalvo, F. J. Chem Mater 2010, 22, 2190.

(28) Wikander, K.; Ekstroem, H.; Palmqvist, A. E. C.; Lindbergh, G. Electrochim Acta 2007, 52, 6848.

(29) Holby, E. F.; Sheng, W. C.; Shao-Horn, Y.; Morgan, D. Energ Environ Sci 2009, 2, 865 .

(30) Leonard, B. M.; Zhou, Q.; Wu, D. M.; DiSalvo, F. J. Chem Mater 2011, 23, 2499. 


\section{CHAPTER 2}

\section{INTERMETALLIC NANOPARTICLE SYNTHESIS}

\subsection{The Solution Phase Reduction method}

\subsubsection{The reducing agent}

With a potential benefit of scalable catalysts production, the DiSalvo group is interested in solution based nanoparticle synthesis, in which metal precursors are dissolved in a solvent and followed by a rapid reduction through the injection of a suitable reducing agent. Since the first row transition metals $(\mathrm{M})$ have a wide range electron negativities and reduction potentials, to reduce the precursors of $\mathrm{Pt}$ and $\mathrm{M}$ in such a way that Pt-M alloys are formed without significant phase segregation, a strong reducing agent and a fast reduction process is needed. Some examples of different types of reducing agents that have been explored by the DiSalvo and other groups in the past include: (1) the polyol process ${ }^{1-7}$ : This reduction process is slow (hours to days) with very different rates of reduction for different metals. Phase separation is usually found in the products; i.e. two elements are not uniformly distributed in each particle, rather one metal (usually Pt) is reduced first, then the other. These particles mix together on precipitation, but remain as two phases unless annealed so that interparticle diffusion can allow mixing. However, during thermal annealing, the particle size becomes too large and the particles sinter ${ }^{8}$. This method also could not adaptable to the binding of the nanoparticles to catalyst supports, especially those with low thermal stability. (2) Hydrazine reduction: Similar as Polyol, a reducing agent like 
hydrazine often produces two phase products. (3) Borohydride as reducing agent: Borohydrides, such as $\mathrm{ABH}_{4}$, with $\mathrm{A}=\mathrm{Li}$, Na or $\mathrm{K}$ are soluble in polar solvents such as methanol. $\mathrm{ABH}_{4}$ reacts slowly with methanol until the solution becomes alkaline enough for $\mathrm{H}^{-}$to remain unreactive. We did explore the use of methanol or ethanol as a solvent and borohydride as the reducing agent. For some systems using this method nanoparticle synthesis was found to be very reproducible. For example, previous group members prepared nano-particle $\mathrm{PtPb}^{9}$ at room temperature. Both unsupported and supported $\mathrm{PtPb}$ nanoparticles are prepared using this method. The as prepared $\mathrm{PtPb}$ without the presence of supports is amorphous or about $1 \mathrm{~nm}$ in domain size. However, ordered crystalline particles with 8 to $10 \mathrm{~nm}$ domain size are obtained when the product is stirred in the reaction container for 12 to 24 hours. Apparently the particles nucleate and grow crystalline nanoparticles during particle-particle collisions in solution, since no strong surfactants are used to prevent such agglomeration. Using this method, we are still limited to using metals with reduction potentials of $-0.3 \mathrm{~V}$ or higher. The other drawback is that some metals, especially for Fe and some adjacent metals, according to previous research in the group, react with borohydride to borides. (4) Borohydride in THF / glymes: These solvents are generally stable under strongly reducing conditions. For example, sodium naphthalide or potassium sodide, both very strong reducing agents, are stable in very dry THF or glymes. Elements as electropositive as Ti and rare earths, as well as more electronegative elements such as $\mathrm{C}$, have been reduced to their elemental form using these very strong reducing agents. ${ }^{10,11}$

Following the pioneering work of Bonneman, we have exploited the use of alkali 
metal triethylborohydrides in $\mathrm{THF}^{12-15}$ to prepare a wide variety of Pt-M alloy and intermetallic nanoparticles.
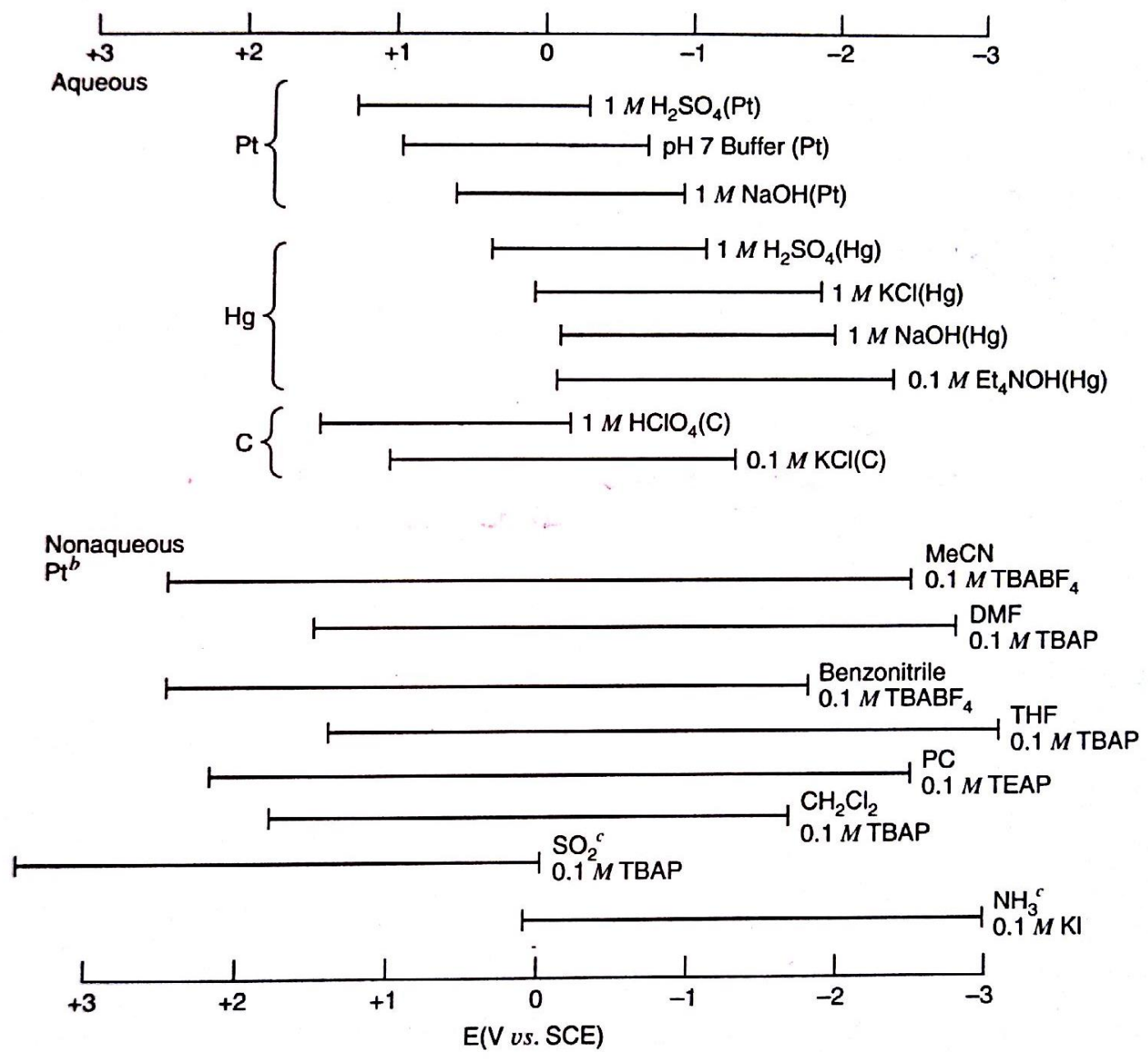

Figure 2.1: Estimated potential ranges for aqueous and nonaqueous solvents with Pt electrodes and selected supporting electrolytes. ${ }^{16,19,20}$ 


\subsubsection{The Solvent}

Using THF as the solvent has three major benefits. Firstly, as shown in figure 2.1, THF is stable at very reducing potentials or in the presence of strong reducing agents, as discussed above. ${ }^{16-18}$. Secondly, compared to diglyme, THF is less viscous and has a lower boiling temperature. Therefore, it is easier to be removed by vacuum distillation. Finally, a wide variety of appropriate metal precursors is soluble in THF.

\subsubsection{The metal precursor}

Since many metal acetylacetonates (M-acac) are usually soluble in ethereal solvents (e.g.: THF), they were initially selected as the metal precursors for the synthesis of PtM nanoparticles. In a typical synthesis, the metal acac precursors are dissolved in a THF solution under Ar, under vigorous stirring. The Pt-M nanoparticles are then formed by injection of the reducing agent solution (either triethylborohydride or $\mathrm{Na}$ naphthalene (Nap)). The precursor solution turns dark black instantaneously upon injection of the reducing agent, suggesting reasonably fast kinetics for the reduction. Using the synthesis of Pt nanoparticles as an example, the reaction is shown below:

$$
\begin{aligned}
& \mathrm{Pt}(\mathrm{acac})_{2}+2 \mathrm{LiEt}_{3} \mathrm{BH} \rightarrow \mathrm{Pt}+2 \mathrm{Li}(\mathrm{acac})+2 \mathrm{Et}_{3} \mathrm{~B}+\mathrm{H}_{2} \quad \text { (Equation 2.1) } \\
& \mathrm{Pt}(\mathrm{acac})_{2}+2 \mathrm{Na}(\mathrm{Nap}) \rightarrow \mathrm{Pt}+2 \mathrm{Na}(\mathrm{acac})+2 \mathrm{Nap} \quad \text { (Equation 2.2) }
\end{aligned}
$$

Without surfactants, the Pt particles quickly agglomerate and precipitate. Similarly, Pt- 
$\mathrm{M}$ can be prepared using this method by adding the appropriate $\mathrm{M}(\mathrm{acac})_{\mathrm{n}}$ precursor to the solution and the desired equivalents of reducing agent. The resulting powders were then washed using organic solvents like THF and hexanes, followed by vacuum drying. The products were then sealed in a silica tube under vacuum and annealed at different temperatures to achieve the intermetallic phase. We noticed, however, that the inside of the silica tubes had a black film deposited, likely from the decomposition of acac (figure 2.2a). Indeed, a thermogravometric analysis-mass spectrum (TGA-MS) study showed that the acac anion absorbs strongly with the as prepared nanoparticle, and decomposes upon annealing, thus covering the surface of the nanoparticle catalysts. Such side effect limits their electrochemical activity as well as the tube surface (figure 2.2b). The as-prepared Pt nanoparticles were heated to $550{ }^{\circ} \mathrm{C}$ with a ramping rate of $10{ }^{\circ} \mathrm{C}$ per minute under Ar in a Q50 thermal instrument. The outlet gas from the TGA was analyzed by a Hiden HPR20 MS system. As shown in figure 2.2b the $\mathrm{m} / \mathrm{z}$ with a value of 43 belongs to the fragment of acac molecule and the fragment with $\mathrm{m} / \mathrm{z}=128$ is from the naphthalene molecule. Clearly, the strong signal of $\mathrm{m} / \mathrm{z}=43$ suggests the acac anions as well as neutral Nap are still present after the nanoparticle synthesis, due to strong surface binding. More interestingly, the peak at around 1800 seconds, which corresponds to $330{ }^{\circ} \mathrm{C}$, suggests the interaction of acac and the surface of as- synthesized Pt nanoparticles is strong enough that the acac and naphthalene thermally decompose before beingreleased from the surface of Pt nanoparticles.

A detailed electrochemical study by former group member Brian Leonard also suggests that the acac residual hinders the catalytic activity of PtNi nanoparticle catalysts prepared using the metal acac precursors, independent of the reducing 
agent ${ }^{15}$. Many other attempts to prepare carbon free products from a variety of precursors that contained organic ligands or anions also failed. Thus we decided to work with totally inorganic precursors.

(a)
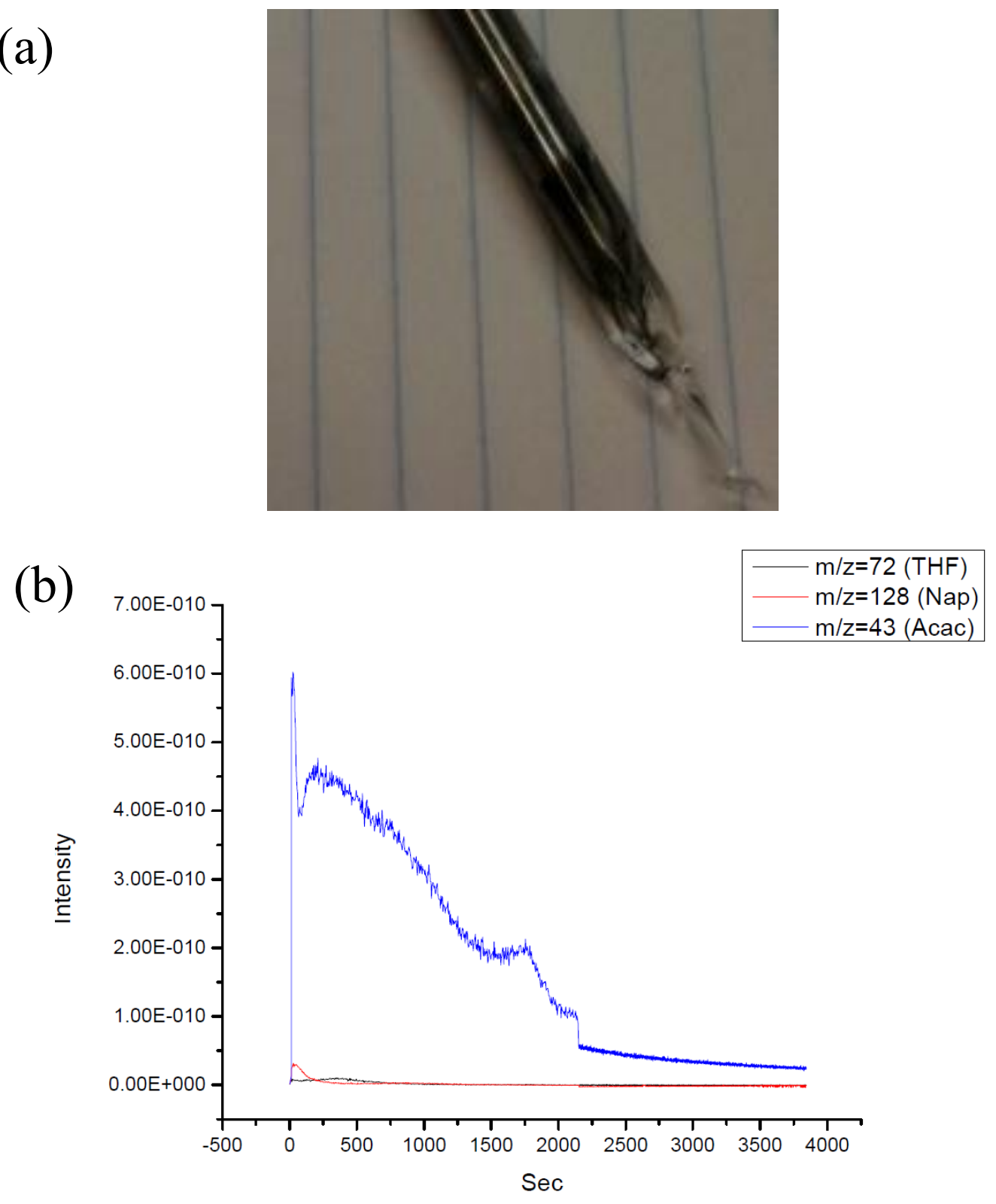

Figure 2.2: (a), The annealing tube and (b) the Thermogravity Analysis-Mass Spectrum (TGA-MS) study of the as-synthesized Pt nanoparticle using $\mathrm{Pt}(\mathrm{acac})_{2}$ as the precursor and Na naphthalide as the reducing agent. 
To eliminate the organics in the metal precursors, metal chloride, either as binary chlorides or as ternary lithium metal chloride salts (figure 2.3) can be used as a THF soluble source of metal cations. Using $\mathrm{LiEt}_{3} \mathrm{BH}$ and/or $\mathrm{KEt}{ }_{3} \mathrm{BH}$ as the reducing agent, metal chloride $\left(\mathrm{MCl}_{\mathrm{x}}\right)$ or ternary $\mathrm{Li}_{\mathrm{y}} \mathrm{MCl}_{\mathrm{x}}$ precursors of metals from groups 8, 9, 10, 11 in the periodic table and elements such as In, Sn, Sb, Bi can be reduced to form Pt$\mathrm{M}$ or even ternary compositions in a nanoparticle form. A detailed electrochemical study from Brian Leonard showed that the synthesized nanoparticle catalysts using metal chloride as precursors are much more active than catalysts of the same composition that were prepared from their metal organic counterparts (e.g. acac, cyclooctadiene $)^{15}$. Such results imply that the improvement of the activity is due to the cleaner surface of the products obtained from the chloride precursors.

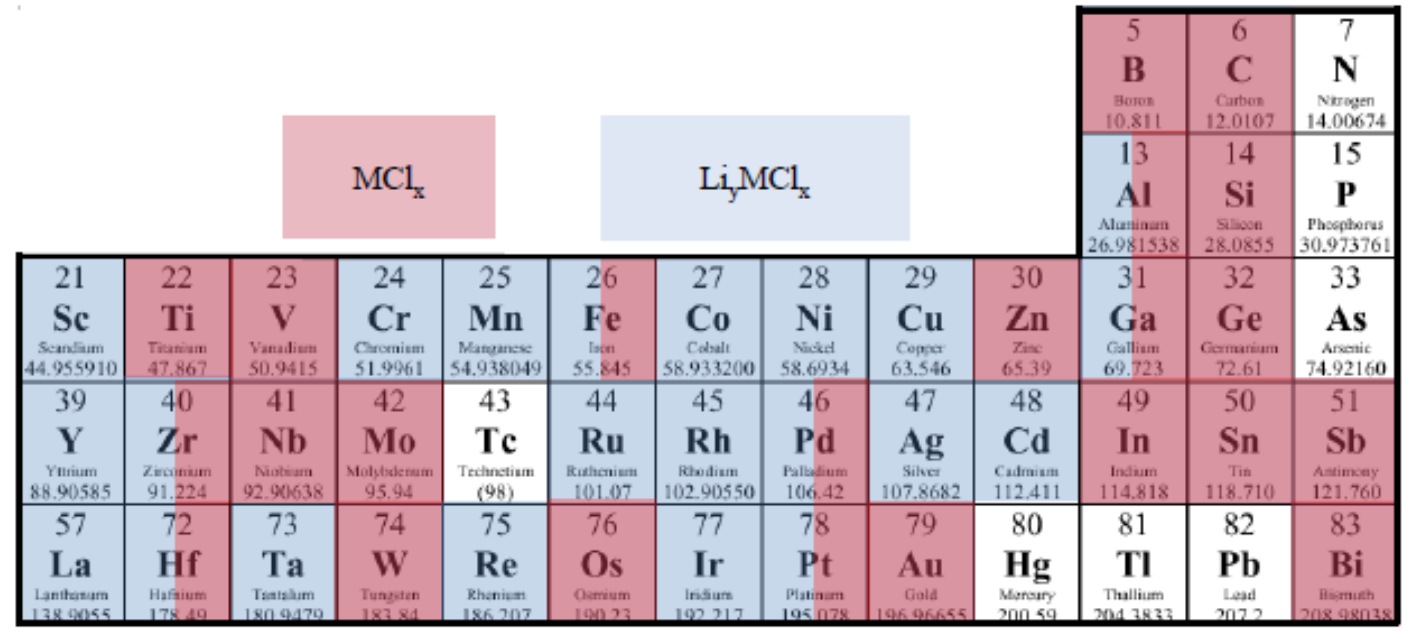

Figure 2.3: Virtually every metal in the periodic table can be put in THF solution as metal chloride (red) or ternary $\mathrm{Li}_{\mathrm{y}} \mathrm{MCl}_{\mathrm{x}}$ (blue) at useful concentrations (Exceptions: $\mathrm{Na}-\mathrm{Cs}, \mathrm{Mg}-\mathrm{Ba}, \mathrm{Hg}-\mathrm{Pb}$ ). When both $\mathrm{MCl}_{\mathrm{x}}$ and $\mathrm{Li}_{\mathrm{y}} \mathrm{MCl}_{\mathrm{x}}$ salts are available and soluble, the latter usually have higher solubility. 


\section{2 $\mathrm{LiEt}_{3} \mathrm{BH}$ and $\mathrm{KEt}_{3} \mathrm{BH}$ as reducing agent}

\subsubsection{Using $\mathrm{LiEt}_{3} \mathrm{BH}$ and $\mathrm{KEt}_{3} \mathrm{BH}$ as reducing agent}

As discussed above, using $\mathrm{LiEt}_{3} \mathrm{BH}$ and/or $\mathrm{KEt}_{3} \mathrm{BH}$ as the reducing agent and metal chlorides as precursors, a general synthetic method can be applied to synthesize Pt-M nanoparticle catalysts with metals from groups $8,9,10,11$ in the periodic table and elements such as $\mathrm{In}, \mathrm{Sn}, \mathrm{Sb}$ and $\mathrm{Bi}$. The reactions are carried out in an air and water free environment to prevent side reactions with the non-platinum metals.

Using the synthesis of PtFe nanoparticles as an example, the reduction processes using these two reducing agents are shown below:

$$
\begin{aligned}
& \mathrm{PtCl}_{4}+\mathrm{FeCl}_{3}+7 \mathrm{LiEt}_{3} \mathrm{BH} \rightarrow \mathrm{PtFe}+7 \mathrm{LiCl}+7 \mathrm{Et}_{3} \mathrm{~B}+3.5 \mathrm{H}_{2} \quad \text { (Equation 2.3) } \\
& \mathrm{PtCl}_{4}+\mathrm{FeCl}_{3}+7 \mathrm{KEt}_{3} \mathrm{BH} \rightarrow \mathrm{PtFe}+7 \mathrm{KCl}+7 \mathrm{Et}_{3} \mathrm{~B}+3.5 \mathrm{H}_{2} \quad \text { (Equation 2.4) }
\end{aligned}
$$

Again, in both cases, the solution immediately turned black on addition of the reducing agent. However, $\mathrm{KCl}$ is insoluble in THF, while $\mathrm{LiCl}$ has a solubility of 4.6 wt $\%$ and is dissociated into ions in in $\mathrm{THF}^{21}$. The chloride anion has some affinity for the metal nanoparticle surfaces and thus stabilizes the as-formed nanostructured PtFe colloids through columbic repulsion between the particles. As a result, the PtFe nanoparticles synthesized by $\mathrm{LiEt}_{3} \mathrm{BH}$ do not precipitate; rather they remain suspended in the THF solution. On the other hand, since the $\mathrm{KCl}$ is not soluble in THF solution, after reduction, the PtFe nanoparticles co-precipitate with the $\mathrm{KCl}$ particles to form a 
KCl-nanoparticle matrix. After reduction, the products were processed by different washing and drying steps. In the case of $\mathrm{LiEt}_{3} \mathrm{BH}$ reduction, the product can be precipitated by pumping off the solvent and/or by adding hexane to the THF solution. Once precipitated, they can be washed with $\mathrm{THF}$ to remove $\mathrm{LiCl}$ and dried to form agglomerated pure product. In the case of $\mathrm{KEt}_{3} \mathrm{BH}$ reduction micron sized composite particles precipitate readily.

TEM images of the as-prepared powders prepeared from reduction by the different alkyl borohydrides are shown in figure 2.4. In the transmission electron microscope (TEM) image of figure 2.4a, the black dots are the PtFe nanoparticles. Since the PtFe nanoparticles are the only powders left after work up, they are heavily agglomerated. In contrast, for the $\mathrm{KEt}_{3} \mathrm{BH}$ reduction, the $\mathrm{KCl}$ co-precipitates with the $\mathrm{PtFe}$ nanoparticles. More interestingly, as shown in figure $2.4 \mathrm{~b}$, the $\mathrm{KCl}$, which is gray in color, serves as a matrix for the PtFe nanoparticles. In the $\mathrm{KCl}$ matrix, the PtFe nanoparticles are isolated from each other, with roughly the same particle sizes as produced by $\mathrm{LiEt}_{3} \mathrm{BH}$ reduction. By analyzing broad areas of the samplest, the distributions of the PtFe nanoparticles on or in the $\mathrm{KCl}$ matrix are relatively uniform from region to region, which is also a general phenomenon for Pt alloys with other transition metals synthesized using this method. I therefore hypothesize that the nucleation and growth processes of both $\mathrm{Pt}-\mathrm{M}$ and $\mathrm{KCl}$ are occur nearly simultaneously or with similar rates. If that were not the case, we would expect that $\mathrm{KCl}$ and Pt-M products would phase separate on precipitation. A detailed discussion of the mechanism is presented in Chapter 3. 


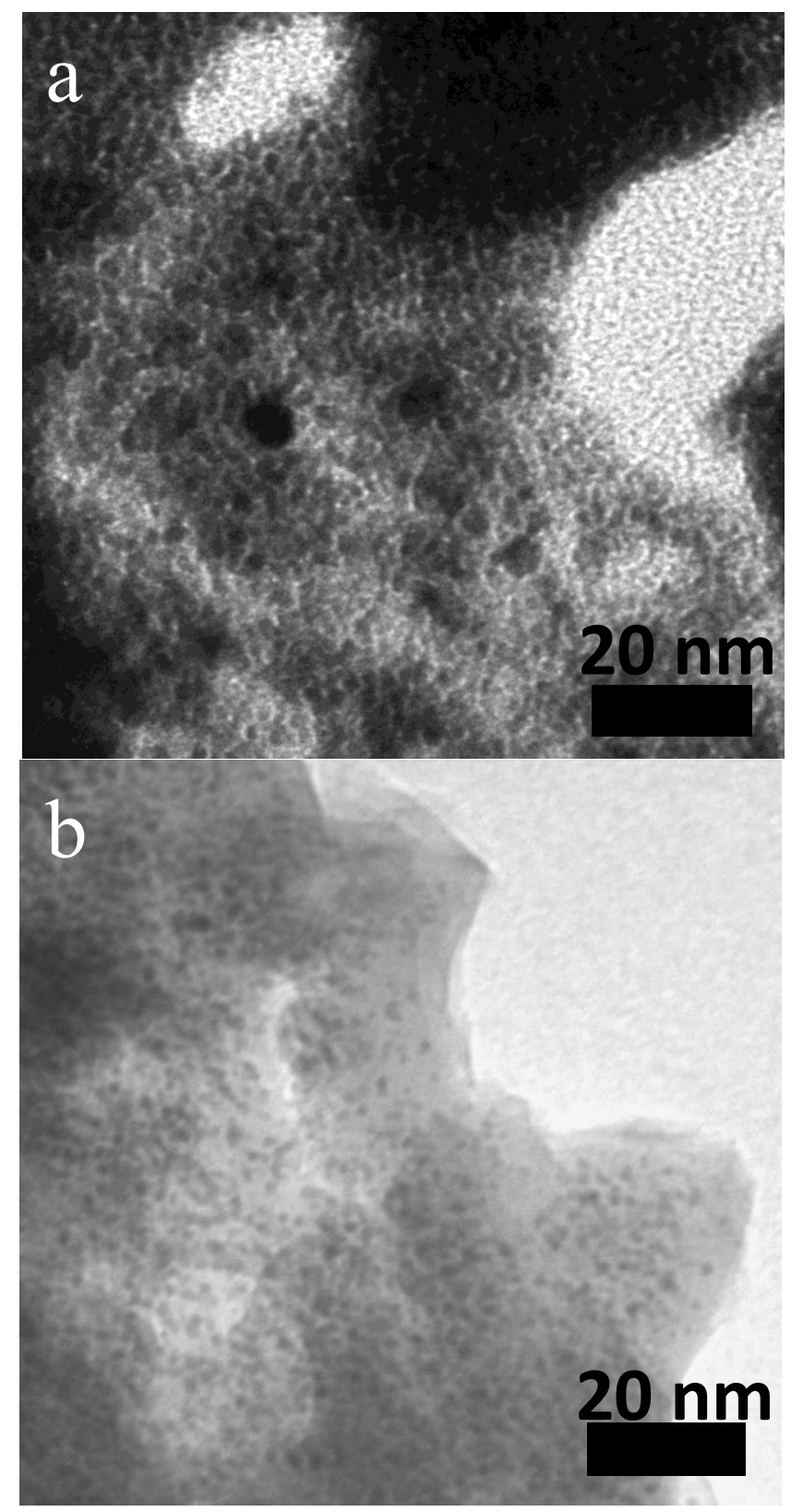

Figure 2.4: The transmission electron microscope (TEM) images of the as prepared products of the $\mathrm{LiEt}_{3} \mathrm{BH}$ reduction (a) and of the $\mathrm{KEt}_{3} \mathrm{BH}$ reduction (b). The black dots are the PtFe nanoparticles for (a) and (b). The gray area in (b) is the $\mathrm{KCl}$. 
2.2.2 The thermal annealing process to achieve the ordered intermetallic phase

As shown in figure 2.4, the as prepared nanoparticles are indeed very small (around 2 $\mathrm{nm}$ ). According to the powder X-ray Diffraction (XRD) (figure 2.5), they are either in

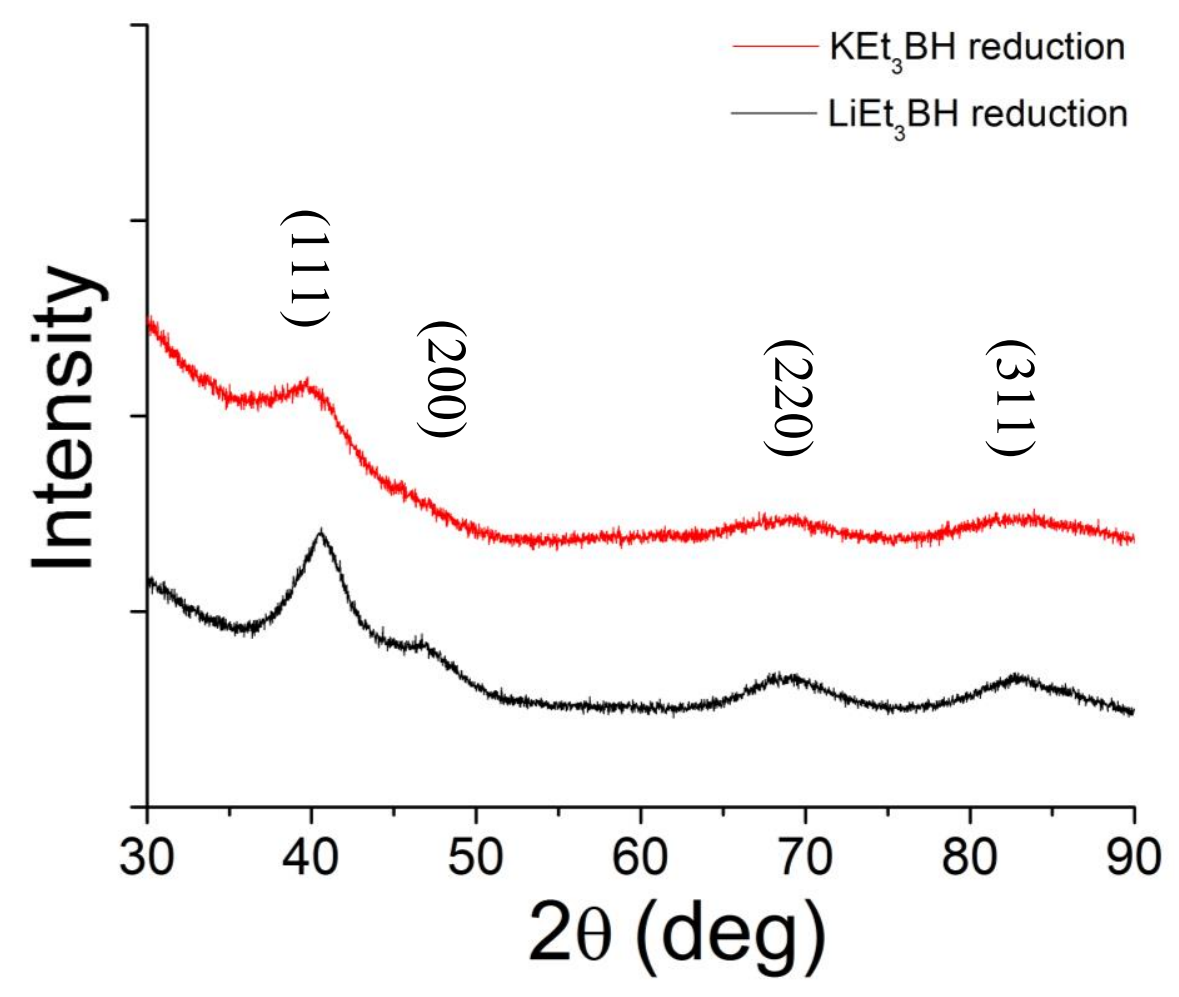

Figure 2.5: the XRD patterns of as-prepared PtFe nanoparticles through the reduction using $\mathrm{KEt}_{3} \mathrm{BH}$ (red) and $\mathrm{LiEt}_{3} \mathrm{BH}$ (black) as reducing agent. The metal precursors are $\mathrm{PtCl}_{4}$ and $\mathrm{FeCl}_{3}$ (The metal chlorides are always the precursors if not otherwise mentioned). 


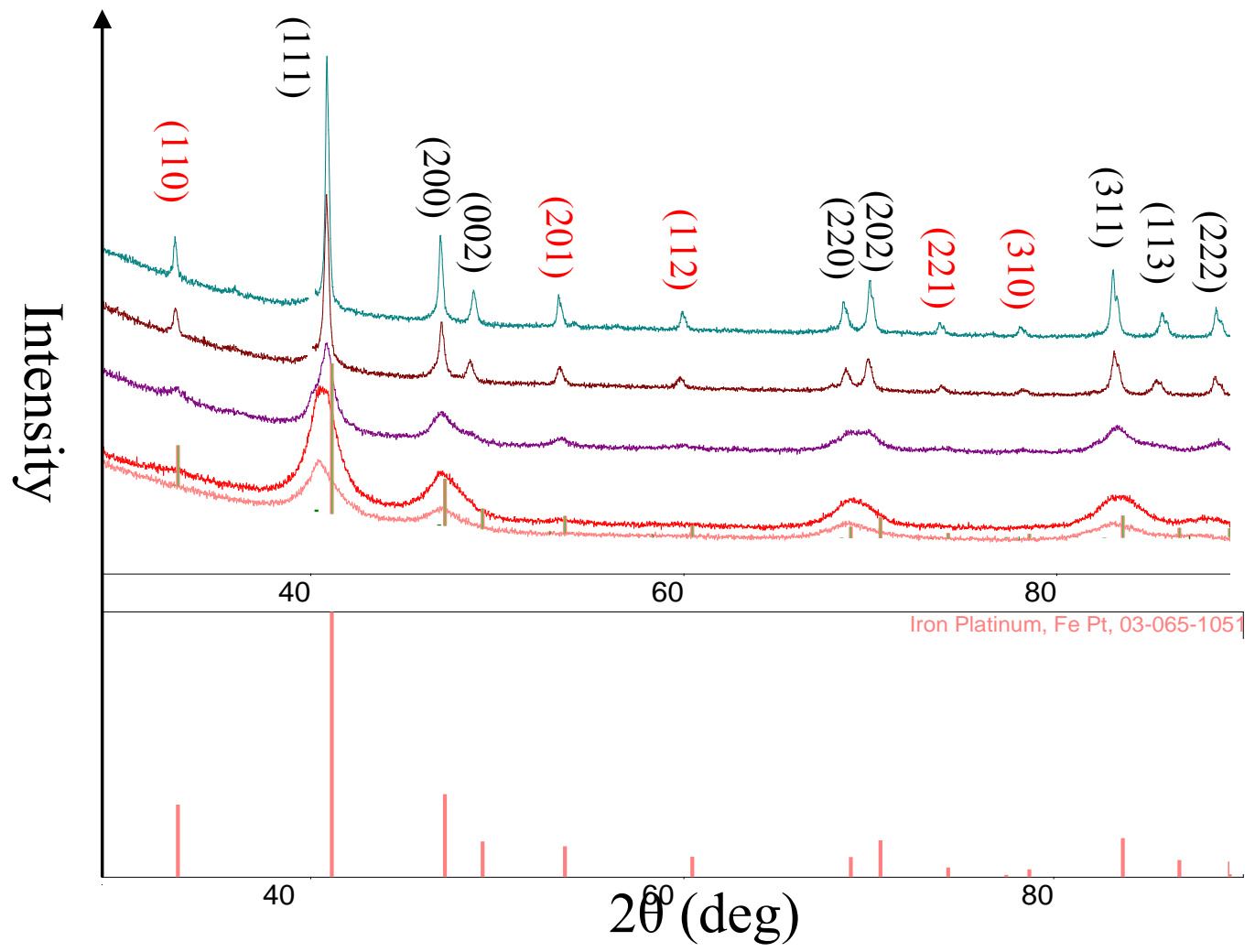

Figure 2.6: The XRD patterns of the annealed PtFe nanoparticles synthesized using $\mathrm{KEt}_{3} \mathrm{BH}$ as reducing agent. The samples are annealed for 12 hours under $400{ }^{\circ} \mathrm{C}$ (orange), $500{ }^{\circ} \mathrm{C}$ (red), $600{ }^{\circ} \mathrm{C}$ (blue), $700{ }^{\circ} \mathrm{C}$ (brown) and $800{ }^{\circ} \mathrm{C}$ (green). The XRDs were taken after removing the $\mathrm{KCl}$ salt. The lines on the bottom indicate the $\mathrm{XRD}$ peak positions of the intermetallic phase of PtFe (PDF \# 030651051). The indexes for the superlattice peaks from ordering are marked with red color. 
a face centered cubic (FCC) structure with really small domain sizes (smaller than 2 $\mathrm{nm}$ ) or in amorphous phases. Similar to the synthesis process mentioned previously, the resultant products shown in figure 2.4 were sealed in a silica tube under vacuum without air exposure. The whole synthesis, washing, drying and sealing process is water and air free, which is critical to prevent the oxidation of the secondary metal before thermal annealing to form the Pt-M intermetallic phases.

To convert the as prepared FCC PtFe into the ordered intermetallic structure, a thermal annealing process is required. For example, the as prepared powders from the $\mathrm{KEt}_{3} \mathrm{BH}$ reduction were sealed in a silica tube and annealed at $400{ }^{\circ} \mathrm{C}, 500{ }^{\circ} \mathrm{C}, 600{ }^{\circ} \mathrm{C}, 700{ }^{\circ} \mathrm{C}$ and $800{ }^{\circ} \mathrm{C}$ for 12 hours respectively. Shown in figure 2.6 are the XRD patterns of the annealed samples. After thermal annealing at $400{ }^{\circ} \mathrm{C}$ and $500{ }^{\circ} \mathrm{C}$ for 12 hours under vacuum, the PtFe nanoparticles remain in an alloy FCC structure. Since the peak widths (FWHM) only slightly decrease, the particle sizes have not changed significantly by this annealing step. However, after increasing the annealing temperature to $600{ }^{\circ} \mathrm{C}$ and higher, the ordered tetragonal phase is obtained. The observation of ordering peaks, such as the expected weak (110), (201), (112), (221) and (310) peaks demonstrates that the tetragonal ordered intermetallic phase is formed. In addition to the weak superlattice peaks, due to the ordering, the "a" axis and "c" axis of the unit cell are no longer equivalent. As a result, obvious peak splitting appears at around $2 \theta=47^{\circ}$ for (200) and (002), $2 \theta=69^{\circ}$ for (220) and (202), as well as $2 \theta=83^{\circ}$ for (311) and (113). Such results also confirmed that the ordered structure is formed. For the synthesis of $\mathrm{PtFe}$ using $\mathrm{LiEt}_{3} \mathrm{BH}$ as reducing agent, since there is no $\mathrm{KCl}$ as a matrix of the particles, the particles grow more rapidly and the 
ordering phase appears after thermal annealing at $400{ }^{\circ} \mathrm{C}$ for 12 hours. Such differences in the ordering temperature of agglomerated and matrix stabilized nanoparticles are also observed for other Pt-M systems ${ }^{15}$.

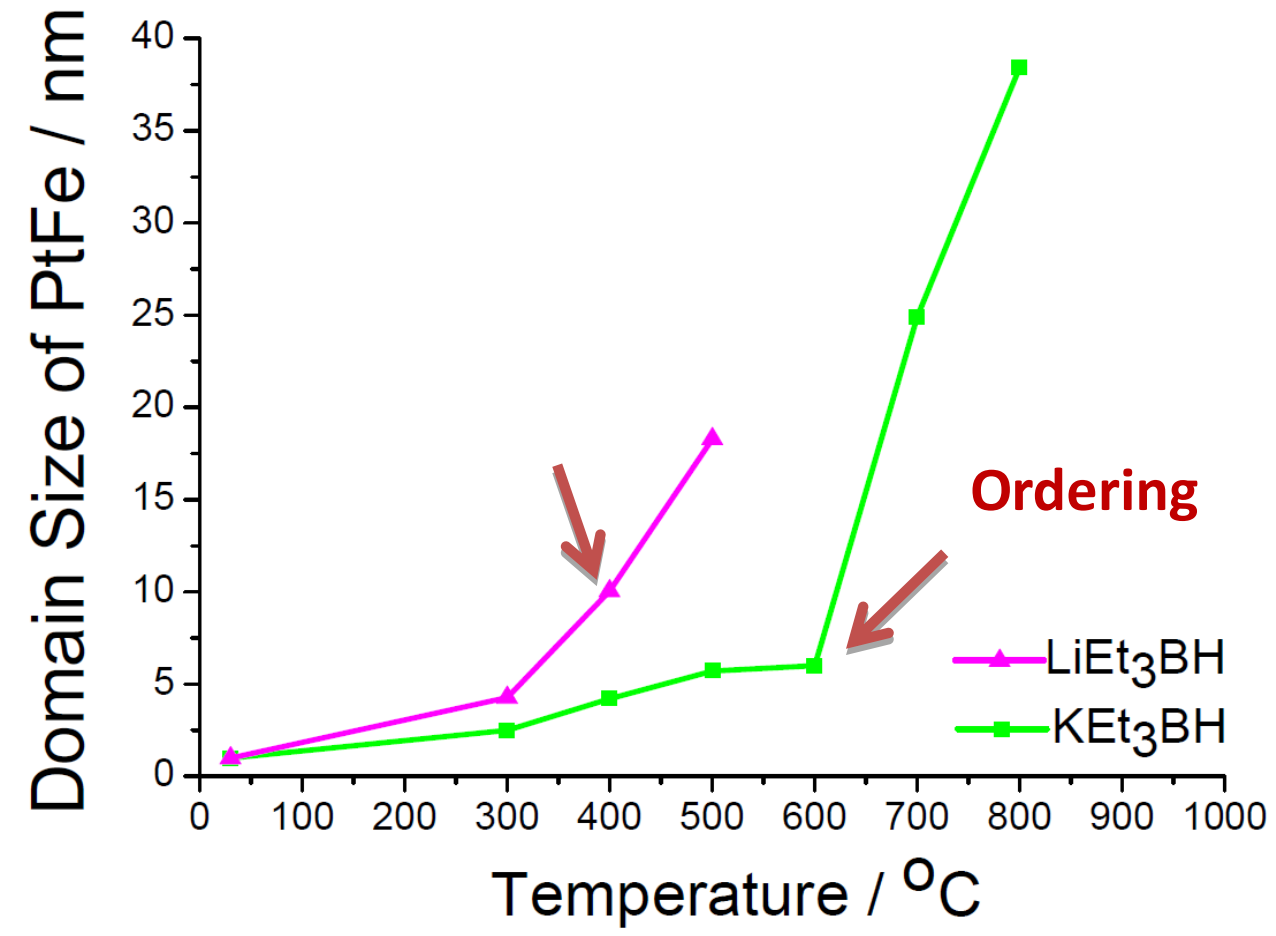

Figure 2.7: Domain sizes calculated from Scherrrer equation. The $\mathrm{LiEt}_{3} \mathrm{BH}$ reduction is marked by purple and the $\mathrm{KEt}_{3} \mathrm{BH}$ reduction is marked by green. The conditions where the ordered structures start to appear are marked by arrows. 
The domains sizes calculated from the XRD patterns of the annealed samples using Scherrer equation are shown in figure 2.7. ${ }^{22,23} \mathrm{The}_{\mathrm{LiEt}} \mathrm{BH}$ reduction products are shown in purple and the $\mathrm{KEt}_{3} \mathrm{BH}$ reduction products are shown in green. As shown in figure 2.7, at any given annealing condition the domains sizes of the resultant $\mathrm{PtFe}$ particles from $\mathrm{KEt}_{3} \mathrm{BH}$ reduction are always smaller than from the $\mathrm{LiEt}_{3} \mathrm{BH}$ reduction. This is expected since the $\mathrm{KCl}$ by-product from the $\mathrm{KEt}_{3} \mathrm{BH}$ reduction serves as a matrix (figure 2.4b) and inhibits the interparticle connection and diffusion of $\mathrm{Pt}$ and Fe, thus inhibiting particle growth and possibly the nucleation of the ordered phase. $\mathrm{KCl}$ has a melting temperature of $770{ }^{\circ} \mathrm{C}$, so as the annealing temperature reaches the melting temperature, the interparticle diffusion rate should increase dramatically. As shown in figure 2.7, for the $\mathrm{KEt}_{3} \mathrm{BH}$ reduction products, once the annealing temperature goes up to $700{ }^{\circ} \mathrm{C}$ and higher, there is a rapid growth of the domain sizes for $\mathrm{PtFe}$ nanoparticles. Such observations strongly suggest that the $\mathrm{KCl}$ matrix is playing an important role in suppressing the interparticle diffusion of Pt-M made from $\mathrm{KEt}_{3} \mathrm{BH}$ reduction.

To further understand the effects of the $\mathrm{KCl}$ matrix, the distribution of particle sizes from the annealed samples were also determined. We studied the products that showed ordering at the lowest annealing temperatures. They are shown in figure 2.8 with the scanning electron microscope (SEM) image for annealed $\mathrm{PtFe}$ made with $\mathrm{LiEt}_{3} \mathrm{BH}$ (figure 2.8a) and the TEM image of the annealed PeFe made with $\mathrm{KEt}_{3} \mathrm{BH}$ (figure 2.8b). Clearly, even though the domain size for these two samples are only 2 or $3 \mathrm{~nm}$ apart, the PtFe particles (which may contain multiple domains) from the $\mathrm{LiEt}_{3} \mathrm{BH}$ reduction are significantly larger than that from the $\mathrm{KEt}_{3} \mathrm{BH}$ reduction. Along with the 
domain sizes analysis, this result also confirms that the $\mathrm{KCl}$ matrix inhibits the

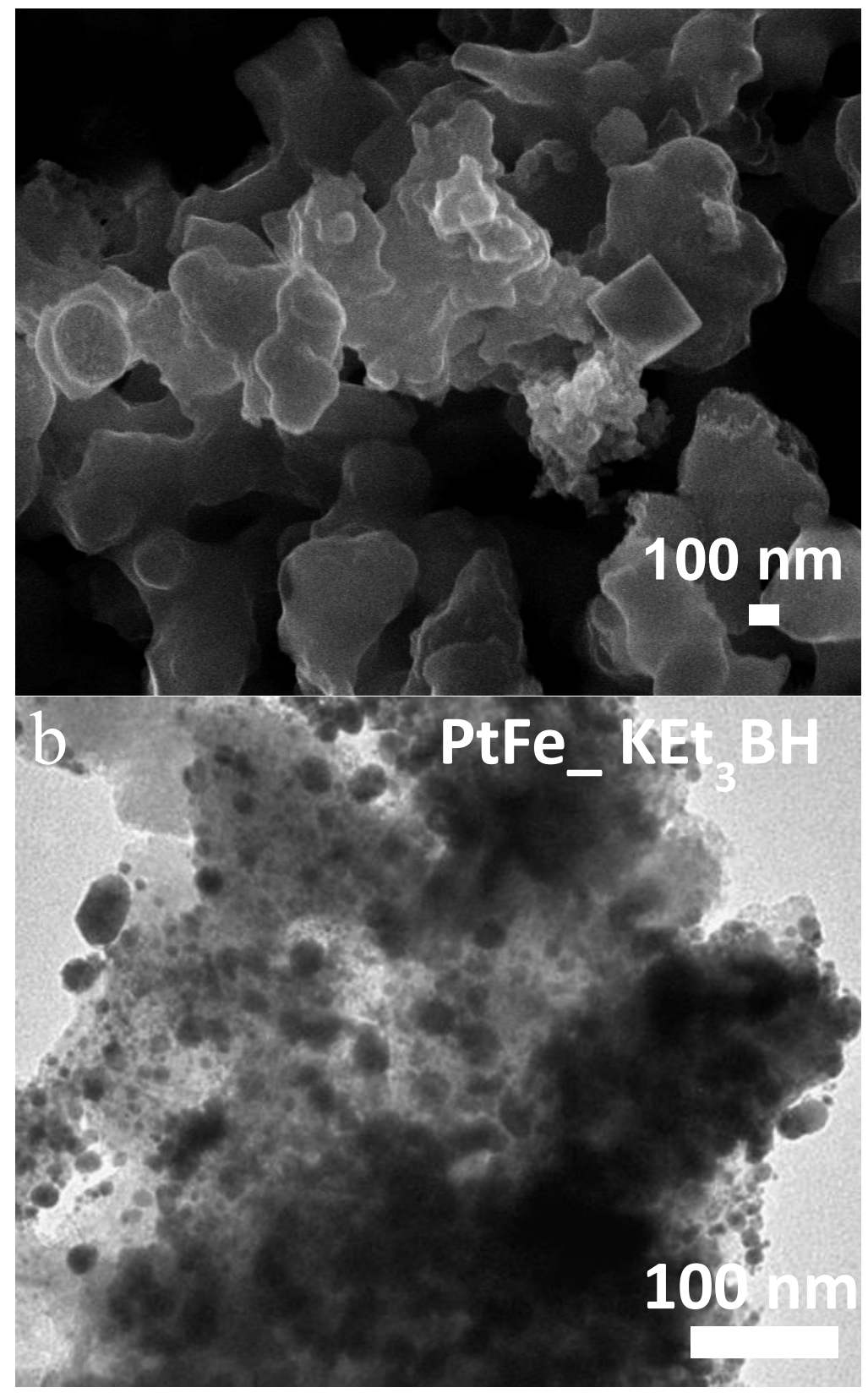

Figure 2.8: Scanning electron microscope (SEM) image for PtFe made with $\mathrm{LiEt}_{3} \mathrm{BH}$ annealed at $400{ }^{\circ} \mathrm{C}$ for 12 hours (a) and the TEM image of the PeFe made with $\mathrm{KEt}_{3} \mathrm{BH}$ annealed at $600{ }^{\circ} \mathrm{C}$ for 12 hours (b). 
interparticle diffusion of the PtFe. This behavior was consistently observed with other

Pt-M systems ${ }^{15}$ if using this method, suggesting it is fundamentally determined by the reducing agent and the formation of $\mathrm{KCl}$.

\subsection{Conclusion}

In this part of work, a detailed air and water free method to synthesize structurally ordered Pt-M nanoparticles has been discussed. In the synthesis method, the metal chlorides are used instead of organometallic precursors to minimize the organic contamination and with a availability of a wide range of metal precursor sources; THF is used as the solvent, which can provide a large stable potential window for different reducing agents; $\mathrm{LiEt}_{3} \mathrm{BH}$ and $\mathrm{KEt}_{3} \mathrm{BH}$ are used as reducing agents, which can provide strong reduction power and fast reduction kinetics. As we discussed, a wide range of metal chloride, either in the $\mathrm{MCl}_{\mathrm{x}}$ forms or in the $\mathrm{Li}_{\mathrm{x}} \mathrm{MCl}_{\mathrm{y}}$ forms, can be made with useful concentrations in THF solution and can be reduced with the suitable borohydride anions. The specific case of forming ordered PtFe by the $\mathrm{KCl}$ matrix annealing method weas discussed in enough detail to illustrate the method. One of the concerns on extending this process to other metals is the possible formation of metal hydrides when borohydrides are used as the reducing agent. However, a detailed theoretical analysis showed that the temperatures where the metal hydrides could be converted to Pt-M alloys are expected to be lower than the current annealing temperatures. Therefore, the synthesis system described in this chapter may in fact be a general synthetic method for intermetallic Pt-M particles. In the following chapters 
we show that this expectation can be met for certain Pt-M stoichiometries. We also show how the nano-particles produced by the $\mathrm{KCl}$ matric method can be transferred to suitable catalyst supports while preventing agglomeration of the nanoparticles.

In this Chapter, the investigation of suitable metal precursors and a facile synthesis method is a continuation of work previously persued by the former DiSalvo group members, especially Tanushree Ghosh, Brian Leonard, as well as current DiSalvo group members Minh Nguyen and Doug DeSario, along with a number of undergraduates who have worked with all of us. To develop the THF soluble metal chloride approach, Minh Nguyen and Professor DiSalvo have added considerably to making progress.

\section{REFERENCES}

(1) Bonet, F.; Grugeon, S.; Dupont, L.; Herrera-Urbina, R.; Guery, C.; Tarascon, J. M. J. Solid State Chem. 2003, 172, 111.

(2) Bonet, F.; Grugeon, S.; Dupont, L.; Herrera-Urbina, R.; TekaiaElhsissen, K.; Tarascon, J. M. Solid State Science 2002, 4, 665.

(3) Chen, J.; Herricks, T.; Geissler, M.; Xia, Y. J. Am. Chem. Soc. 2004, $126,10854$.

(4) Ducamp-Sanguesa, C.; Herrera-Urbina, R.; Figlarz, M. J. Solid State Chem. 1992, 100, 272. 
(5) Lu, P.; Dong, J.; Toshima, N. Langmuir 1999, 7980.

(6) Silvert, P. Y.; Tekaia-Elhsissen, K. Solid State Ionics 1995, 82, 53.

(7) Kissinger, P. T.; Heineman, W. R. Laboratory techniques in electroanalytical chemistry; 2nd ed.; Marcel Dekker, Inc.: New York, 1996.

(8) Roychowdhury, C.; Matsumoto, F.; Mutolo, P. F.; Abruna, H. D.; DiSalvo, F. J. Chem Mater 2005, 17, 5871.

(9) Roychowdhury, C.; Matsumoto, F.; Zeldovich, V. B.; Warren, S. C.; Mutolo, P. F.; Ballesteros, M.; Wiesner, U.; Abruña, H. D.; DiSalvo, F. J. Chem. Mater. 2006, 18, 3365.

(10) Bonnemann, H.; Richards, R. M. Eur. J. Inorg. Chem. 2001, 2455.

(11) Franke, R.; Rothe, J.; Pollmann, J.; Hormes, J.; Bo1nnemann, H.; Brijoux, W.; and Hindenburg, Th.; J. Am. Chem. Soc. 1996, 118, 12090.

(12) DiSalvo, F. J.; Casado-Rivera, E.; Volpe, D. J.; Alden, L.; Lind, C.; Downie, C.; Vazquez-Alvarez, T.; Angelo, A. C. D.; Abruna, H. D. J Am Chem Soc 2004, 126, 4043.

(13) Abe, H.; Matsumoto, F.; Alden, L. R.; Warren, S. C.; Abruna, H. D.; DiSalvo, F. J. J Am Chem Soc 2008, 130, 5452.

(14) DiSalvo, F. J.; Ghosh, T.; Leonard, B. M.; Zhou, Q. Chem Mater 2010, 22, 2190.

(15) Leonard, B. M.; Zhou, Q.; Wu, D. M.; DiSalvo, F. J. Chem Mater 2011, 23, 2499.

(16) Bard, A. J.; Faulkner, L. R. Electrochemical methods : fundamentals and applications; 2nd ed.; Wiley: New York, 2001.

(17) Adams, R. N. Abstr Pap Am Chem S 1982, 183, 46. 
(18) Cottrell, P. T.; Mann, C. K. J Electrochem Soc 1969, 116, 1499.

(19) Adams, R. N. Electrochemistry at solid electrodes; M. Dekker: New York, 1969.

(20) Sawyer, D. T.; Sobkowiak, A.; Roberts, J. L.; Sawyer, D. T. Electrochemistry for chemists; 2nd ed.; Wiley: New York, 1995.

(21) Garrett, D. E. Handbook of lithium and natural calcium chloride : their deposits, processing, uses and properties; 1st ed.; Elsevier Academic Press: Amsterdam ; Boston, 2004.

(22) Scherrer, P.; Göttinger Nachrichten Gesell., 1918, 2, 98.

(23) Patterson, A.; Phys. Rev. 1939, 56, 978. 


\section{CHAPTER 3}

\section{THE NANOPARTICLE-KCl (Np-KCl) METHOD}

PART I: THE SYNTHESIS

\subsection{Introduction}

\subsubsection{Synthetic criteria}

As we discussed in the earlier chapters, because of their tailored electronic structure and surface chemistry, the ordered intermetallic phases of Pt and transition metal bimetallic nanoparticles (Nps) are of great interest for polymer electrolyte membrane fuel cell (FEMFCs) applications ${ }^{12-4}$. This is especially relevant to the improvement of the catalytic activity of the oxygen reduction reaction (ORR), which is also the primary concern of this thesis. Recent studies already suggest that catalysts derived from the ordered intermetallic phases show better durability as well as higher catalytic activity. $^{5-8}$ In terms of the synthesis, there are three primary concerns for the intermetallic Pt-M nanoparticle catalysts, namely: forming the structurally ordered intermetallic phase; having the optimum particle size; and having a "clean" surface for catalytic reactions. Let's discuss these synthetic criteria one by one.

Firstly, to form the ordered intermetallic phase, as we discussed in chapter 1, a thermal annealing process is usually unavoidable. As shown in figure 2.5 and 2.6, the asprepared particles usually form in a disordered face centered cubic alloy phase, where the Pt and transition metal atoms are randomly distributed on specific sites (for FCC, 
Wycoff position 4a) in the structure. In order to form the ordered structure, where different metal atoms occupy specific sites, the atoms require sufficient thermal energy to diffuse across at least the diameter of one particle. Often ordering also requires the nucleation of a "seed" of the new ordered structure. For example, the cubic PtFe alloy must transform to the tetragonal ordered PtFe structure. As a result, a thermal annealing process is usually required to promoter the structural change.

Secondly, to provide a large surface area, the synthesis targets small particle sizes, typically in the range of 4 to $5 \mathrm{~nm}$. However, when the nanoparticle sizes are too small (smaller than $3 \mathrm{~nm}$ in the case of pure Pt), these particles go through dissolution, precipitation and coarsening, rendering poor stability ${ }^{9}$. A comprehensive theoretical study showed in the cathodic environment of PEMFCs, the particle morphology and size of Pt nanoparticles larger than $4 \mathrm{~nm}$ are relatively stable ${ }^{10}$. This result is also agrees with the experience of fuel cell R\&D testing by General Motors, where they reported optimal diameterss of 3 to $5 \mathrm{~nm}$.

Finally, a "clean" surface is needed for catalytic reaction to reach the highest rates. However, to achieve better size and/or shape control, for several decades the synthesis of Pt based bimetallic nanoparticles has relied on using different long chain surfactant oligomers. These strongly bound surfactants are used to control nanoparticle size as well as to prevent particle aggregation. There are two major concerns in terms of the synthesis of intermetallic nanoparticle catalysts using surfactants: 1) surfactants usually block surface reaction sites, hindering their catalytic activity; 2) thermal annealing of the nanoparticles is often required to develop atomic order or promote 
growth of certain facets. Strongly bound surfactants usually decompose rather than cleanly desorbing, leaving carbonaceous residues on the surface that deactivate the catalyst. Even if clean surfaces are obtained, aggregation of the nanoparticles at annealing temperatures leads to significant growth particle size, thus decreasing the active surface area per mass of catalyst.

3.1.2 Previous attempts to address the annealing challenge

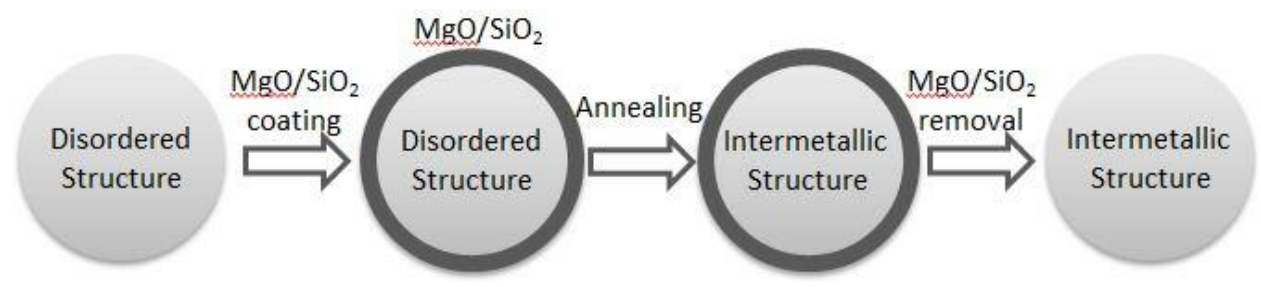

Figure 3.1: General scheme of the $\mathrm{MgO}$ and $\mathrm{SiO}_{2}$ matrix method.

To protect against particle size growth and agglomeration, a few alternative synthetic methods have been reported recently. Shown in figure 3.1 is a matrix method, where the nanoparticles were first synthesized using surfactants and then coated with inorganic matrix materials such as $\mathrm{MgO}^{8,11}$ or $\mathrm{SiO}_{2}{ }^{12-14}$. After the coating step, the nanoparticles were then annealed to the intermetallic phases and released for usage with the removal of the coating materials in acid or base. Since the melting temperatures of $\mathrm{MgO}$ and $\mathrm{SiO}_{2}$ are fairly high (above $1000{ }^{\circ} \mathrm{C}$ ), the nanoparticles are protected from aggregation and maintained small sizes during thermal treatment. However, there are two main drawbacks of this method. Firstly, the surfactants are still involved in early steps to provide the nanoparticles with specific sizes before 
coating step. These surfactants decompose on annealing, leaving residues on the catalyst surface. Secondly, to release the annealed nanoparticles, strong acids are usually required. For example, the $\mathrm{SiO}_{2}$ matrix method used $\mathrm{HF}$ to remove the $\mathrm{SiO}_{2}$ coating. The involvement of such acids increases the complexity of the method and may affect the surface composition of the annealed nanoparticles.

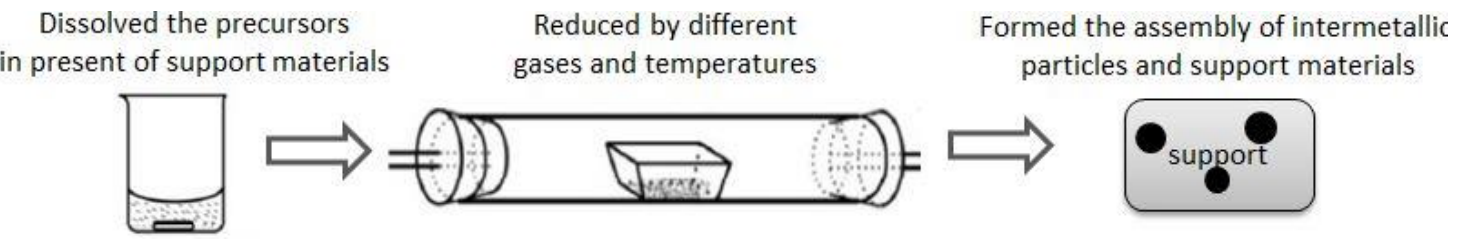

Figure 3.2: A general scheme for impregnation reduction method

Some solution phase chemical synthesis methods can provide intermetallic nanoparticles without using any surfactant ${ }^{5,6,15}$. For example, in a typical impregnation reduction method (figure 3.2), the metal precursors were dissociated into ions in the reaction solution in presence of the support materials, usually carbon black. The solution is stirred for a given period of time to allow the metal cations to be absorbed on the carbon black. The solution was then heated to elevated temperatures under reduction gases, usually hydrogen, to form alloys or ordered intermetallic phase. However, such methods do not usually permit independent control of particle size, phase, composition, and nanoparticle loading.

Another approach to achieve clean surfaces is based on using small molecules that are 
weak surfactants, such as ethylene glycol, as both the reducing agent and protecting ligand, which provides some control over particle size and composition ${ }^{16,17}$. In this case, ethylene glycol can be completely decomposed to gaseous products electrochemically at high potentials or by heat treatment below $175{ }^{\circ} \mathrm{C}$ in air or vacuum, leaving essentially clean surfaces. Generally, this process is suitable for producing nanoparticles of the more electronegative elements, such as $\mathrm{Au}, \mathrm{Pt}, \mathrm{Rh}$, $\mathrm{Ru}^{18,19}$. Alloys and intermetallic phases are difficult to prepare, since the reduction rates for different elements vary widely. In any case, ethylene glycol that binds to the metal surface is thought to be oxidized or decomposed under heat treatment, especially above $150{ }^{\circ} \mathrm{C}$, which makes it very challenging to anneal at higher temperatures to increase particle size, or to obtain an ordered intermetallic phase without significant particle agglomeration ${ }^{16}$.

As a short summary, for the purpose of making advanced nanoparticle catalysts for ORRs, previous methods do not provide a simultaneous control of small particle sizes, clean surfaces under the thermal annealing process, facile particle loading on the support materials or are not generally applicable to a variety of transition metals.

\subsection{The Np-KCl method}

3.2.1 The idea of the $\mathrm{Np}-\mathrm{KCl}$ method 
As we discussed in Chapter 2, using binary metal chloride or ternary lithium metal chloride as precursor, THF as solvent and triethylborohydrides as reducing agents, we proposed a general synthetic method for the synthesis of binary or even ternary intermetallic nanoparticles. The synthetic approach is surfactant free. However, to fulfill the synthetic criteria mentioned above, it has to be able to provide the desired particle sizes ( 3 to $5 \mathrm{~nm}$ ), after the thermal annealing process. In this chapter, a detailed investigation of this method will be provided.

3.2.2 Forming the $\mathrm{Np}-\mathrm{KCl}$ assembly I: the synthesis

Using $\mathrm{Pt}_{3} \mathrm{Fe}$ as an example, the full details of this $\mathrm{Np}-\mathrm{KCl}$ method will be discussed in the chapter. The formation of $\mathrm{Pt}_{3} \mathrm{Fe}$ nanoparticles and $\mathrm{KCl}$ salt in a THF solution is given in the following equation:

$3 \mathrm{PtCl}_{4}+\mathrm{FeCl}_{3}+15 \mathrm{KEt}_{3} \mathrm{BH} \rightarrow \mathrm{Pt}_{3} \mathrm{Fe}+15 \mathrm{KCl}+7.5 \mathrm{H}_{2}+15 \mathrm{Et}_{3} \mathrm{~B}$

(Equation 3.1)

$\mathrm{Pt}_{3} \mathrm{Fe}$ nanoparticles were formed in a very rapid solution based reduction of their chloride precursors by using THF as the solvent and $\mathrm{KEt}_{3} \mathrm{BH}$ as the reducing agent. One of the by-products, the $\mathrm{KCl}$ salt, is insoluble in $\mathrm{THF}^{20}$. Therefore, after the rapid (few seconds) injection of the reducing agent there are two nucleation and growth processes, one for $\mathrm{Pt}-\mathrm{Fe}$ nanoparticles and one for $\mathrm{KCl}$ nanocrystals. In the synthesis of $\mathrm{Pt}_{3} \mathrm{Fe}-15 \mathrm{KCl}$ (equation 3.1), after injection of $\mathrm{KEt}_{3} \mathrm{BH}$, a black precipitate was quickly formed (within a couple of seconds), leaving a clear supernatant. After 
decanting the supernatant, rinsing with THF, and vacuum drying, the precipitates were studied using a transmission electron microscope (TEM). As shown in figure 3.3a, the black dots are the Pt-Fe nanoparticles and the gray area is the $\mathrm{KCl}$. The average diameter for the $\mathrm{Pt}_{3} \mathrm{Fe}$ nanoparticles was $1.8 \mathrm{~nm}$ (figure $3.3 \mathrm{~b}$ ).
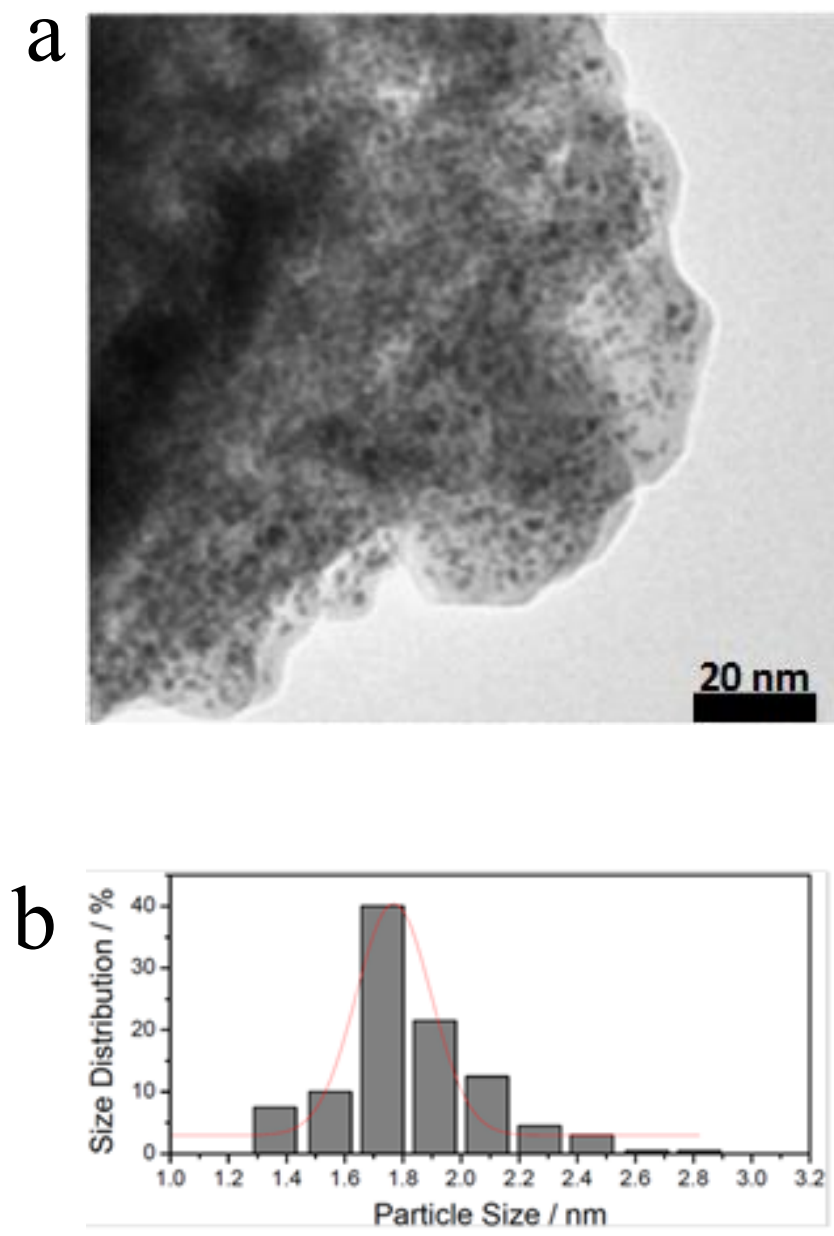

Figure 3.3: (a), A TEM image of the $\mathrm{Pt}_{3} \mathrm{Fe}-15 \mathrm{KCl}$ product obtained by room temperature reduction. (b), Size distribution of $\mathrm{Pt}_{3} \mathrm{Fe}$ nanoparticles in (a) 
To further explore the mechanism of the formation of the $\mathrm{Np}-\mathrm{KCl}$ matrix, two other experiments were conducted. First, we modified the synthesis of $\mathrm{Pt}_{3} \mathrm{Fe}-15 \mathrm{KCl}$ by cooling the metal precursor solution to minus $60{ }^{\circ} \mathrm{C}$. After injection of $\mathrm{KEt}_{3} \mathrm{BH}$, no precipitate formed, even after several hours at room temperature. The suspension remained black. A TEM image of a dried aliquot of the supernatant is shown in figure 3.4a. In contrast to the large Np-matrix agglomerations in the precipitated product obtained from room temperature reduction, unaggregated $\mathrm{Np}-\mathrm{KCl}$ assemblies were observed, many with $20 \mathrm{~nm}$ or smaller sizes. However, the average diameter of the $\mathrm{Pt}_{3} \mathrm{Fe}$ nanoparticles of this low temperature reduction was similar to those obtained from room temperature reduction. Another set of experiments was then designed to explore the nucleation and growth of $\mathrm{KCl}$ alone. A metathesis reaction of 15 equivalents of $\mathrm{LiCl}$ (which has a solubility of $4.6 \mathrm{wt} \%$ in $\mathrm{THF}^{21}$ ) with 15 equivalents of $\mathrm{KBEt}_{3} \mathrm{H}$ was performed at room temperature and at minus $60{ }^{\circ} \mathrm{C}$. In this case, only $\mathrm{KCl}$ nanoparticles should be formed as follows:

$$
15 \mathrm{KEt}_{3} \mathrm{BH}+15 \mathrm{LiCl} \rightarrow 15 \mathrm{KCl}+15 \mathrm{LiEt}_{3} \mathrm{BH}
$$

(Equation 3.2)

As shown in figure $3.4 \mathrm{~b}$, the product solution of the reaction conducted at $-60{ }^{\circ} \mathrm{C}$ was more transparent than the one at room temperature butprecipitation of $\mathrm{KCl}$ occurred much more rapidly at room temperature. These observations parallel those of the reactions that included metal precursors. All the above evidence suggests that the growth of $\mathrm{KCl}$ and $\mathrm{Pt}_{3} \mathrm{Fe}$ nanoparticles are two independent processes. 

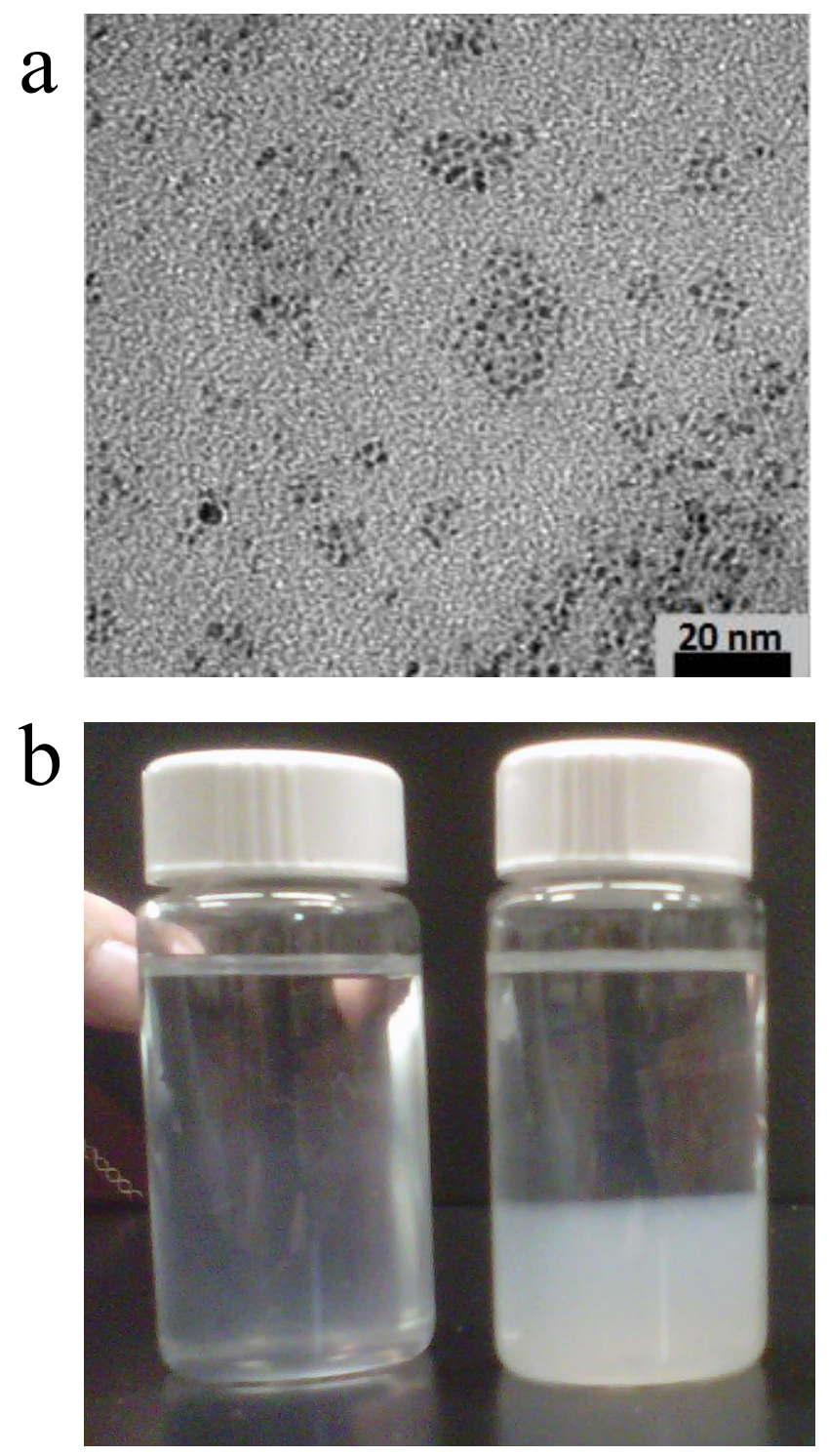

Figure 3.4: (a)A TEM image of a $\mathrm{Pt}_{3} \mathrm{Fe}-15 \mathrm{KCl}$ sample synthesized at $-60{ }^{\circ} \mathrm{C}$ and then warmed to room temperature looks different from figure 3.3 (a), in that much smaller $\mathrm{Np}-\mathrm{KCl}$ matrix particles are formed; (b) $\mathrm{LiCl}$ was reacted with $\mathrm{KEt}_{3} \mathrm{BH}$ at $-60{ }^{\circ} \mathrm{C}$ (left) and room temperature (right) to produce $\mathrm{KCl}$ particles. The $\mathrm{KCl}$ sol of the lower temperature reaction showed much more transparecy, suggesting, the $\mathrm{KCl}$ particles are much smaller and apparently do not aggregate quickly, even when warmed to room temperature. 


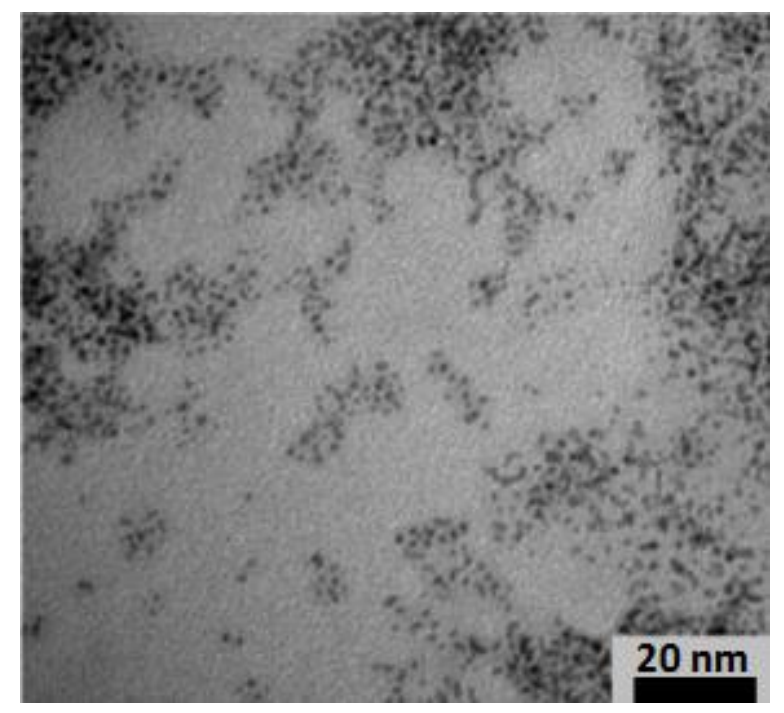

Figure 3.5: A TEM image of $\mathrm{Pt}_{3} \mathrm{Fe}-15 \mathrm{KCl}$ synthesized at room temperature in the presence of 65 equivalents of $\mathrm{LiEt}_{3} \mathrm{BH}$. As more $\mathrm{LiCl}$ is introduced, loosely aggregated but non-precipitating networks of small particles consisting of $\mathrm{Np}-\mathrm{KCl}$ matrix formed.

Since there is no surfactant in the solvent, as observed in our previous work ${ }^{22}$ as well as that of others ${ }^{23}$, the nanoparticles cannot be stabilized by THF alone, rather agglomeration followed by precipitation occurs. In this case, it is clear that at room temperature there are two rapid nucleation processes, by the time $\mathrm{Pt}-\mathrm{Fe}$ particles grow to only $2 \mathrm{~nm}$ or so in size, they interact with $\mathrm{KCl}$ to form a $\mathrm{Np}-\mathrm{KCl}$ matrix. Given the typical rates of agglomeration and precipitation of clean metal particles, these two processes must take place within seconds.

To confirm our hypothesized mechanism, another experiment was designed, in which 
all the experimental conditions were kept the same as for the sample $\mathrm{Pt}_{3} \mathrm{Fe}-15 \mathrm{KCl}$, but an excess of $\mathrm{LiCl}$ was added to the precursor salts. Although the detailed solubility data of $\mathrm{LiCl}$ in $\mathrm{KCl}$ nanocrystals at room temperature is lacking, it appears from bulk phase diagrams that the solubility is quite small ${ }^{24}$. Therefore there will be little or no $\mathrm{LiCl}$ mixed into the $\mathrm{Pt}_{3} \mathrm{Fe}-\mathrm{KCl}$ matrix. Thus, $\mathrm{Li}^{+}$and $\mathrm{Cl}^{-}$ions from the $\mathrm{LiCl}$ remain in solution after reaction. After reaction at room temperature, the supernatant was black in color, but no precipitation was observed. An aliquot of supernatant was dried and the TEM image is shown in figure 3.5. Small particles of the $\mathrm{Pt}_{3} \mathrm{Fe}-\mathrm{KCl}$ matrix are predominant. We hypothesize that the excess $\mathrm{Cl}^{-}$ions partly adsorb to the metal particles and perhaps to the $\mathrm{KCl}$, keeping the particles from aggregating due to electrostatic particle-particle repulsion.

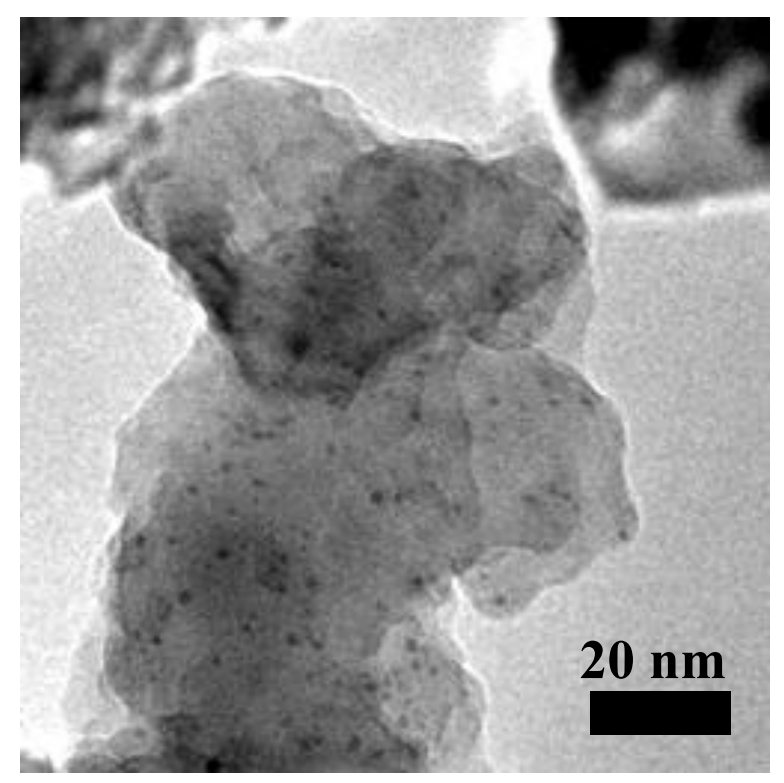

Figure 3.6: A TEM image of $\mathrm{Pt}_{3} \mathrm{Fe}-160 \mathrm{KCl}$ synthesized at room temperature. The small dark spots are the alloyed Pt-Fe nanoparticles. 
As shown in Eq. 3.3, the mole ratio of $\mathrm{Pt}_{3} \mathrm{Fe}$ to $\mathrm{KCl}$ can be altered by varying the amount of $\mathrm{LiCl}$ and $\mathrm{KBEt}_{3} \mathrm{H}$ added. For all $x$, no excess $\mathrm{Cl}^{-}$ions remain in the product solution. Rather all $\mathrm{Cl}^{-}$ions are scavenged from solution by the precipitating $\mathrm{KCl}$ and producing $\mathrm{LiEt}_{3} \mathrm{BH}$ :

$3 \mathrm{PtCl}_{4}+\mathrm{FeCl}_{3}+(15+x) \mathrm{KEt}_{3} \mathrm{BH}+x \mathrm{LiCl} \rightarrow \mathrm{Pt}_{3} \mathrm{Fe}+(15+x) \mathrm{KCl}+7.5 \mathrm{H}_{2}+$ $15 \mathrm{Et}_{3} \mathrm{~B}+x \mathrm{LiEt}_{3} \mathrm{BH} \quad$ (Equation 3.3)

$\mathrm{Pt}_{3} \mathrm{Fe}-40 \mathrm{KCl}, \mathrm{Pt}_{3} \mathrm{Fe}-80 \mathrm{KCl}$ and $\mathrm{Pt}_{3} \mathrm{Fe}-160 \mathrm{KCl}$ were made with $x=25,65$ and 145 , respectively. The TEM image of the as-made sample of $\mathrm{Pt}_{3} \mathrm{Fe}-160 \mathrm{KCl}$ is shown in figure 3.6. As the amount of $\mathrm{KCl}$ increases, the $\mathrm{Pt}_{3} \mathrm{Fe}$ nanoparticles are "diluted" in the $\mathrm{KCl}$ matrix. As shown in figure 3.6, distance between $\mathrm{Pt}_{3} \mathrm{Fe}$ nanoparticles increases considerably compared to that of the $\mathrm{Pt}_{3} \mathrm{Fe}-15 \mathrm{KCl}$.

The X-ray diffraction pattern of the as-synthesized Pt-Fe product obtained at room temperature, both before and after washing with water (in the presence or absence of $\mathrm{KCl}$ ), (figure 3.7) shows roughly $2 \mathrm{~nm}$ diameter domain sizes, which agrees with particle sizes calculated from the TEM images. In order to obtain larger particles and to develop an ordered crystal structure, the product must be annealed without particle sintering. 


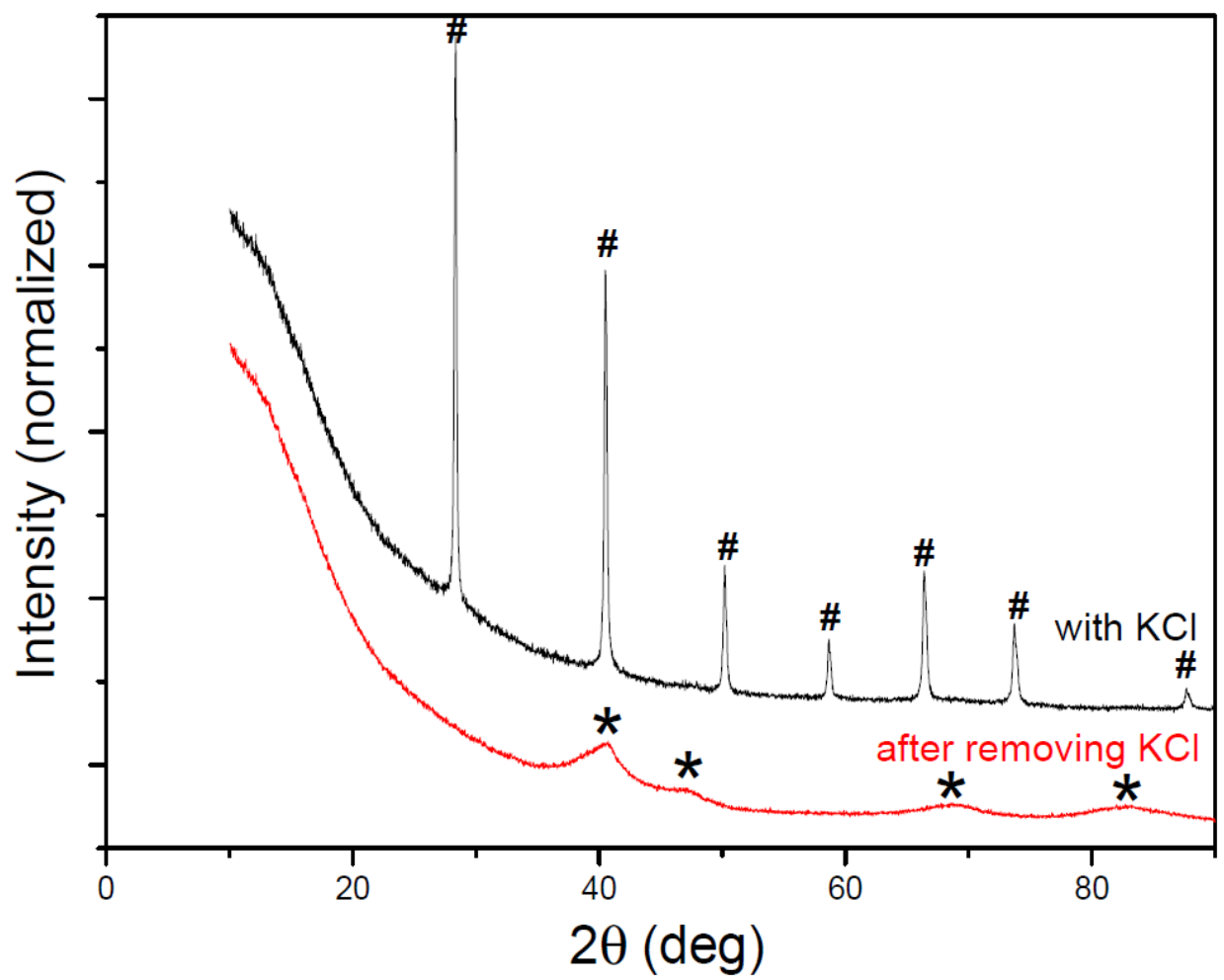

Figure 3.7: XRD patterns of as-synthesized Pt-Fe nanoparticles before (black) and after (red) removing $\mathrm{KCl}$. The peak positions for $\mathrm{KCl}$ are marked with \# and positions for Pt-Fe are marked with *. The Pt-Fe nanoparticle peaks are not visible before removing $\mathrm{KCl}$ since the nanoparticle peaks are weak and broad and $\mathrm{KCl}$ peaks are strong and sharp. Since the nanoparticles are relatively dilute within the $\mathrm{KCl}$ matrix, the broad nanoparticle peaks are not seen in the XRD of the $\mathrm{Np}-\mathrm{KCl}$ sample. The broadening for Pt-Fe nanoparticle peaks shows the as-reduced Pt-Fe nanoparticles have very small diameters of about $2 \mathrm{~nm}$. 


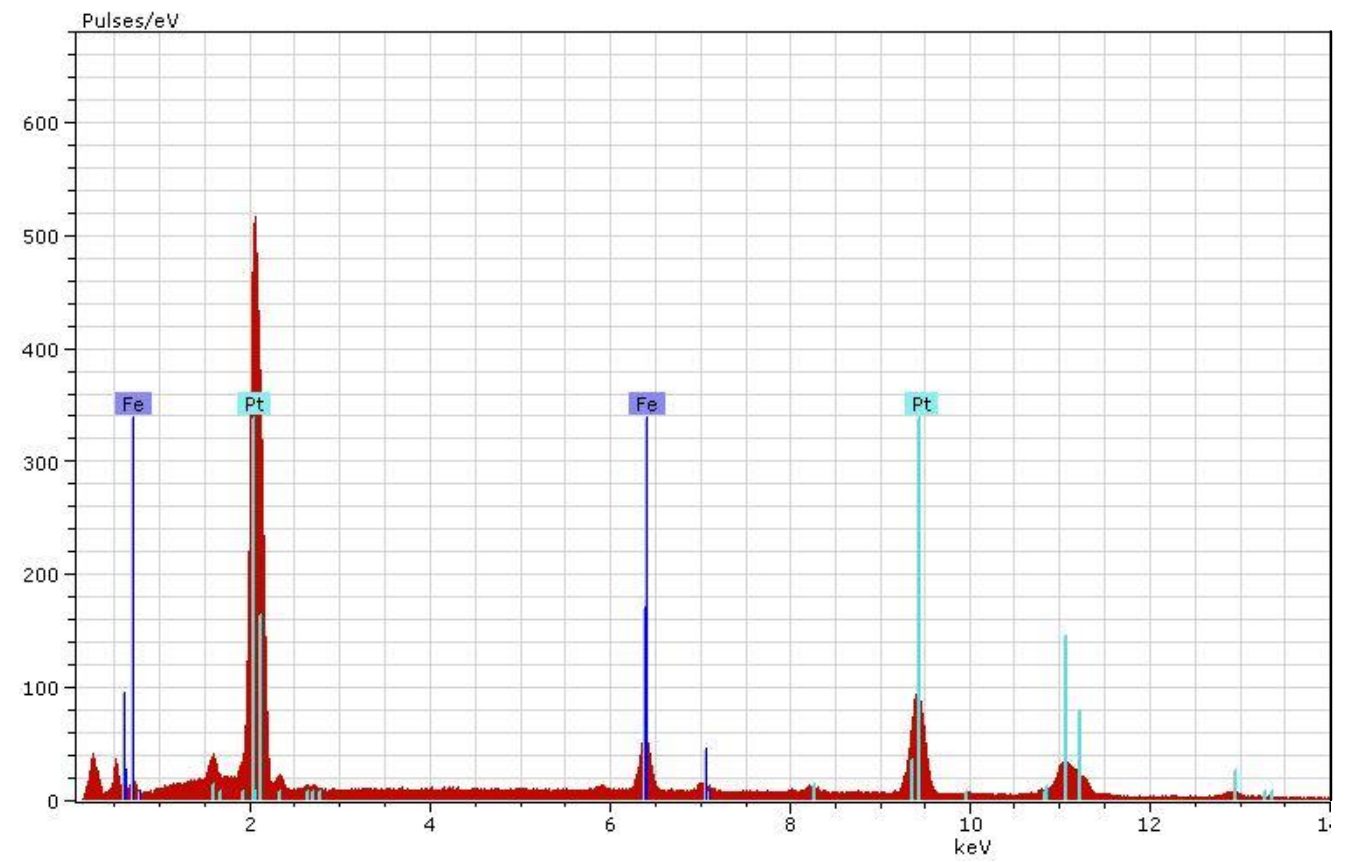

Figure 3.8: After thermal annealing, the $\mathrm{Pt}_{3} \mathrm{Fe}-80 \mathrm{KCl}$ sample was washed by water to dissolve the $\mathrm{KCl}$ salt. The subsequent EDX study of this sample showed the mole ratio of $\mathrm{Pt}$ to $\mathrm{Fe}$ is 75:25 $( \pm 3 \%)$ and uniform throughout the sample. However, EDX samples an area of about one micron square, so this is an average over very many particles.

As expected, the average composition of an ensemble of nanoparticles in an area of $500 \mathrm{~nm}$ by $500 \mathrm{~nm}$ obtained by Energy-dispersive X-ray spectroscopy (EDX) for Pt:Fe was typically 75:25 ( $\pm 3 \%)$ and uniform throughout the sample (figure 3.8).

3.2.3 Forming the $\mathrm{Np}-\mathrm{KCl}$ assembly II: the anions

As shown in equation 3.3 , the $\mathrm{Np}-\mathrm{KCl}$ assembly can be formed for any value of $x$ (at 
least up to $x=145$ ). However, as the value of $x$ increases, the concentration of the borohydride anions left in solution increases, since in all the synthesis reactions mentioned above, the final solution volume was kept at $25 \mathrm{ml}$. Since the THF and $\mathrm{Et}_{3} \mathrm{~B}$ bind very weakly to metal surfaces and thus are not expected to provide stabilization effects ${ }^{23}$, the only component left that could stabilize the $\mathrm{Np}-\mathrm{KCl}$ assembly is the $\mathrm{Et}_{3} \mathrm{BH}^{-}$.

Indeed, as shown in equation 3.4 and table 3.1, where the PtFe nanoparticles were synthesized in a same fashion as $\mathrm{Pt}_{3} \mathrm{Fe}$, when the value of $x$ is 14 or smaller, a black precipitate quickly formed leaving a clear or very light brownish supernatant. When the $x$ is higher than 14 , some black precipitate still formed; however, the color of the supernatant became darker. When the $x$ value is 20 or larger, no precipitate formed, instead the solution turns black immediately and the $\mathrm{Np}-\mathrm{KCl}$ assemblies are stabilized and suspended in the solution. Such results suggest that as $x$ increased, the concentration of $\mathrm{Et}_{3} \mathrm{BH}^{-}$increased, which then provided a larger stabilization effect. As a result, with increasing $\mathrm{x}$, a larger fraction of small assemblies of the $\mathrm{Np}-\mathrm{KCl}$ were able to be stabilized and the supernatant became darker. Further increases of $x$ eventually suspended all the $\mathrm{Np}-\mathrm{KCl}$ assemblies that were generated through the reduction.

In a parallel experiment, no extra $\mathrm{Et}_{3} \mathrm{BH}^{-}$was generated; instead, extra $\mathrm{LiCl}$ was added without increasing the amount of $\mathrm{KEt}_{3} \mathrm{BH}$, so that $\mathrm{Cl}^{-}$was the only anion remaining (equation 3.5) to stabilize the $\mathrm{Np}-\mathrm{KCl}$ assemblies. However, in this case, instead of a value of 14 or higher, only a small excess of that anion ( $x$ at 2 or even 
lower), produces a clear stabilization effect, such that the $\mathrm{Np}-\mathrm{KCl}$ assembly remains suspended in the solution (table 3.2). Such results suggest that the interaction of Np$\mathrm{KCl}$ with $\mathrm{Cl}^{-}$is stronger than that with $\mathrm{Et}_{3} \mathrm{BH}^{-}$.

The experiments described above to add extra $\mathrm{Cl}^{-}$anions to the reaction solution are difficult to precisely titrate. This is in part due to the fact that some excess reducing agent $(10 \%$ to $50 \%)$ is usually used to address several issues: 1 . The concentration of reducing agent in the received reagent varies somewhat and likely declines during use of the reagent due to reaction with impurities in the glove box atmosphere, 2. Residual water or other reducable impurities in the THF or on the flask walls will react with the borohydride, reducing the amount available to react with the metal precursors, $3.5 \mathrm{ml}$ syringes are used to inject the reducing agent, but can be read to abut $+/-5 \%$ of the desired volume, and 4, when catalyst supports are also suspended in the THF (vide infra), extra reducing agent is needed because their surfaces usually contain acidic hydrogen from $\mathrm{OH}, \mathrm{COOH}$, etc. In this case, when $x$ is small, the $\mathrm{Cl}^{-}$might be all consumed by the $\mathrm{K}^{+}$from the excess reducing agent, and results in a considerable error in determining the actual $\mathrm{Cl}^{-}$concentration. Therefore, a further investigation of even smaller values of $x$ was not conducted and the $\mathrm{Cl}^{-}$anion results should be considered semi-quantitative and the $x$ values an upper bound.

The result of another set of experiments also suggests that the $\mathrm{Et}_{3} \mathrm{BH}^{-}$is indeed a much weaker binding agent with the $\mathrm{Np}-\mathrm{KCl}$ assemblies (equation 3.6 and table 3.3). In this experiment, as shown in equation 3.5 , no $\mathrm{LiCl}$ was added while the rest was the same as the first set of experiments. That is, the counter ion for the $\mathrm{Et}_{3} \mathrm{BH}^{-}$is $\mathrm{K}^{+}$ instead of $\mathrm{Li}^{+}$. As a result, the $\mathrm{Np}-\mathrm{KCl}$ is only precipitated out of the solution with $\mathrm{x}$ 
higher than 33. By changing $\mathrm{Li}^{+}$to $\mathrm{K}^{+}$as counter ion, a stronger cation-anion interaction is introduced, since $\mathrm{K}^{+}$is only weakly solvated by THF (compared to $\mathrm{Li}^{+}$) which makes the $\mathrm{Et}_{3} \mathrm{BH}^{-}$less available to coordinate to the $\mathrm{Np}-\mathrm{KCl}$. As a result, the concentration of dissociated anion was lower and no longer could stabilize $\mathrm{Np}-\mathrm{KCl}$ in solution.

$\mathrm{PtCl}_{4}+\mathrm{FeCl}_{3}+(7+x) \mathrm{KEt}_{3} \mathrm{BH}+x \mathrm{LiCl} \rightarrow$

$\mathrm{PtFe}+(7+x) \mathrm{KCl}+3.5 \mathrm{H}_{2}+7 \mathrm{Et}_{3} \mathrm{~B}+x \mathrm{LiEt}_{3} \mathrm{BH}$

(Equation 3.4)

\begin{tabular}{|c|c|c|}
\hline$x$ value & $\begin{array}{c}\text { Product: } \\
\mathrm{PtFe}+3.5 \mathrm{H}_{2}+7 \mathrm{Et}_{3} \mathrm{~B}+\end{array}$ & $\begin{array}{c}\text { The Np-KCl assembly } \\
\text { precipitated or suspended }\end{array}$ \\
\hline 14 & $21 \mathrm{KCl}+14 \mathrm{Li}^{+}+14 \mathrm{Et}_{3} \mathrm{BH}^{-}$ & Precipitated \\
\hline 20 & $27 \mathrm{KCl}+20 \mathrm{Li}^{+}+20 \mathrm{Et}_{3} \mathrm{BH}^{-}$ & Suspended \\
\hline 25 & $32 \mathrm{KCl}+25 \mathrm{Li}^{+}+25 \mathrm{Et}_{3} \mathrm{BH}^{-}$ & Suspended \\
\hline 33 & $40 \mathrm{KCl}+33 \mathrm{Li}^{+}+33 \mathrm{Et}_{3} \mathrm{BH}^{-}$ & Suspended \\
\hline
\end{tabular}

Table 3.1: The product of the reaction according to equation 3.4 and the effect of $x$ on the precipitation of a $\mathrm{Np}-\mathrm{KCl}$ matrix product. 
$\mathrm{PtCl}_{4}+\mathrm{FeCl}_{3}+7 \mathrm{KEt}_{3} \mathrm{BH}+x \mathrm{LiCl} \rightarrow$

$\mathrm{PtFe}+7 \mathrm{KCl}+3.5 \mathrm{H}_{2}+7 \mathrm{Et}_{3} \mathrm{~B}+x \mathrm{LiCl}$

(Equation 3.5)

\begin{tabular}{|c|c|c|}
\hline$x$ value & $\begin{array}{c}\text { Product: } \\
\mathrm{PtFe}+3.5 \mathrm{H}_{2}+7 \mathrm{Et}_{3} \mathrm{~B}+\end{array}$ & $\begin{array}{c}\text { The Np-KCl assembly } \\
\text { precipitated or suspended }\end{array}$ \\
\hline 2 & $7 \mathrm{KCl}+2 \mathrm{Li}^{+}+2 \mathrm{Cl}^{-}$ & Suspended \\
\hline 3 & $7 \mathrm{KCl}+3 \mathrm{Li}^{+}+3 \mathrm{Cl}^{-}$ & Suspended \\
\hline 4 & $7 \mathrm{KCl}+4 \mathrm{Li}^{+}+4 \mathrm{Cl}^{--}$ & Suspended \\
\hline 7 & $7 \mathrm{KCl}+7 \mathrm{Li}^{+}+7 \mathrm{Cl}^{--}$ & Suspended \\
\hline
\end{tabular}

Table 3.2: The product of the reaction according to equation 3.5 and the effect of $x$ on the precipitation of a $\mathrm{Np}-\mathrm{KCl}$ matrix product

$\mathrm{PtCl}_{4}+\mathrm{FeCl}_{3}+(7+x) \mathrm{KEt}_{3} \mathrm{BH} \rightarrow$

$\mathrm{PtFe}+7 \mathrm{KCl}+3.5 \mathrm{H}_{2}+7 \mathrm{Et}_{3} \mathrm{~B}+x \mathrm{KEt}_{3} \mathrm{BH}$

(Equation 3.6)

\begin{tabular}{|c|c|c|}
\hline$x$ value & $\begin{array}{c}\text { Product: } \\
\mathrm{PtFe}+3.5 \mathrm{H}_{2}+7 \mathrm{Et}_{3} \mathrm{~B}+\end{array}$ & $\begin{array}{c}\text { The Np-KCl assembly } \\
\text { precipitated or suspended }\end{array}$ \\
\hline 14 & $7 \mathrm{KCl}+14 \mathrm{~K}^{+}+14 \mathrm{Et}_{3} \mathrm{BH}^{-}$ & Precipitated \\
\hline 20 & $7 \mathrm{KCl}+20 \mathrm{~K}^{+}+20 \mathrm{Et}_{3} \mathrm{BH}^{-}$ & Precipitated \\
\hline 25 & $7 \mathrm{KCl}+25 \mathrm{~K}^{+}+25 \mathrm{Et}_{3} \mathrm{BH}^{-}$ & Precipitated \\
\hline 33 & $7 \mathrm{KCl}+33 \mathrm{~K}^{+}+33 \mathrm{Et}_{3} \mathrm{BH}^{-}$ & Precipitated \\
\hline
\end{tabular}

Table 3.3: The product of the reaction according to equation 3.6 and the effect of $x$ on the precipitation of a $\mathrm{Np}-\mathrm{KCl}$ matrix product 
3.2.4 Synthesis of the intermetallic phase with controlled sizes
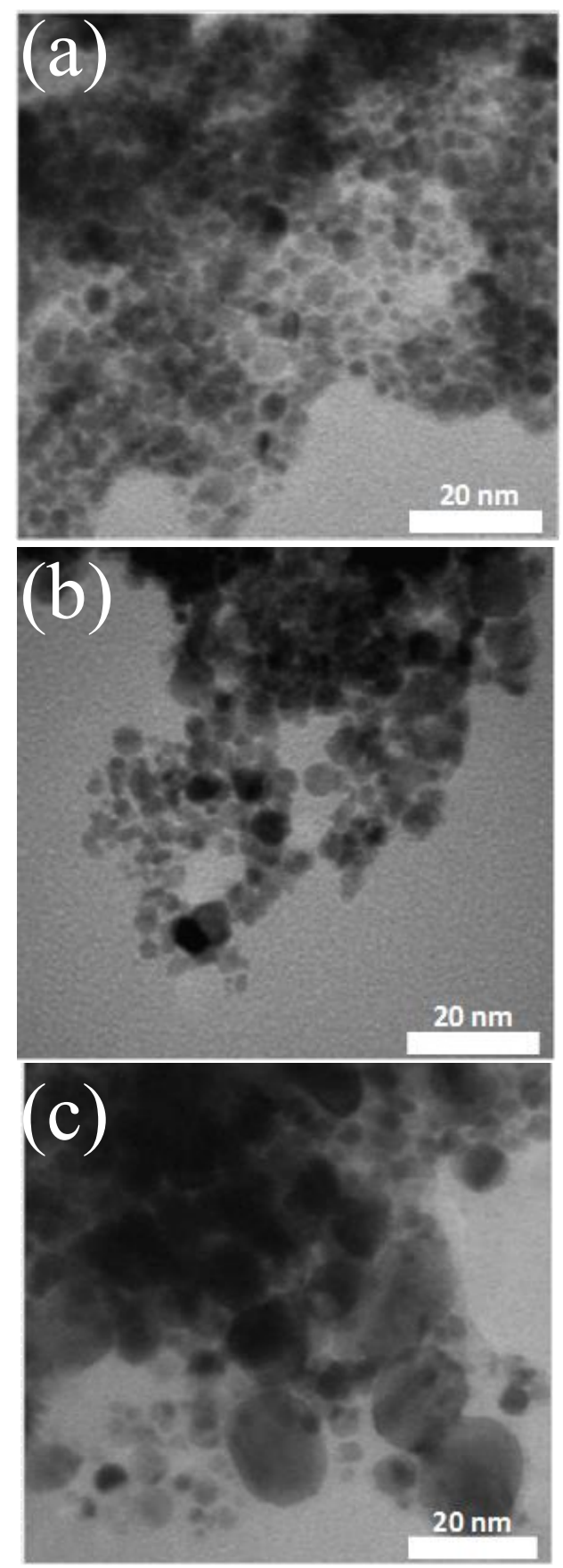

Figure 3.9: TEM images of $\mathrm{Pt}_{3} \mathrm{Fe}-40 \mathrm{KCl}$ annealed at (a) $400{ }^{\circ} \mathrm{C}$, (b) $500{ }^{\circ} \mathrm{C}$ and (c) $600{ }^{\circ} \mathrm{C}$ for 12 hours. 
After the $\mathrm{Np}-\mathrm{KCl}$ assemblies were formed, they were then sealed in a silica tube under vacuum and thermally annealed at $600{ }^{\circ} \mathrm{C}$ for 12 hours. Here $\mathrm{Pt}_{3} \mathrm{Fe}-15 \mathrm{KCl}-600{ }^{\circ} \mathrm{C}$ $12 \mathrm{hr}, \mathrm{Pt}_{3} \mathrm{Fe}-40 \mathrm{KCl}-600{ }^{\circ} \mathrm{C}-12 \mathrm{hr}, \mathrm{Pt}_{3} \mathrm{Fe}-80 \mathrm{KCl}-600{ }^{\circ} \mathrm{C}-12 \mathrm{hr}$, and $\mathrm{Pt}_{3} \mathrm{Fe}-160 \mathrm{KCl}-600$ ${ }^{\circ} \mathrm{C}-12 \mathrm{hr}$ were studied to explore the effect of the $\mathrm{KCl}$ content on the annealed nanoparticle products. For samples with the highest $\mathrm{KCl}$ content, the size of $\mathrm{Pt}_{3} \mathrm{Fe}$ nanoparticles increased by only factors of $\sim 2$ upon thermal annealing. In comparison, when no extra salt is present, the domain size, as determined by $\mathrm{X}$-ray diffraction, increased by a factor of $4 \sim 5$ and significant interparticle sintering occured.

Not surprisingly, we observe that the annealing temperature and the molar ratio of $\mathrm{Np}$ to $\mathrm{KCl}$ plays an important role in the final particle sizes after thermal annealing.

The TEM images of $\mathrm{Pt}_{3} \mathrm{Fe}-40 \mathrm{KCl}$ annealed at $400{ }^{\circ} \mathrm{C}, 500{ }^{\circ} \mathrm{C}$ and $600{ }^{\circ} \mathrm{C}$ for 12 hours are shown in figure $3.9 \mathrm{a}$ to $3.9 \mathrm{c}$ respectively. As the annealing temperature increases, the particle sizes increase. Since the particle-particle coalescence is the main mechanism for growth (discussed in the following chapter), the $\mathrm{Pt}_{3} \mathrm{Fe}$ nanopaticles must have some mobileity in the $\mathrm{KCl}$ matrix. At the same temperature, we expect that smaller particles would have a higher mobility. We consider the as-made $1.8 \mathrm{~nm} \mathrm{Pt}_{3} \mathrm{Fe}$ as "building blocks". During thermal annealing, those building blocks coalesced together in or on the surface of the $\mathrm{KCl}$ matrix and formed larger particles. Once larger particles are formed, we presume that they become less mobile. As the temperature increases, the mobility of particles with larger sizes increases and allows particle growth to continue at an observable rate. 

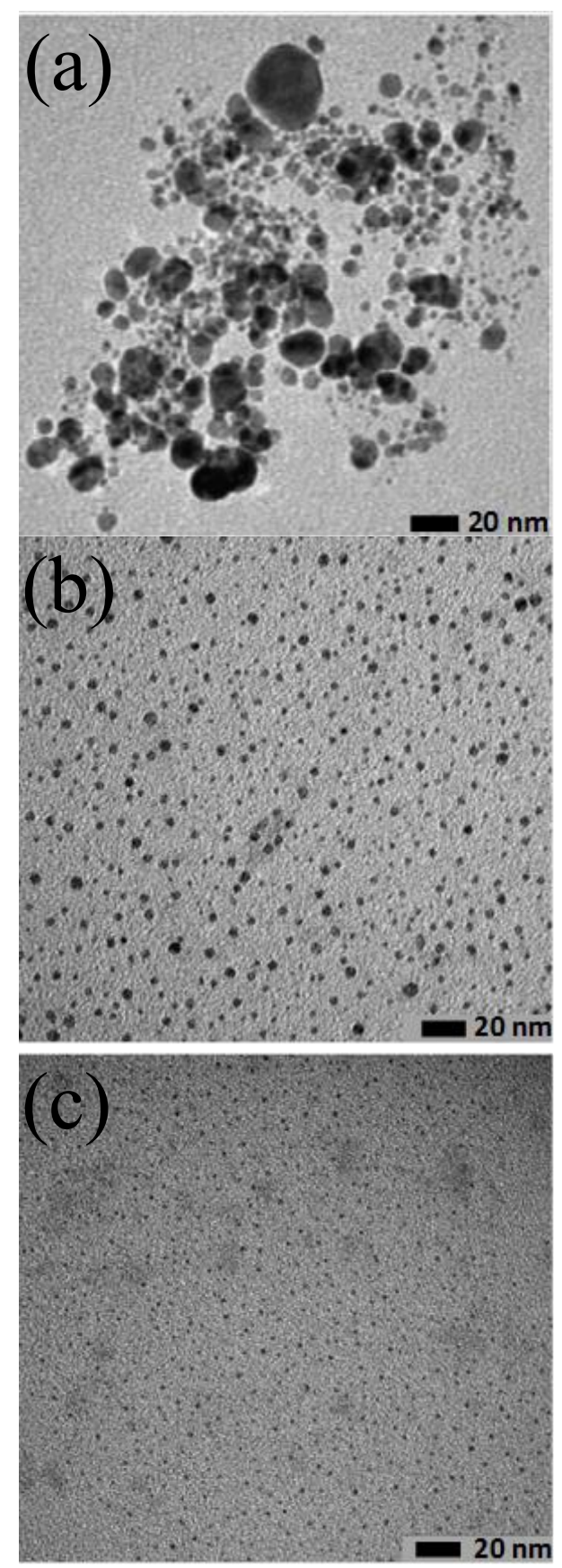

Figure 3.10: TEM images of (a) $\mathrm{Pt}_{3} \mathrm{Fe}-15 \mathrm{KCl}$, (b) $\mathrm{Pt}_{3} \mathrm{Fe}-80 \mathrm{KCl}$, and (c) $\mathrm{Pt}_{3} \mathrm{Fe}-160 \mathrm{KCl}$, annealed at $600{ }^{\circ} \mathrm{C}$ for 12 hours. 
To explore the effects of changing the molar ratio of $\mathrm{Pt}_{3} \mathrm{Fe}$ to $\mathrm{KCl}$ on the $\mathrm{Pt}_{3} \mathrm{Fe}$ agglomeration and sintering, we needed to remove the $\mathrm{KCl}$. Ethylene glycol turned out to be ideal for this purpose, since $\mathrm{KCl}$ is soluble and since metal particles are stabilized (prevented from further agglomeration) in ethylene glycol. ${ }^{17}$ A portion of the dried $\mathrm{Np}-\mathrm{KCl}$ matrix was sonicated in an 80:20 solution of ethylene glycol and water, then a small drop of the suspended particles were quickly transferred to a TEM grid. The TEM image of the desalted $\mathrm{Pt}_{3} \mathrm{Fe}-15 \mathrm{KCl}-600{ }^{\circ} \mathrm{C}-12 \mathrm{hr}$ sample is shown in figure 3.10a. Particle sizes vary from $2 \mathrm{~nm}$ to larger than $20 \mathrm{~nm}$. There was essentially no size control in this case. On the other hand, the TEM image of the desalted $\mathrm{Pt}_{3} \mathrm{Fe}-$ $80 \mathrm{KCl}-600{ }^{\circ} \mathrm{C}-12 \mathrm{hr}$ sample showed a much narrower particle size distribution (figure $3.10 \mathrm{~b})$. The majority of particle diameters are between 3 and $6 \mathrm{~nm}$, with an average particle size of $4 \mathrm{~nm}$ based on statistics from determining the diameters of more than 200 particles (figure 3.11a). Similarly, as shown in figure 3.10c, further increasing the molar ratio of $\mathrm{KCl}: \mathrm{Pt}_{3} \mathrm{Fe}$ to 160: 1 results in even smaller particle sizes after thermal annealing. An average particle size of ca. $2.5 \mathrm{~nm}$ is obtained, based on statistics from more than 250 particles from $\mathrm{Pt}_{3} \mathrm{Fe}-160 \mathrm{KCl}$ annealed at $600{ }^{\circ} \mathrm{C}$ for 12 hours (figure 3.11b). Notice that in figure 3.3a and figure 3.6, the $\mathrm{Pt}_{3} \mathrm{Fe}$ nanoparticles are similar in size as the as-made (room temperature) samples.

With a higher molar ratio of metal precursors to $\mathrm{KCl}$, the nanoparticles are very close to each other. At $600{ }^{\circ} \mathrm{C}$, which is close to the melting temperature of $\mathrm{KCl}\left(771{ }^{\circ} \mathrm{C}\right)$, the rate of agglomeration in the $\mathrm{KCl}$ matrix is apparently high enough to produce significant particle growth. However, with a lower particle density in the $\mathrm{KCl}$ matrix, the particles are efficiently separated and particle growth is much slower. 

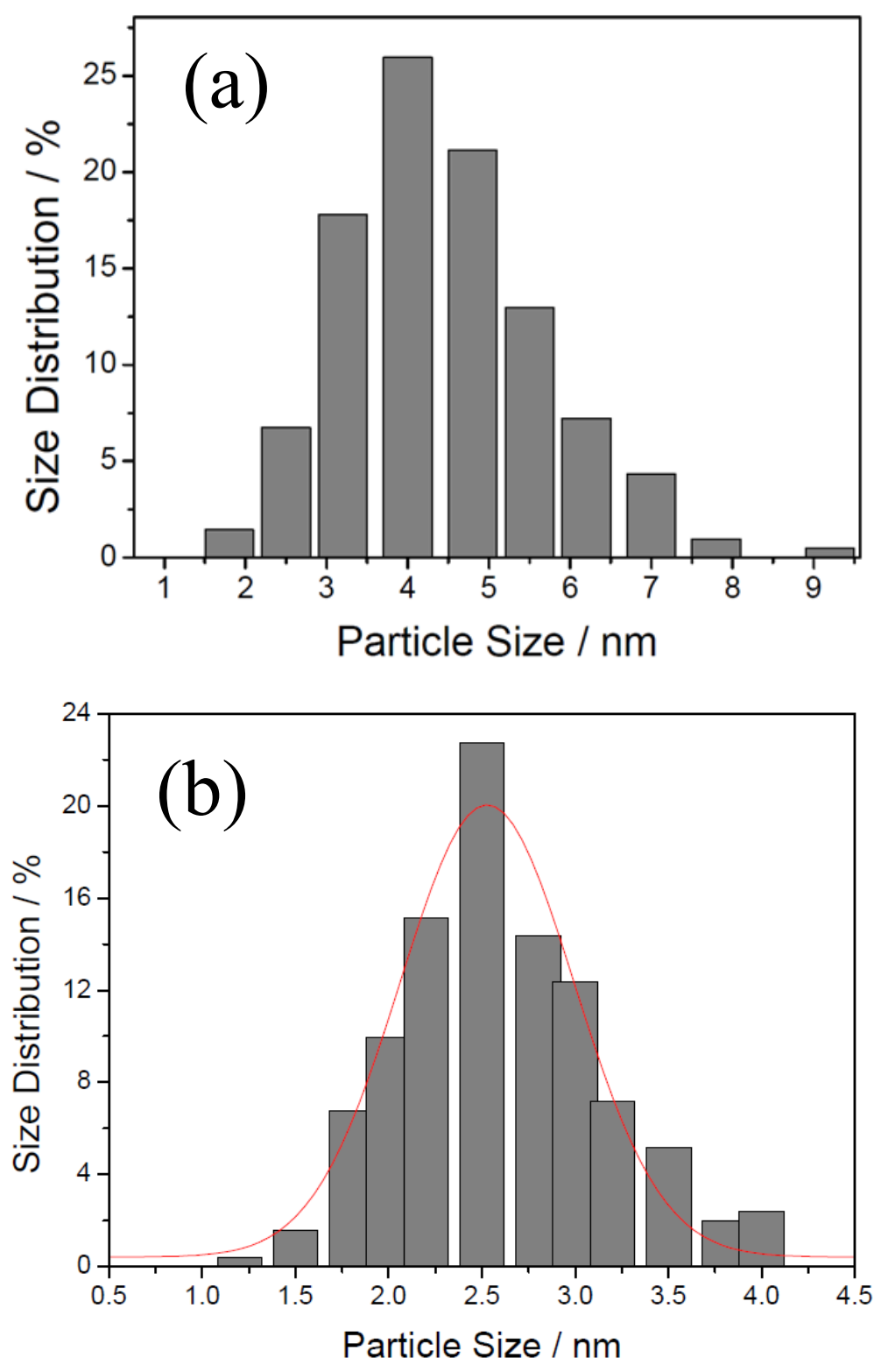

Figure 3.11: (a) Size distribution of nanoparticles of $\mathrm{Pt}_{3} \mathrm{Fe}-80 \mathrm{KCl}-600{ }^{\circ} \mathrm{C}-12 \mathrm{hr}$. Based on figure $3.10 \mathrm{~b}$, more than two hundred nanoparticles were analyzed. The average nanoparticle size was $4 \mathrm{~nm}$. Standard deviation is $1.2 \mathrm{~nm}$. (b) Size distribution of $\mathrm{Pt}_{3} \mathrm{Fe}-160 \mathrm{KCl}-600{ }^{\circ} \mathrm{C}-12 \mathrm{hr}$. Based on figure $3.10 \mathrm{c}$, more than two hundred and fifty nanoparticles were analyzed. The average nanoparticle size was $2.5 \mathrm{~nm}$. 


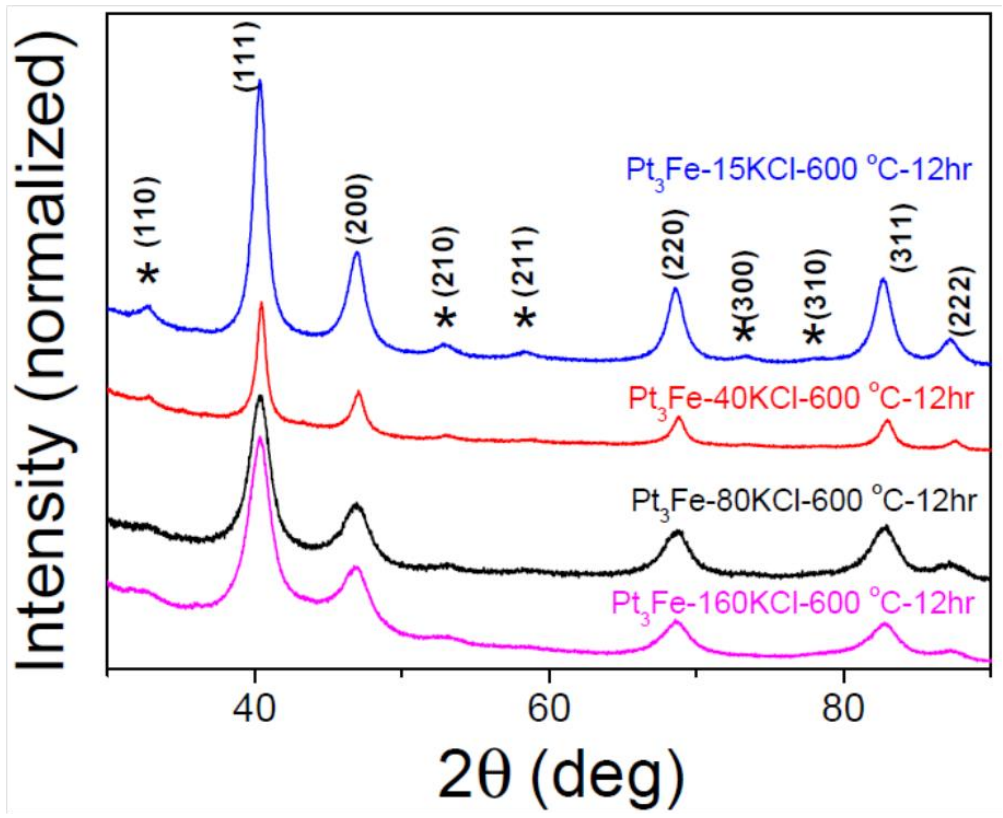

Figure 3.12: $\mathrm{XRD}$ patterns of all four samples of $\mathrm{Pt}_{3} \mathrm{Fe}-15 \mathrm{KCl}(\mathrm{blue}), \mathrm{Pt}_{3} \mathrm{Fe}-$ $40 \mathrm{KCl}(\mathrm{red}), \mathrm{Pt}_{3} \mathrm{Fe}-80 \mathrm{KCl}$ (black) and $\mathrm{Pt}_{3} \mathrm{Fe}-160 \mathrm{KCl}$ (purple) annealed at $600{ }^{\circ} \mathrm{C}$ for 12 hours, show the $\mathrm{Pt}_{3} \mathrm{Fe}$ intermetallic phase. The peak positions for the ordered intermetallic phase of $\mathrm{Pt}_{3} \mathrm{Fe}$ are marked with *. These weak ordered superlattice peaks are visible in all four samples. 
XRD patterns of the four annealed samples are shown in figure 3.12. The only peaks observed are indexed by the ordered cubic structure of $\mathrm{Pt}_{3} \mathrm{Fe}$. No peaks corresponding to $\mathrm{Pt}, \mathrm{Fe}$, other intermetallics in the Pt-Fe phase diagram (such as the 1:1 PtFe intermetallic phase), or any other Pt- or Fe-containing compounds are present in the diffraction pattern. The observation of ordering peaks, such as the expected weak (110), (210) and (211) peaks, demonstrates that the desired ordered intermetallic phase rather than the disordered alloy phase of Pt-Fe was prepared for all four samples. Based on the Scherrer equation, an average crystal domain size of $4 \mathrm{~nm}$ and $3.4 \mathrm{~nm}$ are calculated from the line widths of the XRD patterns for $\mathrm{Pt}_{3} \mathrm{Fe}-80 \mathrm{KCl}-600{ }^{\circ} \mathrm{C}-12 \mathrm{hr}$ and $\mathrm{Pt}_{3} \mathrm{Fe}-160 \mathrm{KCl}-600{ }^{\circ} \mathrm{C}-12 \mathrm{hr}$, respectively.

The particle sizes calculated from TEM images of $\mathrm{Pt}_{3} \mathrm{Fe}-160 \mathrm{KC}-600{ }^{\circ} \mathrm{C}-12 \mathrm{hr}$ are only slightly smaller than the value calculated from XRD, indicating that each particle is a single crystal. The small difference may due to the volume-weighted nature of XRD measurements, which tend to overestimate the geometric particle size. ${ }^{25}$ Since the particle sizes for $\mathrm{Pt}_{3} \mathrm{Fe}-160 \mathrm{KCl}-600{ }^{\circ} \mathrm{C}-12 \mathrm{hr}$ are around $2.5 \mathrm{~nm}$, the XRD peaks of which are very broad. To better study these small ordered intermetallic nanoparticles, we have also evaluated the atomic level distribution of $\mathrm{Pt}$ and $\mathrm{Fe}$ in $\mathrm{Pt}_{3} \mathrm{Fe}-160 \mathrm{KCl}$ annealed at $600{ }^{\circ} \mathrm{C}$ by performing annular dark-field STEM (ADF-STEM) and electron-energy loss spectroscopy (EELS) imaging. The sample was washed with water to remove $\mathrm{KCl}$ and then transferred to a TEM grid for TEM and EELS studies. 


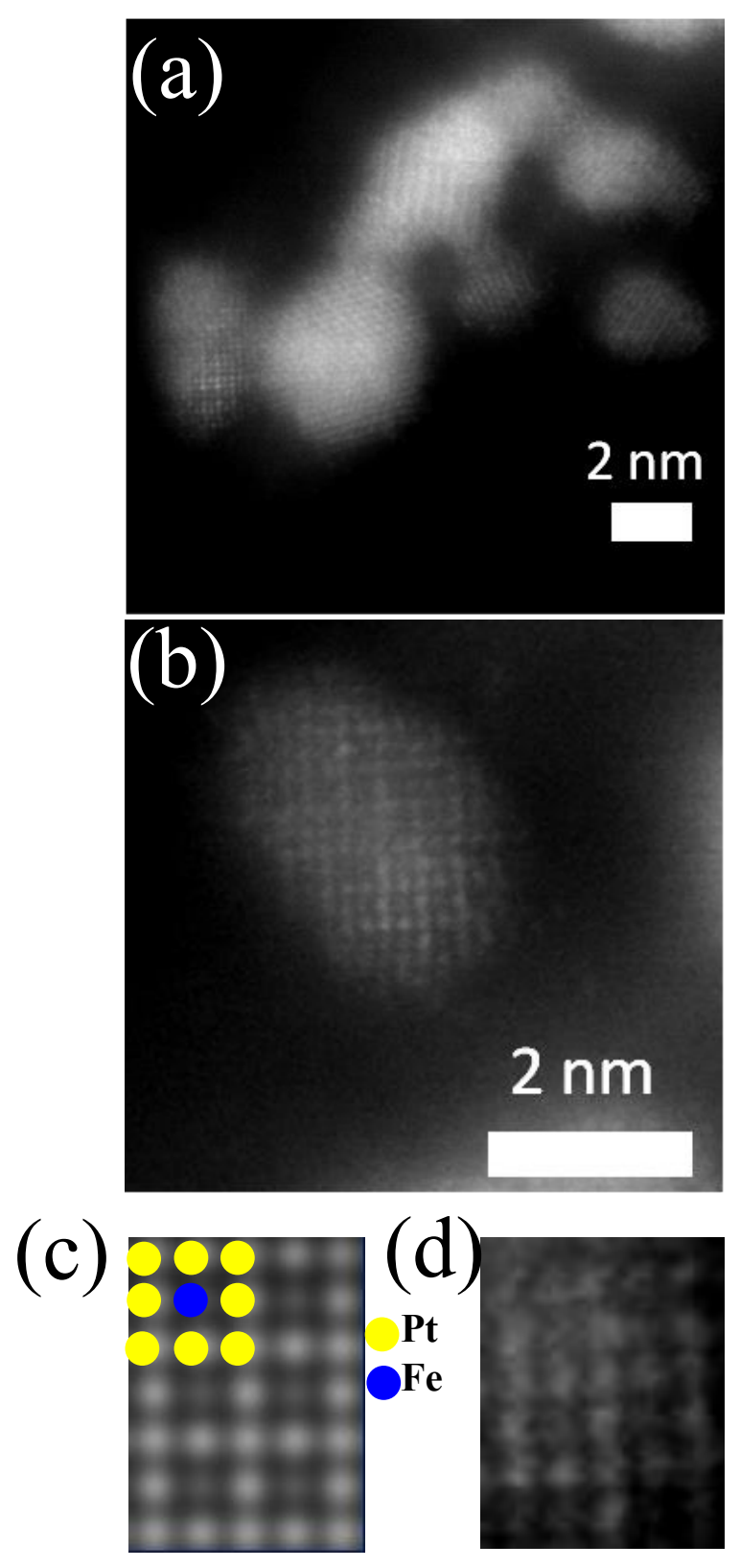

Figure 3.13: (a) Annular dark-field STEM (ADF-STEM) overview image of the thermally annealed ordered $\mathrm{Pt}_{3} \mathrm{Fe}$ nanoparticles. (b) An ADF-STEM image of an ordered $\mathrm{Pt}_{3} \mathrm{Fe}$ particle projected along the [100] axis. (c) A model ADF-STEM image of the projection of an ordered $\mathrm{Pt}_{3} \mathrm{Fe}$ particle along [100] axis. (d) An expanded view of a section of the super lattice features from (b). 
An ADF-STEM overview image of a representative region is shown in figure 3.13a. In figure 3.13b, a $2.5 \mathrm{~nm} \mathrm{Pt}_{3} \mathrm{Fe}$ nanoparticle is viewed along the [100] zone axis. Due to the unique super periods that are not present in the disordered alloy phase, the $\mathrm{L}_{2}$ ordered intermetallic structure in $\mathrm{Pt}_{3} \mathrm{Fe}$ can be directly identified. For example, for the ordered intermetallic particles, along the [100] axis, the projected unit cell is composed of a periodic square array of pure Fe columns surrounded by Pt columns at the edges and corners of each unit cell (figure 3.13c). Because of the ' $Z$-contrast' of Pt and $\mathrm{Fe}$, the Pt columns will have intensity higher than that of the Fe columns in ADFSTEM images (figure 3.13a, b). ${ }^{26,27}$ This differs from the disordered alloy phase, which has all columns with random distribution of atoms. A demonstration of such ordered structure along the [100] axis is shown in figure 3.13c. A cropped area of an experimental ADF-STEM image of the particle in figure 3.13b shows a similar pattern (figure 3.13d) suggesting the ordered structure has been achieved. This ordered intermetallic structure can also be observed along other zone axes. In figure 3.14a, an ordered nanoparticle with a $2.5 \mathrm{~nm}$ diameter is viewed along the [211] zone axis. The lattice spacings of $2.7 \AA$ and $2.2 \AA$ are attributed to (110) and (111) lattice fringes; both values are matched with the bulk $\mathrm{Pt}_{3} \mathrm{Fe}$ intermetallic structure. The fast Fourier transform (FFT) pattern of this nanoparticle is shown in figure 3.14b; the diffraction spots from the $\{110\}$ and $\{210\}$ superlattice reflections clearly show that the ordered structure was achieved. To further confirm the ordering, we have simulated the diffraction patterns of an ideal $\mathrm{Pt}_{3} \mathrm{Fe}$ fcc cyrstal along its [211] zone axis, and the simulated result in figure $3.14 \mathrm{c}$ matches the experimental result in figure $3.14 \mathrm{~b}$. This is conclusive evidence that the intermetallic structure is stable even in ultrasmall sized (c.a. $3 \mathrm{~nm}$ ) nanoparticles after thermal annealing. 


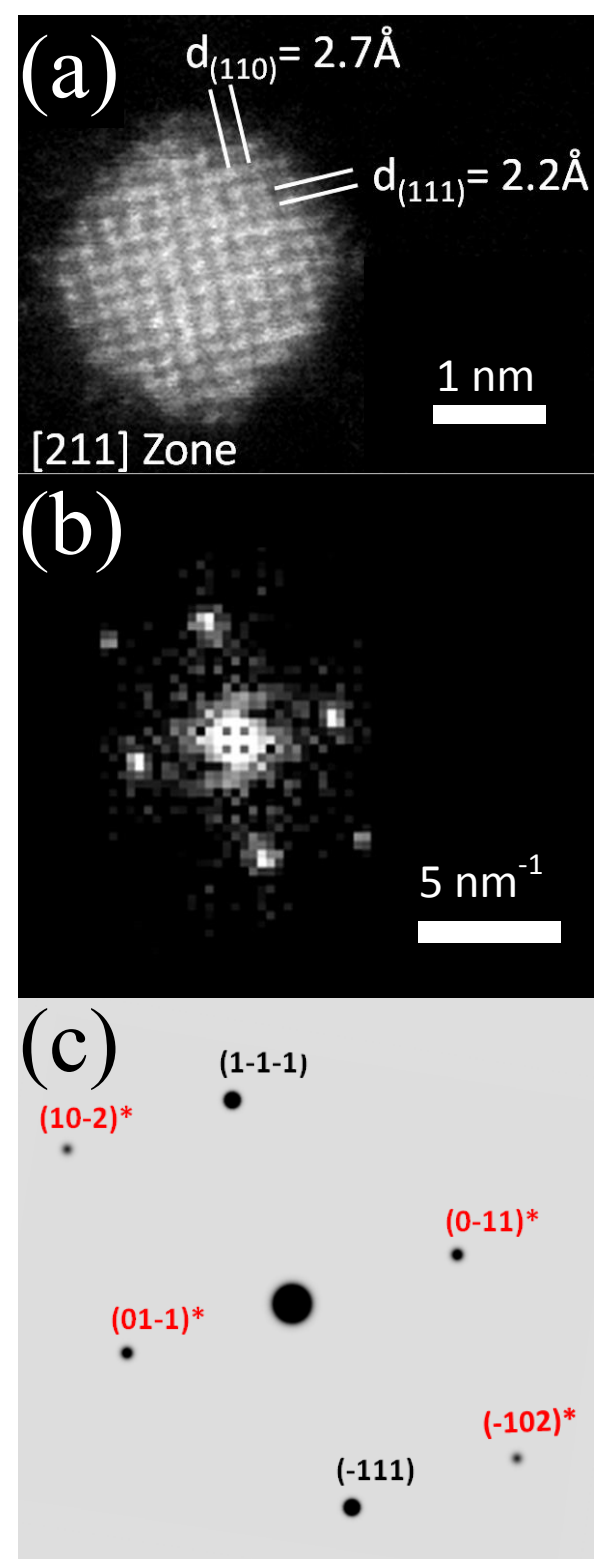

Figure 3.14: (a) HAADF-STEM image of one $\mathrm{Pt}_{3} \mathrm{Fe}$ nanoparticle on [211] zone axis, with (b) diffraction pattern showing the intermetallic superlattice peaks of $\{110\}$ and $\{210\}$. (c) Simulated diffraction pattern (contrast inverted) from [211] zone axis, which matches the experimental diffraction pattern in (i). The intermetallic peaks are in red and marked by *. 


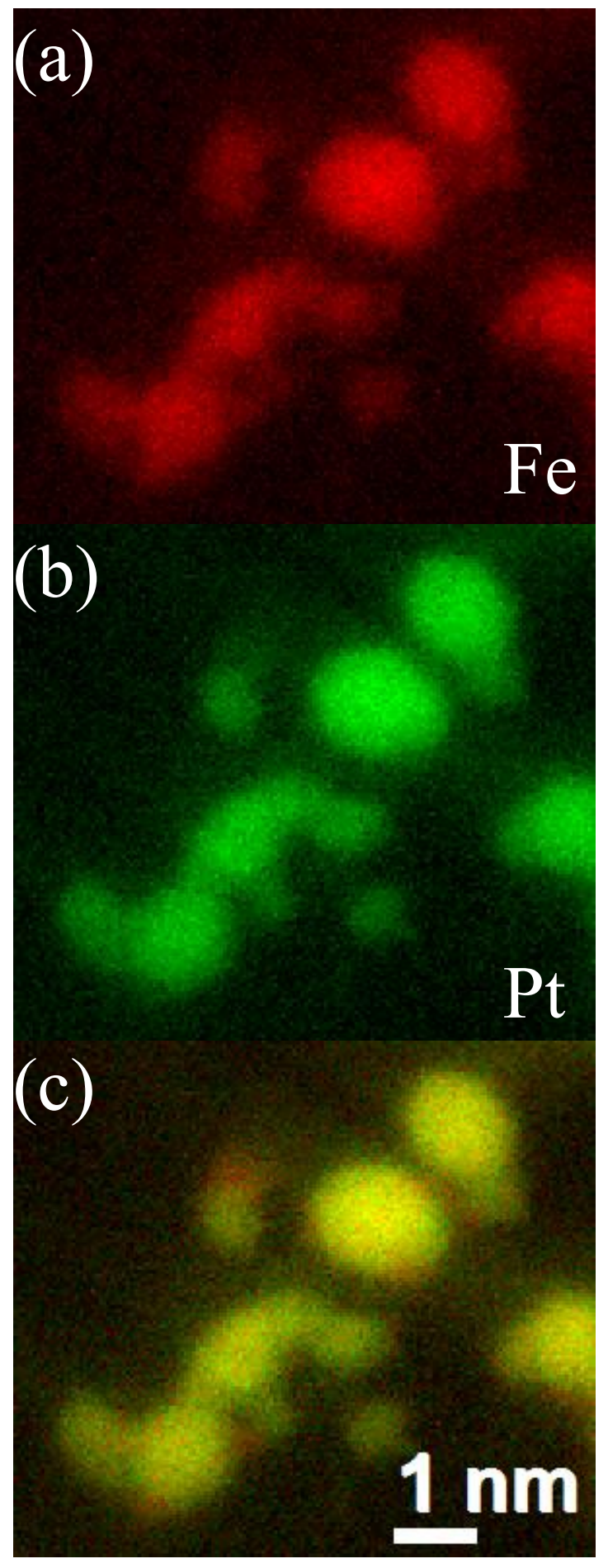

Figure 3.15: The 2-D EELS maps of figure 3.13a are shown in, (a) for Fe and (b) for Pt along with the (c) composite Fe vs. Pt map. 
Based on the ADF-STEM images along with the appearance of superlattice peaks in XRD (figure 3.12), we confirm that at least a majority of the annealed particles are atomically ordered. The EELS maps of Fe, Pt and their composite of the particle in figure 3.13a are shown in figure 3.15a, 3.15b and 3.15c respectively. The signals from Fe are extracted by using a power-law background fit of the Fe- $\mathrm{L}_{2,3}$ edge, since the peaks have distinct and abrupt onsets. However, due to the delayed onset and breadth of the Pt-M and Pt-N edges, we have used a MCR (multivariate component resolution) method to extract a Pt- $\mathrm{N}_{3}$ edge. The reliability of this method for the Pt- $\mathrm{N}_{3}$ edge has been verified in our previous publications. ${ }^{28}$ From the EELS images, a homogenous distribution of $\mathrm{Fe}$ and $\mathrm{Pt}$ is observed, with no obvious phase segregation (figure 3.15ac).

\subsection{The growth mechanism of bimetallic nanoparticles in the $\mathrm{KCl}$ matrix during thermal annealing}

3.3.1 The method for studying the growth mechanism of nanoparticles in the $\mathrm{KCl}$ matrix during thermal annealing

As we discussed above, the $\mathrm{Np}-\mathrm{KCl}$ method could be used to synthesize intermetallic Pt-M nanoparticles with somewhat controlled particle sizes. As shown in figure 3.3a and figure 3.6, the nanoparticles are similar in size for the as-made (room temperature) samples. However, by varying the molar ratio of Pt-M and $\mathrm{KCl}$, after thermal annealing at $600{ }^{\circ} \mathrm{C}$ for 12 hours, the one with the more salt rendered smaller particle sizes. This phenomenon was rationalized by the effect of diluting the "building 
blocks", where the as-prepared Pt-M nanoparticles are considered as the "building blocks". As a result, the more the "building blocks" are diluted in the matrix the smaller the expected size of the particle after thermal annealing. However, to further improve this method, especially to better control the particle sizes after thermal annealing, the growth mechanism of the nanoparticles or "building blocks" during the thermal treatment need to be understood. Here, the synthesis of intermetallic PtFe nanoparticles with $4 \mathrm{~nm}$ using this $\mathrm{Np}-\mathrm{KCl}$ method will be presented as an example, and the details of the particle growth mechanism will be discussed.

Nanoparticle sintering on support materials has been studied for decades, both experimentally and theoretically, most of which were interpreted by classical Ostwaldripening models. In this model, individual metal atoms leave a nanoparticle or a cluster, diffuse through on the support materials and join another particle. However, different from the Ostwald ripening mechanism, in the Coalescence model, the growth of particle sizes results from the motion of the nanoparticle themselves. Due to the improvement of the electron microscope technology, some recent studies could provide direct observation of the particle sintering process by in-situ STEM (scanning transmission electron microscope) method ${ }^{29}$. Therefore, the in-situ STEM was utilized as the main approach to identify the mechanism for particle growth in the $\mathrm{Np}-\mathrm{KCl}$ method.

The details of the in-situ STEM experiment are listed as below. In-situ heating experiments were obtained on a Schottky-field-emission-gun Tecnai F20 scanning transmission electron microscope (STEM) operated at $200 \mathrm{keV}$. A high-angle annular 
dark field detector provided an incoherent projection image of the specimen with a signal intensity proportional to the amount of material and its atomic number, which is also known as Z-contrast. The in-situ heating substrate (E-AHA21) and the heating TEM holder (Aduro System) are manufactured by Protochips, Inc. The heating chip contains a resistive ceramic membrane so the set temperature can be attained in less than a second, after changing the set point. The calibration of temperatures was performed by using a thermo-camera prior to the experiment, and the stability of temperature was determined to be $+/-1{ }^{\circ} \mathrm{C}$. The high resolution STEM images and EELS (electron-energy loss spectroscopy) maps were acquired on a $5^{\text {th }}$-order aberration-corrected scanning transmission electron microscope (Nion UltraSTEM) operated at $100 \mathrm{kV}$, with a convergence angle $\alpha_{\max }=\sim 30 \mathrm{mrad}$. With the setup, atomic-resolution imaging and EELS mapping are achieved routinely. ${ }^{29}$

3.3.2 The synthesis of $4 \mathrm{~nm}$ intermetallic PtFe nanoparticles using the $\mathrm{Np}-\mathrm{KCl}$ method

Here, to study the mechanism, let's start with the synthesis of a similar system, intermetallic PtFe. As discussed in the earlier chapter, the PtFe nanoparticles are prepared through a one-pot scalable synthesis and stabilization method. Instead of separate synthesis and protection steps, a nanoparticle- $\mathrm{KCl}(\mathrm{Np}-\mathrm{KCl})$ matrix is formed in a simple room temperature liquid phase reduction in THF (Equation 3.7).

$$
\begin{aligned}
& \mathrm{PtCl}_{4}+\mathrm{FeCl}_{3}+33 \mathrm{LiCl}+40 \mathrm{KEt}_{3} \mathrm{BH} \rightarrow \\
& \mathrm{PtFe}+40 \mathrm{KCl}+3.5 \mathrm{H}_{2}+7 \mathrm{Et}_{3} \mathrm{~B}+33 \mathrm{LiEt}_{3} \mathrm{BH}
\end{aligned}
$$


Samples of $\mathrm{Np}-\mathrm{KCl}$ assemblies were annealed for 12 hours at $400{ }^{\circ} \mathrm{C}$ and $600{ }^{\circ} \mathrm{C}$, respectively. As expected, the average composition of an ensemble of nanoparticles in an area of $500 \mathrm{~nm}$ by $500 \mathrm{~nm}$ obtained by EDX for Pt:Fe is typically 50:50 ( $\pm 3 \%)$ and uniform throughout the sample (figure 3.16).

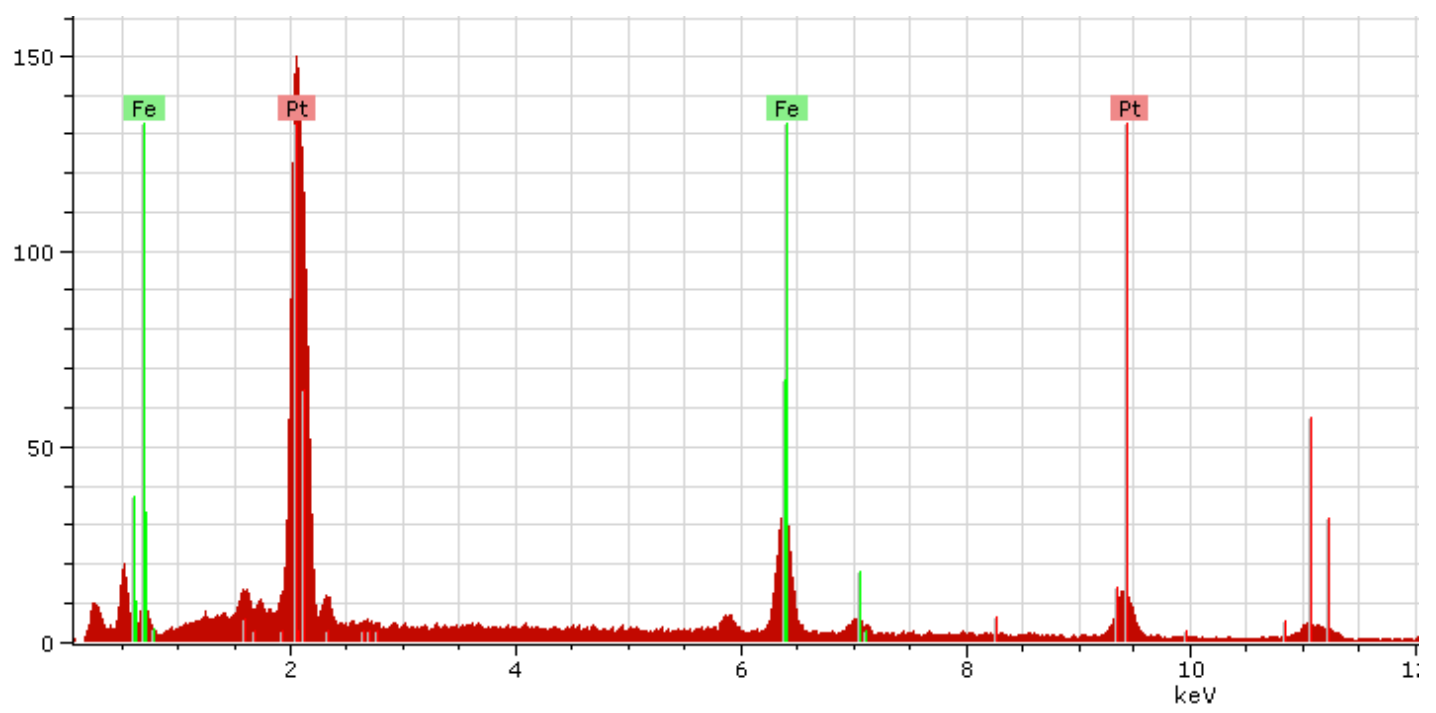

Figure 3.16: After thermal annealing, the PtFe-40KCl sample was washed with water to dissolve the $\mathrm{KCl}$. The subsequent energy-dispersive $\mathrm{X}$-ray spectroscopy (EDX) study of this sample showed that the mole ratio of Pt to Fe was typically 50:50 ( $\pm 3 \%)$ and uniform throughout the sample. 


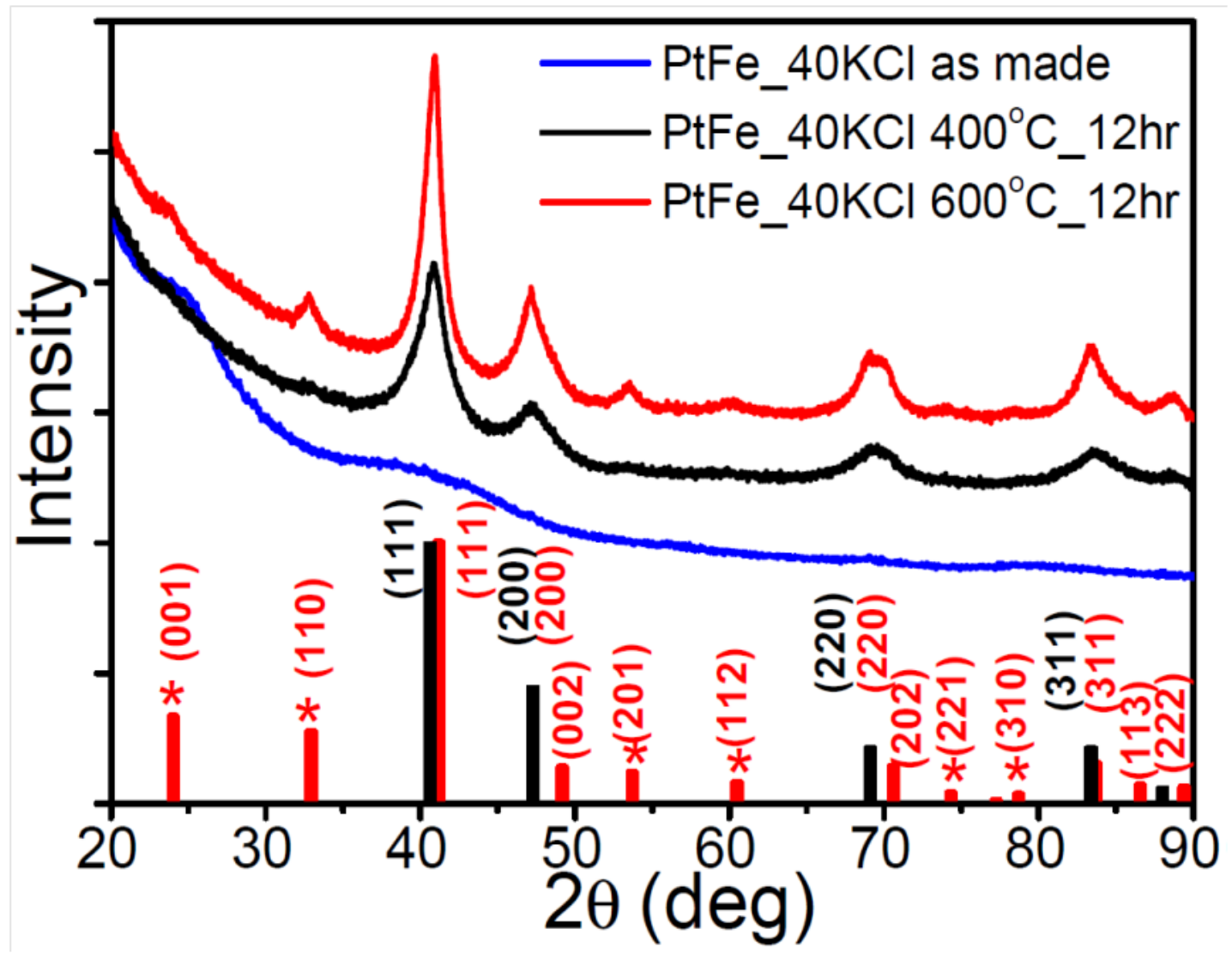

Figure 3.17: XRD patterns of PtFe from a PtFe-40KCl matrix with no thermal annealing (blue) and annealed for 12 hours at $400{ }^{\circ} \mathrm{C}$ (black) and $600{ }^{\circ} \mathrm{C}$ (red), respectively. After annealing the $\mathrm{PtFe}-40 \mathrm{KCl}$ matrix for 12 hours, the $\mathrm{KCl}$ matrix is removed. The peak positions for the disordered alloy phase (PDF\#030659122) and intermetallic phase (PDF\# 030659121) of PtFe are shown at the bottom as black and red tick marks, respectively. The superlattice peaks from ordering are marked with *. 
As shown in the X-ray diffraction (XRD) pattern (figure 3.17), the as-synthesized PtFe nanoparticles are essentially amorphous or have very small domain sizes. After annealing at $400{ }^{\circ} \mathrm{C}$ for 12 hours under vacuum, the PtFe nanoparticles remain in an alloy FCC structure. However, after increasing the annealing temperature to $600{ }^{\circ} \mathrm{C}$ for 12 hours, the ordered tetragonal phase is obtained. The observation of ordering peaks, such as the expected weak (001), (110) and (201) peaks, demonstrates that the desired ordered intermetallic phase is formed. The average domain size and average particle size have increased to $5 \mathrm{~nm}$ (figure 3.18).

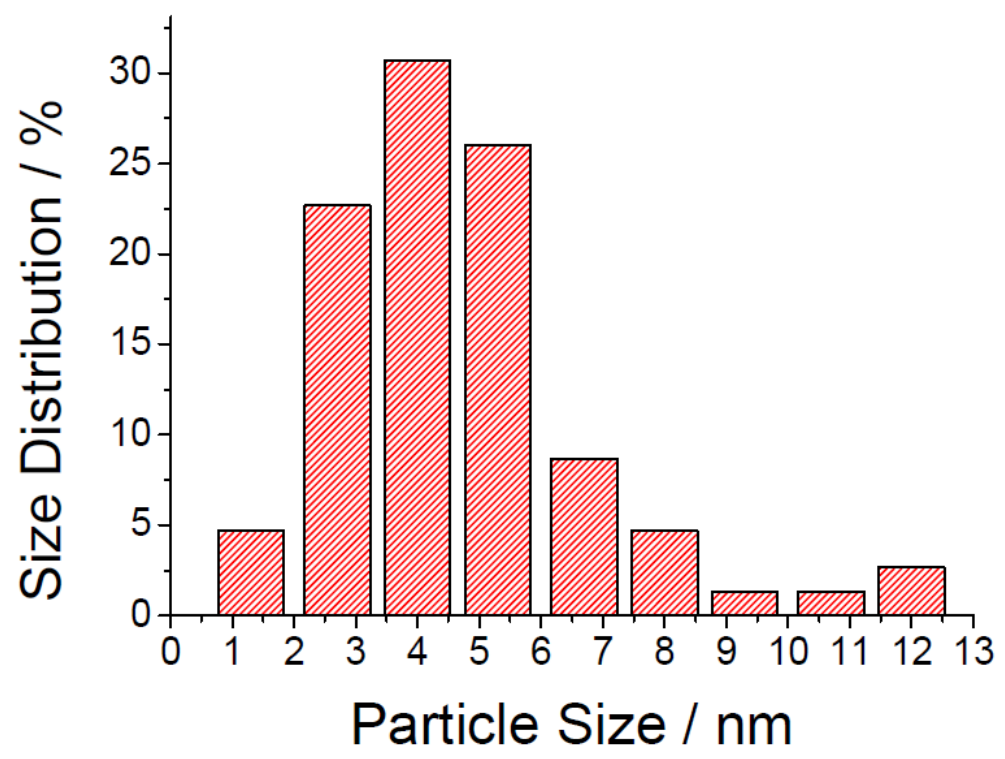

Figure 3.18: Size distribution of PtFe- $40 \mathrm{KCl}$ annealed at $600{ }^{\circ} \mathrm{C}$ for $12 \mathrm{hr}$. More than a hundred and fifty nanoparticles were analyzed and which shows the average nanoparticle size is $5 \mathrm{~nm}$. 
With comparable domain and particle sizes, there are few internal grain boundaries and their area is smaller than that of the external surfaces. Grain boundary planes are expected to become more important as the sintering becomes more pronounced, but for the annealing conditions identified here these effects are still quite small. The regime studied here would be best described as the initial stages of sintering, as there are many fewer grain boundary planes than external surfaces.

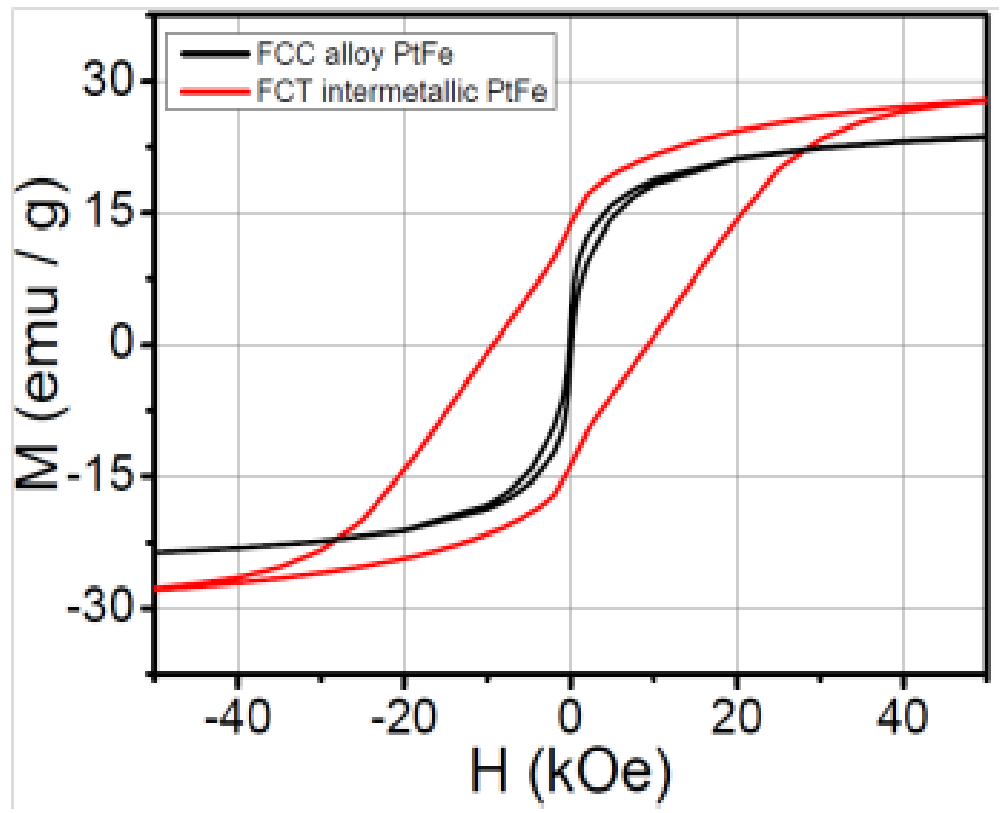

Figure 3.19: SQUID data of hysteresis loops of the PtFe nanoparticles obtained after the thermal annealing of the PtFe-40KCl complex at $400{ }^{\circ} \mathrm{C}$ (black) and $600{ }^{\circ} \mathrm{C}$ (red) for 12 hours under vacuum. 
In contrast to PtFe alloys, the atomically-ordered intermetallic structure is ferromagnetic and shows large uniaxial magnetocrystalline anisotropy and good chemical stability ${ }^{11}$ Magnetic measurements at room temperature of the PtFe-40KCl (mole ratio of $\mathrm{PtFe}$ to $\mathrm{KCl}$ matrix is $1: 40$ ) annealed at $400{ }^{\circ} \mathrm{C}$, taken in a Quantum Design superconducting quantum interference device (SQUID - Model Quantum Design MPMS-XL7), exhibit superparamagnetic behavior (figure 3.19). In summary, XRD and magnetic data clearly show that annealing at $400{ }^{\circ} \mathrm{C}$ for 12 hours did not convert the FCC PtFe to the ordered intermetallic tetragonal structure. In contrast, $\mathrm{PtFe}-40 \mathrm{KCl}$ annealed at $600{ }^{\circ} \mathrm{C}$ for 12 hours is ferromagnetic with a coercivity of almost $1 \mathrm{~T}$ at $300 \mathrm{~K}$. A saturation magnetization of 29 emu per gram of $\mathrm{PtFe}$ is observed (figure 3.19). In comparison, the saturation magnetization of Fe metal is 220 emu per gram. The smaller moment of PtFe is due, in part, to the small particle sizes, the dilution of $\mathrm{Fe}$ by nonmagnetic $\mathrm{Pt}$, and the much larger mass of Pt related to $\mathrm{Fe}$. Previous authors have reported values as high as $56 \mathrm{emu}$ per gram for $7 \mathrm{~nm}$ diameter PtFe particles. ${ }^{11}$ Nevertheless, such contrast in their magnetic properties also demonstrates that the ordered phase is clearly achieved.

After thermal annealing a PtFe- $40 \mathrm{KCl}$ sample at $600{ }^{\circ} \mathrm{C}$ for 12 hours to achieve the ordered intermetallic phase, the $\mathrm{KCl}$ matrix was removed and atomic-resolution highangle annular dark field (HAADF) STEM images of the annealed nanoparticles were taken. As shown in figure 3.20a-c, due to the atomic-number ' $\mathrm{Z}$ contrast' of the HAADF signal, the Pt shows up as much brighter than the Fe signal. The STEM images show periodic changes of brighter and darker columns of atoms, which suggests the ordering of the two elements in the nanoparticles, indicating the presence 
of the ordering structure on the zone axis observed. ${ }^{30}$

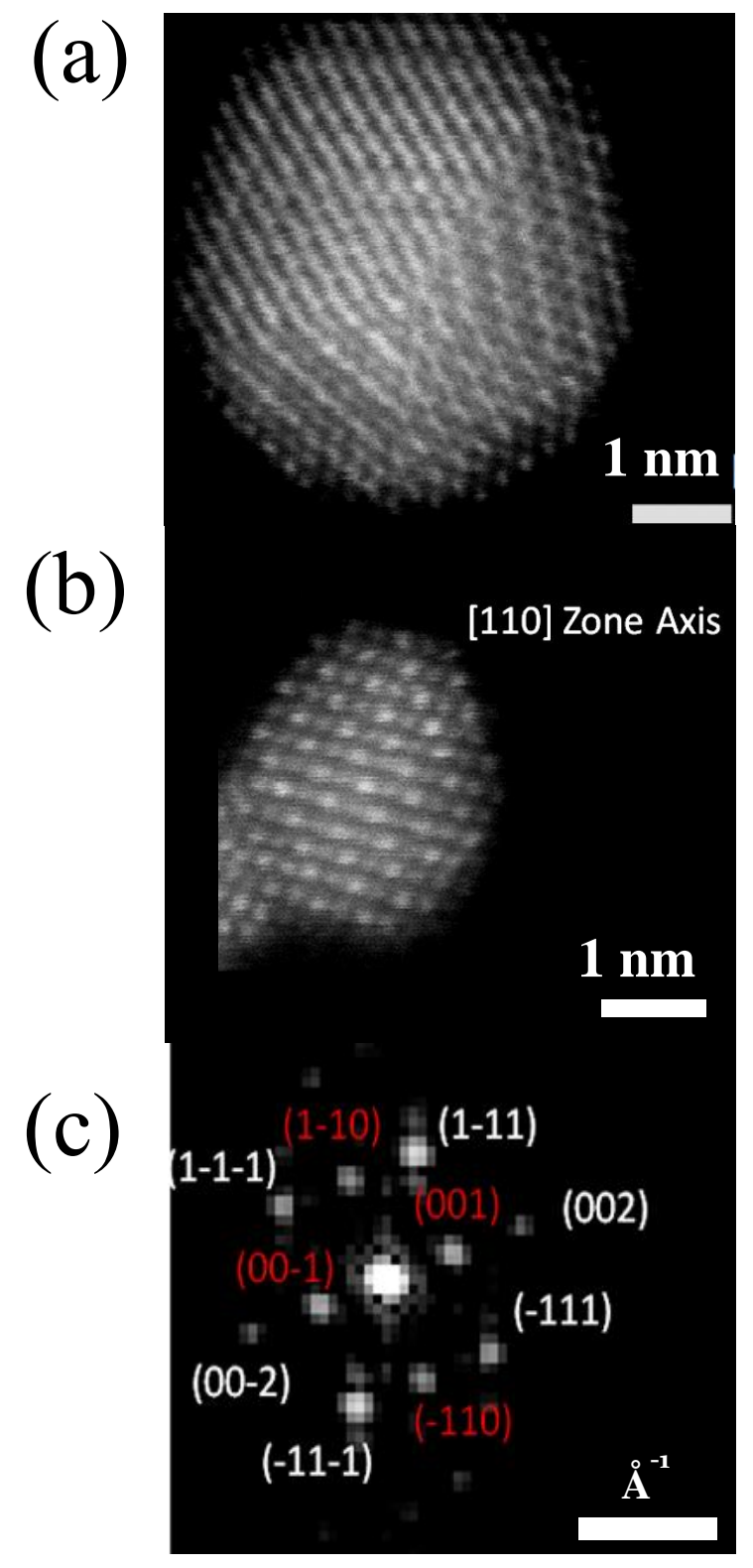

Figure 3.20 (a)-(c) HAADF-STEM images of the annealed intermetallic nanoparticles.

(b) The ordered intermetallic structures viewed on [110] zone axis, where periodic arrays of brighter and darker atoms present Pt and Fe, respectively. (c) Diffractogram of (b) showing superlattice peaks such as the $\{001\}$ and $\{110\}$ marked with red. 
As shown in figure $3.20 \mathrm{~b}$, an intermetallic nanoparticle is observed on the [110] zone axis. The intermetallic structure can also be realized by the appearance of the superlattice planes in the electron diffraction pattern of a single crystal domain. Shown in figure $2 \mathrm{c}$ is the diffractogram from figure $2 \mathrm{~d}$ with the superlattice spacings marked in red. Spacings of $3.7 \AA$ and $2.7 \AA$ are measured for the $\{001\}$ and $\{110\}$ superlattice spots, respectively, and matches with the bulk PtFe intermetallic structure. These data confirm the formation of an ordered intermetallic phase after thermal annealing at 600 ${ }^{\mathrm{o}} \mathrm{C}$.
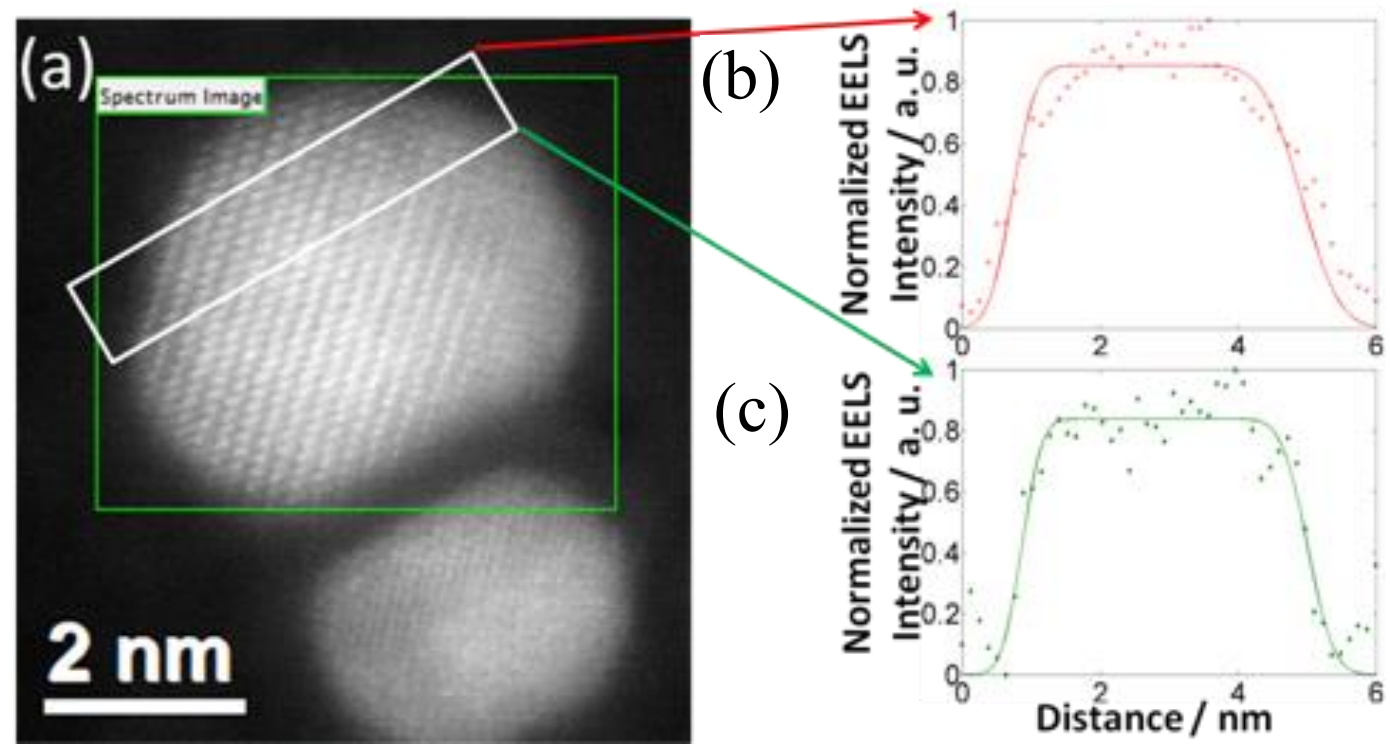

Figure 3.21: (a) HAADF-STEM image. The line profiles of (b) Fe and (c) Pt show homogenous distributions of Fe and Pt, without any obvious core-shell structure, within the experimental resolution of less than one atomic layer. The best-fit lines are extrapolated by using two error functions and the distance is calculated based on the full width at half maximum of the best-fit line. 
We have also determined the composition of individual annealed nanoparticles by performing electron-energy loss spectroscopy (EELS) imaging. An on-axis intermetallic nanoparticle is shown in figure 3.21a. The Fe signal is extracted using a power-law background fit on the $\mathrm{Fe}-\mathrm{L}_{2,3}$ edge, since the peaks have a distinct and abrupt onset. However, since the broad Pt-M and Pt-N edges have delayed onsets, we used the MCR (multivariate curve resolution) method to extract the Pt- $\mathrm{N}_{3}$ edge, although background subtraction still works well for the higher-energy Pt-M edge. Details and tests of the reliability of this approach for analyzing the Pt- $\mathrm{N}_{3}$ edge can be found in previous publications ${ }^{31,32}$ and their supplements. The EELS study suggested a homogenous distribution of $\mathrm{Fe}$ and $\mathrm{Pt}$, with no obvious phase segregation within the experimental precision of less than one atomic layer (figure 3.22a-c). To quantitatively evaluate whether a core-shell structure exists, EELS line profiles were obtained (figure $3.21 \mathrm{~b}$ and $\mathrm{c}$ ), and the full width at half maximum (FWHM) of the projected $\mathrm{Fe}$ and $\mathrm{Pt}$ mass profiles were extracted. The difference between the FWHM of the Pt and Fe signals is less than one atomic layer, indicating no obvious core-shell formation. Such homogeneity is also expected since the thermal annealing was performed under vacuum rather than in a reducing environment, such as hydrogen. Under a hydrogen atmosphere, Pt usually segregates to the surface in order to lower the surface energy through the formation of relatively strong Pt-H bonds. ${ }^{33}$ 


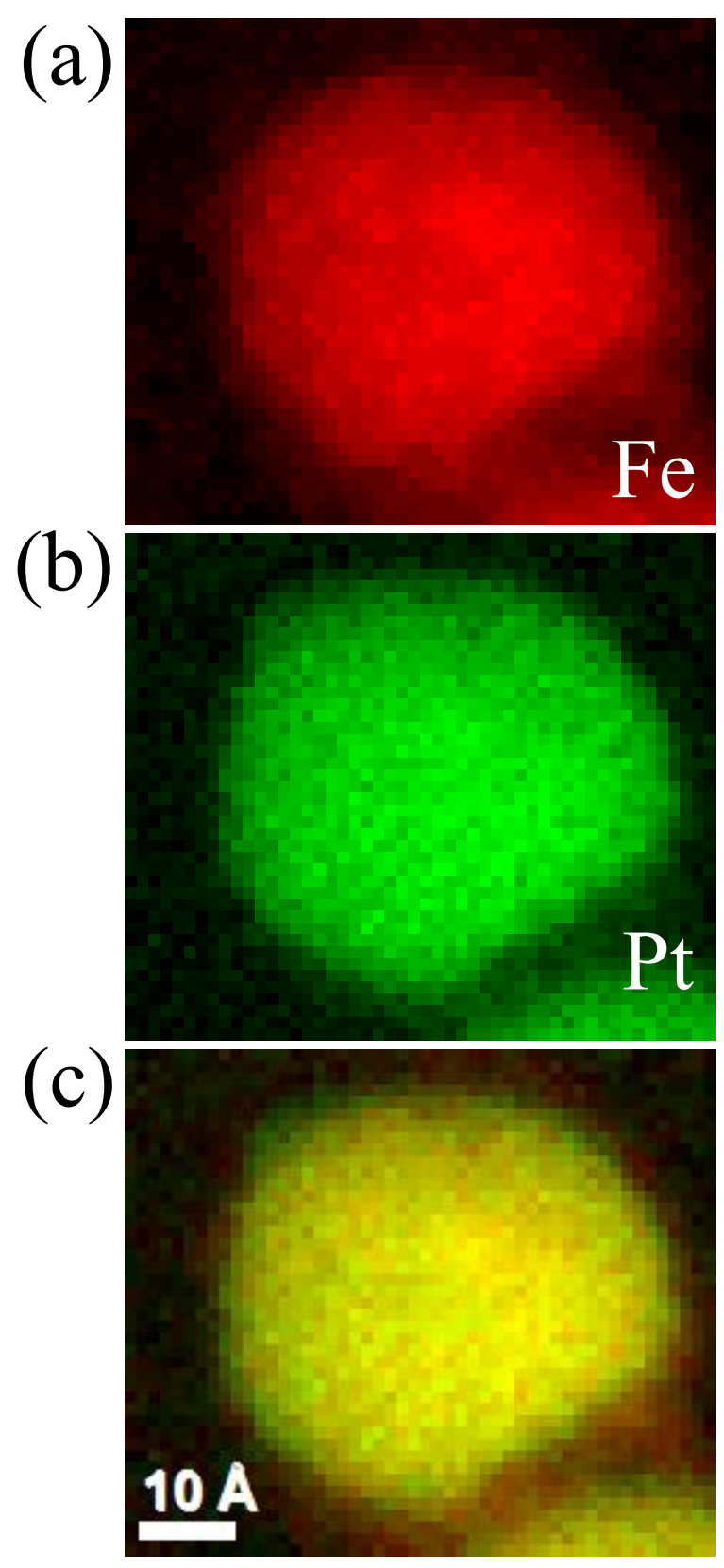

Figure 3.22: EELS spectrum images shown in (a) Fe- $\mathrm{L}_{2,3}$ edge, extracted by powerlaw background fit; (b) Pt- $\mathrm{N}_{3}$ edge, extracted after 20 iterations of MCR (multivariate curve resolution) and (c) composite of Fe and $\mathrm{Pt}$. 


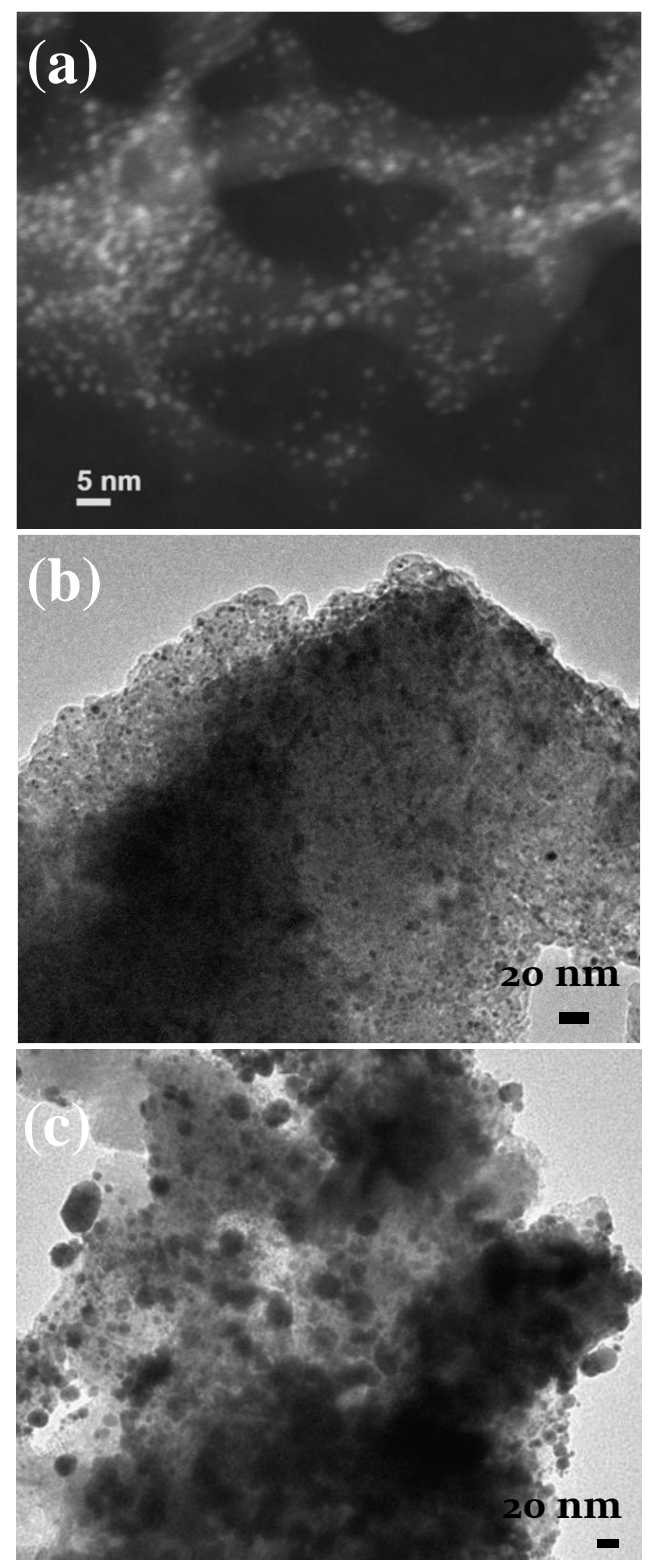

Figure 3.23: (a) STEM image of as prepared disordered FCC PtFe nanoparticles with $\mathrm{KCl}$ matrix. (b) TEM image of $\mathrm{PtFe}-40 \mathrm{KCl}$ annealed at $600{ }^{\circ} \mathrm{C}$ for 12 hours. (c) TEM image of $\mathrm{PtFe}-7 \mathrm{KCl}$ (Mole ratio of $\mathrm{PtFe}$ to $\mathrm{KCl}$ is 7) annealed at $600{ }^{\circ} \mathrm{C}$ for 12 hours. This sample is synthesized according to: $\mathrm{PtCl}_{4}+\mathrm{FeCl}_{3}+7 \mathrm{KEt}_{3} \mathrm{BH} \rightarrow \mathrm{PtFe}+7 \mathrm{KCl}+$ $3.5 \mathrm{H}_{2}+7 \mathrm{Et}_{3} \mathrm{~B}$. As a comparison to (b), with less $\mathrm{KCl}$ salt, particle sizes range from more than $40 \mathrm{~nm}$ to $2 \mathrm{~nm}$, which is a result of significant particle aggregation. 
The STEM images of the as-prepared disordered FCC and ordered tetragonal PtFe nanoparticles in the $\mathrm{KCl}$ matrix are shown in figure 3.23. The as-prepared $\mathrm{PtFe}$ nanoparticles average less than $2 \mathrm{~nm}$ in diameter. Even after thermal annealing at 600 ${ }^{\circ} \mathrm{C}$ for 12 hours, the $\mathrm{KCl}$ matrix protects the nanoparticles from significant large-scale aggregation (figure $3.23 \mathrm{~b}$ and c). The final particle sizes are controlled at around 4 to 5 nm on average (figure 3.18).

3.3.3 The particle growth mechanism during thermal annealing of the $\mathrm{Np}-\mathrm{KCl}$

To fully understand the particle growth process, an in-situ STEM annealing study of the $\mathrm{Np}-\mathrm{KCl}$ assembly on a heated substrate was undertaken. ${ }^{34,35}$ Since the nanoparticles are formed in or on the $\mathrm{KCl}$, the $\mathrm{Np}-\mathrm{KCl}$ assembly was transferred onto the heating grid in an Ar-protected atmosphere, preventing any interference from air. During imaging, the sample was heated to $400{ }^{\circ} \mathrm{C}$ and then to $500{ }^{\circ} \mathrm{C}$, holding for c.a. 20 minutes at each temperature. The temperature was then increased to $600{ }^{\circ} \mathrm{C}$ and held there for two hours (figure 3.24). Nanoparticle sintering was observed during the heat treatment.

In general, there are two competing mechanisms in the sintering process, namely Ostwald ripening and coalescence. ${ }^{36,37}$ Ostwald ripening is caused by the exchange of atoms, one at a time, with larger particles growing and smaller particles continuously shrinking in size. Coalescence is the result of the motion of the nanoparticles themselves. 


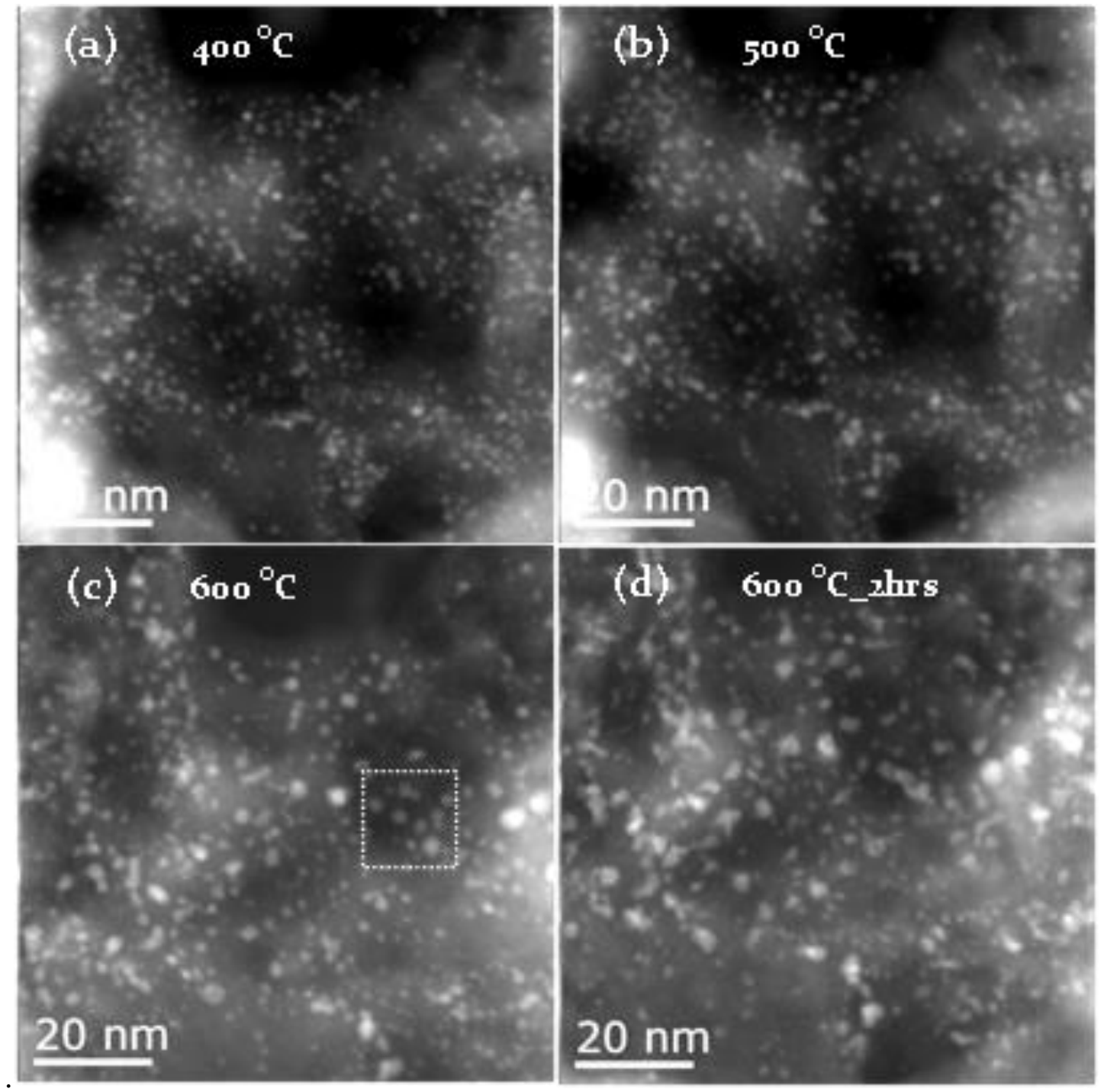

Figure 3.24: HAADF-STEM images of one representative area during the in-situ heating experiment. (a) $400^{\circ} \mathrm{C}$; (b) $500^{\circ} \mathrm{C}$; (c) $600^{\circ} \mathrm{C}$; (d) $600^{\circ} \mathrm{C}$ and after 2 hours; 
In Ostwald ripening, the larger particles grow at the expense of the smaller ones; hence, as the size distribution evolves, it is expected to have a bias toward larger diameters. However, such distributions can only be observed if sufficient time is allowed for the particles to grow to about 10 times their initial diameter, such that any history of the initial distribution is lost. ${ }^{38,39}$ Coalescence, in contrast, is the merging of two nanoparticles. Studies have suggested that a log-normal distribution should result from coalescence. ${ }^{40-43}$

To determine the dominant growth mechanism, we followed the time evolution of the particle positions and sizes over the same area of the $\mathrm{PeFe}-\mathrm{KCl}$ assembly under thermal annealing up to $600{ }^{\circ} \mathrm{C}$ (figure 3.24a-d). We found that most coarsening events occurred primarily through the motion of the nanoparticles in or on the $\mathrm{KCl}$ matrix, which suggests that coalescence is the main coarsening mechanism. In addition, the particle size distribution of over four hundred nanoparticles in the same area, at different temperatures, was measured in-situ (figure 3.25a). As the temperature increased, the mean diameter of the particles became larger, along with a broadening of the size distribution. ${ }^{40-43}$ The size distribution is plotted in figure $3.25 \mathrm{~b}$, and fits well to the log-normal distribution that is consistent with coalescence. ${ }^{43-46}$ To illustrate the coalescence process, the evolution of several particles was shown in figure 3.26. Nanoparticles, instead of atoms, diffuse in or on the $\mathrm{KCl}$ matrix and coalesce to form bigger particles. 
(a)
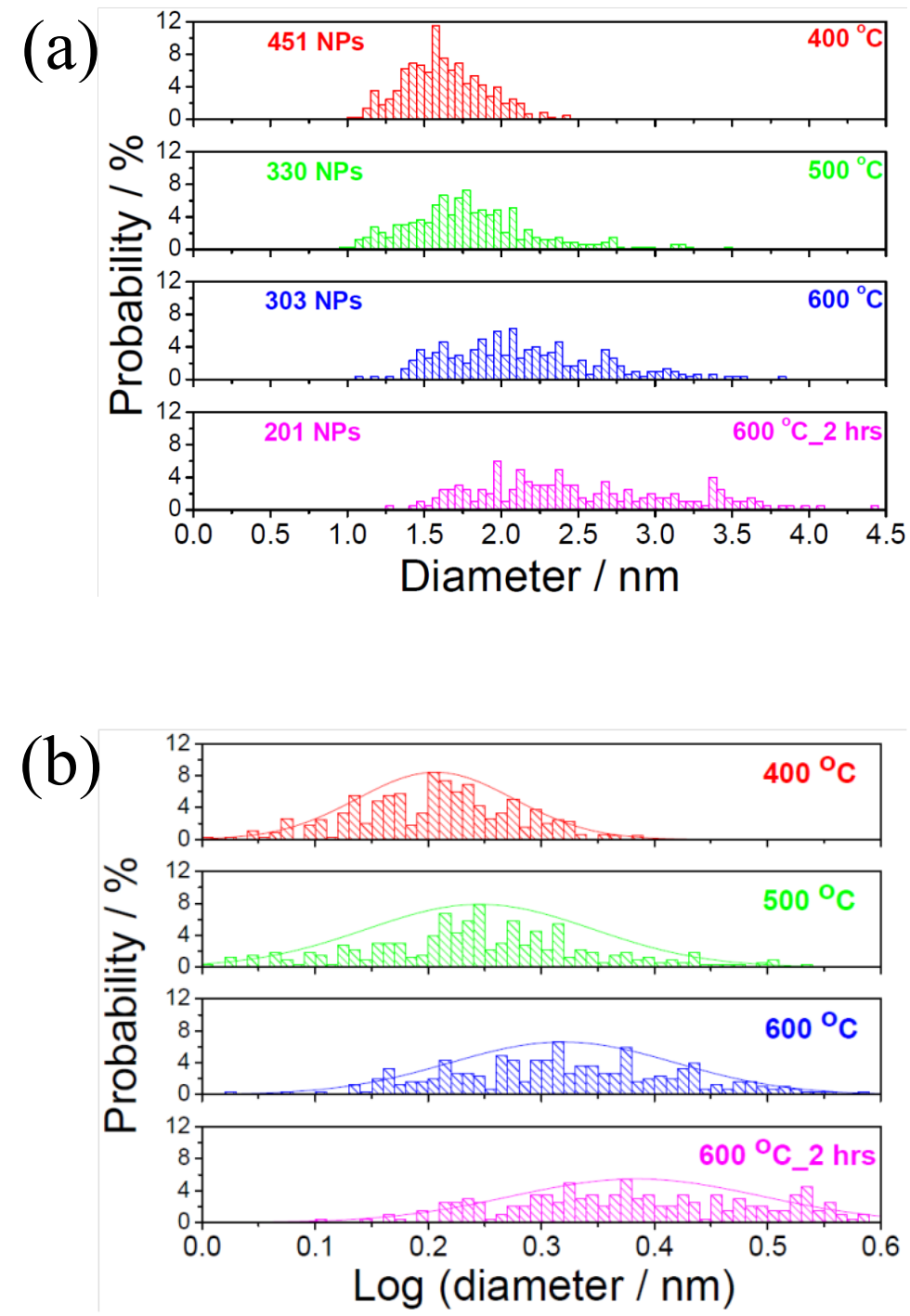

Figure 3.25: (a) Statistical histogram of size distribution in figure 3.24 (a)-(d); (b) Log scale of size distribution, fitted with a log-normal distribution. The maintenance of such a log-normal distribution is consistent with coalescence. 


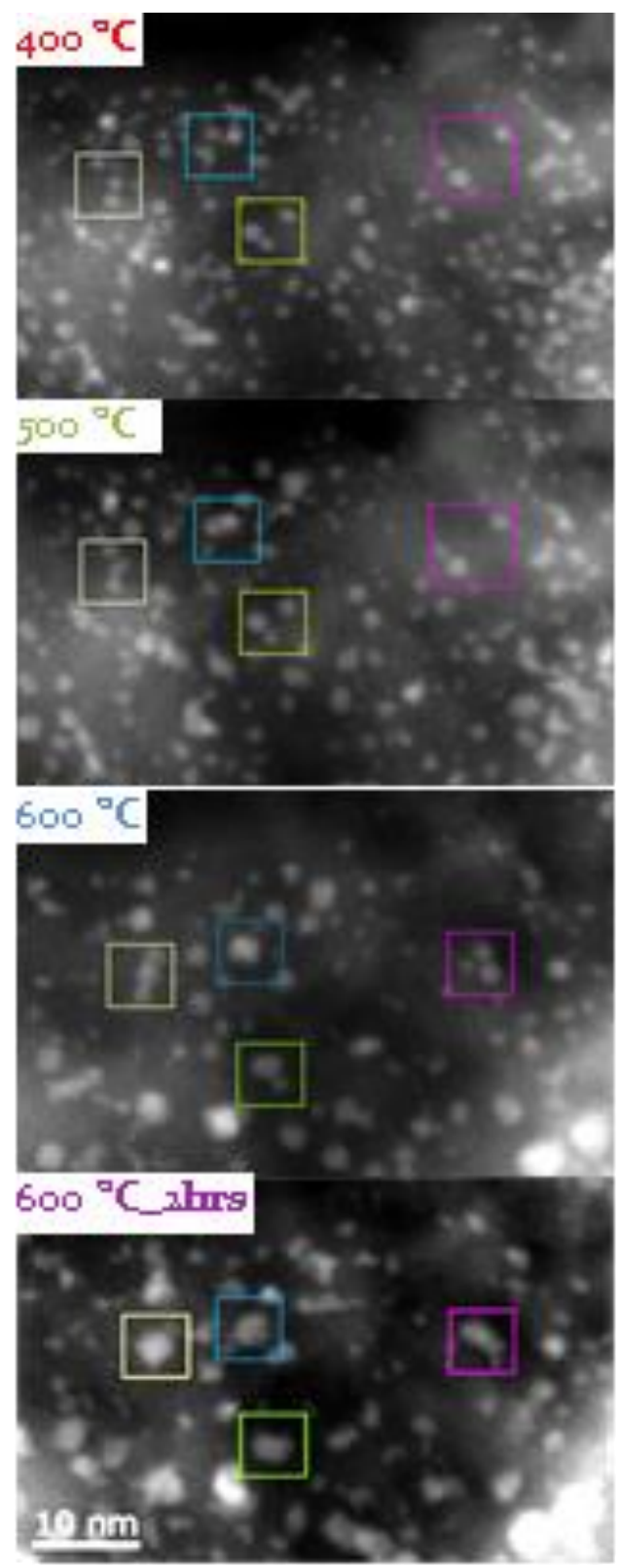

Figure 3.26: Zoomed in images of figure 3.24 (a)-(d). The evolution of four particles has been tracked with changes in temperature, showing that coalescence is the main mechanism for particle growth. 
To evaluate the robustness of the particle size measurement, two independent measurements of two areas with different numbers of nanoparticles were conducted at each temperature frame and showed similar size distribution. As shown in figure 3.27, the shapes of histograms from the two data sets match each other, indicating good precision of the particle size measurement at the areas analyzed.
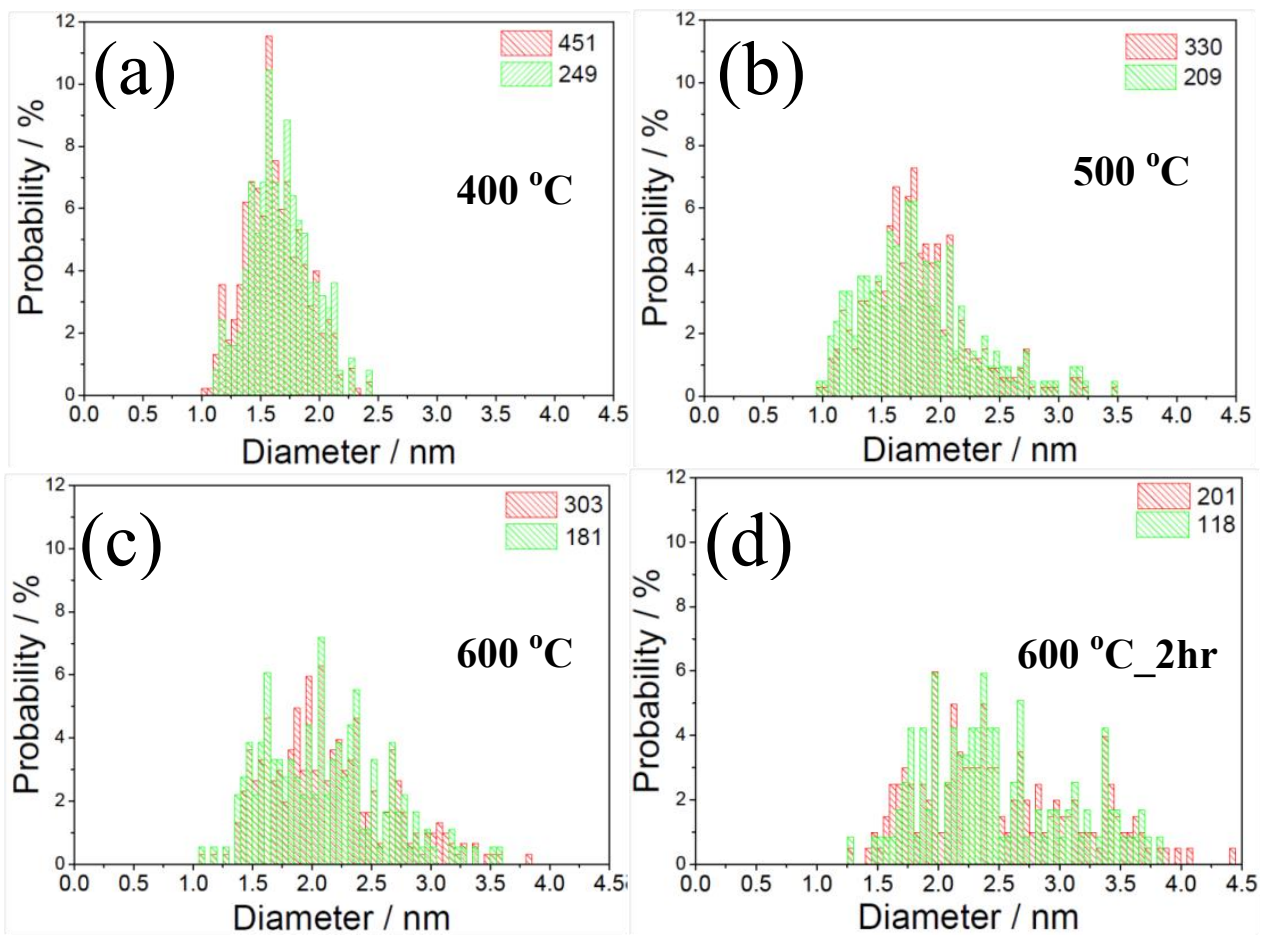

Figure 3.27: Two independent measurements of areas with different numbers of nanoparticles were conducted at each temperature frame. The two sets of particle size statistics were conducted at (a) $400{ }^{\circ} \mathrm{C}$, (b) $500{ }^{\circ} \mathrm{C}$, (c) $600{ }^{\circ} \mathrm{C}$, (d) $600{ }^{\circ} \mathrm{C}$ after 2 hours annealing, and are denoted by red and green in the figures. The shapes of histograms from the two data sets match each other, indicating good precision of the particle size measurement at the areas analyzed. 
Although the size distribution is consistent with the existence of coalescence, the size distribution alone is not sufficient to eliminate the occurrence of Ostwald ripening, whose ideal distribution will not be apparent unless the average diameter is increased by a factor of 10 . To address this, we tracked the size and positions of individual particles in a selected area during a more detailed time series, instead of relying solely on the size distribution.

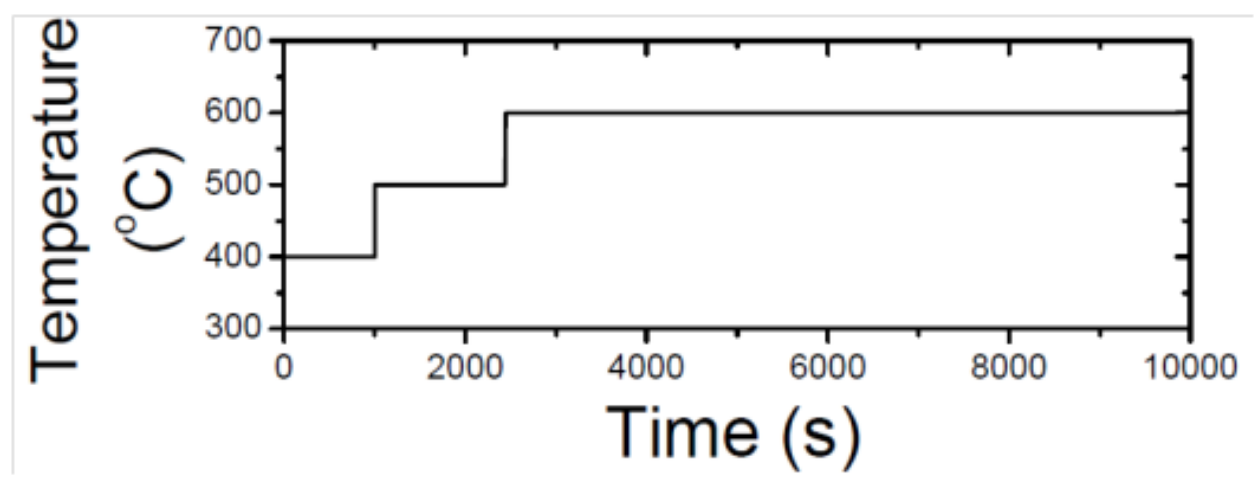

Figure 3.28: Plot of grid temperature versus real time during the in-situ heating experiment. The sample was heated to $600{ }^{\circ} \mathrm{C}$ at $2442 \mathrm{~s}$ and kept at that temperature for two hours. An isolated area, marked in figure 3.24(c), has been imaged every 3 minutes to track the change of particle sizes.

Figure 3.28 presents a plot of the grid temperature versus real time. The area shown in figure $3.24 \mathrm{c}$ was imaged every 3 minutes at $600{ }^{\circ} \mathrm{C}$ for two hours. In a region of 150 
nanoparticles, 22 instances of coalescence versus 4 instances of Ostwald ripening were counted. As an example, a selected area, which is circled in figure $3.24 \mathrm{c}$, is shown in figure 3.29. In the initial stages of coalescence, the particles are not all round or oval in shape.

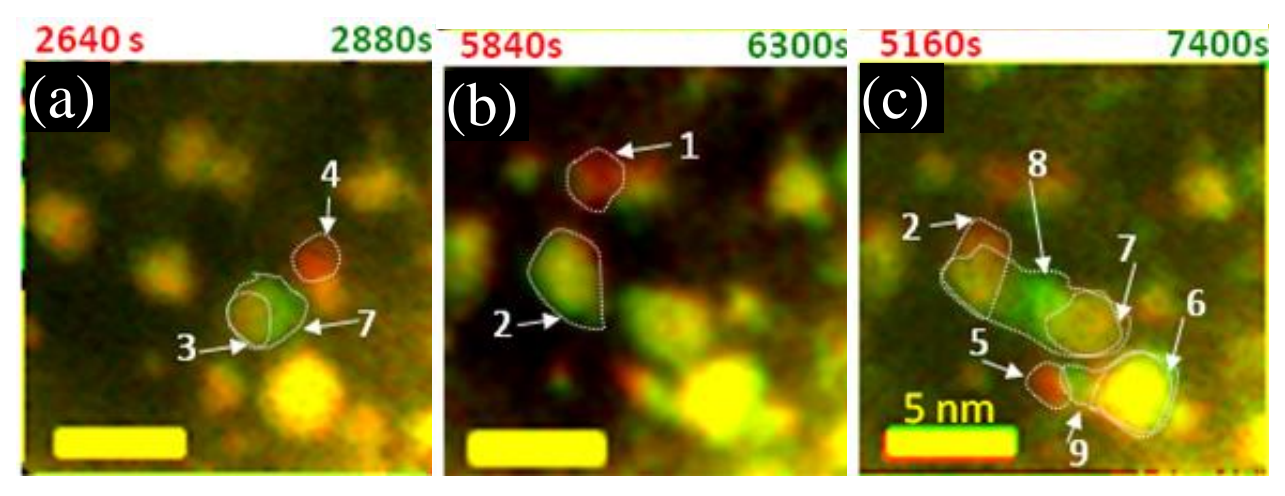

Figure 3.29: Video images of corresponding particles in the selected area in figure $3.24 \mathrm{c}$ showing the particle growth pathways. The images are colored with red (for before i.e. t-1) and green (for after, i.e. t) corresponding to different time frames labeled on the top. If the particles did not move, a yellow color is expected. If the particles disappeared from one location, a red color is expected at the original location.

If the particles migrated or are newly formed, a green color is expected at the new location. Ostwald ripening occurred in (a) from particles \# 1 to \# 2. Coalescence took place in (b) with the formation of particle \# 7 from \# 3 and \# 4. Two other cases of coalescence can be seen in (c) where the formation of \# 9 is from the migration and sintering of \# 5 and \# 6, and formation of \# 8 is from \# 2 and \# 7. In 150 total particles, 22 instances of coalescence and only 4 instances of Ostwald ripening were observed. 
To assist the reading, snap shots of the images in figure 3.29 without overlapping and with all particles labeled are shown in figure 3.30.

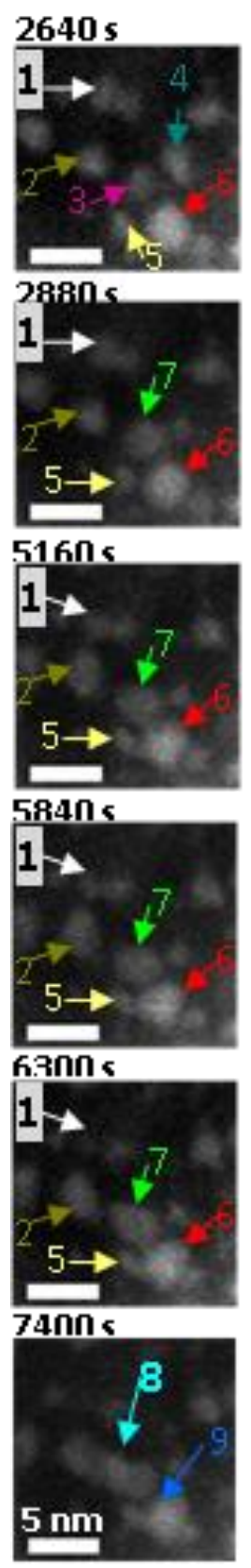

Figure 3.30: the images in figure 3.29 without overlapping and with all particles labeled at different time frames are shown. 

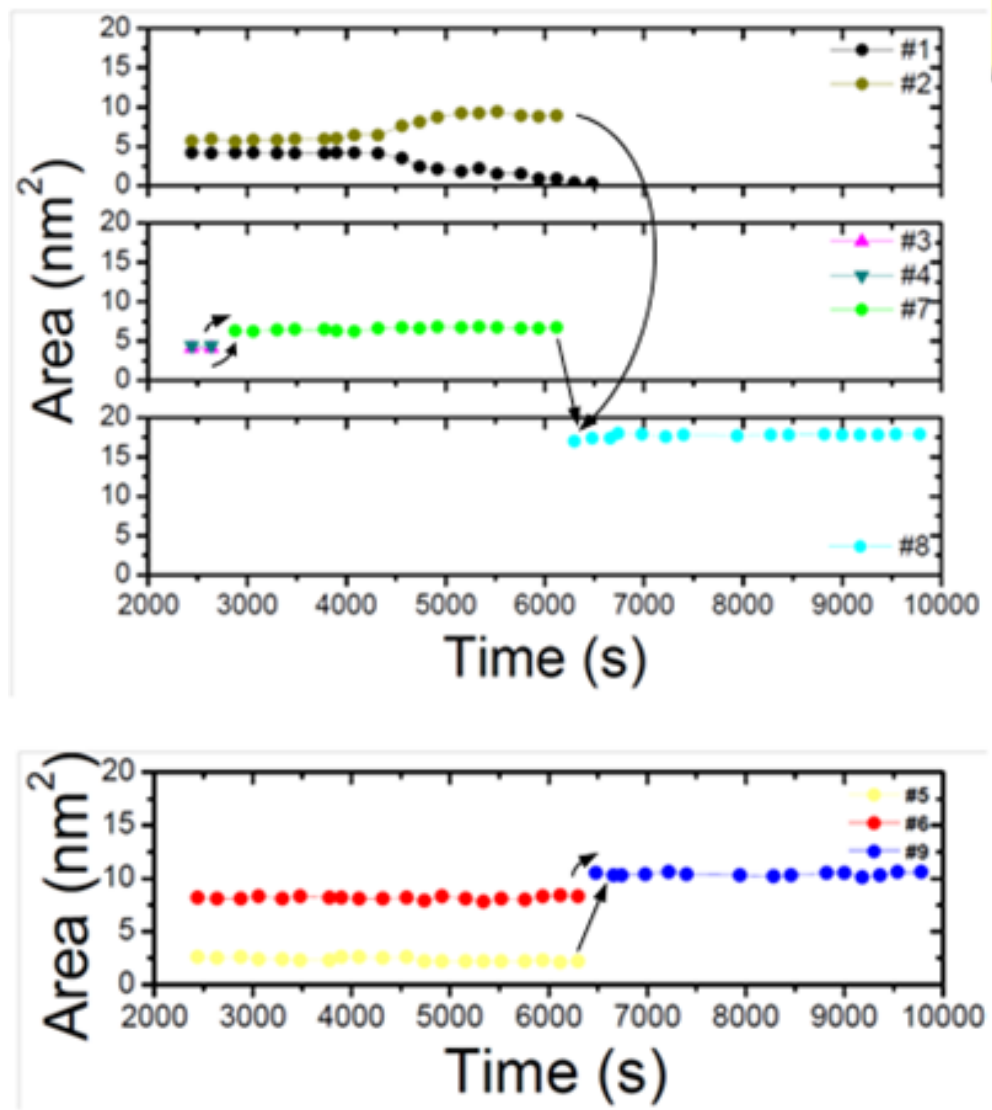

Figure 3.31: Particle sizes versus real time corresponding to the video images in figure 3.29. To better illustrate the subtle changes in particle sizes, we have segmented the nanoparticles with polygons, which are also for area estimation. A detailed example is shown in figure 3.31 . 


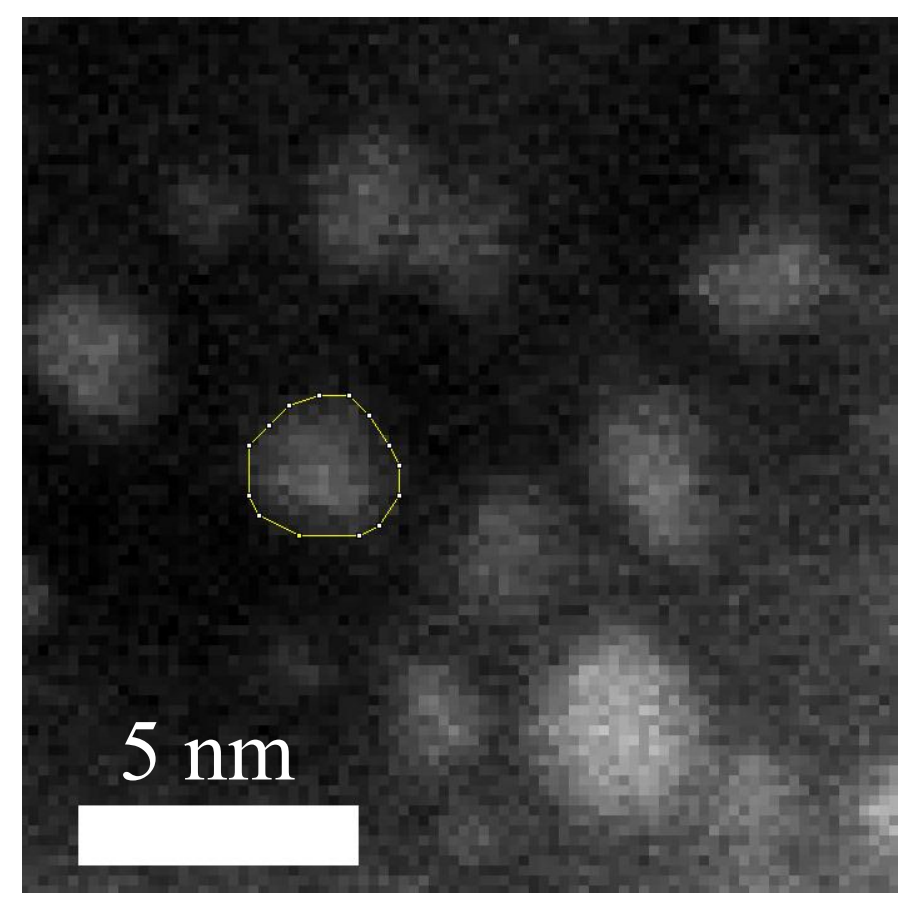

Figure 3.32: Especially at the beginning stage of coalescence, particles are not all round, or oval shape. In figure 5, to better illustrate the subtle changes in particle sizes, we have outlined the edges of particles based on the intensity contrast of particles (brighter) and background (darker) from the STEM image. The areas of the outlined 2D projection of nanoparticles were then used to represent particle sizes. Each particle is contoured manually and the selected area is calculated by image J. This method of determining particle size is more realistic than using best fit circles to represent the particle area. 


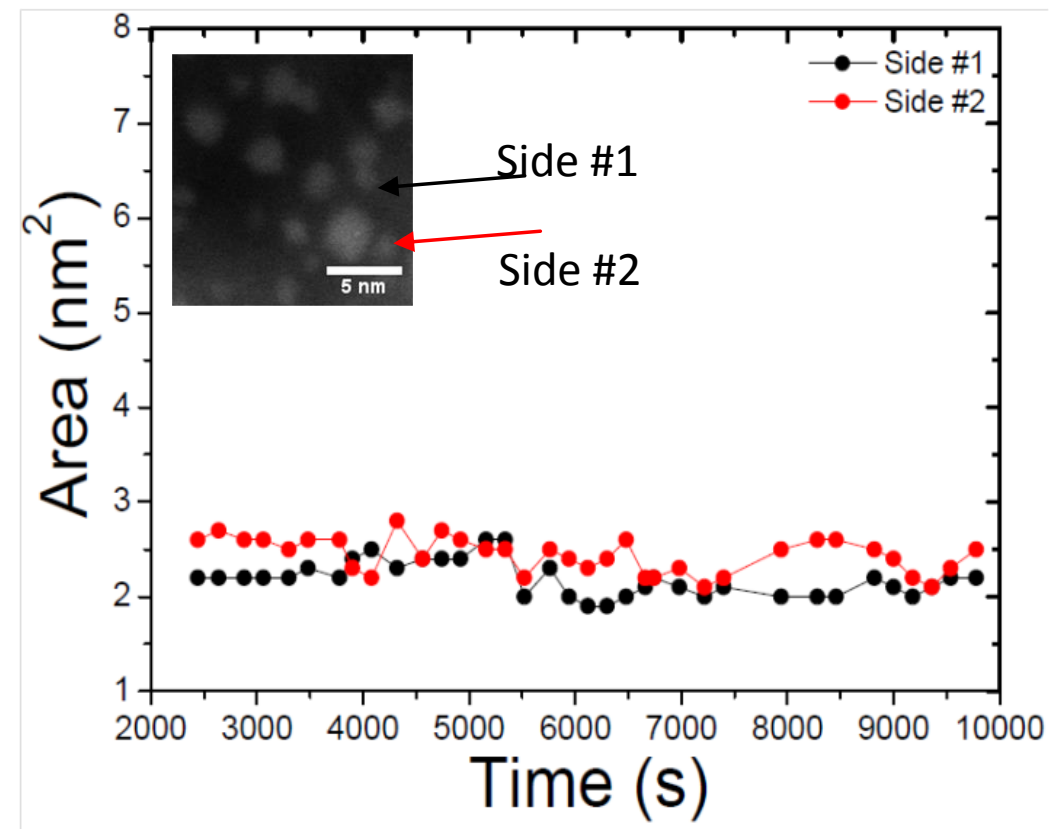

Figure 3.33: The sizes of particles closest to the target particles in figure 3.29 were measured and showed no significant change.

To better illustrate the subtle changes in particle sizes, we measured the projected areas of the nanoparticles to represent the particle sizes. This assumes that the third, projected dimension, does not deviate significantly in scale from the other two dimensions (A more detailed method is included in figure 3.32). To eliminate the influence of particles that are outside the observed area, the sizes of particles closest to the target particles were measured and showed no significant changes (figure 3.33). The particle sizes in the observed area are shown in figures 3.31. Shown in figure 3.29 are the images of the selected area at specific times. The corresponding particles are 
marked in the images. Coalescence of particles was observed and dominated the particle growth process. Note the formation of particle \#7 from particles \#3 and \#4 at $2880 \mathrm{~s}$, the formation of particle \#8 from particle \#7 and \#2 at $7400 \mathrm{~s}$, and the formation of particle \#9 from \#5 and \#6 at 7400 s. A common pattern has been observed for these coalescence events, in which two particles diffuse toward each other and subsequently merge. Ostwald ripening was also observed in a few instances as well. The gradual size increase of particle \#2 and size decrease of particle \#1 evidence a clear pattern for ripening. As mentioned previously, there were very few Ostwald ripening events (4 incidences out of 150 particles) compared to coalescence events (22 incidences out of 150 particles).

The different shapes of nanoparticles are also an indication of the sintering mechanism. When Ostwald ripening occurs, as for particles \#1 and \#2 in figure 3.29, the shapes before and after are closer to round or oval. This can be understood in terms of the exchange of individual atoms between particles. Individual atoms, that attach to each particle, are more likely to locate at sites that minimize the overall surface/volume ratio (which, in turn, minimizes the surface energy), while particles are most likely to detach from sites that maximize overall surface/volume ratio. The end result is a tendency toward more rounded particles. This process is isotropic in nature and thus should not lead to any preferential shape. However, this is not the case for coalescence. The joining of two or more nanoparticles will occur when the two particles first touch, initially leading to elongated structures. If the particles have different orientations, then a grain boundary will be created where the particles touched. After significant surface diffusion, a rounder shape evolves to lower the 
energy of the system. As an example, in figure 3.26, several particles that display this elongated shape are highlighted. However, like other TEM/STEM studies, this in-situ STEM study is based on a $2 \mathrm{D}$ projection of $\mathrm{Np}-\mathrm{KCl}$ matrix during thermal annealing. Based solely on one projected image, it is almost impossible to distinguish if the two nanoparticles overlap or sinter. However, in this study, since the in-situ observation recorded contains multiple snapshots at different temperatures and times, it is sometimes possible to distinguish particle overlap from sintering by their relative motions. One example can be seen in figure 3.29a, where the yellow colored nanoparticle below \# 4 can be categorized as an overlapping rather than a sintered one.

As a short summary, the in-situ STEM studies allowed the tracking of growth and motion of individual particles during thermal annealing of the $\mathrm{Np}-\mathrm{KCl}$ assemblies. This study shows that encasing nanoparticles in a $\mathrm{KCl}$ matrix significantly slows agglomeration and particle size growth. Based on these observations, we conclude that coalescence is the dominant mechanism of nanoparticle growth in the $\mathrm{KCl}$ matrix at the temperatures studied.

\subsection{To optimize the Np-KCl method}

As discussed above, the coalescence of the particles dominates the growing process. Therefore, the distribution of the as-reduced nanoparticles in the $\mathrm{KCl}$ matrix is critical to the size distribution after thermal annealing. A more homogenous distribution may provide more uniform collision of particles and therefore result in a narrower distribution of particle sizes after thermal annealing. However, the distribution of as- 
synthesized $\mathrm{Pt}_{3} \mathrm{Fe}$ or the $\mathrm{PtFe}$ nanoparticles at $\mathrm{KCl}$ is not uniform (figure 3.6 and figure 3.23a), which results in the broad size distribution of the nanoparticles after annealing (figure. 3.11a and figure 3.18). Significant portions of the particles are out of the 3 to $5 \mathrm{~nm}$ range. Hence, to get a narrower size distribution, the homogeneity of the as-reduced $\mathrm{Np}-\mathrm{KCl}$ assembly using our current synthesis technique, in which a reducing agent is introduced by syringe injection to a vigorously stirring metal precursor solution, needs to be improved. As a result, to achieve a narrower size distribution of post-annealed nanoparticles, a method with a more controllable and homogenous mixing during reduction is required.

As part of the future work, I propose the use of the microfluidic solution, where a fast and homogenous mixing of the reducing agent and the metal precursor solution could be provided ${ }^{47}$.

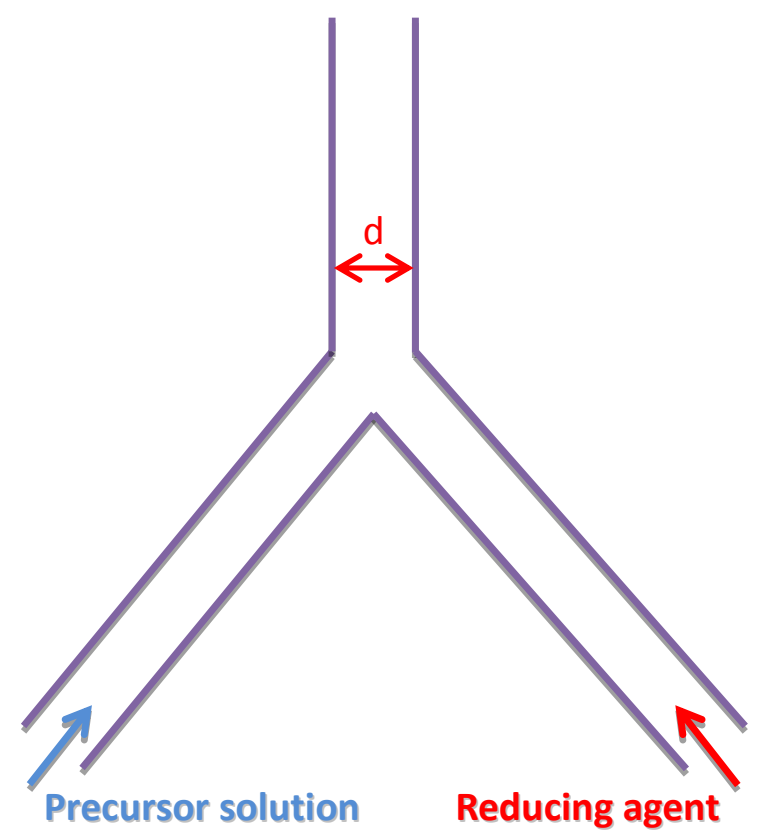

Figure 3.34: First design of the microfluidic device for the synthesis. 
Shown in figure 3.34 is the first design of the microfluidic device for the synthesis. The reducing agent and the precursor solutions are introduced through the two side channels. A homogenous and fast mixing will be achieved at the main channel with a diameter of d. Given that the diffusion coefficient for a common ion in aqueous solution is around $1 \sim 2 * 10^{-9} \mathrm{~m}^{2} \mathrm{~S}^{-1}$, the mixing time of $0.1 \mathrm{~S}$ will be achieved if the diameter of the channel is around $10 \mathrm{um}$. Such condition is suitable for current synthesis.
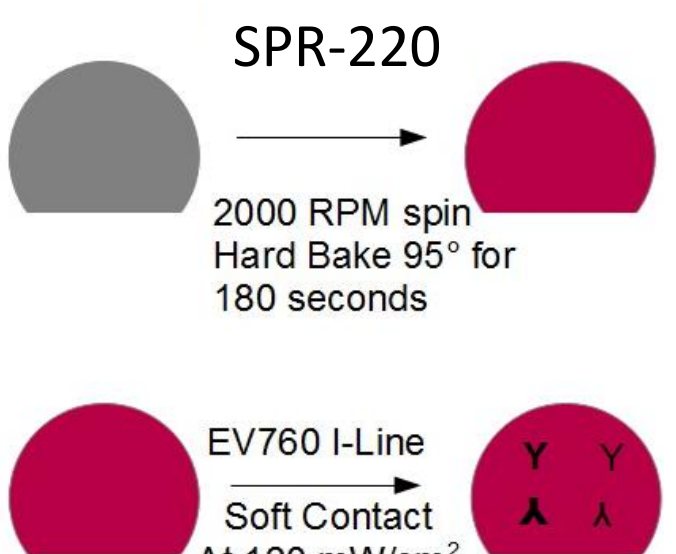
At $120 \mathrm{~mW} / \mathrm{cm}^{2}$ Developed with SR220 developer
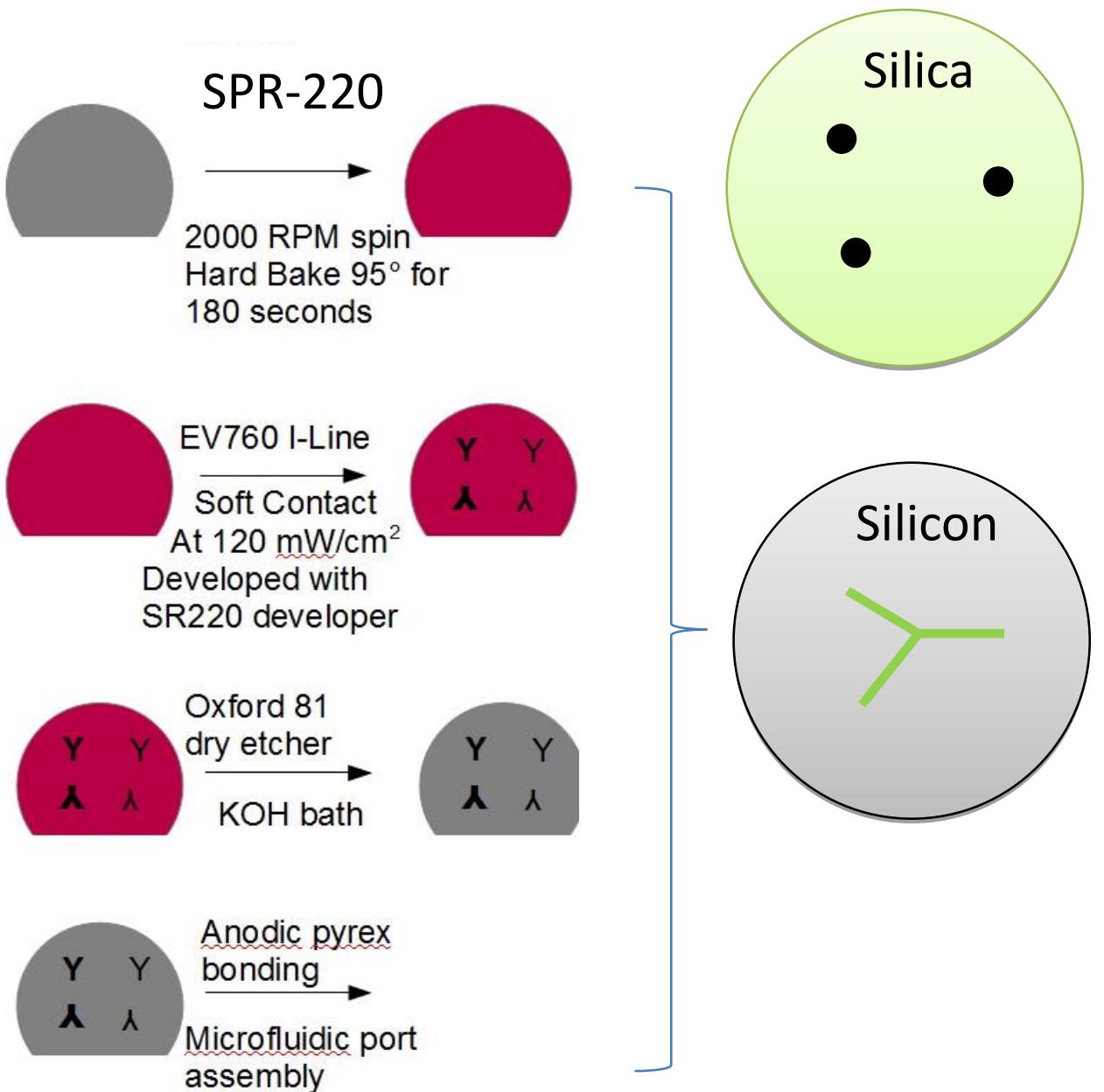

Figure 3.25: Fabrication of the microfluidic device. 
According to the design, the actual device was fabricated. The cover slide is a fluoroborate glass and the channel side is a silicon substrate. The key steps of the fabrication of the cover and channel slides are shown in figure 3.25. The device with different channel width has been made but not yet assembled and tested.

This device has provided a good platform to tune the assembly of nanoparticles and $\mathrm{KCl}$ matrix and to study the kinetics of the reducing process. However, due to the time line and schedule, this part of work may request the effort of future graduate students. Here the discussion of the benefit of the microfluidic device is to stimulate the interest of further studies.

\subsection{Conclusion}

As a conclusion, in this chapter, two main issues regarding this $\mathrm{Np}-\mathrm{KCl}$ method have been discussed. Firstly, Using $\mathrm{Pt}_{3} \mathrm{Fe}$ and $\mathrm{PtFe}$ intermetallic nanoparticles as examples, a surfactant-free $\mathrm{Np}-\mathrm{KCl}$ matrix method has been introduced for the synthesis of nanoparticles with controlled size and structure. In this method, the $\mathrm{Np}-\mathrm{KCl}$ assembly is formed in a one-pot reduction in THF at room temperature. $\mathrm{KCl}$ is an insoluble byproduct of the reaction and serves as a matrix that traps the nanoparticles to avoid particle agglomeration and to control the coalescence of nanoparticles during thermal annealing up to $600{ }^{\circ} \mathrm{C}$. By varying the molar ratio of metal precursors and $\mathrm{KCl}$, as well as the time and temperature of annealing, the final particle sizes and crystalline

order can be independently controlled. Secondly, by tracking of growth and motion of individual particles during thermal annealing of the $\mathrm{Np}-\mathrm{KCl}$ assemblies, the in-situ 
STEM studies of the $\mathrm{Np}-\mathrm{KCl}$ method have demonstrated that coalescence is the dominant mechanism of nanoparticle growth in the $\mathrm{KCl}$ matrix. This study also confirms that encasing nanoparticles in a $\mathrm{KCl}$ matrix significantly slows agglomeration as well as particle size growth.

In this Chapter, especially the TEM, STEM, and in-situ STEM work has been collaboratively done with Yingchao Yu and Houlin Xin in the Abruña group. Former group members Brian Leonard and Deli Wang, as well as the current DiSalvo group members of Minh Nguyen and Doug DeSario along with a number of undergraduates, who worked with myself and the people above, have all made important contributions to the development and understanding of the $\mathrm{Np}-\mathrm{KCl}$ method. I would not have succeeded without their cheerful, constant collaboration and their eagerness to discuss ideas. I would like to especially thank those undergraduates who worked directly with me: Kathryn Newton and Daniel Marshall.

\section{REFERENCES}

(1) DiSalvo, F. J.; Casado-Rivera, E.; Volpe, D. J.; Alden, L.; Lind, C.; Downie, C.; Vazquez-Alvarez, T.; Angelo, A. C. D.; Abruna, H. D. J Am Chem Soc 2004, 126, 4043.

(2) Abe, H.; Matsumoto, F.; Alden, L. R.; Warren, S. C.; Abruna, H. D.; DiSalvo, F. J. J Am Chem Soc 2008, 130, 5452.

(3) DiSalvo, F. J.; Roychowdhury, C.; Matsumoto, F.; Mutolo, P. F.; 
Abruna, H. D. Chem Mater 2005, 17, 5871.

(4) DiSalvo, F. J.; Ghosh, T.; Leonard, B. M.; Zhou, Q. Chem Mater 2010, 22, 2190.

(5) Wang, D. L.; Xin, H. L. L.; Hovden, R.; Wang, H. S.; Yu, Y. C.; Muller, D. A.; DiSalvo, F. J.; Abruna, H. D. Nat Mater 2013, 12, 81.

(6) Wang, D. L.; Yu, Y. C.; Xin, H. L. L.; Hovden, R.; Ercius, P.; Mundy, J. A.; Chen, H.; Richard, J. H.; Muller, D. A.; DiSalvo, F. J.; Abruna, H. D. Nano Lett 2012, 12, 5230 .

(7) Chen, H.; Wang, D. L.; Yu, Y. C.; Newton, K. A.; Muller, D. A.; Abruna, H.; DiSalvo, F. J. J Am Chem Soc 2012, 134, 18453.

(8) Kim, J.; Lee, Y.; Sun, S. H. J Am Chem Soc 2010, 132, 4996.

(9) Wikander, K.; Ekstroem, H.; Palmqvist, A. E. C.; Lindbergh, G. Electrochim Acta 2007, 52, 6848.

(10) Holby, E. F.; Sheng, W. C.; Shao-Horn, Y.; Morgan, D. Energ Environ Sci 2009, 2, 865 .

(11) Kim, J. M.; Rong, C. B.; Liu, J. P.; Sun, S. H. Adv Mater 2009, 21, 906.

(12) Yamamoto, S.; Morimoto, Y.; Tamada, Y.; Takahashi, Y. K.; Hono, K.; Ono, T.; Takano, M. Chem Mater 2006, 18, 5385.

(13) Yamamoto, S.; Morimoto, Y.; Ono, T.; Takano, M. Appl Phys Lett $2005,87$.

(14) Tamada, Y.; Yamamoto, S.; Takano, M.; Nasu, S.; Ono, T. Appl Phys Lett 2007, 90.

(15) Wang, D. L.; Subban, C. V.; Wang, H. S.; Rus, E.; DiSalvo, F. J.; 
Abruna, H. D. J Am Chem Soc 2010, 132, 10218.

(16) Bock, C.; Paquet, C.; Couillard, M.; Botton, G. A.; MacDougall, B. R. J Am Chem Soc 2004, 126, 8028.

(17) Wang, Y.; Ren, J. W.; Deng, K.; Gui, L. L.; Tang, Y. Q. Chem Mater $2000,12,1622$.

(18) Bonet, F.; Guery, C.; Guyomard, D.; Urbina, R. H.; Tekaia-Elhsissen, K.; Tarascon, J. M. Int J Inorg Mater 1999, 1, 47.

(19) Bonet, F.; Delmas, V.; Grugeon, S.; Urbina, R. H.; Silvert, P. Y.; Tekaia-Elhsissen, K. Nanostruct Mater 1999, 11, 1277.

(20) Strong, J.; Tuttle, T. R. J Phys Chem-Us 1973, 77, 533.

(21) Garrett, D. E. Handbook of lithium and natural calcium chloride : their deposits, processing, uses and properties; 1st ed.; Elsevier Academic Press: Amsterdam ; Boston, 2004.

(22) Alden, L. R.; Han, D. K.; Matsumoto, F.; Abruna, H. D.; DiSalvo, F. J. Chem Mater 2006, 18, 5591.

(23) Bonnemann, H.; Richards, R. M. Eur J Inorg Chem 2001, 2455.

(24) Basin, A. S.; Kaplun, A. B.; Meshalkin, A. B.; Uvarov, N. F. Russ J Inorg Chem+ 2008, 53, 1509.

(25) Warren, B. 1990.

(26) Kirkland, E. J.; Loane, R. F.; Silcox, J. Ultramicroscopy 1987, 23, 77.

(27) Crewe, A. V.; Wall, J.; Langmore, J. Science 1970, 168, 1338.

(28) Yu, T.; Xin, H.; Hovden, R.; Wang, D.; Rus, E.; Mundy, J. A.; Muller, D. A.; Abruña, H. A. Nano Lett 2011, ASAP.

(29) Muller, D. A.; Kourkoutis, L. F.; Murfitt, M.; Song, J. H.; Hwang, H. 
Y.; Silcox, J.; Dellby, N.; Krivanek, O. L. Science 2008, 319, 1073.

(30) Delalande, M.; Guinel, M. J. F.; Allard, L. F.; Delattre, A.; Le Bris, R.; Samson, Y.; Bayle-Guillemaud, P.; Reiss, P. J Phys Chem C 2012, 116, 6866.

(31) Yu, Y.; Xin, H. L.; Hovden, R.; Wang, D.; Rus, E.; Mundy, J. A.; Muller, D. A.; Abruña, H. D. Nano Lett 2011, Article ASAP. DOI:10.1021/nl203920s. (32) Xin, H. L.; Mundy, J. A.; Liu, Z.; Cabezas, R.; Hovden, R.; Kourkoutis, L. F.; Zhang, J.; Subramanian, N. P.; Makharia, R.; Wagner, F. T.; Muller, D. A. Nano Lett 2011, 12, 490.

(33) Yin, Y. D.; Rioux, R. M.; Erdonmez, C. K.; Hughes, S.; Somorjai, G. A.; Alivisatos, A. P. Science 2004, 304, 711.

(34) Chang, S. L. Y.; Barnard, A. S.; Dwyer, C.; Hansen, T. W.; Wagner, J. B.; Dunin-Borkowski, R. E.; Weyland, M.; Konishi, H.; Xu, H. The Journal of Physical Chemistry Letters 2012.

(35) Delalande, M. 1.; Guinel, M. J. F.; Allard, L. F.; Delattre, A.; Le Bris, R. m.; Samson, Y.; Bayle-Guillemaud, P.; Reiss, P. The Journal of Physical Chemistry $C 2012$.

(36) Bowker, M. Nat Mater 2002, 1, 205.

(37) Campbell, C. T.; Parker, S. C.; Starr, D. E. Science 2002, $298,811$.

(38) Karnesky, R. A.; Isheim, D.; Seidman, D. N. Appl Phys Lett 2007, 91.

(39) Sudbrack, C. K.; Ziebell, T. D.; Noebe, R. D.; Seidman, D. N. Acta Mater 2008, 56, 448.

(40) Granqvist, C. G.; Buhrman, R. A. J Catal 1976, 42, 477.

(41) Lifshitz, I. M.; Slyozov, V. V. J Phys Chem Solids 1961, 19, 35.

(42) Boyer, P.; Meunier, M. J Phys Chem C 2012, 116, 8014. 
(43) Lee, D.; Choi, M. J Aerosol Sci 2002, 33, 1.

(44) Xin, H. L.; Ercius, P.; Hughes, K. J.; Engstrom, J. R.; Muller, D. A. Appl Phys Lett 2010, 96.

(45) Zettergren, H.; Johansson, H. A. B.; Schmidt, H. T.; Jensen, J.; Hvelplund, P.; Tomita, S.; Wang, Y.; Martin, F.; Alcami, M.; Manil, B.; Maunoury, L.; Huber, B. A.; Cederquist, H. J. Chem. Phys. 2010, 133.

(46) Boyer, P.; Meunier, M. The Journal of Physical Chemistry C 2012, $116,8014$.

(47) Knight, J. B.; Vishwanath, A.; Brody, J. P.; Austin, R. H. Phys Rev Lett 1998, $80,3863$. 


\section{CHAPTER 4}

\section{THE NANOPARTICLE-KCl (Np-KCl) METHOD \\ PART II: RELEASING THE NANOPARTICLES FROM THE MATRIX}

\subsection{Introduction}

We have demonstrated that using the $\mathrm{Np}-\mathrm{KCl}$ method, structurally ordered intermetallic phases can be synthesized. This method can also provide the desired particle sizes with "clean" surfaces for catalytic reactions. However, in order to employ those intermetallic nanoparticles for catalytic applications, a transfer method is needed to release the nanoparticles from the $\mathrm{KCl}$ matrix and assemble them with the catalysts support materials, while preventing particle-particle agglomeration.

In this chapter, a detailed discussion on the transfer method will be presented. Since the common catalyst support for PEMFCs, Vulcan carbon, has different functional groups on the surface and we assume those will provide strong interactions with Pt-M nanoparticles, if the particle surface is clean. Therefore, Vulcan carbon is used as the support material without further modification. In order to release the nanoparticles from the $\mathrm{KCl}$ and uniformly bind them to the support materials, I explored two different strategies. In the first approach, I tried to develop a slow dissolution reaction of the $\mathrm{KCl}$ so that the nanoparticles could be slowly released from the matrix and then hopefully could be captured by the support materials before they aggregate. For little aggregation to occur, the particles released into solution must be at very low density, 
so that particle-particle collisons are much less frequent than particle-support collisions. In the second approach, I searched for a surfactant that would dissolve $\mathrm{KCl}$ and would be weakly bound to the nanoparticle surface, so that in high concentrations it prevents particle-particle agglomeration and so that it can be easily removed from the surface to bind to the support and then removed from the catalyst under mild conditions without leaving residues. . After the transfer step, the small molecule has to be completely removed to achieve a clean surface.

\subsection{The $\mathrm{BF}_{3}$ method}

The idea behind this $\mathrm{BF}_{3}$ method is that by exploiting a slow reaction with the $\mathrm{KCl}$, the nanoparticles can then be slowly released from the matrix. As a result of the slow releasing process, we hoped that the nanoparticles could be captured by the support materials before they aggregate.

$\mathrm{KCl}$ has an extremely low solubility in $\mathrm{THF}$. Therefore, as one can imagine, the equilibrium of equation 4.1 is very much on the left side meaning the free $\mathrm{Cl}^{-}$is very limited in the THF solution. Since $\mathrm{BF}_{3}$ is a very strong and hard Lewis acid, it provides interaction with $\mathrm{Cl}^{-}$and move the equilibrium of the reaction more toward the right side.

$$
\mathrm{KCl} \leftrightarrow \mathrm{K}^{+}+\mathrm{Cl}^{-} \quad \text { (equation } 4.1 \text { ) }
$$


A simple experiment was carried out to demonstrate this idea. $200 \mathrm{mg}$ of $\mathrm{KCl}$ was stirred overnight in a THF solution. As expected the $\mathrm{KCl}$ solid was not dissolved into THF solution. 15-crown-5, a good scavenger of $\mathrm{K}^{+}$in organic solvents, was then added into the solution and again stirred overnight, but still the $\mathrm{KCl}$ was not dissolved. However, on adding $0.1 \mathrm{ml}$ of $0.1 \mathrm{M} \mathrm{BF}_{3}$-THF into the resultant solution, the $\mathrm{KCl}$ dissolved in 20 minutes. Without adding $\mathrm{BF}_{3}$, there is very limited $\mathrm{Cl}^{-}$in the THF solution. On addition of only 15 -crown-5 the equilibrium between $\mathrm{K}^{+}$in solution and the crown apparently does not consume a significant amount of $\mathrm{K}^{+}$. Therefore the $\mathrm{KCl}$ was not dissolved overnight. However, the $\mathrm{BF}_{3}$ reacted with the $\mathrm{Cl}^{-}$in solution driving the dissolution of $\mathrm{KCl}$.

Besides providing interaction with free $\mathrm{Cl}^{-}$, the $\mathrm{BF}_{3}$ also serves as a halogen exchanger. Halogens on boron are known to exchange with free halogen anions in the solvent ${ }^{1}$. The reaction of $\mathrm{KCl}$ with excess $\mathrm{BF}_{3}$ in a $\mathrm{THF}$ solution in fact produces solid $\mathrm{KBF}_{4}$, but in the process releases the particles from the $\mathrm{KCl}$ matrix Shown in figure 4.1 are the $\mathrm{XRD}$ patterns of the products of the $\mathrm{KCl}$ and $\mathrm{BF}_{3}$ reaction in THF. The resultant solid was rinsed with THF before XRD. Clearly, $\mathrm{KBF}_{4}$ is generated.

Besides the strong Lewis acidity, another reason to use $\mathrm{BF}_{3}$ is that the $\mathrm{BF}_{3}$ precursor is a $\mathrm{BF}_{3}-\mathrm{THF}$ adduct, which is very stable both in air and under $\mathrm{Ar}$ in the glovebox. After learning of the reaction between $\mathrm{BF}_{3}$ and $\mathrm{KCl}$, a series of experiments were carried out to transfer the nanoparticles from the $\mathrm{KCl}$ matrix to carbon support in THF solutions that contain of $\mathrm{BF}_{3}$. The $\mathrm{Np}-\mathrm{KCl}$ used here is the PtFe-7KCl-600 ${ }^{\circ} \mathrm{C}$ 12hours. 


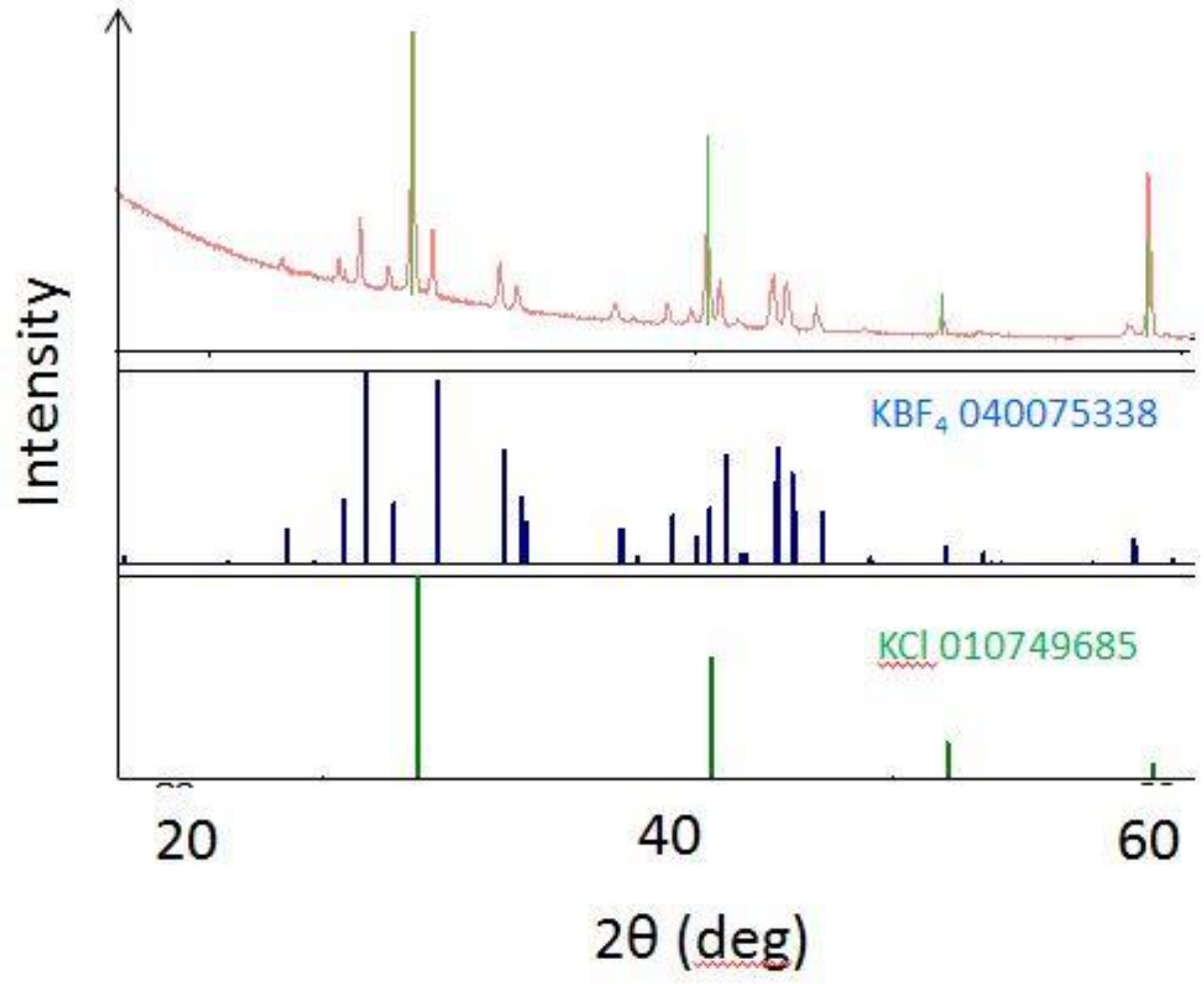

Figure 4.1: The $\mathrm{XRD}$ pattern of the product of the reaction of $\mathrm{KCl}$ and $\mathrm{BF}_{3}$ in a $\mathrm{THF}$ solution. The peak positions for $\mathrm{KBF}_{4}$ are shown at the bottom as blue tick marks with PDF card No. 040075338. The peak positions for $\mathrm{KCl}$ are shown at the bottom as green tick marks with PDF card No. 010749685 


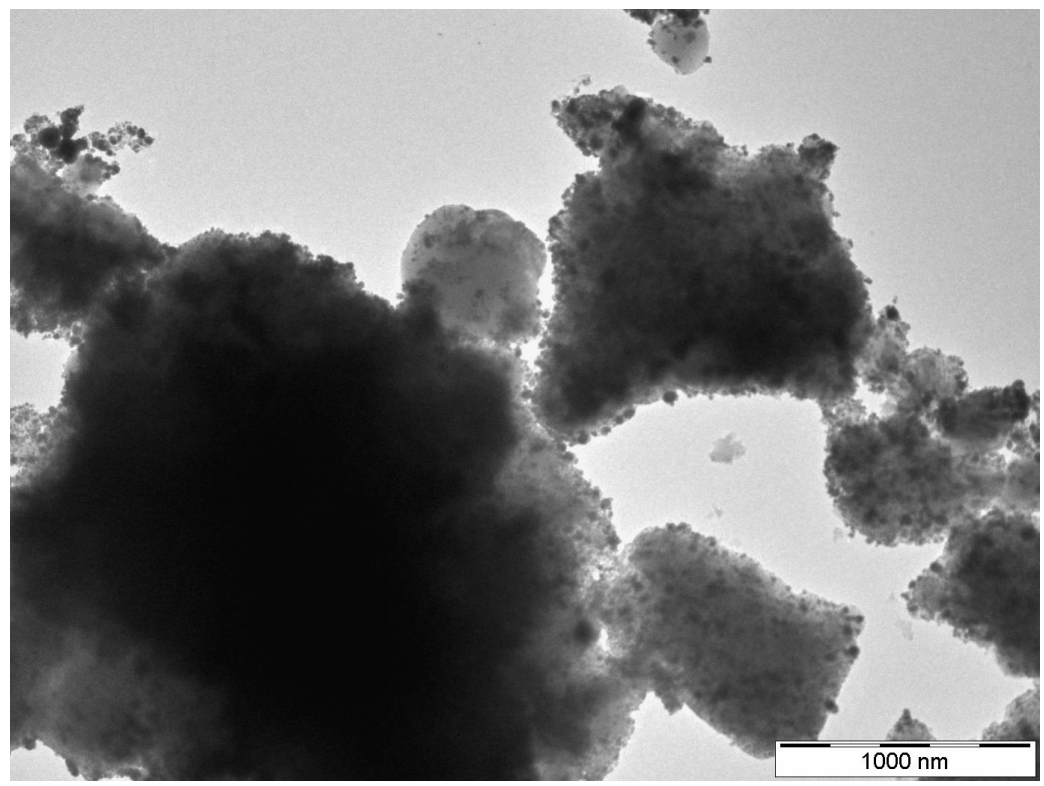

Figure 4.2: The PtFe-7KCl-600 ${ }^{\circ} \mathrm{C}-12$ hours sonicated for 4 hrs in THF solution without adding $\mathrm{BF}_{3}$.

The reaction was conducted under sonication. Firstly, to confirm the stability of the $\mathrm{Np}-\mathrm{KCl}$ assembly, they support and the $\mathrm{Np}-\mathrm{KCl}$ matric were sonicated for 4 hours in a THF solution. Shown in figure 4.2 is a TEM image of the product after sonication. As indicated by the TEM image, The $\mathrm{Np}-\mathrm{KCl}$ assembly is indeed stable under the reaction condition. The nanoparticles did not release from the $\mathrm{KCl}$ matrix. 


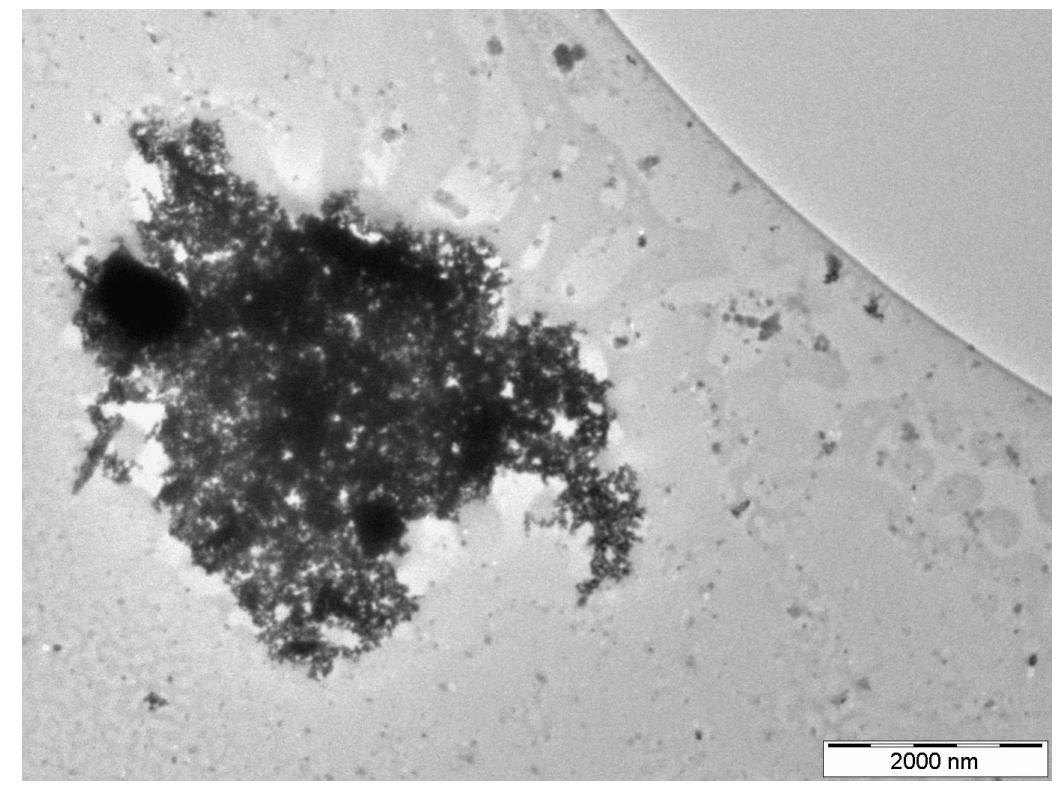

Figure 4.3: The PtFe-7KCl-600 ${ }^{\circ} \mathrm{C}-12$ hours sonicated for 4 hrs in THF solution after adding $\mathrm{BF}_{3}$.

$\mathrm{Next}, \mathrm{BF}_{3}$ was introduced to the $\mathrm{Np}-\mathrm{KCl}$ solution and sonicated for 4 hours. As shown in figure 4.3, in the TEM image, the nanoparticles heavily aggregated. Since there is nothing else in the solution to stabilize the released nanoparticles, they agglomerated. Therefore, a combination of oleic acid and hexadecanethiol (1:1) was introduced as surfactant to stabilize the released particles. As a control experiment, these surfactants were sonicated with $\mathrm{Np}-\mathrm{KCl}$ in a $\mathrm{THF}$ solution without adding $\mathrm{BF}_{3}$ As shown in figure 4.4, the TEM image of the product showed no releasing of the particles. 


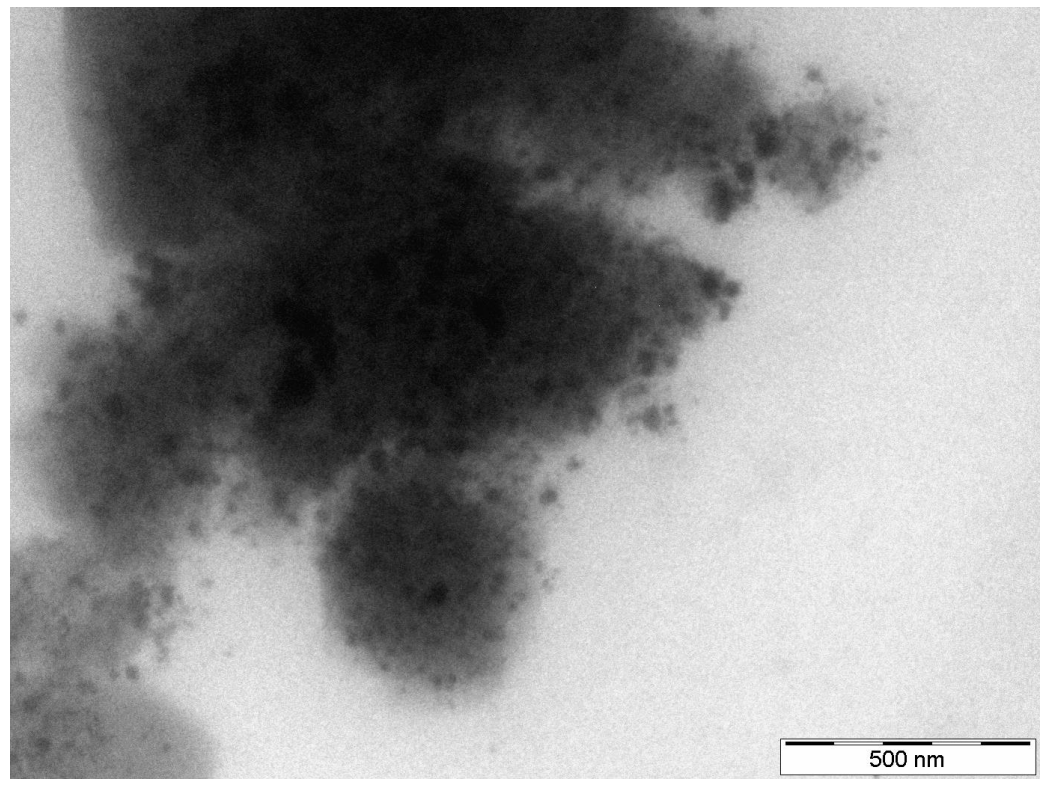

Figure 4.4: The PtFe-7KCl-600 ${ }^{\circ} \mathrm{C}-12$ hours sonicated for $4 \mathrm{hrs}$ in THF solution with oleic acid : hexadecanethiol 1:1 and without adding $\mathrm{BF}_{3}$. 

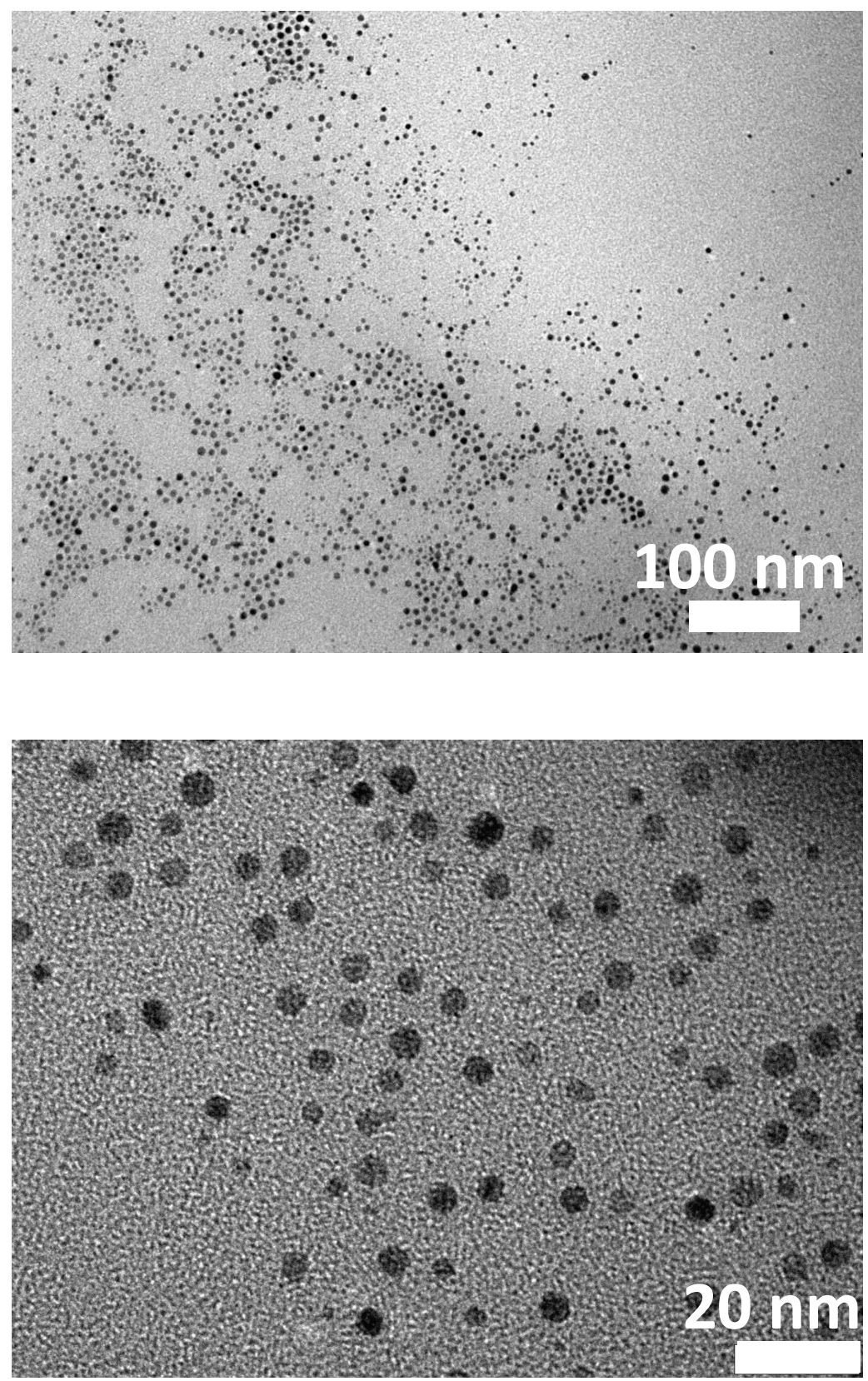

Figure 4.5: The PtFe-7KCl-600 ${ }^{\circ} \mathrm{C}-12$ hours sonicated for 4 hrs in THF solution with adding both oleic acid : hexadecanethiol 1:1 and $\mathrm{BF}_{3}$. 
By adding both the surfactants and the BF3-THF adduct, the PtFe nanoparticles are clearly released from the $\mathrm{KCl}$ matrix and captured by the surfactants (figure 4.5). The results of these experiments suggest that the BF3 can be used to release the nanoparticles from the $\mathrm{KCl}$ matrix. The next step was to replace the surfactants by Vulcan carbon support. Since the amount of nanoparticles can be calculated from the PtFe-7KCl stoichiometry, the mass of carbon support to achieve a $20 \mathrm{wt} \%$ of loading of the nanoparticles is easily determined and used. TEM images of the resultant powders are shown in figure 4.6. Clearly the loading is way less than $20 \mathrm{wt} \%$.

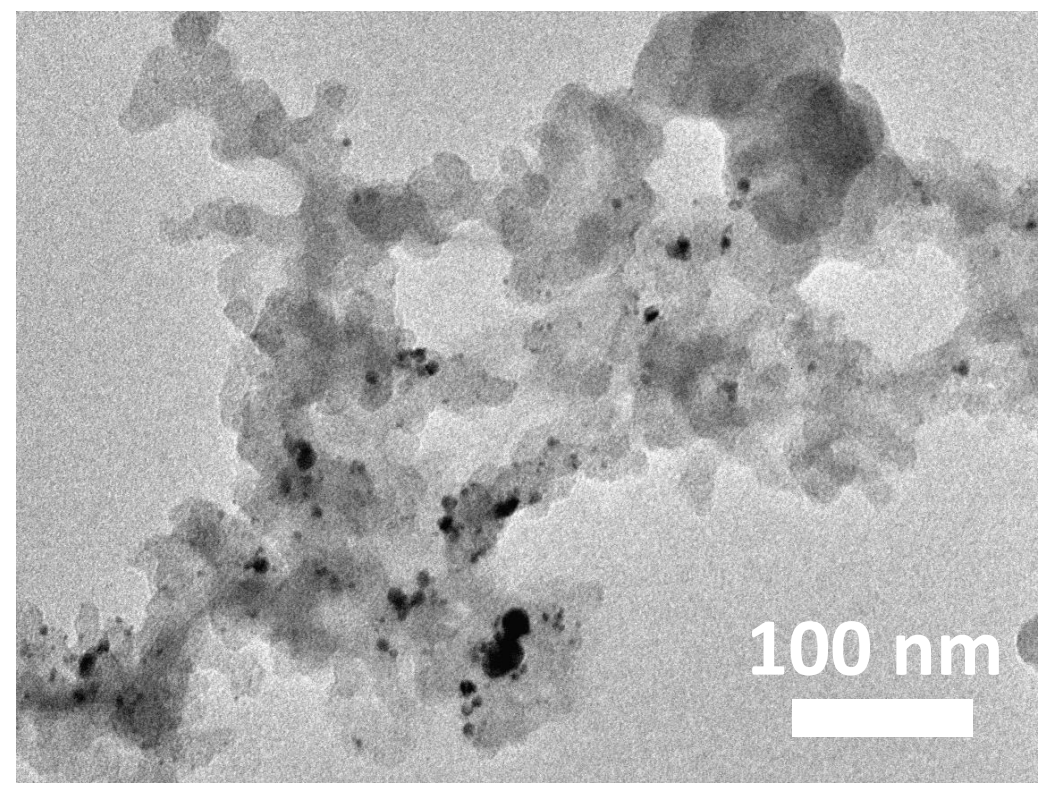

Figure 4.6: The PtFe-7KCl- $600{ }^{\circ} \mathrm{C}-12$ hours sonicated for $4 \mathrm{hrs}$ in THF solution with adding both $\mathrm{BF}_{3}$ and Vulcan carbon targeting at a $20 \mathrm{wt} \%$ loading after transfer. 
The reason for this failure is not fully understood. So this led us to the second method which had much better outcomes.

\subsection{The Ethylene Glycol method}

The idea behind this ethylene glycol method is to use a small weak surfactant molecule, which can temporarily stabilize the nanoparticles, to release the nanoparticles from the matrix and prevent their aggregation before bonding to the carbon support. After the transfer step, the small molecule needs to be able to be completely removed to achieve a clean surface.

Since $\mathrm{KCl}$ has a solubility of $37 \mathrm{~g}$ in $\mathrm{H}_{2} \mathrm{O}$, the transfer process was first conducted using $\mathrm{H}_{2} \mathrm{O}$ as the solvent. To achieve a more uniform distribution of the nanoparticles after transferring to the Vulcan carbon support, a solid sonicator (Microson ultrasonic cell disruptor XL) was employed. In a typical transfer experiment, the Vulcan carbon was first sonicated with $\mathrm{H}_{2} \mathrm{O}$ for 5 minutes to make a uniform suspension and then added to the scintillation vial holding the $\mathrm{Np}-\mathrm{KCl}$ powder. The mixture was then sonicated for another 8 minutes, after which the mixture was stirred overnight. The resultant mixture was then filtered out and washing extensively with $\mathrm{H}_{2} \mathrm{O}$ and dried out for different characterization measurement. The transfers were targeted at $10 \mathrm{wt} \%$ loading of nanoparticles on the carbon support. Therefore in a typical transfer, $5.15 \mathrm{mg}$ of Pt3Fe_80KCl_600 ${ }^{\circ} \mathrm{C} \_12$ hours and $4.5 \mathrm{mg}$ of carbon black were used. Since the tip 
of the solid sonicator used was small, 3 to $4 \mathrm{ml}$ of solvent was usually used. Under such conditions different solvents were used to examine the transfer process.

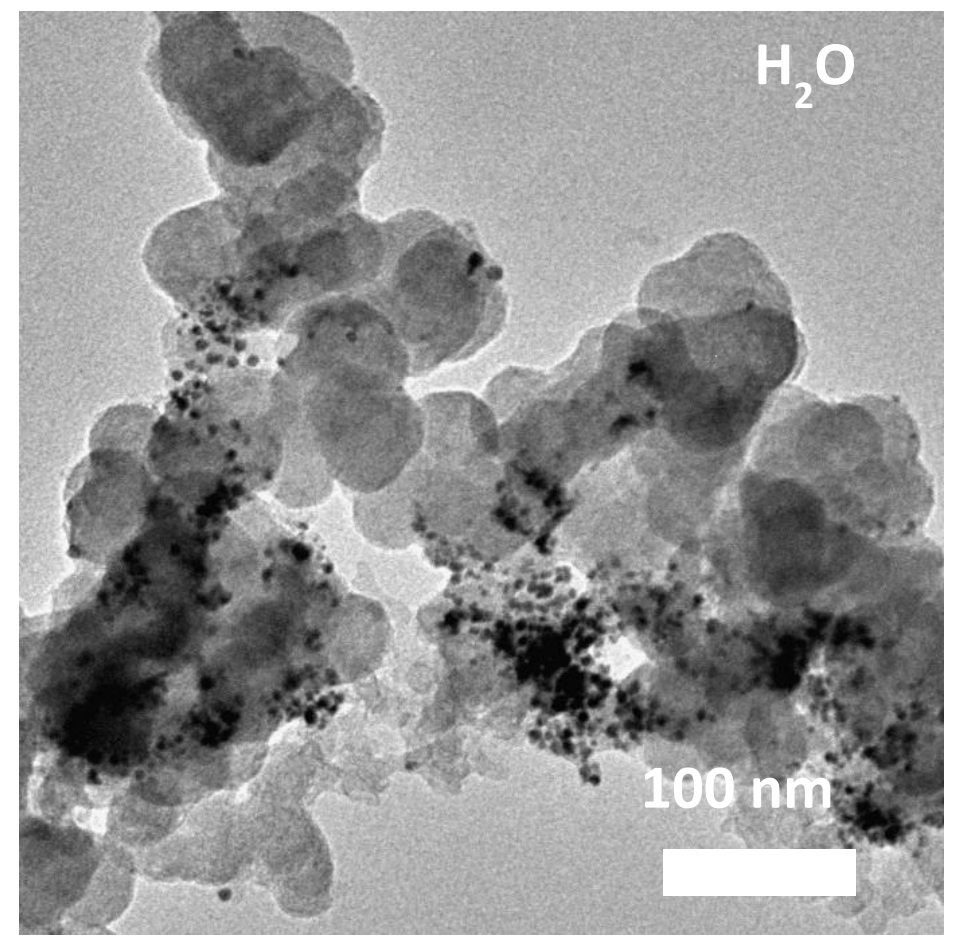

Figure 4.7: TEM image of 10 wt $\% \mathrm{Pt}_{3} \mathrm{Fe} / \mathrm{C}$ from transferring $\mathrm{Pt}_{3} \mathrm{Fe}-80 \mathrm{KCl}-600{ }^{\circ} \mathrm{C}$ 12hr to Vulcan X72 carbon black in $\mathrm{H}_{2} \mathrm{O}$.

Shown in figure 4.7 is the transferred $10 \mathrm{wt} \% \mathrm{Pt}_{3} \mathrm{Fe} / \mathrm{C}$ using $\mathrm{H}_{2} \mathrm{O}$ as the solvent. Clearly the nanoparticles are aggregated and the distribution is very bad. This result is not unexpected. The $\mathrm{KCl}$ has a really high solubility in water and water is not known 
to be a stabilizing ligand for Pt nanoparticles. Therefore the solvation process could be very fast under sonication and since there were no surfactants or small molecules to stabilize the nanoparticles, the small particles were then quickly released from the matrix and agglomerated together before they are be captured by the carbon support. As a result they were heavily aggregated.

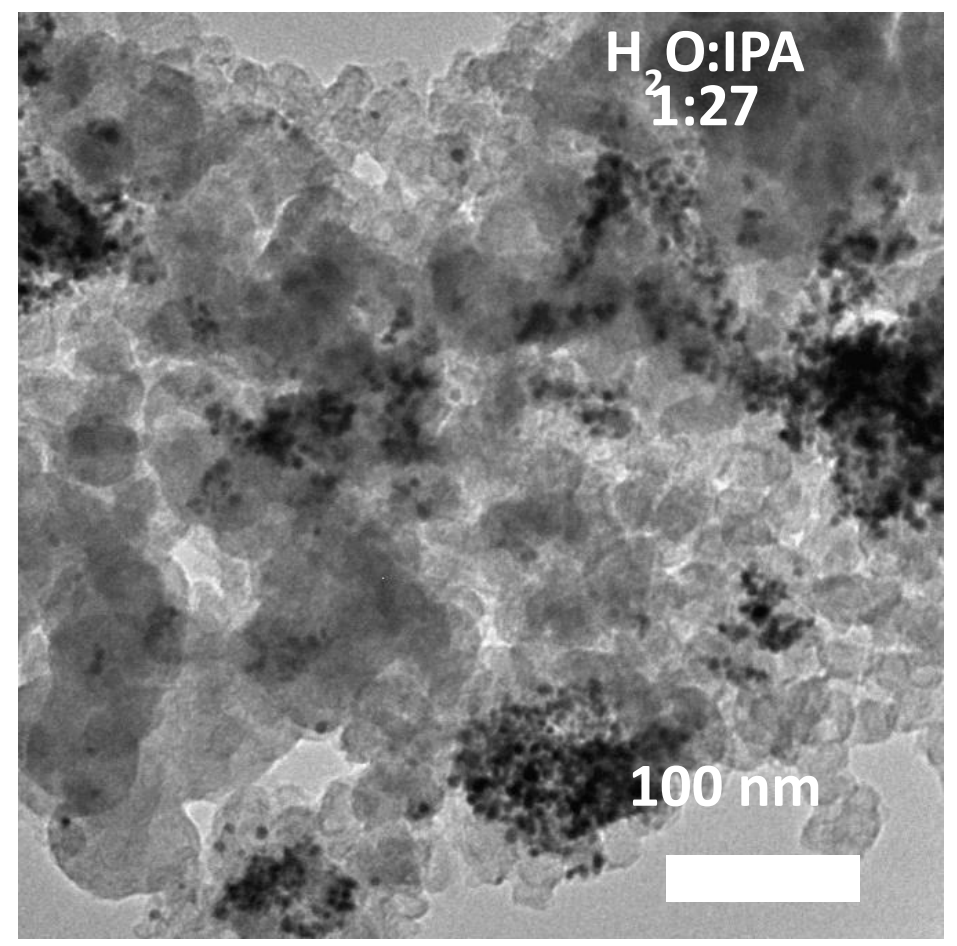

Figure 4.8: TEM image of 10 wt $\% \mathrm{Pt}_{3} \mathrm{Fe} / \mathrm{C}$ from transferring $\mathrm{Pt}_{3} \mathrm{Fe}-80 \mathrm{KCl}-600{ }^{\circ} \mathrm{C}$ $12 \mathrm{hr}$ to Vulcan X72 carbon black in a mixture of $\mathrm{H}_{2} \mathrm{O}$ and Isopropanol with a volume ratio of 1:27. 
Since $\mathrm{KCl}$ has a high solubility in water (about $25 \mathrm{~g} \mathrm{KCl}$ per $100 \mathrm{~g} \mathrm{H}_{2} \mathrm{O}$ ) but an extremely low solubility in most organic solvents, a mixture of $\mathrm{H}_{2} \mathrm{O}$ and isopropanol with different volume ratio was also explored as the transfer agent. However, as shown in figure 4.8 , the nanoparticles are still heavily aggregated after the transfer step. Therefore the nanoparticles have to be at least temporarily stabilized during this transfer process to prevent them from aggregation. Several other transfer agents were then tried, including ethylene glycol, which turned out to be a very good candidate. Ethylene glycol was known to be a weak surfactant, since it is the solvent of choice in the polyol process, which produces un-agglomerated Pt particles without the addition of other surfactants, but can then be washed away in water ${ }^{2,3}$ (see figure 4.9) As luck would have it, $\mathrm{KCl}$ also has moderate solubility in ethylene glycol ${ }^{4}$ (see figure 4.10).

When using ethylene glycol as the transfer agent, the nanoparticles were transferred to the carbon support without significant agglomeration and with good dispersion (figure 4.9). Similar results are obtained with mixed water ethylene glycol solutions containing up to 50 mole $\%$. This result confirms that the nanoparticles can stabilized during transfer. ${ }^{2,3}$. 

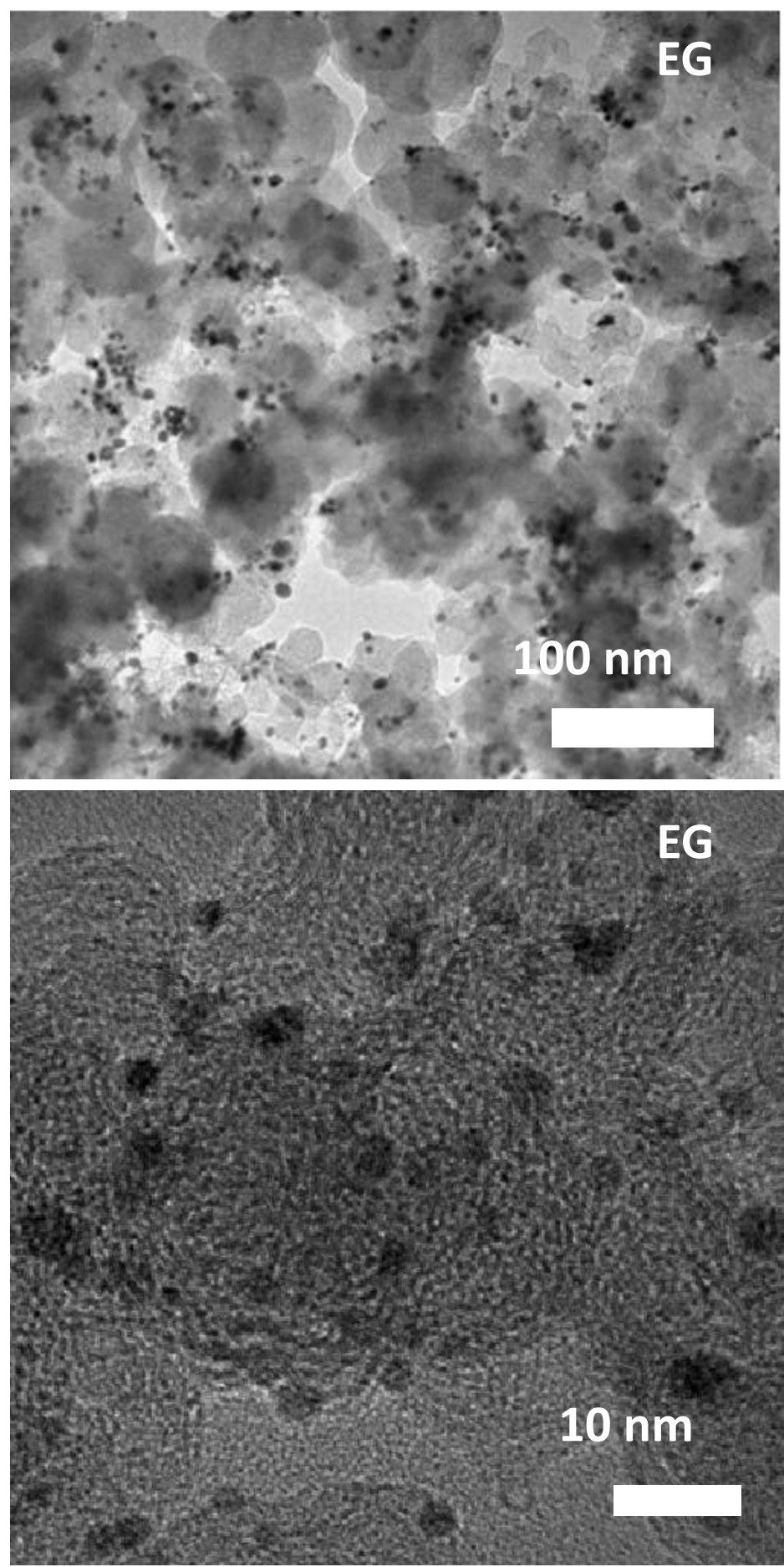

Figure 4.9: TEM image of 10 wt $\% \mathrm{Pt}_{3} \mathrm{Fe} / \mathrm{C}$ from transferring $\mathrm{Pt}_{3} \mathrm{Fe}-80 \mathrm{KCl}-600{ }^{\circ} \mathrm{C}$ $12 \mathrm{hr}$ to Vulcan X72 carbon black in ethylene glycol. 


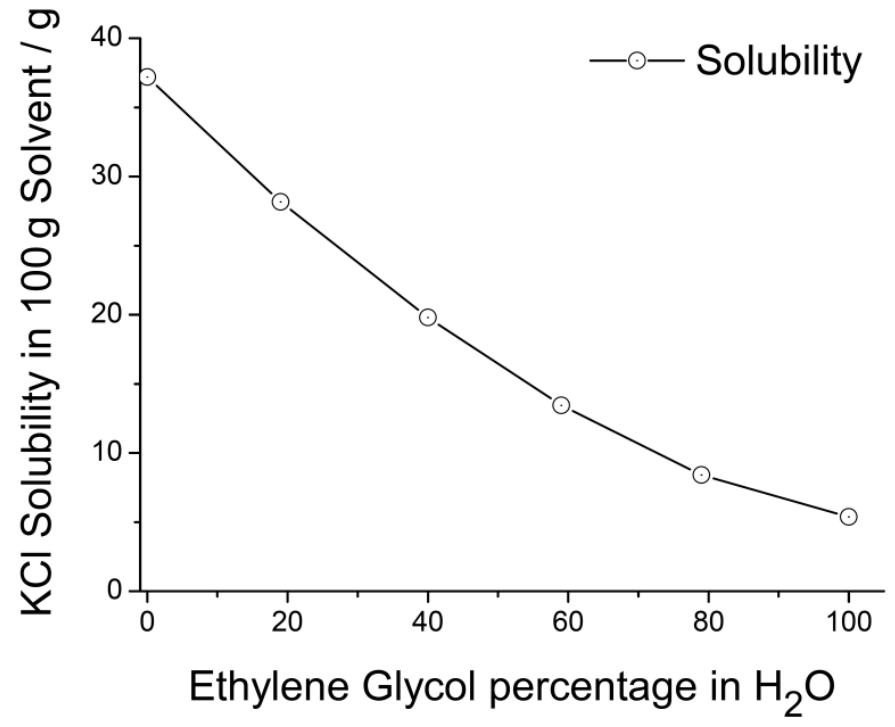

Figure 4.10: the solubility of $\mathrm{KCl}$ in the mixture of ethylene glycol and water ${ }^{4}$.

Since ethylene glycol is used as both the reducing agent and protecting ligand for nanoparticle synthesis via the polyol process, it has been well studied. ${ }^{2,3}$ These studies showed that ethylene glycol can be fully decomposed electrochemically at high potentials or by heat treatment below $175{ }^{\circ} \mathrm{C}$. Temperature programmed desorption (TPD) studies and density functional theory (DFT) calculations ${ }^{5-8}$ showed that on different Pt based mono- or bi-metallic surfaces, the ethylene glycol completely decomposes under vacuum, to $\mathrm{H}_{2}, \mathrm{CO}$ or $\mathrm{CO}_{2}$ by $175{ }^{\circ} \mathrm{C}$. At such temperatures, obvious nanoparticle sintering does not occur on carbon black ${ }^{9}$ and a relatively clean surface is obtained. 

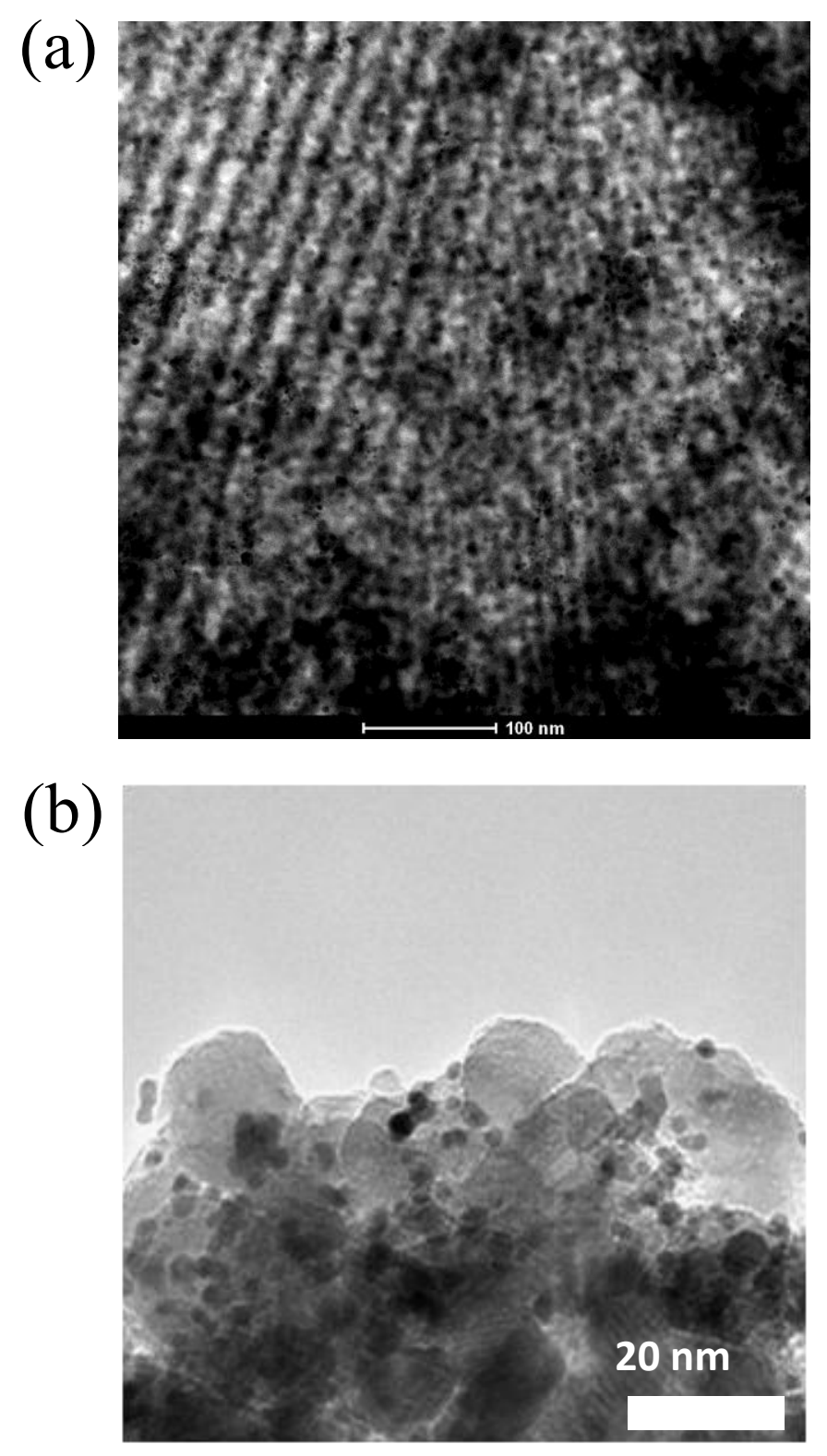

Figure 4.11: TEM images of $\mathrm{Pt}_{3} \mathrm{Fe}$ transferred to different support materials using ethylene glycol as the transfer solvent. (a) on $\mathrm{Ti}_{0.8} \mathrm{Nb}_{0.2} \mathrm{~N}$; (b) on $\mathrm{TiO}_{2}$.

Since the nanoparticles are stabilized by the ethylene glycol during the transfer step, a similar process can be used to transfer the nanoparticles to different support materials, 
assuming that the nanoparticles have sufficient binding energy to that material . Shown in figure $4.11 \mathrm{a}$ is the TEM image of the $\mathrm{Pt}_{3} \mathrm{Fe}$ that transferred from $\mathrm{Pt}_{3} \mathrm{Fe}$ $80 \mathrm{KCl}-600{ }^{\circ} \mathrm{C}-12 \mathrm{hr}$ to $\mathrm{Ti}_{0.8} \mathrm{Nb}_{0.2} \mathrm{~N}$ using ethylene glycol as transfer agent. Similarly, in figure $4.11 \mathrm{~b}$, the $\mathrm{Pt}_{3} \mathrm{Fe}$ nanoparticles were transferred to oxide materials using the same procedure. These results demonstrate that this ethylene glycol based transfer process is a general method

\subsection{Electrochemical measurements of supported catalysts}

10 wt $\% \mathrm{Pt}_{3} \mathrm{Fe}-80 \mathrm{KCl}-600{ }^{\circ} \mathrm{C}-12 \mathrm{hr}$ supported by Vulcan $\mathrm{X} 72$ carbon has been prepared and processed using the ethylene glycol based transfer method mentioned above. The TEM image of the product $\left(\mathrm{Pt}_{3} \mathrm{Fe} / \mathrm{C}\right)$ is shown in figure 4.9. As expected, little aggregation of the nanoparticles or changes in particle sizes was observed. To be sure that most of the nanoparticles are bound to the carbon, the loading of the $\mathrm{Pt}_{3} \mathrm{Fe}$ was independently determined by thermogravimetric analysis (TGA) of the product by heating in air to remove the carbon as $\mathrm{CO}_{2}$ and oxidizing the $\mathrm{Fe}$ to $\mathrm{Fe}_{2} \mathrm{O}_{3}$. The TGA data showed a Pt ${ }_{3} \mathrm{Fe}$ loading of $10 \mathrm{wt} \%$ (figure 4.12).

After confirming the loading, the ORR activity of the supported catalysts was tested. Electrochemical experiments were conducted in $0.1 \mathrm{M} \mathrm{HClO}_{4}$ at room temperature with a rotating disk electrode using a Solartron electrochemistry station. A reversible hydrogen electrode (RHE) and a Pt wire were used as reference and counter electrodes, respectively. Catalysts inks were prepared by mixing the catalysts with 


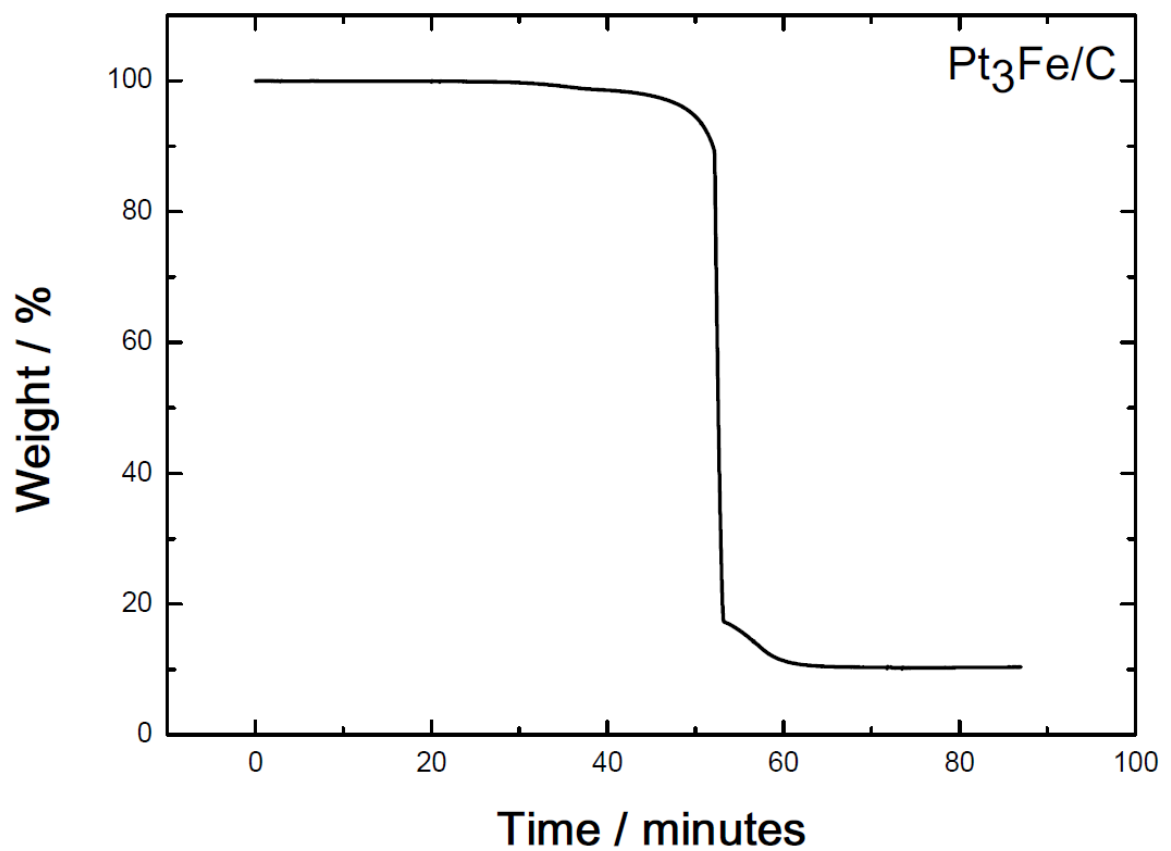

Figure 4.12: Thermogravimetric analysis (TGA) of $\mathrm{Pt}_{3} \mathrm{Fe}-80 \mathrm{KCl}-600{ }^{\circ} \mathrm{C}-12 \mathrm{hr}$ supported on carbon black $\left(\mathrm{Pt}_{3} \mathrm{Fe} / \mathrm{C}\right.$ at a target value of $10 \mathrm{wt} \%$ metals). The TGA was conducted in flowing air. The sample was heated up to $550{ }^{\circ} \mathrm{C}$ at $10{ }^{\circ} \mathrm{C} / \mathrm{min}$ and held at $550{ }^{\circ} \mathrm{C}$ for 30 minutes. The carbon black was oxidized to $\mathrm{CO}_{2}$. At the given loading the weight gain from oxidation of $\mathrm{Fe}$ is $0.37 \mathrm{wt} \%$ of the sample mass, if all the $\mathrm{Fe}$ is oxidized to $\mathrm{Fe}_{2} \mathrm{O}_{3}$. At this point we are not sure to what extent the $\mathrm{Fe}$ is oxidized. The TGA data suggests the loading of $\mathrm{Pt}_{3} \mathrm{Fe}$ on carbon black is close to 10 wt $\%$. 
Nafion (0.05 wt \% Nafion dissolved in ethanol). The ink solutions were deposited on glassy carbon electrodes and dried in air to serve as the working electrode. For the specific activity evaluation, the electrochemical surface areas (ECSA) of Pt were calculated by integrating the average areas of hydrogen adsorption and desorption regions, using a conversion factor of $200 \mu \mathrm{Ccm}^{-2}$. The ORR polarization curves were obtained by sweeping the potential from +0.1 to $+1.1 \mathrm{~V}$ anodically at a scan rate of 5 $\mathrm{mV} \mathrm{s}^{-1}$ and rotation rate of $1600 \mathrm{rpm}$. The specific activities for mass and surface area were established at $0.85 \mathrm{~V}$ and $0.90 \mathrm{~V}$ based on ORR polarization curves using the Koutecky-Levich equation, which can be described as:

$\frac{1}{I}=\frac{1}{I_{d}}+\frac{1}{I_{k}}$

Where $I$ is the overall current density, which is the experimentally measured current; $I_{\mathrm{d}}$ is the diffusion-limited current density, which we choose as the current at $0.4 \mathrm{~V}$ measured from the ORR polarization curves, and $I_{k}$ is the kinetic current density at the potential of interest. All electrochemical measurements were conducted at $21{ }^{\circ} \mathrm{C}$.

The cyclic voltammograms (CVs) of $\mathrm{Pt}_{3} \mathrm{Fe} / \mathrm{C}$ and $20 \mathrm{wt} \% \mathrm{Pt} / \mathrm{C}$ are shown in figure 4.13. Currents were normalized to the electrochemical surface areas (ECSA) of Pt which were calculated by measuring the average charge associated with the adsorption and desorption of hydrogen between +0.05 and $+0.40 \mathrm{~V}$ (figure 4.14) with a conversion factor of $200 \mu \mathrm{Ccm}^{-2}$ for the adsorption of a hydrogen monolayer. ${ }^{10,11}$ 


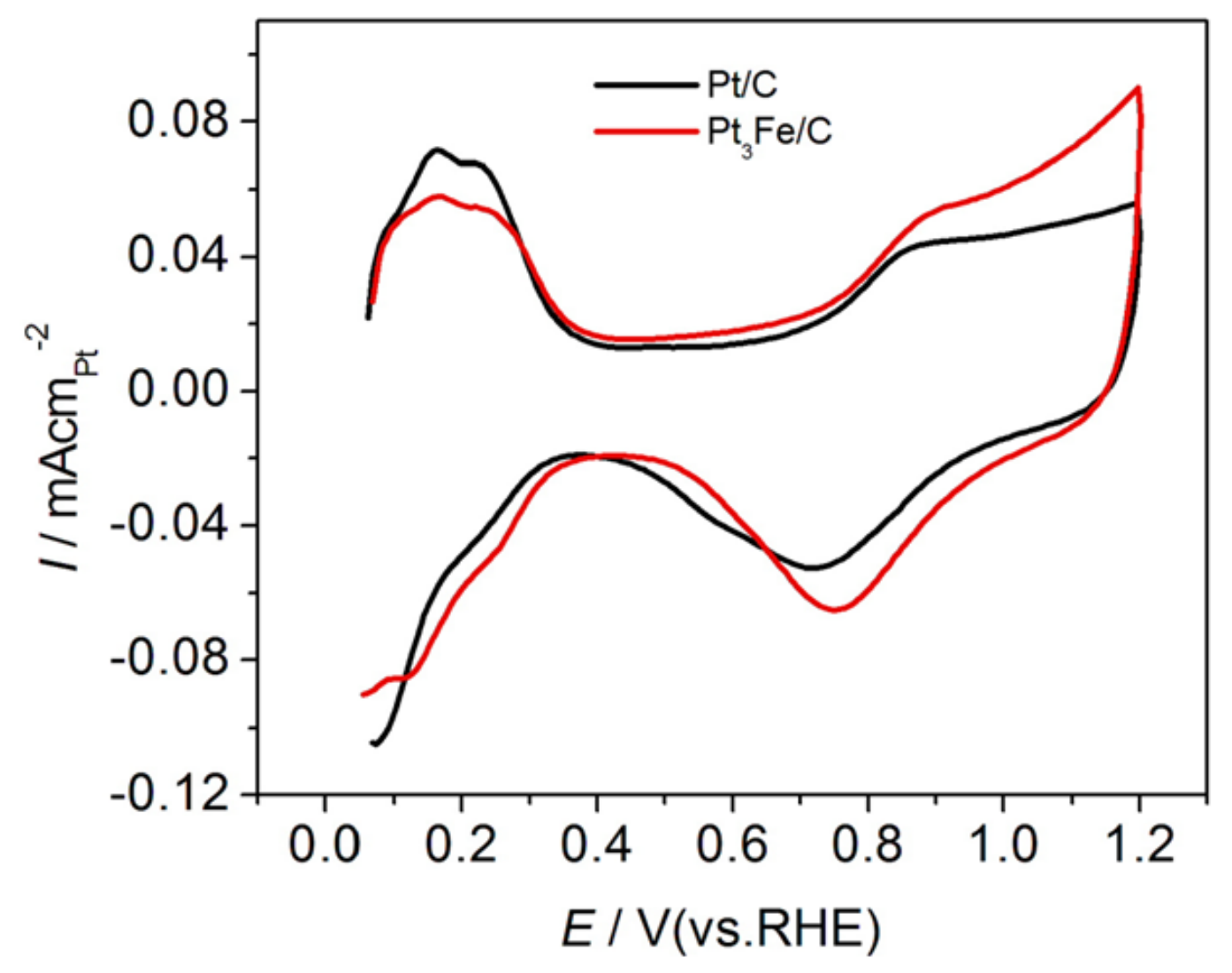

Figure 4.13: The Cyclic Voltammetric profiles of $\mathrm{Pt}_{3} \mathrm{Fe} / \mathrm{C}$ and $\mathrm{Pt} / \mathrm{C}$ on a glassy carbon electrode in $0.1 \mathrm{M} \mathrm{HClO} 4$ solution. Currents were normalized to the electrochemical surface areas of Pt. 


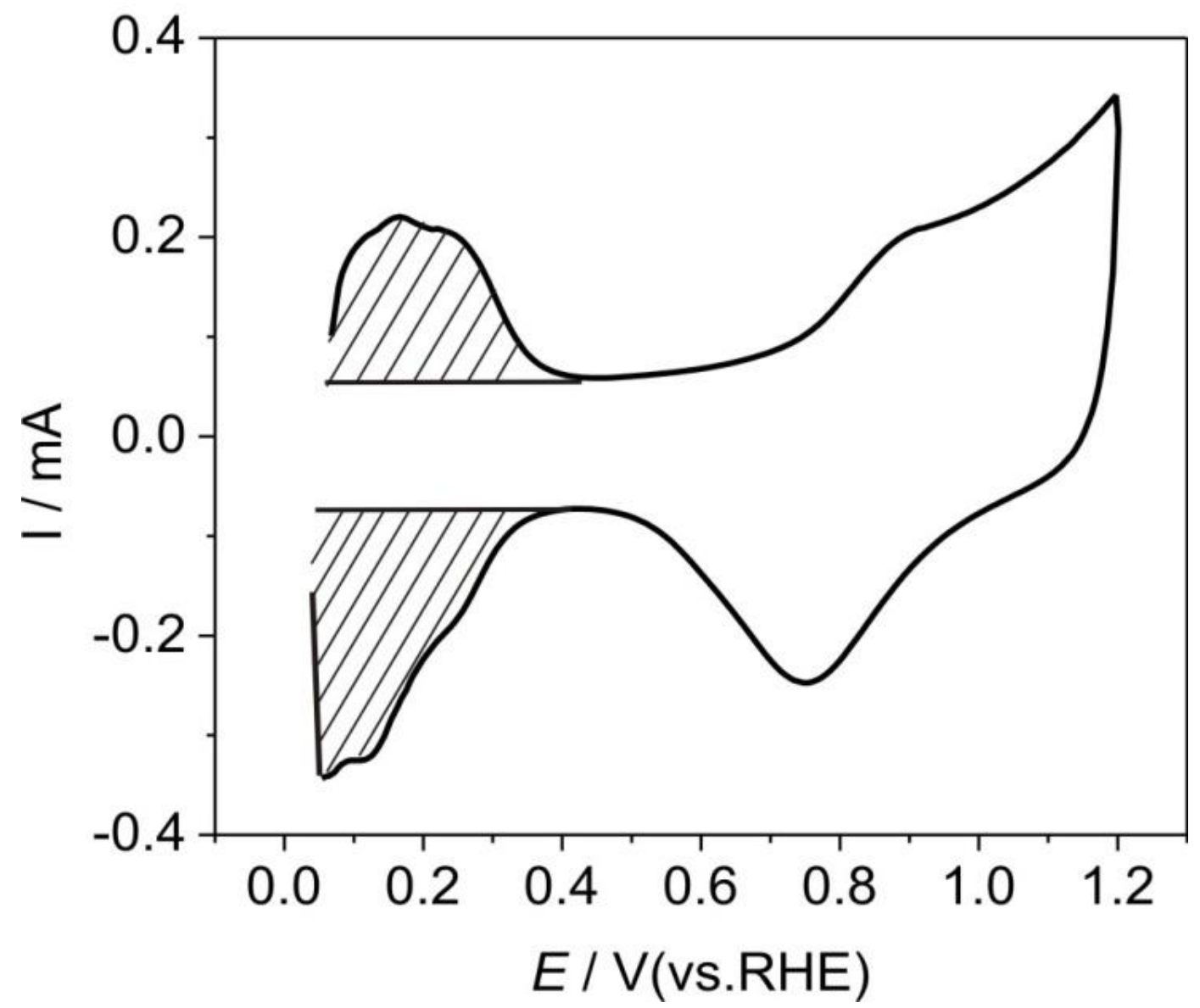

Figure 4.14: Representative $\mathrm{CV}$ curve of $\mathrm{Pt}_{3} \mathrm{Fe} / \mathrm{C}$ for electrochemical surface area (ECSA) calculation. 
We assumed that the Fe atoms in the top few monolayers of the $\mathrm{Pt}_{3} \mathrm{Fe}$ are leached out of the surface at the high potentials of oxygen reduction, so that core-shell materials are obtained with an outer skin of pure Pt, as previously reported. We further assumed that the hydrogen adsorption density on the core-shell particles is the same as on pure Pt. One prominent feature from the $\mathrm{CVs}$ is that in the cathodic scan, the peak potential for $\mathrm{Pt}_{3} \mathrm{Fe} / \mathrm{C}$ catalyst associated with $\mathrm{Pt}$ surface oxide reduction shifts to a more positive potential compared with pure $\mathrm{Pt} / \mathrm{C}$. It has been suggested that the peak potential is related to the binding strength of oxygen. A positive shift of the peak potential reflects a decrease in the adsorption strength for the oxygen species ${ }^{3}$.

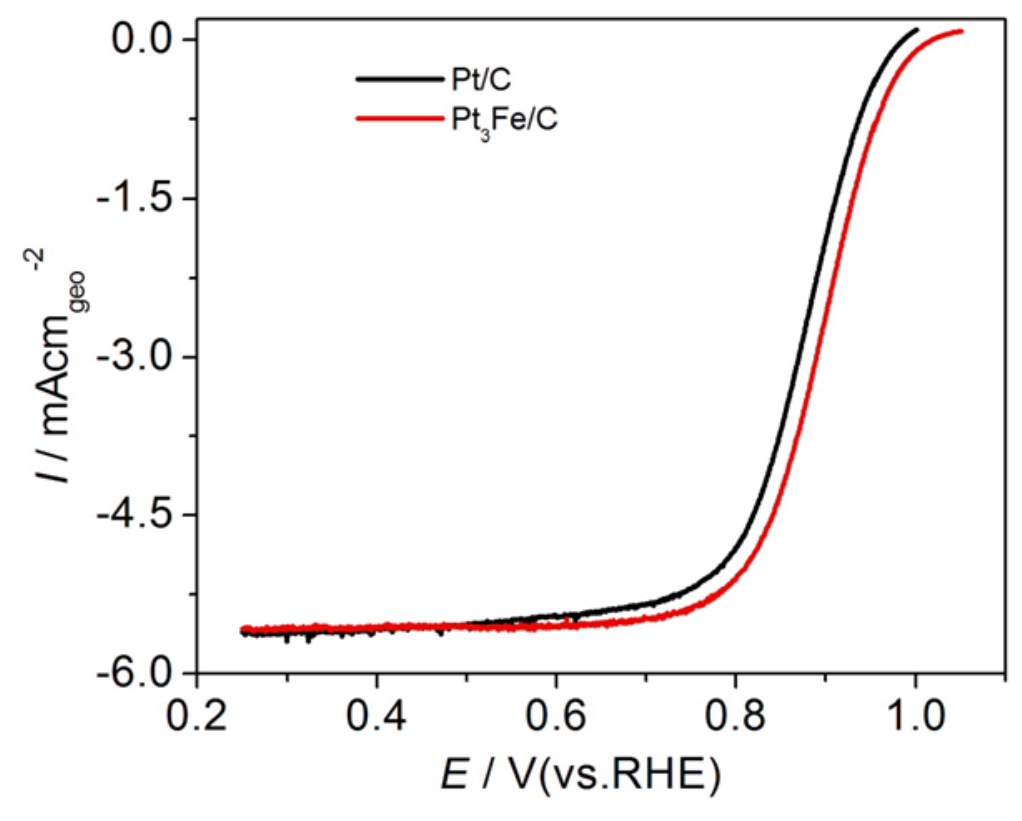

Figure 4.15: Polarization curves of the ORR on the two carbon supported catalysts.

Current densities were normalized to the geometric surface area of the electrode. 
Figure 4.15 shows linear sweep voltammograms (LSV) for $\mathrm{Pt} / \mathrm{C}$ and $\mathrm{Pt}_{3} \mathrm{Fe} / \mathrm{C}$ catalysts in $\mathrm{O}_{2}$-saturated $0.1 \mathrm{M} \mathrm{HClO}$, using a rotating disk electrode at $1600 \mathrm{rpm}$. Current densities were normalized to the geometric surface area of the electrodes. $\mathrm{The}^{\mathrm{Pt}} \mathrm{Fe}_{3} \mathrm{Fe}$ catalyst exhibited a slightly smaller overpotential than a Pt/C catalyst for ORR. Halfwave potentials for the $\mathrm{Pt} / \mathrm{C}$ and $\mathrm{Pt}_{3} \mathrm{Fe} / \mathrm{C}$ catalysts were $0.875 \mathrm{~V}$ and $0.900 \mathrm{~V}$, respectively. The $\mathrm{Pt}_{3} \mathrm{Fe} / \mathrm{C}$ catalyst showed a $25 \mathrm{mV}$ positive shift compared to that of $\mathrm{Pt} / \mathrm{C}$. In the mass transport limited region, the current density was almost the same for both the $\mathrm{Pt}_{3} \mathrm{Fe} / \mathrm{C}$ and $\mathrm{Pt} / \mathrm{C}$ electrode, indicating that the ORR on the $\mathrm{Pt}_{3} \mathrm{Fe} / \mathrm{C}$ electrode proceeds mainly through the direct 4-electron pathway as on a $\mathrm{Pt} / \mathrm{C}$ electrode. The kinetic current density $\left(\mathrm{j}_{\mathrm{k}}\right)$ is often used to compare the intrinsic activities of the catalysts. There are two ways to report the $\mathrm{j}_{\mathrm{k}}$ : specific activity and mass activity. Figure 4.16a shows the specific activity of $\mathrm{Pt} / \mathrm{C}$ and $\mathrm{Pt}_{3} \mathrm{Fe} / \mathrm{C}$ catalysts obtained from ORR polarization. Further experimental details are presented in the method section (vide infra - electrochemical measurements). We can see from figure 4.16a that the current density for ORR on $\mathrm{Pt}_{3} \mathrm{Fe} / \mathrm{C}$ electrode is higher than that on $\mathrm{Pt} / \mathrm{C}$ electrode over the entire polarization potential region, which means that the $\mathrm{Pt}_{3} \mathrm{Fe} / \mathrm{C}$ catalyst exhibits a faster electron transfer rate and higher electrocatalytic activity for ORR than $\mathrm{Pt} / \mathrm{C}$. The $\mathrm{Pt}$ mass activities of $\mathrm{Pt}_{3} \mathrm{Fe} / \mathrm{C}$ are $1.02 \mathrm{Amg}_{\mathrm{Pt}}{ }^{-1}$ at $0.85 \mathrm{~V}$, and $0.27 \mathrm{Amg}_{\mathrm{Pt}}{ }^{-1}$ at $0.9 \mathrm{~V}$, which is about 2.5 times higher for the former, indicating that the Pt content could be decreased by 2.5 times in the intermetallic catalyst to obtain the same current density as pure Pt (figure 4.16b). 

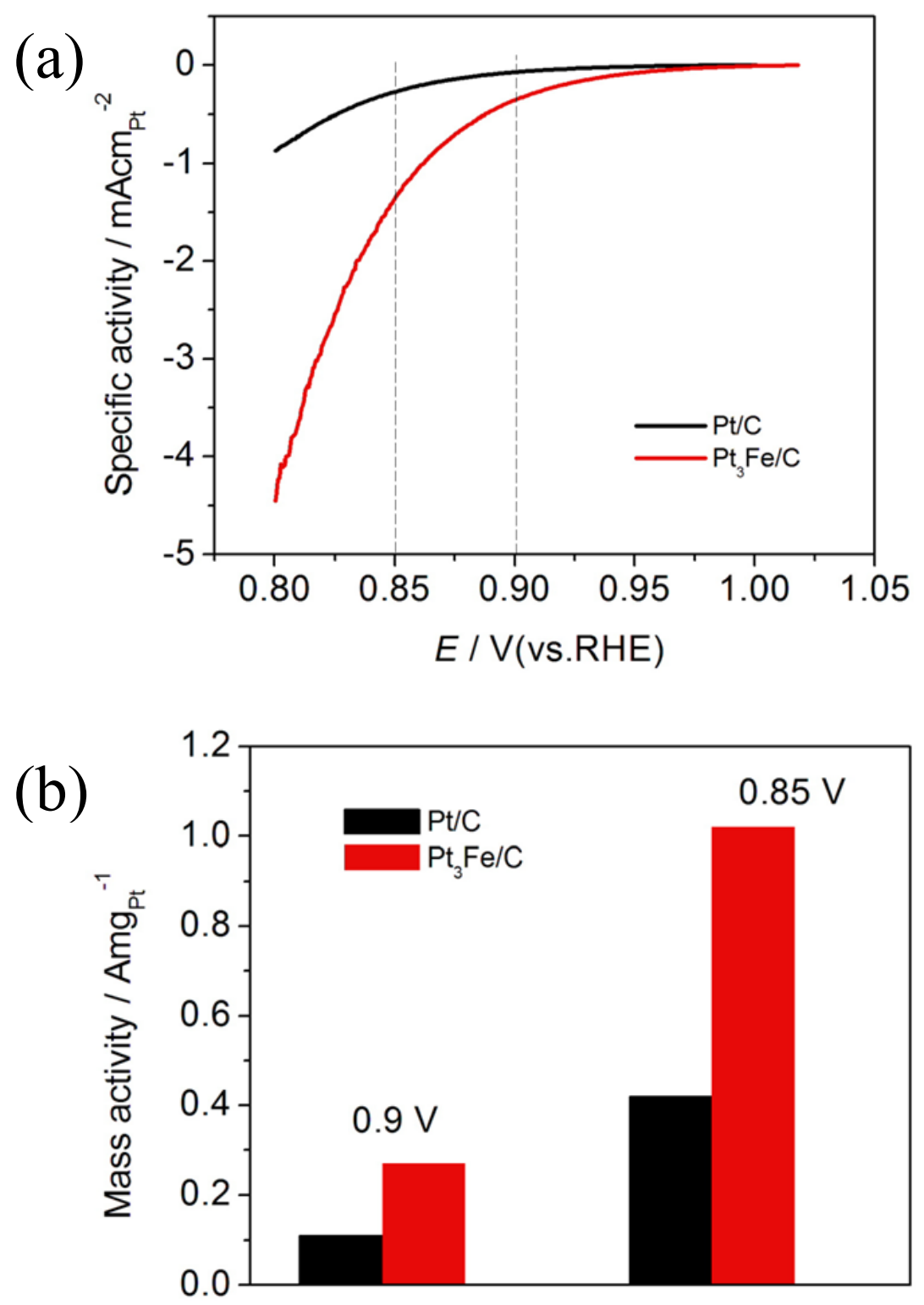

Figure 4.16: (a), The specific activities and (b), The mass activity of the ORR on the two catalysts at $0.9 \mathrm{~V}$ and $0.85 \mathrm{~V}$ obtained from ORR polarization. In all above figures, $\mathrm{Pt}_{3} \mathrm{Fe} / \mathrm{C}$ is in red and $\mathrm{Pt} / \mathrm{C}$ is in black. 


\subsection{Conclusion}
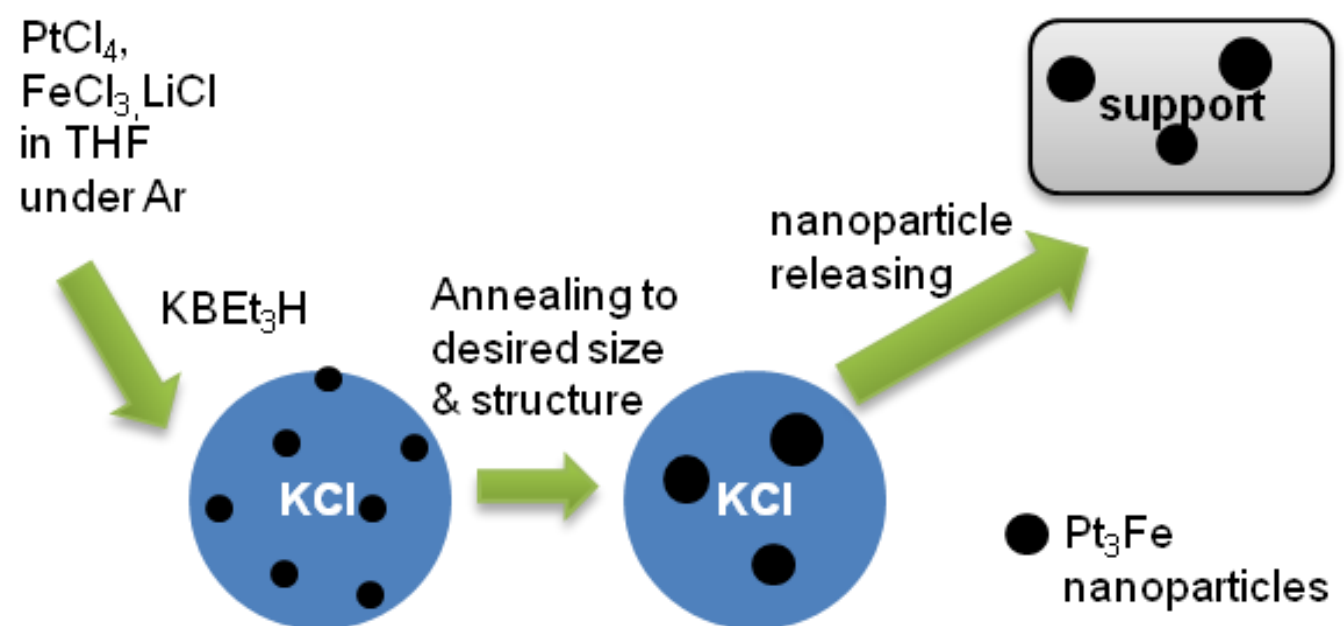

Figure 4.17: Using $\mathrm{Pt}_{3} \mathrm{Fe}$ intermetallic nanoparticles as an example, a scheme for the synthesis and transfer of nanoparticles from a $\mathrm{Np}-\mathrm{KCl}$ matrix to support materials using ethylene glycol was shown to be effective and simple. As described in Chapters $3 \mathrm{Pt}_{3} \mathrm{Fe}$ nanoparticles are formed in a quick room temperature reduction of a THF solution of metal chlorides using $\mathrm{KEt}_{3} \mathrm{BH}$ as the reducing agent. Since the by-product $\mathrm{KCl}$ is not soluble in THF, the newly formed $\mathrm{Pt}_{3} \mathrm{Fe}$ nanoparticles are trapped in the solid $\mathrm{KCl}$. During thermal annealing, nanoparticles grow only very slowly in the $\mathrm{KCl}$ matrix to form somewhat larger particles, while the Pt and Fe atoms order to form an intermetallic compound. After annealing, $\mathrm{Pt}_{3} \mathrm{Fe}$ nanoparticles can be transferred to appropriate supports by dissolving the $\mathrm{KCl}$ salt in ethylene glycol.

As shown in figure 4.17 , chapters 3 and 4 demonstrate a novel strategy to synthesize 
nanoparticles in the desired phase (alloy or ordered) and desired size without using strongly binding organic surfactants.. In this method, $\mathrm{KCl}$ serves as a matrix that traps the nanoparticles to avoid particle agglomeration and to control the coalescence of nanoparticles during thermal annealing. As an example, $\mathrm{Pt}_{3} \mathrm{Fe}$ ordered intermetallic catalysts with average particle sizes around $4 \mathrm{~nm}$ and $2.5 \mathrm{~nm}$ were synthesized by this method. We found that the nanoparticles can be released from the salt in an ethylene glycol solvent and would then uniformly bind to a carbon black support with little agglomeration. Electrochemical studies of the $4 \mathrm{~nm} \mathrm{Pt}_{3} \mathrm{Fe}$ catalyst showed an improved ORR efficiency when compared to pure Pt. The origin of the improved ORR properties is not the focus of this thesis; however, based on our previous results, the ordered intermetallic phase of the core in the resulting core-shell particles appears to play an important role in the improvement.

In the work described in this chapter to develop the process to release the nanoparticles from the $\mathrm{KCl}$ matrix and transfer them to the catalysts support, I received continuous help and support from the current and former members of the DiSalvo group, especially Brian Leonard, Deli Wang and Kathryn Newton, an undergraduate who graduated with her BS in May 2013. The electrochemistry work was carried out collaboratively with Deli Wang. Thank you all! 


\section{REFERENCES}

(1) Dollimore, D; Long, L. H. J Chem Soc 1953, 3906.

(2) Bock, C.; Paquet, C.; Couillard, M.; Botton, G. A.; MacDougall, B. R. J Am Chem Soc 2004, 126, 8028.

(3) Wang, Y.; Ren, J. W.; Deng, K.; Gui, L. L.; Tang, Y. Q. Chem Mater $2000,12,1622$.

(4) Trimble, H. M. Ind Eng Chem 1931, 23, 165.

(5) Skoplyak, O.; Barteau, M. A.; Chen, J. G. G. Surf Sci 2008, 602, 3578.

(6) Skoplyak, O.; Barteau, M. A.; Chen, J. G. Chemsuschem 2008, 1, 524.

(7) Skoplyak, O.; Menning, C. A.; Barteau, M. A.; Chen, J. G. G. Top Catal 2008, 51, 49.

(8) Salciccioli, M.; Yu, W. T.; Barteau, M. A.; Chen, J. G. G.; Vlachos, D. G. J Am Chem Soc 2011, 133, 7996.

(9) Wang, C.; Wang, G. F.; van der Vliet, D.; Chang, K. C.; Markovic, N. M.; Stamenkovic, V. R. Phys Chem Chem Phys 2010, 12, 6933.

(10) Vidal-Iglesias, F. J.; Aran-Ais, R. M.; Solla-Gullon, J.; Herrero, E.; Feliu, J. M. Acs Catal 2012, 2, 901.

(11) Chen, Q. S.; Solla-Gullon, J.; Sun, S. G.; Feliu, J. M. Electrochim Acta 2010, 55, 7982. 


\section{CHAPTER 5}

\section{THE NANOPARTICLE-KCl (Np-KCl) MATRIX METHOD \\ PART III: GENERALITY OF THE METHOD}

\subsection{Introduction}

In chapter 3 and 4 , the methodology of the synthesis of surfactant-free intermetallic nanoparticles using $\mathrm{KCl}$ as a matrix (the $\mathrm{Np}-\mathrm{KCl}$ method) has been demonstrated. In those chapters the $\mathrm{Pt}_{3} \mathrm{Fe}$ and $\mathrm{PtFe}$ systems were examined in detail as examples of the method. The DiSalvo Group (specifically graduate students Minh Nguyen, Doug DeSario and myself, along with a number of undergraduates who have worked with us are currently expanding this method to other intermetallic systems. Our research shows, by using $\mathrm{KBEt}_{3} \mathrm{H}$ as the reducing agent, chloride precursors in periodic table groups 8, 9, 10, 11 and chlorides of elements such as $\mathrm{In}, \mathrm{Sn}, \mathrm{Sb}, \mathrm{Pb}, \mathrm{Bi}$ can be reduced to form Pt-M nanoparticles using the nanoparticle- $\mathrm{KCl}$ matrix method. Using $\mathrm{K}$ naphthalinide as the reducing agent, we have been able to rapidly reduce virtually all metal chlorides to the elemental product in THF solution, including: $\mathrm{Sb}, \mathrm{Bi}, \mathrm{C}, \mathrm{Si}, \mathrm{Ge}$, Sn, B, Al, Ga, In, Zn, Cu, Ag, Au, Ni, Pd, Pt, Co, Fe, Ru, Mn, Cr, Mo, W, V, Nb, Ta, $\mathrm{Ti}, \mathrm{Zr}$, and Hf. Those that are skipped in the later part of the $4 \mathrm{~d}$ and $5 \mathrm{~d}$ transition series no doubt can also be reduced, but we have not had a reason to pursue them.

In this chapter a few examples of the synthesis of other Pt-M intermetallic nanoparticles using the $\mathrm{Np}-\mathrm{KCl}$ matrix method will be discussed, primarily focusing 
on those systems that I developed and on those that could be prepared from alkylborohydrides.

\subsection{Metal hydride formation}

Especially for the synthesis of early transition metals, one of the issues of using organoborohydride as reducing agent is the potential formation of metal hydrides as intermediate or by-products. For example, $\mathrm{ZnCl}_{2}$ in $\mathrm{THF}$ is quickly reduced by $\mathrm{LiEt}_{3} \mathrm{BH}$. The as-reduced powder is in a gray-whitish color. After stirring overnight, the powder transforms to a blackish powder. Such behavior suggests that the firstreduced product is not a metallic material. After 10 or so hours, the color change suggests that $\mathrm{Zn}$ metal is present. Indeed, powder X-ray diffraction confirms that the intital product is $\mathrm{ZnH}_{2}$ and that $\mathrm{Zn}$ metal is the final product after about 10 hours. Since $\mathrm{H}_{2}$ is a by-product of any borohydride reduction, the possibility of hydride formation must be considered. The formation of a metastable or stable hydride intermediates during reduction will likely impede the formation of homogeneous alloy or ordered intermetallic nanoparticles.

As shown in figure 5.1, many metals are expected to form hydrides at room temperature at one atmosphere of hydrogen pressure. However, late transition metals like $\mathrm{Fe}, \mathrm{Co}$, and $\mathrm{Cu}$ do not form a stable or even a metastable hydrides under the above conditions $^{1,2}$. Given the rather large heats of formation of most Pt-M, such metal 
hydride byproducts of the late transition metals should not be the lowest free energy products of the overall reaction. The question becomes one of kinetics if hydride intermediates are indeed produced. The enthalpy of hydride formation for the early transition metals is quite negative and needs to be taken into consideration. For example, the enthalpy of formation of $\mathrm{TiH}_{2}$ is $-142.4 \mathrm{~kJ} / \mathrm{mol}^{3,4}$, of $\mathrm{VH}_{2}$ is -76.2 $\mathrm{kJ} / \mathrm{mol}^{4}$, of $\mathrm{MnH}$ is $-50.0 \mathrm{~kJ} / \mathrm{mol}^{5}$, and of $\mathrm{ZrH}_{2}$ is $-162.8 \mathrm{~kJ} / \mathrm{mol}^{6}$. A summary of the enthalpies of some of the early transition metals hydrides are given in figure 5.2. The thermodymanic stability of the hydrides when reacting with $\mathrm{Pt}$ is temperature dependent and the reaction to form intermetallic phases becomes spontaneous above room temperature. Thus thermal annealing should produce the desired products. It is interesting to ask if this will be the case when $\mathrm{KCl}$ is produced as a by-product and the solid products are trapped in the $\mathrm{KCl}$. During the thermal annealing process, the following equation can be considered for the alloying process of Pt with, for example, $\mathrm{TiH}_{2}$ (equation 5.1):

$$
\mathrm{TiH}_{2}(\mathrm{~s})+\operatorname{Pt}(\mathrm{s}) \leftrightarrow \operatorname{PtTi}(\mathrm{s})+\mathrm{H}_{2}(\mathrm{~g}) \quad \text { (Equation 5.1, } \mathrm{s}-\text { solid, } \mathrm{g}=\text { gas) }
$$

The standard enthalpies of formation $\left(\mathrm{H}_{\mathrm{f}}^{\mathrm{o}}\right)$ of the solids are all negative, but the change in entropy on reaction is positive due to the formation of a gaseous product, $\mathrm{H}_{2}$. The free energy $(\Delta \mathrm{G})$ of the forward reaction can then be estimated versus temperature (T) as below (equation 5.2, again using $\mathrm{TiH}_{2}$ as an example):

$$
\Delta \mathrm{G}=\left(\mathrm{H}_{(\mathrm{PtTi})}-\mathrm{H}_{(\mathrm{TiH} 2)}\right)-\mathrm{S}_{(\mathrm{H} 2)} * \mathrm{~T} \quad \text { (Equation 5.2) }
$$




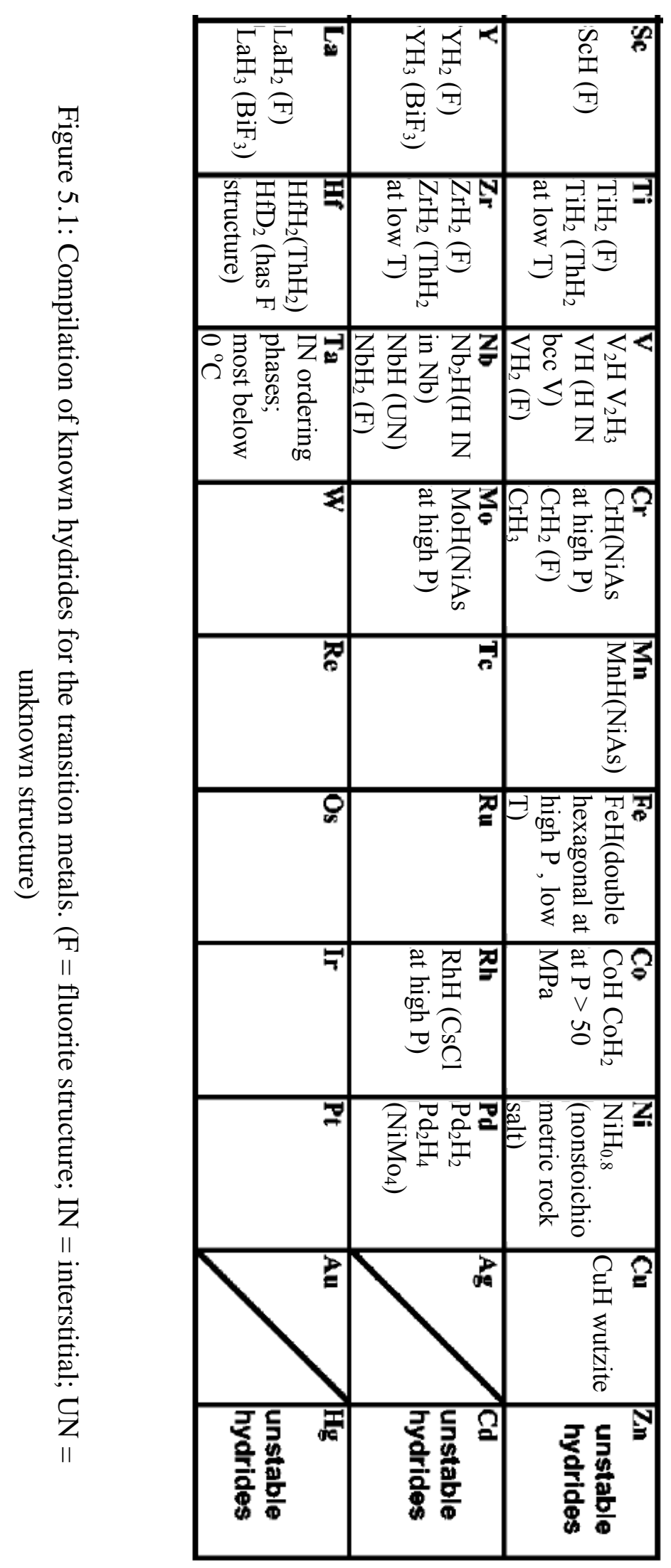


Equation 5.2 assumes that the difference in enthalpy is only weakly temperature dependent and that the temperature dependence can be ignored. Thus the difference in enthalpy equals the difference in free energy at $\mathrm{T}=0 \mathrm{~K}$.

At a certain temperature, if $\Delta \mathrm{G}$ becomes negative, the equilibrium will move toward the alloying product direction and the PtM alloy will be formed releasing $\mathrm{H}_{2}$ gas. Given that the enthalpy of formation of $\mathrm{TiH}_{2}$ is $-142.4 \mathrm{~kJ} / \mathrm{mol}^{3,4}$ and the enthalpy of formation of PtTi is $-91.0 \mathrm{~kJ} / \mathrm{mol}^{7}$, the free energy of this process will become negative above approximately $390 \mathrm{~K}$. This temperature is way below typical annealing temperatures $\left(400\right.$ to $600{ }^{\circ} \mathrm{C}$ ) to obtain ordered Pt-Ti products. This assumes that the reactant hydride and Pt nano-particles are either already aggregated, as would likely be the case using $\mathrm{Li}$ alkylborohydride reducing agent, or can diffuse together through the $\mathrm{KCl}$ matrix when $\mathrm{K}$ alkylborohydride is used. Similarly, the temperature where the free energy favors the alloying reaction of $\mathrm{Pt}$ and other transition metals can be calculated assuming they started with the metal hydride phases. The detailed calculated results are shown in figure 5.2. As can be seen, for all of the early transition metals of interest, such temperatures are below typical annealing temperatures. 


\begin{tabular}{|c|c|c|c|c|c|}
\hline Pt-M & Reaction & $\begin{array}{l}\Delta \mathrm{H} \mathrm{MH} \\
\mathrm{kJ} \mathrm{mol}^{-1}\end{array}$ & $\begin{array}{l}\Delta \mathrm{H} \mathrm{Pt}_{\mathbf{x}} \mathrm{M} \\
\mathrm{kJ} \mathrm{mol}^{-1}\end{array}$ & $\begin{array}{l}\Delta \mathrm{S}\left(\mathbf{H}_{2}\right) \\
\mathbf{J} \mathbf{m o l}^{-1} \mathbf{K}^{-1}\end{array}$ & $\begin{array}{l}\mathbf{T}(\mathbf{K}) \\
\Delta \mathbf{G}=\mathbf{0}\end{array}$ \\
\hline $\mathrm{Pt}_{3} \mathrm{Ti}$ & $\begin{aligned} & 3 \mathrm{Pt}(\mathrm{s})+\mathrm{TiH}_{2} \rightarrow \mathrm{Pt}_{3} \mathrm{Ti} \\
+ & \mathrm{H}_{2}\end{aligned}$ & $-142.4^{3,4}$ & $-81.56^{8}$ & 130 & 467 \\
\hline PtTi & $\mathrm{Pt}(\mathrm{s})+\mathrm{TiH}_{2} \rightarrow \mathrm{PtTi}+\mathrm{H}_{2}$ & -142.39 & $-91^{\prime}$ & 130 & 392 \\
\hline $\mathrm{Pt}_{3} \mathrm{~V}$ & $\begin{array}{l}3 \mathrm{Pt}(\mathrm{s})+\mathrm{VH}_{2} \rightarrow \mathrm{Pt}_{3} \mathrm{~V} \\
+\mathrm{H}_{2}\end{array}$ & $-76.2^{4}$ & $-30.4^{y}$ & 130 & 352 \\
\hline $\mathrm{PtV}$ & $\mathrm{Pt}(\mathrm{s})+\mathrm{VH}_{2} \rightarrow \mathrm{PtV}+\mathrm{H}_{2}$ & -76.2 & $-45.3^{10}$ & 130 & 237 \\
\hline $\mathrm{Pt}_{3} \mathrm{Cr}$ & $\begin{array}{l}3 \mathrm{Pt}(\mathrm{s})+\mathrm{CrH} \rightarrow \mathrm{Pt}_{3} \mathrm{Cr}+ \\
1 / 2 \mathrm{H}_{2}\end{array}$ & $-7.07^{9}$ & $-8.8^{11}$ & 65 & $* * *$ \\
\hline PtMn & $\begin{array}{l}\mathrm{Pt}(\mathrm{s})+\mathrm{MnH} \rightarrow \mathrm{PtMn} \\
+1 / 2 \mathrm{H}_{2}\end{array}$ & $-50^{3}$ & $-53.06^{11}$ & 65 & $* * *$ \\
\hline PtZr & $\mathrm{Pt}(\mathrm{s})+\mathrm{ZrH}_{2} \rightarrow \mathrm{PtZr}+\mathrm{H}_{2}$ & $-162.79^{6}$ & $-104.1^{7}$ & 130 & 451 \\
\hline $\mathrm{Pt}_{3} \mathrm{Zr}$ & $\begin{aligned} & 3 \mathrm{Pt}(\mathrm{s})+\mathrm{ZrH}_{2} \rightarrow \mathrm{Pt}_{3} \mathrm{Zr} \\
& +\mathrm{H}_{2}\end{aligned}$ & -162.79 & $-119^{9,12}$ & 130 & 444 \\
\hline $\mathrm{PtNb}$ & $\begin{array}{l}\mathrm{Pt}(\mathrm{s})+\mathrm{NbH}_{2} \rightarrow \mathrm{PtNb} \\
+\mathrm{H}_{2}\end{array}$ & $\sim-115^{3}$ & $-85^{y, 10}$ & 130 & 230 \\
\hline $\mathrm{Pt}_{3} \mathrm{Nb}$ & $\begin{array}{l}3 \mathrm{Pt}(\mathrm{s})+\mathrm{NbH}_{2} \rightarrow \mathrm{Pt}_{3} \mathrm{Nb} \\
+\mathrm{H}_{2}\end{array}$ & $\sim-115$ & $-46.6^{y}$ & 130 & 526 \\
\hline
\end{tabular}




\begin{tabular}{|c|c|c|c|c|c|}
\hline Pt-M & Reaction & $\begin{array}{l}\Delta \mathrm{H} \mathrm{MH}_{\mathrm{x}} \\
\mathbf{k J} \mathrm{mol}^{-1}\end{array}$ & $\begin{array}{l}\Delta \mathrm{H} \mathrm{Pt} \mathbf{x}_{\mathbf{x}} \mathrm{M} \\
\mathrm{kJ} \mathrm{mol}^{-1}\end{array}$ & $\begin{array}{l}\Delta S\left(\mathbf{H}_{2}\right) \\
\mathrm{J} \mathrm{mol}^{-1} \mathbf{K}^{-1}\end{array}$ & $\begin{array}{l}\mathbf{T}(\mathbf{K}) \\
\Delta \mathbf{G}=\mathbf{0}\end{array}$ \\
\hline PtHf & $\begin{array}{l}\mathrm{Pt}(\mathrm{s})+\mathrm{HfH}_{2} \rightarrow \mathrm{PtHf} \\
+\mathrm{H}_{2}\end{array}$ & $-142.0^{13}$ & $-158.3^{10}$ & & $* * *$ \\
\hline $\mathrm{Pt}_{3} \mathrm{Hf}$ & $\begin{array}{l}\mathrm{Pt}(\mathrm{s})+\mathrm{HfH}_{2} \rightarrow \mathrm{Pt}_{3} \mathrm{Hf} \\
+\mathrm{H}_{2}\end{array}$ & -142.0 & $-137.9^{12}$ & 130 & 31 \\
\hline PtTa & $\begin{array}{l}\mathrm{Pt}(\mathrm{s})+\mathrm{TaH}_{0.5} \rightarrow \mathrm{PtTa} \\
+1 / 4 \mathrm{H}_{2}\end{array}$ & $-19.24^{14}$ & $-96.5^{10}$ & & $* * *$ \\
\hline $\mathrm{Pt}_{3} \mathrm{Ta}$ & $\begin{array}{l}\mathrm{Pt}(\mathrm{s})+\mathrm{TaH}_{0.5} \rightarrow \mathrm{Pt}_{3} \mathrm{Ta} \\
+1 / 4 \mathrm{H}_{2}\end{array}$ & -19.24 & $-21.1^{11}$ & & $* * *$ \\
\hline
\end{tabular}

Figure 5.2: Calculated transition temperatures for alloying some early transition metals with Pt from their hydride form. The "***" in temperature column means the alloying enthalpy is higher than the enthalpy of the metal hydrides at room temperature. 


\subsection{The synthesis of $\mathrm{Pt}_{3} \mathrm{Mn}$ intermetallic nanoparticles}

$\mathrm{Li}_{2} \mathrm{MnCl}_{4}$ was synthesized according to the phase diagram by heating $\mathrm{LiCl}$ and $\mathrm{MnCl}_{2}$ (with a mole ratio of 2:1) in a sealed silica tube under vacuum for 24 hours at $600{ }^{\circ} \mathrm{C}$.

The synthesis of $\mathrm{Pt}_{3} \mathrm{Mn}-80 \mathrm{KCl}$ was carried as follows: $0.24 \mathrm{mmol} \mathrm{PtCl}_{4}, 0.08 \mathrm{mmol}$ $\mathrm{Li}_{2} \mathrm{MnCl}_{4}$ and $5.2 \mathrm{mmol} \mathrm{LiCl}$ were weighed out in an argon filled glovebox and dissolved in $20 \mathrm{~mL}$ THF by stirring. $7 \mathrm{~mL} \mathrm{KEt}_{3} \mathrm{BH}$ (1M Sigma-Aldrich) was mixed with THF to form a $10 \mathrm{~mL}$ solution. Then the precursor solution was drawn up into a syringe and injected into the borohydride reducing agent solution under vigorous stirring. The sample was dried under vacuum until most of the THF was evaporated. Hexanes were then added to precipitate all the $\mathrm{Np}-\mathrm{KCl}$ powders. The sample was subsequently washed three times with mixed solution of THF and hexanes without contacting air. The product was then dried under vacuum for 2 hours and transferred to the Ar glovebox. The $\mathrm{Pt}_{3} \mathrm{Mn}-80 \mathrm{KCl}$ powder was then placed into silica tubes, which were then transferred to a Schlenk / vacuum line and sealed under vacuum. The sample was then heated to $545{ }^{\circ} \mathrm{C}$ at a rate of $100{ }^{\circ} \mathrm{C}$ / hour and annealed at $545{ }^{\circ} \mathrm{C}$ for 6 hours and cooled to $300{ }^{\circ} \mathrm{C}$ at $30^{\circ} \mathrm{C}$ / hour and then cooled to room temperature by shutting off the furnace power. The samples were then transferred to Vulcan carbon support using ethylene glycol as transfer agent.

The XRD, STEM, EDX and STEM-EDX line scans were then conducted to demonstrate the intermetallic structure. 


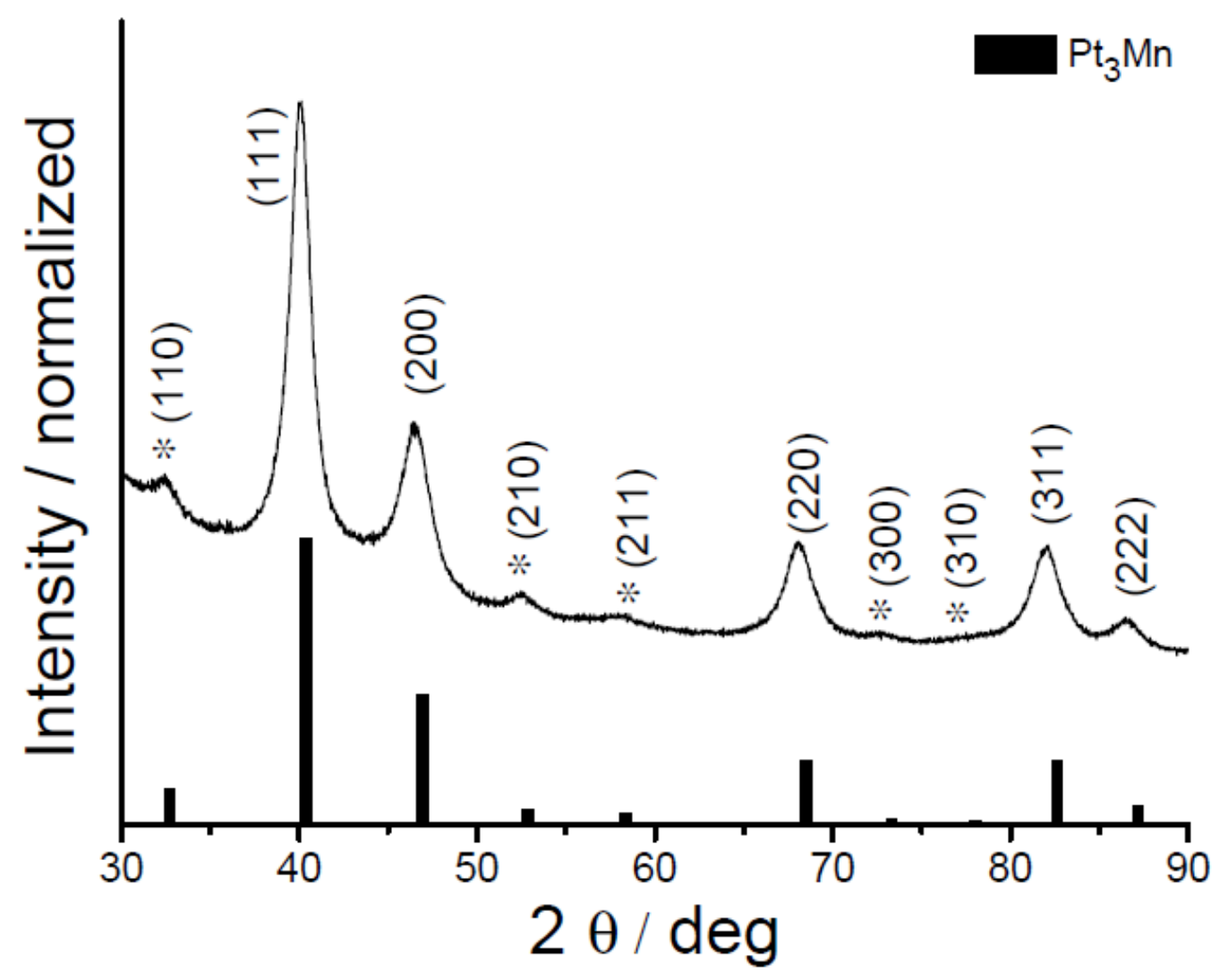

Figure 5.3: XRD spectra of carbon supported $\mathrm{Pt}_{3} \mathrm{Mn}$, The lines on the bottom indicate the XRD peak positions of the corresponding intermetallic phase of the $\mathrm{Pt}_{3} \mathrm{Mn}$ (PDF card \# 04-002-1438). The peak positions for the ordered intermetallic phase of $\mathrm{Pt}_{3} \mathrm{Mn}$ are marked with *.

The XRD of the sample transferred to Vulcan is shown in figure 5.3. The only peaks observed are indexed by the ordered cubic structure of $\mathrm{Pt}_{3} \mathrm{Mn}$. No peaks corresponding 
to $\mathrm{Pt}, \mathrm{Mn}$, other intermetallics in the Pt-Mn phase diagram (such as the 1:1 PtMn intermetallic phase), or any other Pt- or Mn-containing compounds are present in the diffraction pattern. The observation of ordering peaks, such as the expected weak (110), (210) and (211) peaks, demonstrates that the desired ordered intermetallic phase rather than the disordered alloy phase of Pt-Mn was prepared.

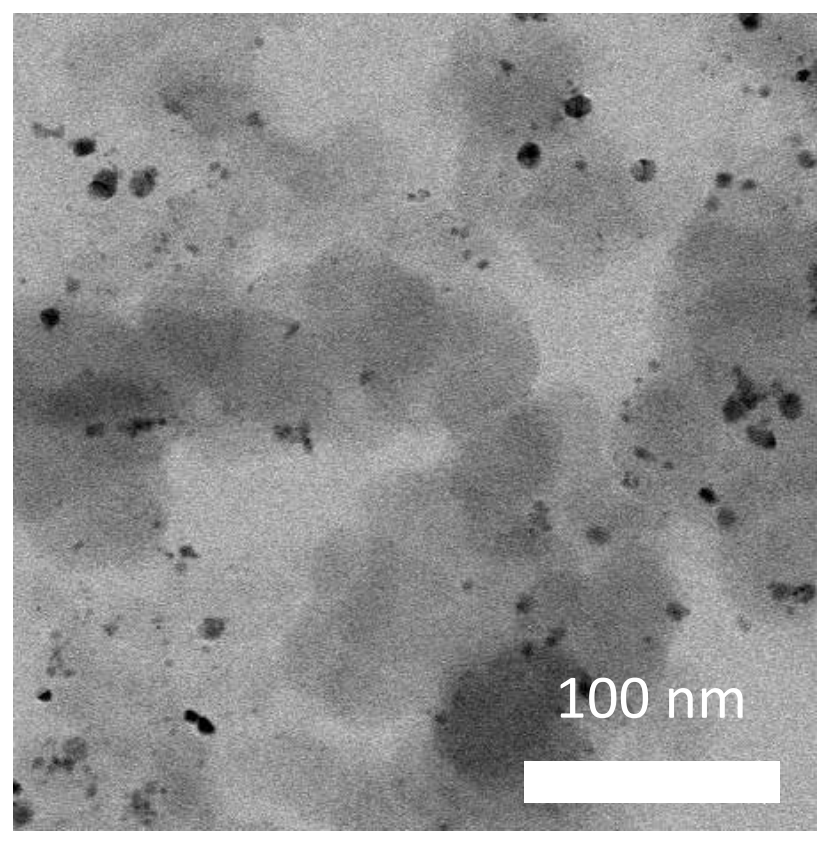

Figure 5.4: STEM-BF image of the annealed $\mathrm{Pt}_{3} \mathrm{Mn}$ supported on carbon black

Shown in figure 5.4 is the STEM-BF image of the annealed $\mathrm{Pt}_{3} \mathrm{Mn}$ supported on carbon black. Clearly, the particles are distributed on the carbon support without 
obvious aggregation and as expected the particle sizes are mostly between 5 and 10 $\mathrm{nm}$. The target loading was $10 \mathrm{wt} \%$.

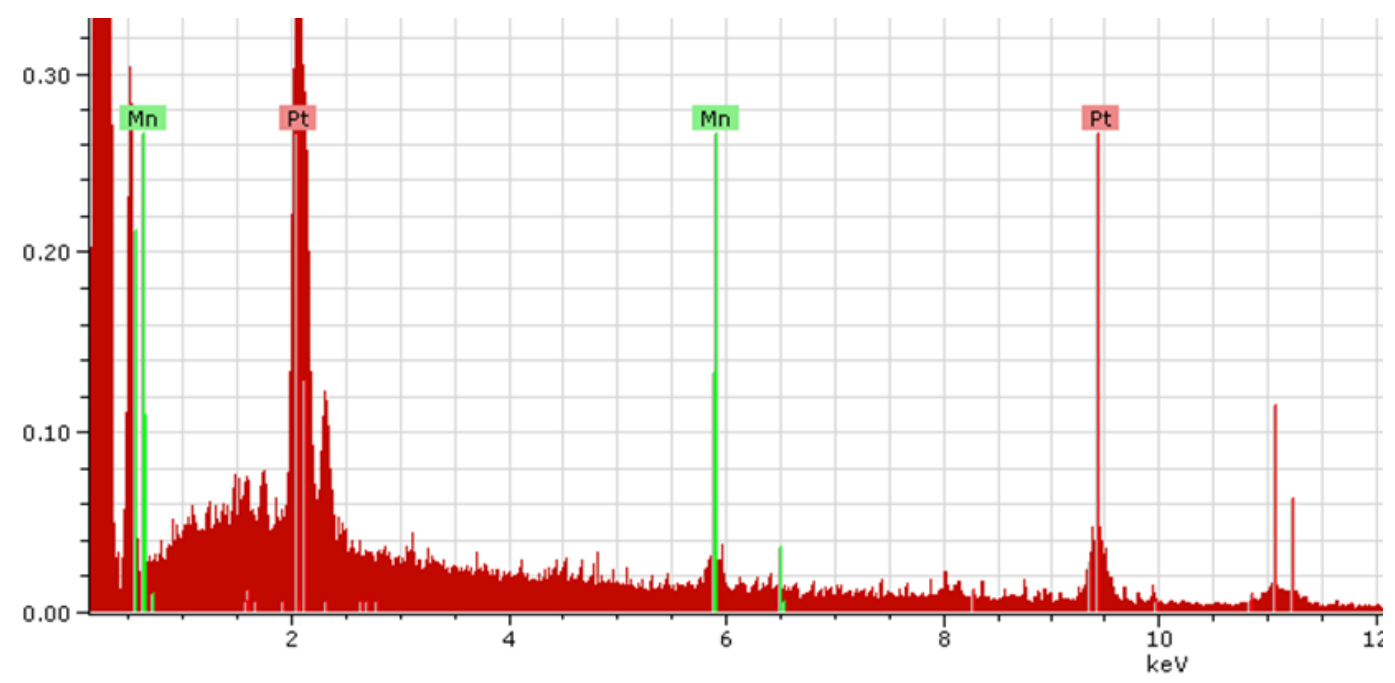

Figure 5.5: The EDX spectrum of $\mathrm{Pt}_{3} \mathrm{Mn}$ sample

The average composition of an ensemble of nanoparticles in an area of $500 \mathrm{~nm}$ by 500 nm obtained by EDX spectroscopy for Pt:Mn was typically 75:25 $( \pm 3 \%)$ and uniform throughout the sample (figure 5.5). STEM-EDX line scans of the $\mathrm{Pt}_{3} \mathrm{Mn}$ nanoparticle confirms that the elements are distributed uniformly in all the examined nanoparticles (figure 5.6). 

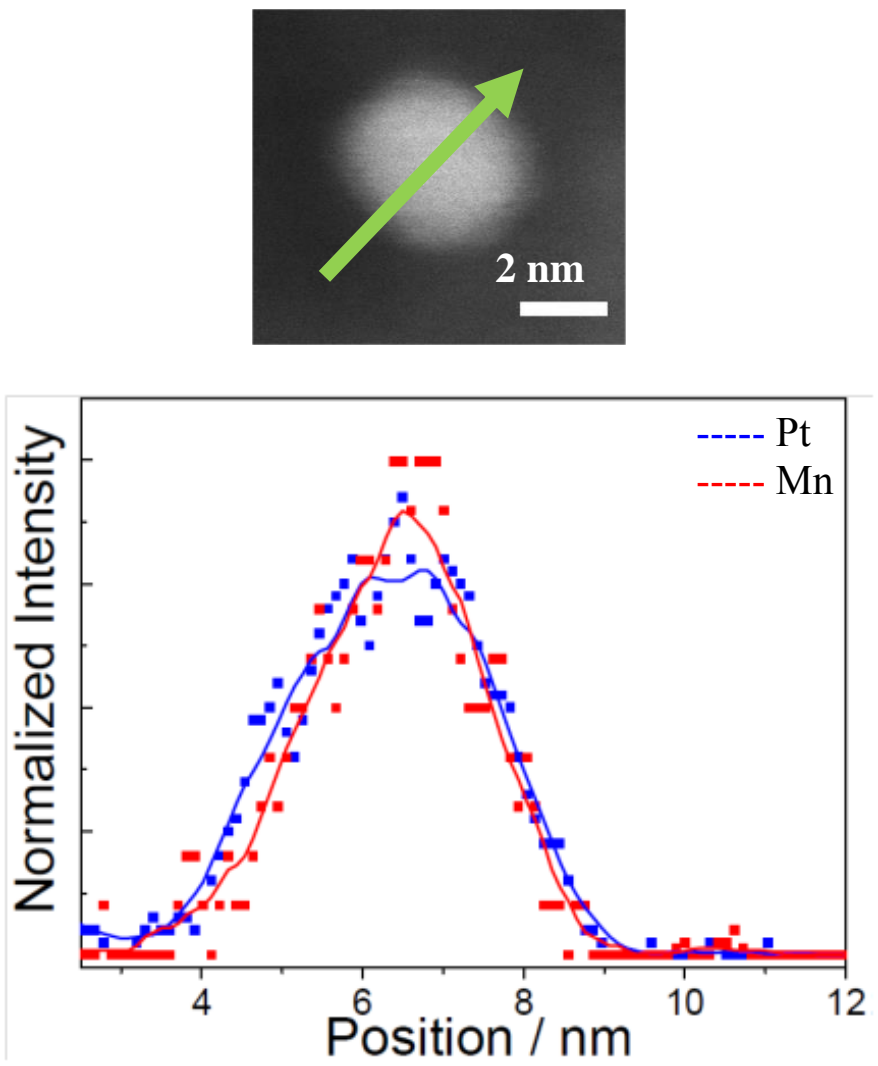

Figure 5.6: STEM-EDX line scans of $\mathrm{Pt}_{3} \mathrm{Mn}$ nanoparticle show that the elements are distributed uniformly in the particles. The best-fit lines are extrapolated by averaging adjacent points.

All measurements, including XRD, STEM, EDX and STEM-EDX line scans, confirm that the ordered intermetallic $\mathrm{Pt}_{3} \mathrm{Mn}$ can be synthesized and transferred to support materials using the $\mathrm{Np}-\mathrm{KCl}$ method. 


\subsection{The synthesis of PtNi intermetallic nanoparticles}

$\mathrm{Li}_{6} \mathrm{NiCl}_{8}$ was synthesized according to the phase diagram by heating $\mathrm{LiCl}$ and $\mathrm{NiCl}_{2}$ (with a mole ratio of 6:1) in a sealed silica tube under vacuum for 15 days at $525{ }^{\circ} \mathrm{C}$. The $\mathrm{Li}_{6} \mathrm{NiCl}_{8}$ can be readily dissolved in THF resulting in a bright blue solution, whereas $\mathrm{NiCl}_{2}$ is quite insoluble in THF.

The synthesis of PtNi-40KCl was carried out as follows: $0.08 \mathrm{mmol} \mathrm{PtCl}_{4}, 0.08 \mathrm{mmol}$ $\mathrm{Li}_{6} \mathrm{NiCl}_{8}$ and $4.48 \mathrm{mmol} \mathrm{LiCl}$ were weighed out in an argon filled glovebox and dissolved in $20 \mathrm{~mL}$ THF by stirring. $7 \mathrm{~mL} \mathrm{KEt}_{3} \mathrm{BH}$ (1M Sigma-Aldrich) were mixed with THF to form a $10 \mathrm{~mL}$ solution. Then the precursor solution was drawn up into a syringe and injected into the reducing agent solution under vigorous stirring. The sample was dried under vacuum until most of the THF was evaporated. Hexanes were then added to precipitate the $\mathrm{Np}-\mathrm{KCl}$ powders. The sample was subsequently washed three times with a mixtured solution of THF and hexanes without contacting air. The product was then dried under vacuum for 2 hours and transferred into the Ar glovebox. The PtNi-40KCl powder was then placed into silica tubes, which were then transferred to a Schlenk / vacuum line and sealed under vacuum. The sample was then heated to $600{ }^{\circ} \mathrm{C}$ at a rate of $100{ }^{\circ} \mathrm{C}$ / hour and annealed at $600{ }^{\circ} \mathrm{C}$ for 6 hours and cooled to 300 ${ }^{\circ} \mathrm{C}$ at $30^{\circ} \mathrm{C} /$ hour and then cooled to room temperature. The samples were then transferred to Vulcan carbon support using ethylene glycol as transfer agent. 


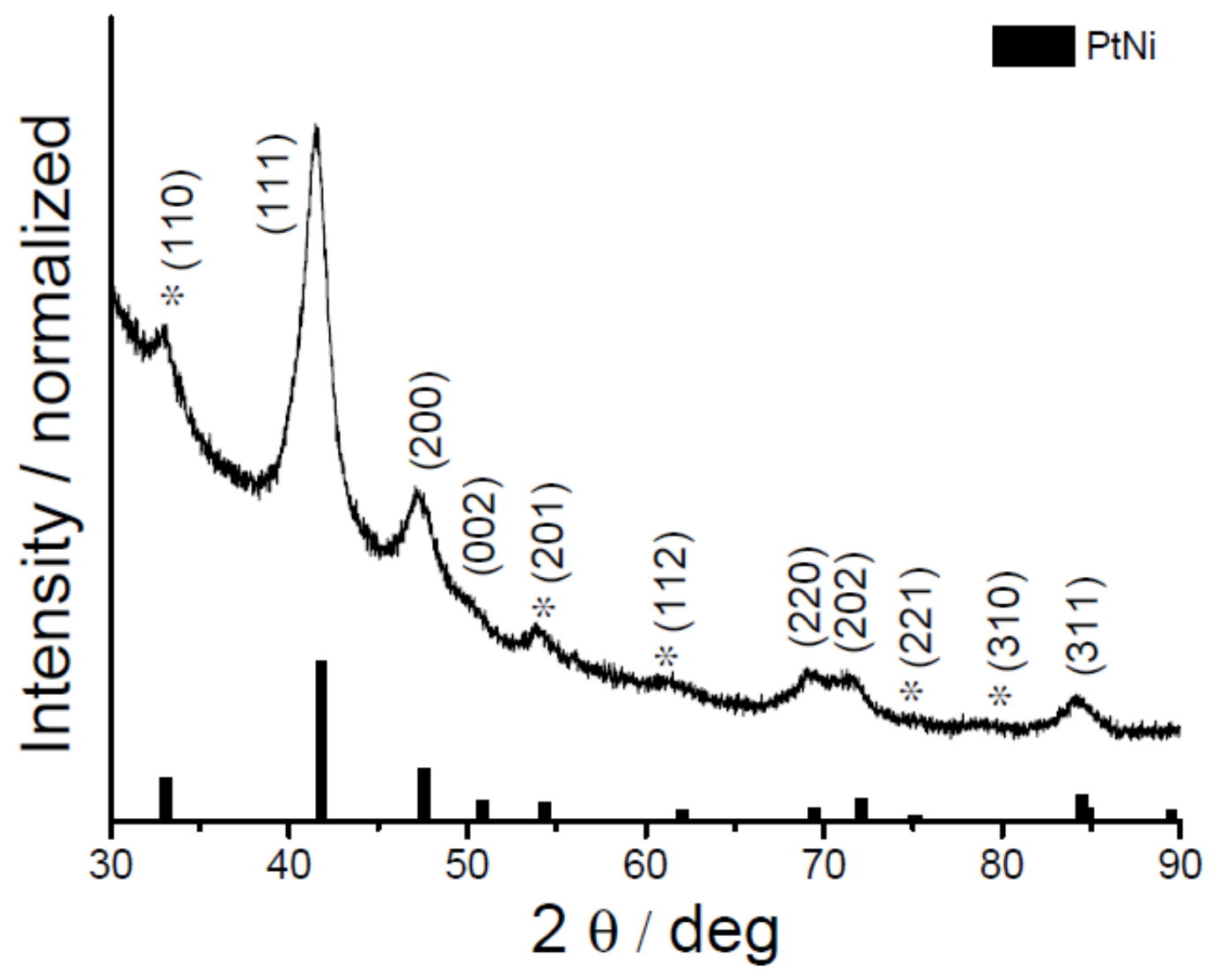

Figure 5.7: XRD spectra of carbon supported PtNi, The lines on the bottom indicate the XRD peak positions of the corresponding intermetallic phase of the PtNi (PDF card \#03-065-2797) The peak positions for the ordered intermetallic phase of PtNi are marked with *. The large sloping background in the pattern is due to the presence of Vulcan. 
The XRD of the transferred sample is shown in figure 5.7. The only peaks observed are indexed by the ordered tetragonal structure of PtNi. No peaks corresponding to Pt, $\mathrm{Ni}$, and other intermetallics in the Pt-Ni phase diagram or any other Pt- or Nicontaining compounds are present in the diffraction pattern. The observation of ordering peaks, such as the expected weak (110), (201) and (112) peaks, demonstrates that the desired ordered intermetallic phase rather than the disordered alloy phase of Pt-Ni was prepared.

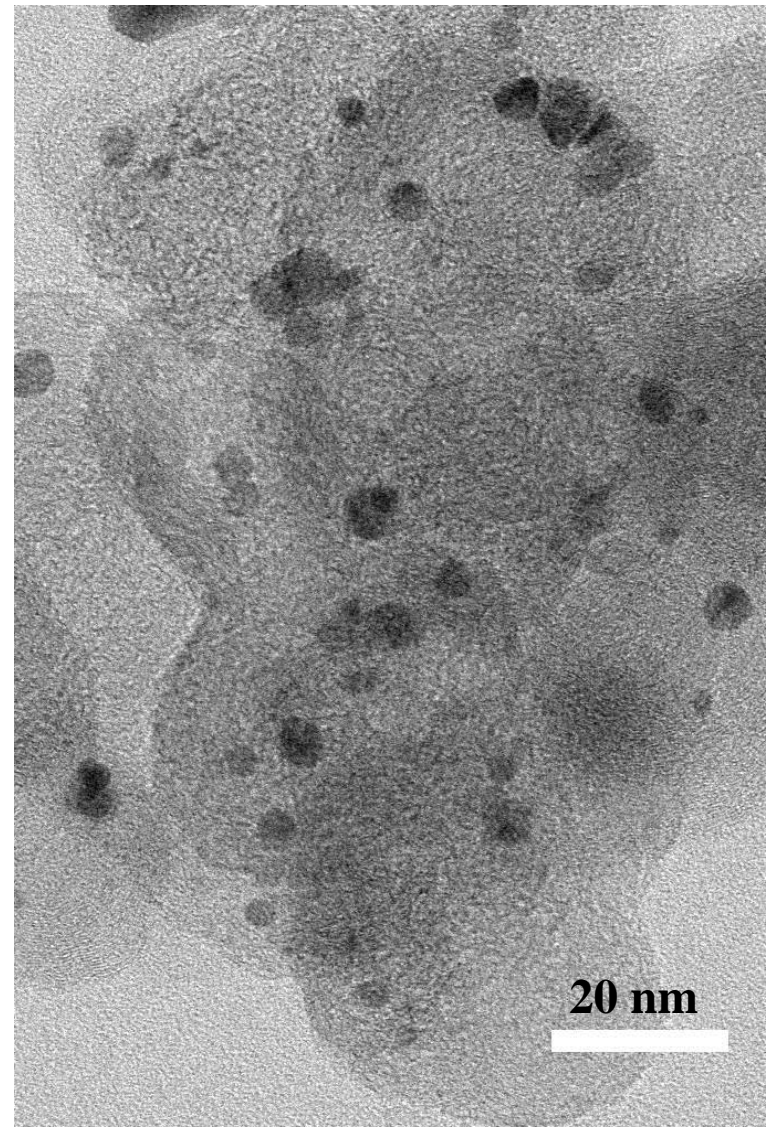

Figure 5.8: STEM-BF image of the annealed PtNi supported on carbon black 
Shown in figure 5.8 is the STEM-BF image of the annealed PtNi supported on carbon black. Clearly, the particles are distributed on the carbon support without obvious aggregation and the particle sizes are small, typically between 3 and $6 \mathrm{~nm}$..

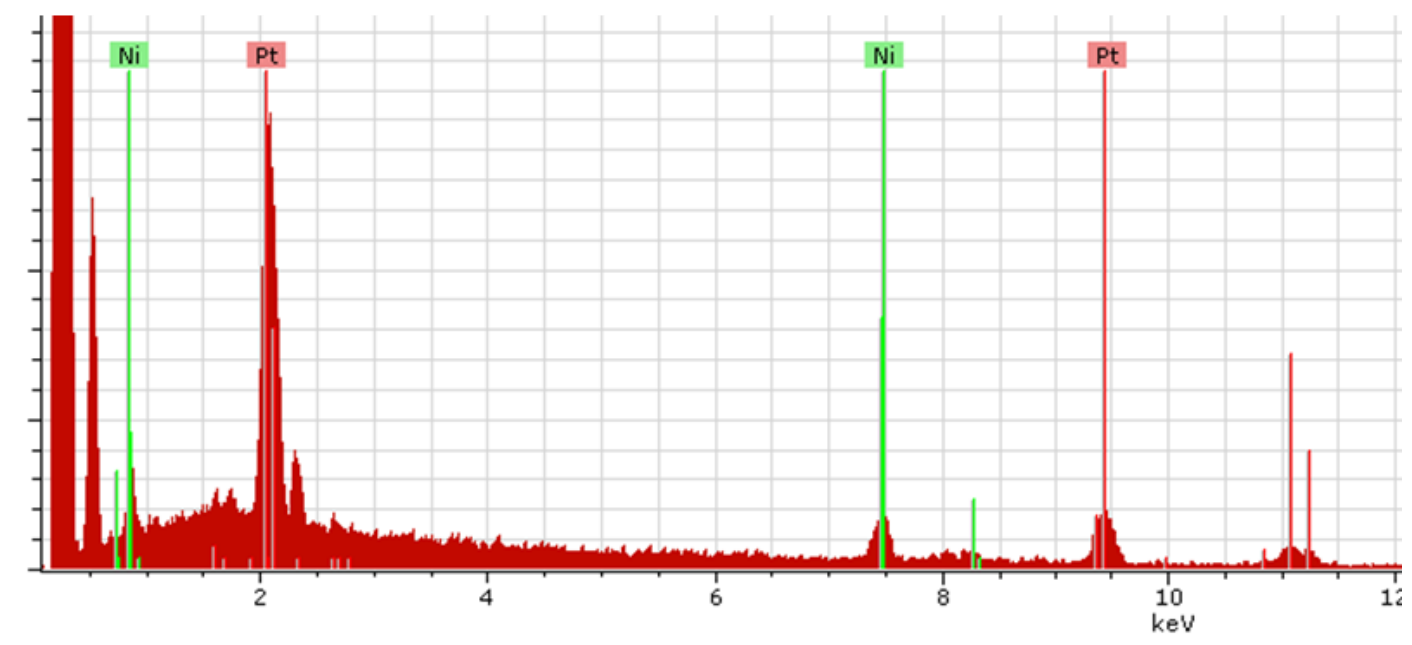

Figure 5.9: The EDX spectrum of PtNi sample

The average composition of an ensemble of nanoparticles in an area of $500 \mathrm{~nm}$ by 500 nm obtained by EDX spectroscopy for Pt:Ni was typically 50:50 $( \pm 3 \%)$ and uniform throughout the sample (figure 5.9). STEM-EDX line scans of the PtNi nanoparticle confirms that the elements are distributed uniformly in all the examined nanoparticles (figure 5.10). 

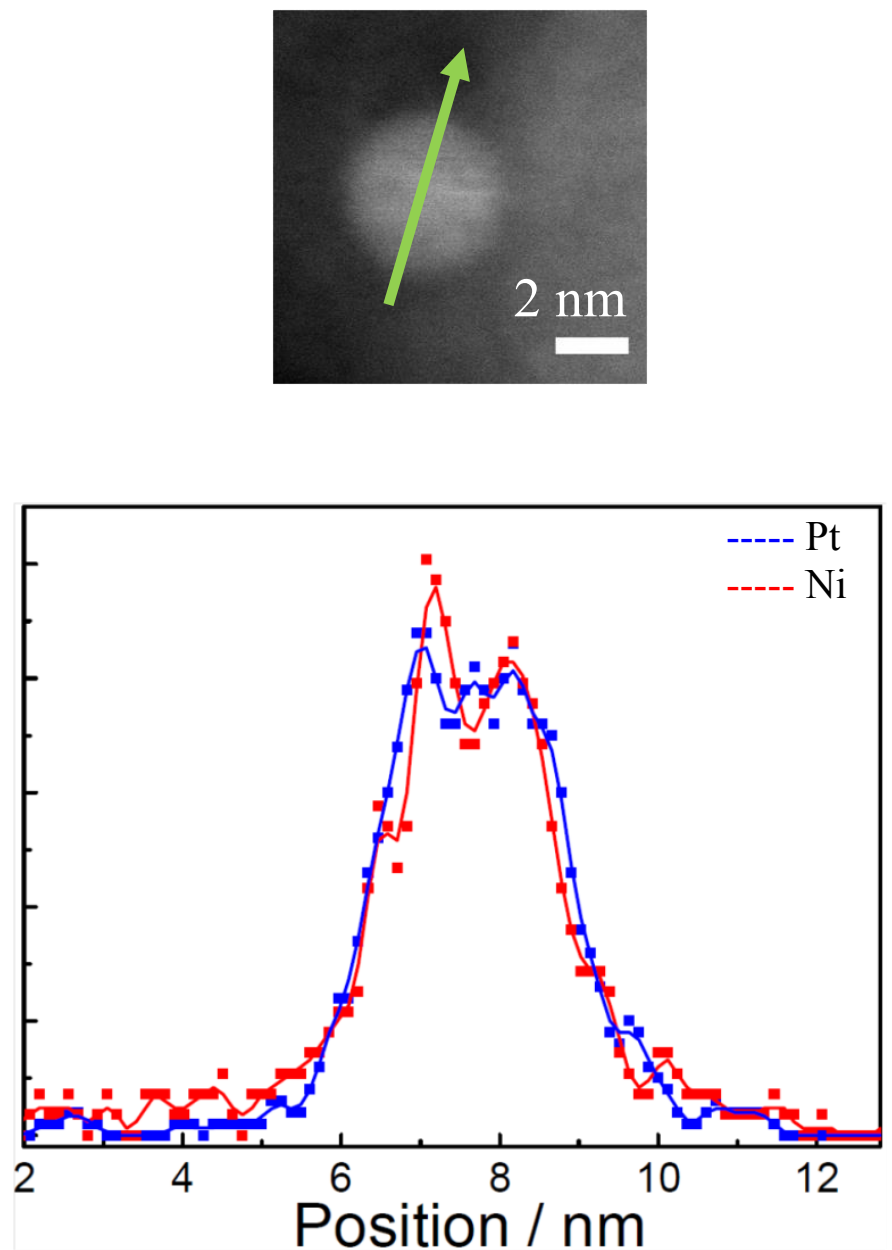

Figure 5.10: STEM-EDX line scans of PtNi nanoparticle show that the elements are distributed uniformly in the particles. The best-fit lines are extrapolated by averaging adjacent points.

All measurements, including XRD, STEM, EDX and STEM-EDX line scans, confirm that the ordered intermetallic PtNi has been synthesized and transferred to support materials. 


\subsection{The synthesis of $\mathrm{Pt}_{2} \mathrm{CoFe}$ intermetallic nanoparticles}

$\mathrm{Li}_{2} \mathrm{CoCl}_{4}$ has been studied spectroscopically in several molten salts, and it was hypothesized that the polarizing power of the small $\mathrm{Li}^{+}$ion would help pull the tetrahedral $\mathrm{CoCl} 4^{2-}$ ions into solution. $\mathrm{Li}_{2} \mathrm{CoCl}_{4}$ was synthesized according to the phase diagram by heating $\mathrm{LiCl}$ and $\mathrm{CoCl}_{2}$ (with a mole ratio of 2:1) in a sealed silica tube under vacuum for 7 days at $400{ }^{\circ} \mathrm{C}$. The $\mathrm{Li}_{2} \mathrm{CoCl}_{4}$ can be readily dissolved in THF resulting in a bright green solution, whereas $\mathrm{CoCl}_{2}$ is quite insoluble in THF.

The synthesis of $\mathrm{Pt}_{2} \mathrm{FeCo}-60 \mathrm{KCl}$ was carried as follows: $0.16 \mathrm{mmol} \mathrm{PtCl}_{4}, 0.08 \mathrm{mmol}$ $\mathrm{FeCl}_{3}, 0.08 \mathrm{mmol} \mathrm{Li}_{2} \mathrm{CoCl}_{4}$ and $8.4 \mathrm{mmol} \mathrm{LiCl}$ were weighed out in an argon filled glovebox and dissolved in $20 \mathrm{~mL}$ THF by stirring. $10 \mathrm{~mL}$ of $\mathrm{KEt}_{3} \mathrm{BH}$ (1M SigmaAldrich) was used as the reducing agent. The precursor solution was drawn up into a syringe and injected into the reducing agent solution under vigorous stirring. The sample was dried under vacuum until most of the THF was evaporated. Hexanes were then added to precipitate the $\mathrm{Np}-\mathrm{KCl}$ powders. The sample was subsequently washed three times with a mixture solution of THF and hexanes without contacting air. The product was then dried under vacuum for 2 hours and transferred to the Ar glovebox. The $\mathrm{Pt}_{2} \mathrm{FeCo}-60 \mathrm{KCl}$ powder was then placed into silica tubes, which were then transferred to a Schlenk / vacuum line and sealed under vacuum. The sample was then heated to $600{ }^{\circ} \mathrm{C}$ at a rate of $100{ }^{\circ} \mathrm{C} /$ hour and annealed at $600{ }^{\circ} \mathrm{C}$ for 6 hours and cooled to $300{ }^{\circ} \mathrm{C}$ at $30^{\circ} \mathrm{C}$ / hour and then cooled to room temperature by turning off the furnace power. The samples were then transferred to Vulcan carbon support using ethylene glycol as transfer agent. 


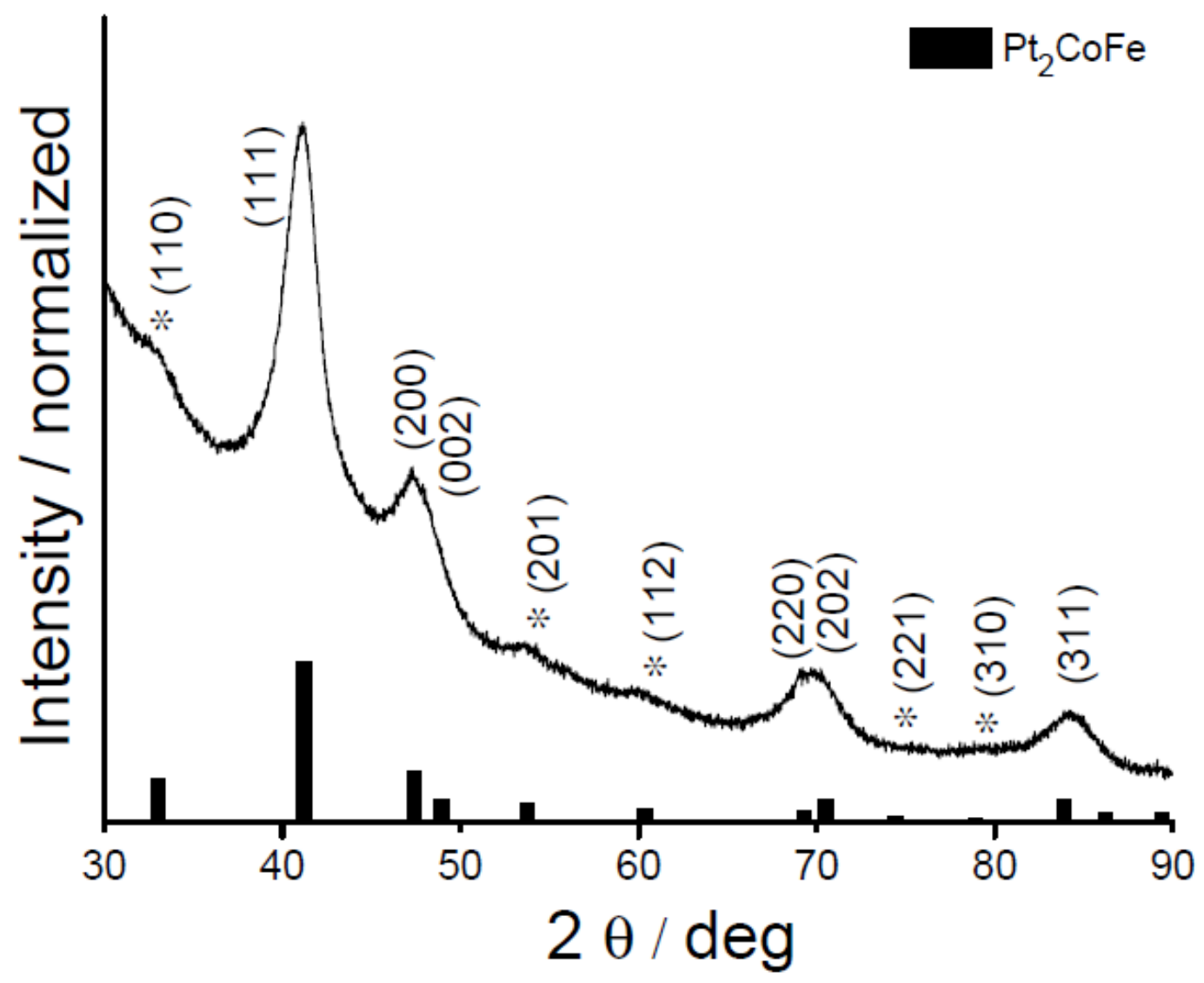

Figure 5.11: XRD spectra of carbon supported $\mathrm{Pt}_{2} \mathrm{CoFe}$, The lines on the bottom indicate the XRD peak positions of the corresponding intermetallic phase of the $\mathrm{Pt}_{2} \mathrm{CoFe}$ (PDF card \#01-077-7477). Peak positions for the ordered intermetallic phase of $\mathrm{Pt}_{2} \mathrm{CoFe}$ are marked with *. The full width and half maximum indicate small domain sizes of approximately $5 \mathrm{~nm}$.

The XRD of the transferred sample is shown in figure 5.11. The only peaks observed 
are indexed by the ordered cubic structure of $\mathrm{Pt}_{2} \mathrm{CoFe}$. No peaks corresponding to other Pt- Fe- or Co-containing compounds are present in the diffraction pattern. The observation of ordering peaks, such as the expected weak (110), (201) and (112) peaks, demonstrates that the desired tetragonal ordered intermetallic phase rather than the disordered cubic alloy phase of Pt-Ni was prepared.

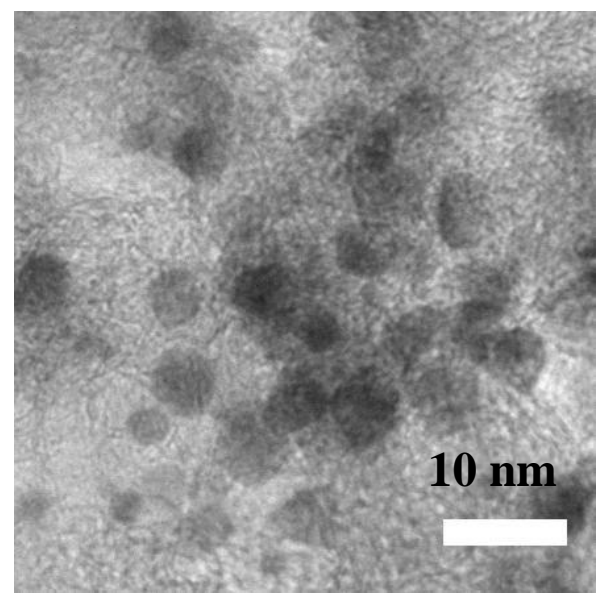

Figure 5.12: STEM-BF image of the annealed $\mathrm{Pt}_{2} \mathrm{CoFe}$ supported on carbon black

Shown in figure 5.12 is the STEM-BF image of the annealed $\mathrm{Pt}_{2} \mathrm{CoFe}$ supported on carbon black. Clearly, the particles are distributed on the carbon support without obvious aggregation and the particle sizes average about 3 to $6 \mathrm{~nm}$. 


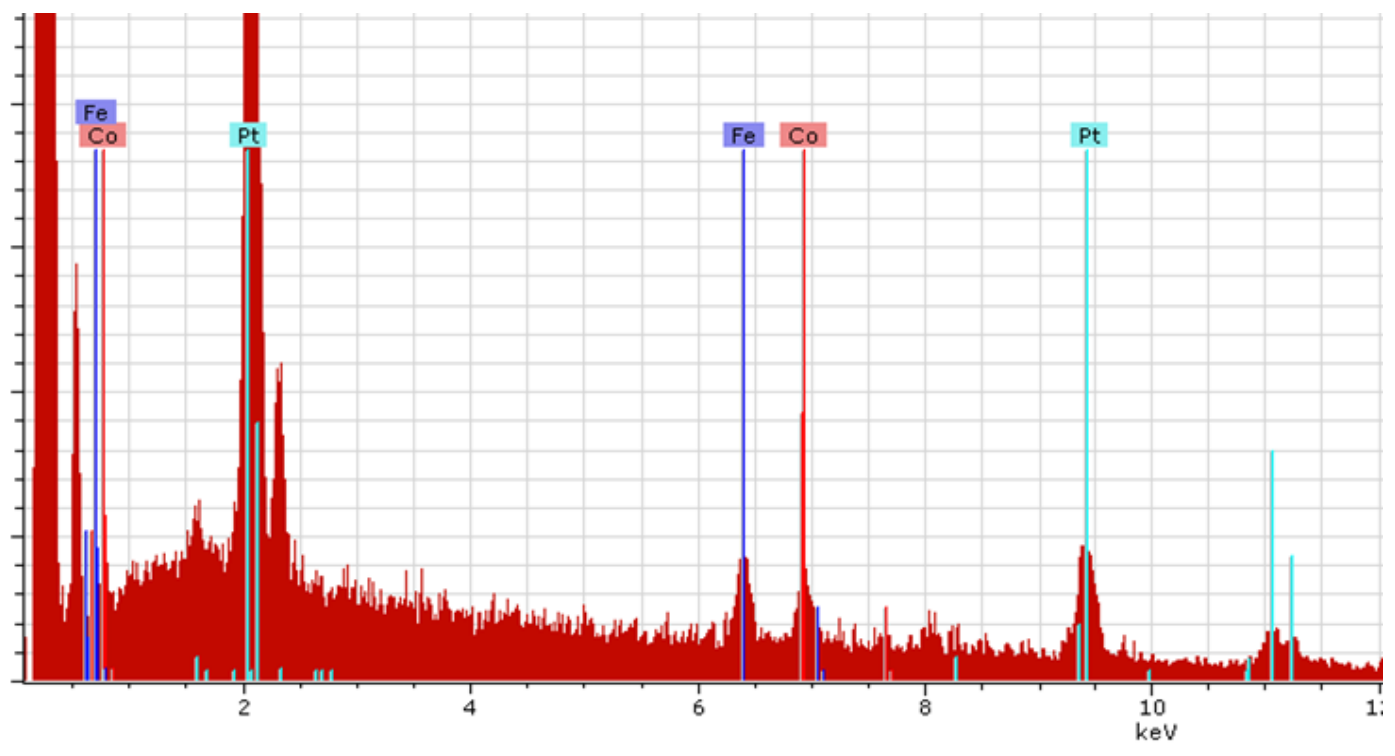

Figure 5.13: The EDX spectrum of $\mathrm{Pt}_{2} \mathrm{CoFe}$ sample

The average composition of an ensemble of nanoparticles in an area of $500 \mathrm{~nm}$ by 500 nm obtained by EDX spectroscopy for Pt:Co:Fe was typically 50:25:25 $( \pm 3 \%)$ and uniform throughout the sample (figure 5.13). STEM-EDX line scans of the PtNi nanoparticle confirms that the elements are distributed uniformly in all the examined nanoparticles (figure 5.14). 

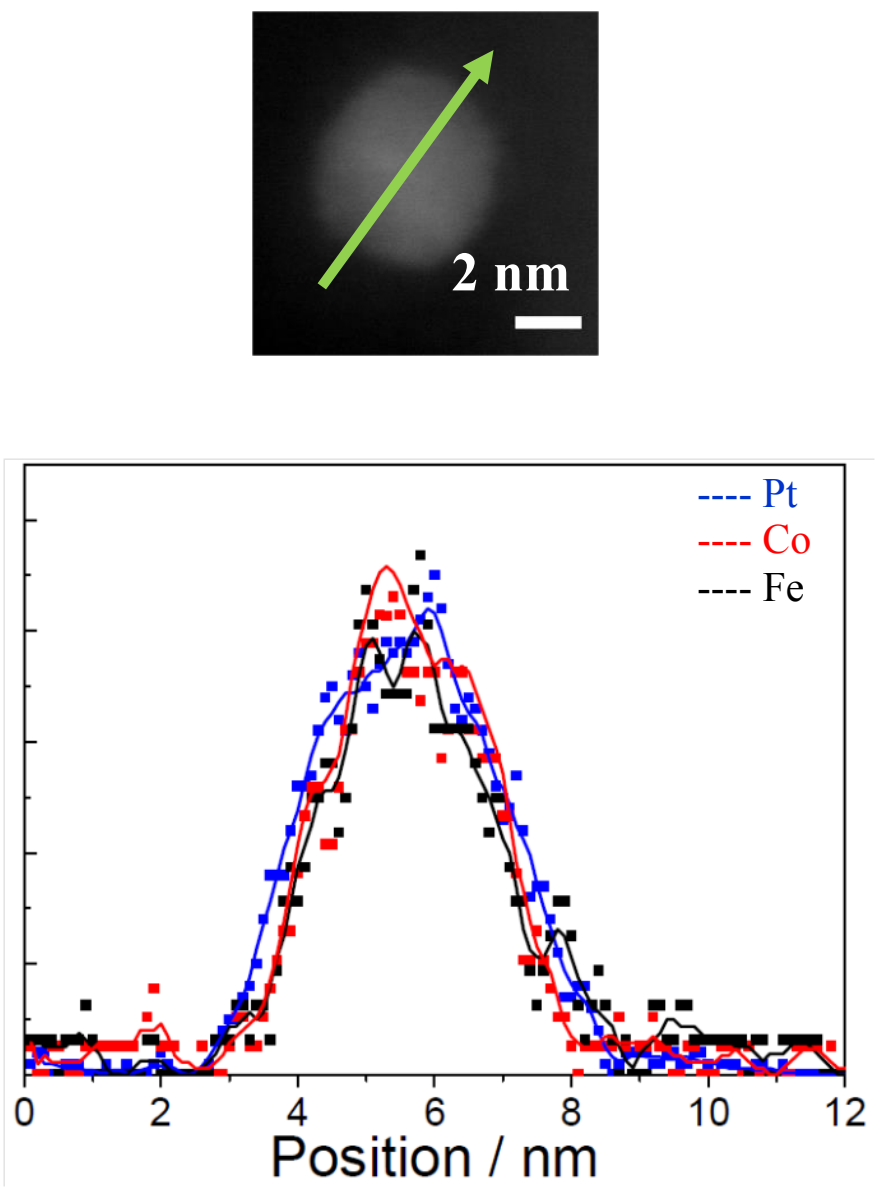

Figure 5.14: STEM-EDX line scans of $\mathrm{Pt}_{2} \mathrm{FeCo}$ nanoparticle show that the elements are distributed uniformly in the particles. The normalized best-fit lines are extrapolated by averaging adjacent points.

All measurements, including XRD, STEM, EDX and STEM-EDX line scans, confirm that the ordered intermetallic $\mathrm{Pt}_{2} \mathrm{CoFe}$ has been synthesized and transferred to support materials. 


\subsection{The synthesis of $\mathrm{Pt}_{3} \mathrm{~V}$ intermetallic nanoparticles}

The synthesis of $\mathrm{Pt}_{3} \mathrm{~V}-80 \mathrm{KCl}$ was carried as follows: $0.24 \mathrm{mmol} \mathrm{PtCl}_{4}, 0.08 \mathrm{mmol}$ $\mathrm{VCl}_{3}-3 \mathrm{THF}$ and $5.2 \mathrm{mmol} \mathrm{LiCl}$ were weighed out in an argon filled glovebox and dissolved in $20 \mathrm{~mL}$ THF by stirring. $7 \mathrm{~mL} \mathrm{KEt}_{3} \mathrm{BH}$ (1M Sigma-Aldrich) were mixed with THF to form a $10 \mathrm{~mL}$ solution. Then the precursor solution was drawn up into a syringe and injected into the reducing agent solution under vigorous stirring. The sample was dried under vacuum until most of the THF was evaporated. Hexanes were then added to precipitate the $\mathrm{Np}-\mathrm{KCl}$ powders. The sample was subsequently washed three times with a mixture solution of THF and hexanes without contacting air. The product was then dried under vacuum for 2 hours and transferred to the Ar glovebox. $\mathrm{The}_{\mathrm{Pt}} \mathrm{V}-80 \mathrm{KCl}$ powder was then placed into silica tubes, which were then transferred to a Schlenk / vacuum line and sealed under vacuum. The sample was then heated to $600{ }^{\circ} \mathrm{C}$ at a rate of $100{ }^{\circ} \mathrm{C} /$ hour and annealed at $600{ }^{\circ} \mathrm{C}$ for 10 hours and cooled to $300{ }^{\circ} \mathrm{C}$ at $30^{\circ} \mathrm{C} /$ hour. The samples were then transferred to Vulcan carbon support

using ethylene glycol as transfer agent. The resultant powder was then resealed in a silica vacuum tube and heated to $700{ }^{\circ} \mathrm{C}$ at a rate of $100{ }^{\circ} \mathrm{C} /$ hour and annealed at 700 ${ }^{\circ} \mathrm{C}$ for 24 hours and cooled to $300{ }^{\circ} \mathrm{C}$ at $30^{\circ} \mathrm{C} /$ hour and then cooled to room temperature.

The XRD, STEM and EDX were then conducted to demonstrate the intermetallic structure. 


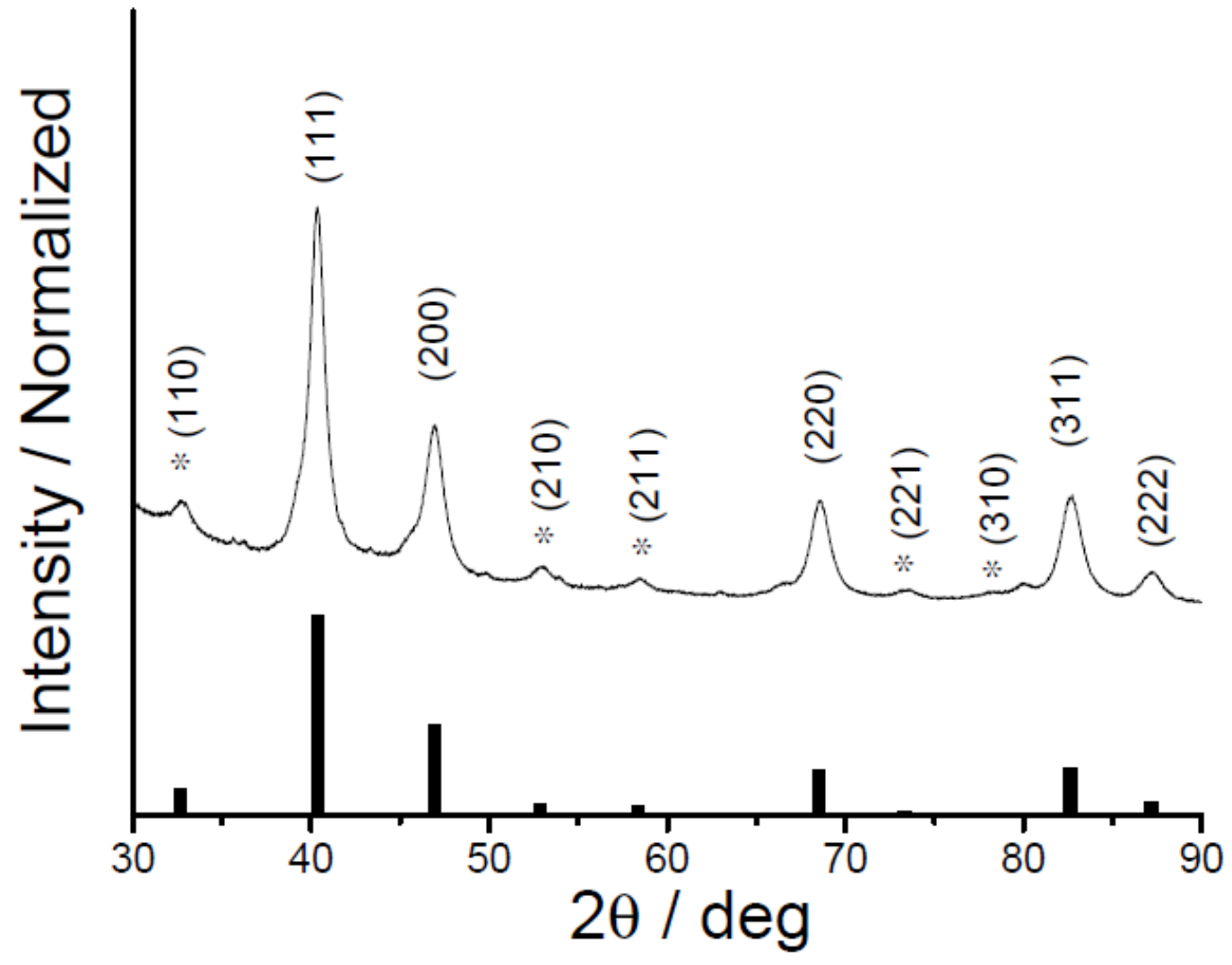

Figure 5.15: XRD spectra of carbon supported $\mathrm{Pt}_{3} \mathrm{~V}$, The lines on the bottom indicate the XRD peak positions of the corresponding intermetallic phase of the $\mathrm{Pt}_{3} \mathrm{~V}$ (PDF card \#04-001-3612). Peak positions for the ordered intermetallic phase of $\mathrm{Pt}_{3} \mathrm{~V}$ are marked with *.

The XRD of the transferred sample is shown in figure 5.15. The only peaks observed 
are indexed by the ordered cubic structure of $\mathrm{Pt}_{3} \mathrm{~V}$. No peaks corresponding to $\mathrm{Pt}, \mathrm{V}$, other intermetallics in the Pt-V phase diagram (such as the 1:1 PtV intermetallic phase), or any other Pt- or V-containing compounds are present in the diffraction pattern. The observation of ordering peaks, such as the expected weak (110), (210) and (211) peaks, demonstrates that the desired ordered intermetallic phase rather than the disordered alloy phase of Pt-V was prepared.

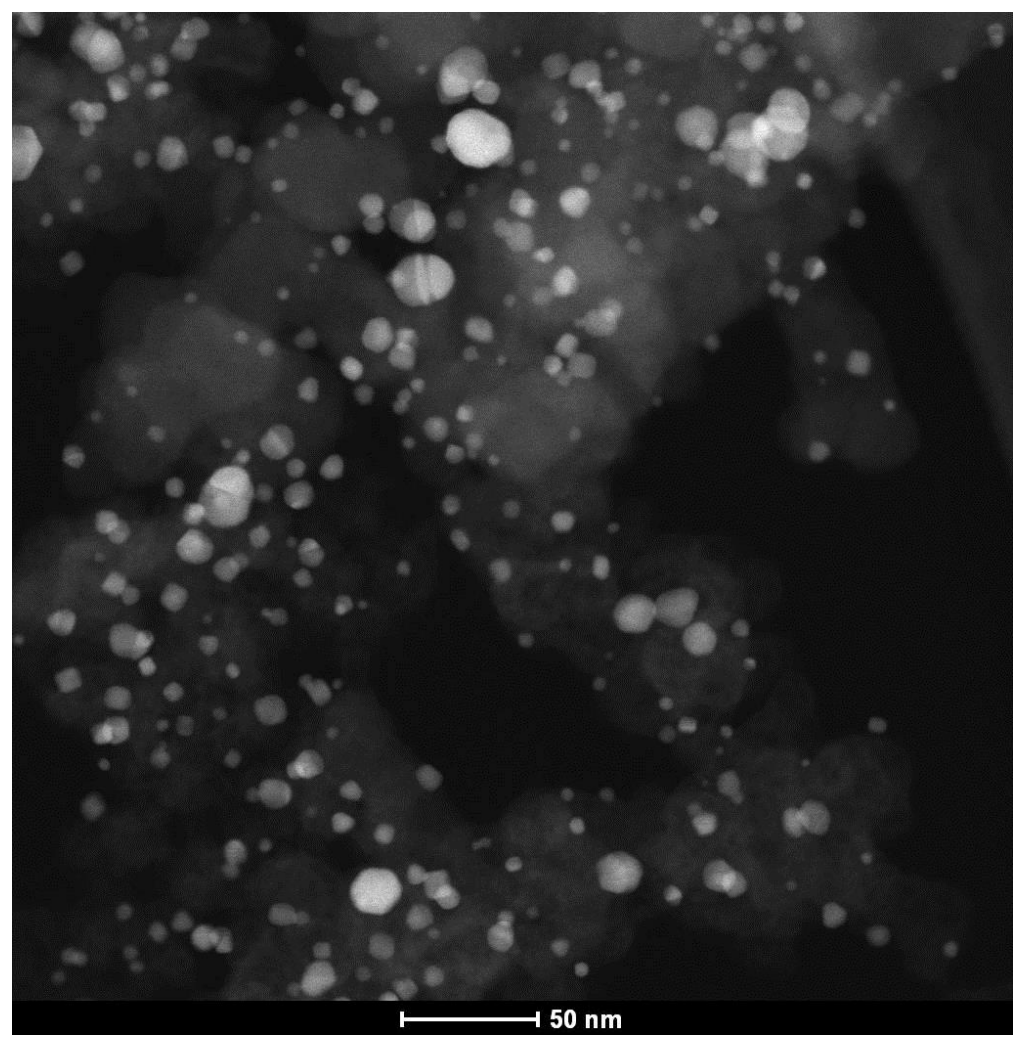

Figure 5.16: STEM-HAADF image of the annealed $\mathrm{Pt}_{3} \mathrm{~V}$ supported on carbon black 
Shown in figure 5.16 is the STEM-HAADF image of the annealed $\mathrm{Pt}_{3} \mathrm{~V}$ supported on carbon black. Clearly, the particles are distributed on the carbon support without obvious aggregation, but the higher annealing temperature of $700{ }^{\circ} \mathrm{C}$ has led to larger particle sizes, some on the order of $25 \mathrm{~nm}$.

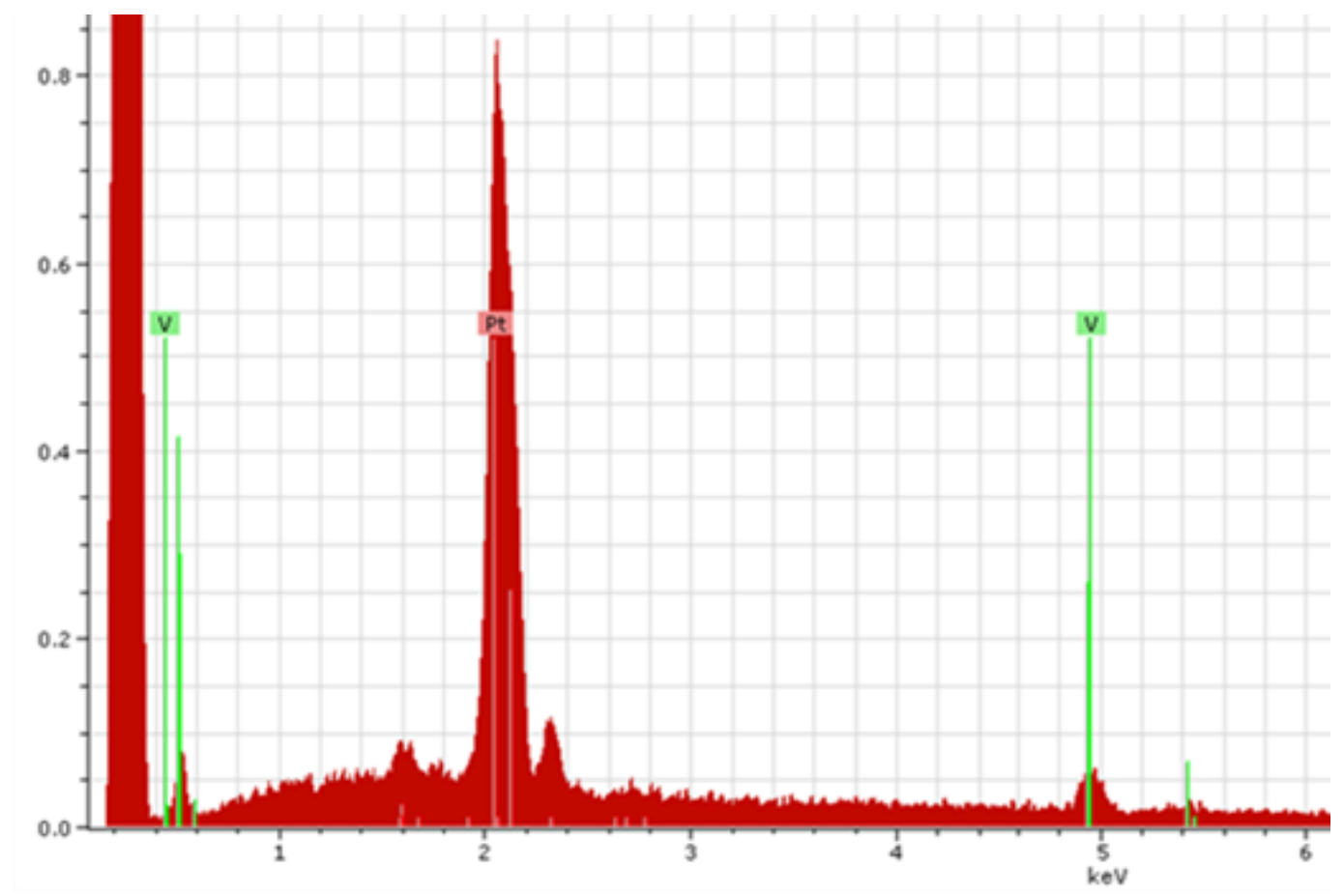

Figure 5.17: The EDX spectrum of $\mathrm{Pt}_{3} \mathrm{~V}$ sample

The average composition of an ensemble of nanoparticles in an area of $500 \mathrm{~nm}$ by 500 nm obtained by EDX spectroscopy for Pt:V was typically 75:25 ( $\pm 3 \%)$ and uniform throughout the sample (figure 5.17).

All measurements, including XRD, STEM and EDX confirm that using this $\mathrm{Np}-\mathrm{KCl}$ method, the ordered intermetallic $\mathrm{Pt}_{3} \mathrm{~V}$ can be synthesized and transferred to support materials. 


\subsection{The synthesis of Pt 3 Ti intermetallic nanoparticles}

The synthesis of $\mathrm{Pt}_{3} \mathrm{Ti}-80 \mathrm{KCl}$ was carried as follows: $0.24 \mathrm{mmol} \mathrm{PtCl}_{4}, 0.08 \mathrm{mmol}$ $\mathrm{TiCl}_{4}-2 \mathrm{THF}$ and $5.12 \mathrm{mmol} \mathrm{LiCl}$ were weighed out in an argon filled glovebox and dissolved in $20 \mathrm{~mL}$ THF by stirring. $7 \mathrm{~mL} \mathrm{KEt}_{3} \mathrm{BH}$ (1M Sigma-Aldrich) were mixed with THF to form a $10 \mathrm{~mL}$ solution. Then the precursor solution was drawn up into a syringe and injected into the reducing agent solution under vigorous stirring. The sample was dried under vacuum until most of the THF was evaporated. Hexanes were then added to precipitate the $\mathrm{Np}-\mathrm{KCl}$ powders. The sample was subsequently washed three times with a mixture solution of THF and hexanes without contacting air. The product was then dried under vacuum for 2 hours and transferred to the Ar glovebox. The $\mathrm{Pt}_{3} \mathrm{Ti}-80 \mathrm{KCl}$ powder was then placed into silica tubes, which were then transferred to a Schlenk / vacuum line and sealed under vacuum. The sample was then heated to $600{ }^{\circ} \mathrm{C}$ at a rate of $100{ }^{\circ} \mathrm{C} /$ hour and annealed at $600{ }^{\circ} \mathrm{C}$ for 10 hours and cooled to $300{ }^{\circ} \mathrm{C}$ at $30^{\circ} \mathrm{C}$ / hour. The samples were then transferred to Vulcan carbon support using ethylene glycol as transfer agent. The resultant powder was then resealed in a vacuum tube and heated to $700{ }^{\circ} \mathrm{C}$ at a rate of $100{ }^{\circ} \mathrm{C}$ / hour and annealed at $700{ }^{\circ} \mathrm{C}$ for 24 hours and cooled to $300{ }^{\circ} \mathrm{C}$ at $30^{\circ} \mathrm{C}$ / hour and then cooled to room temperature.

XRD, STEM and EDX measurements were then conducted to demonstrate the intermetallic structure. 


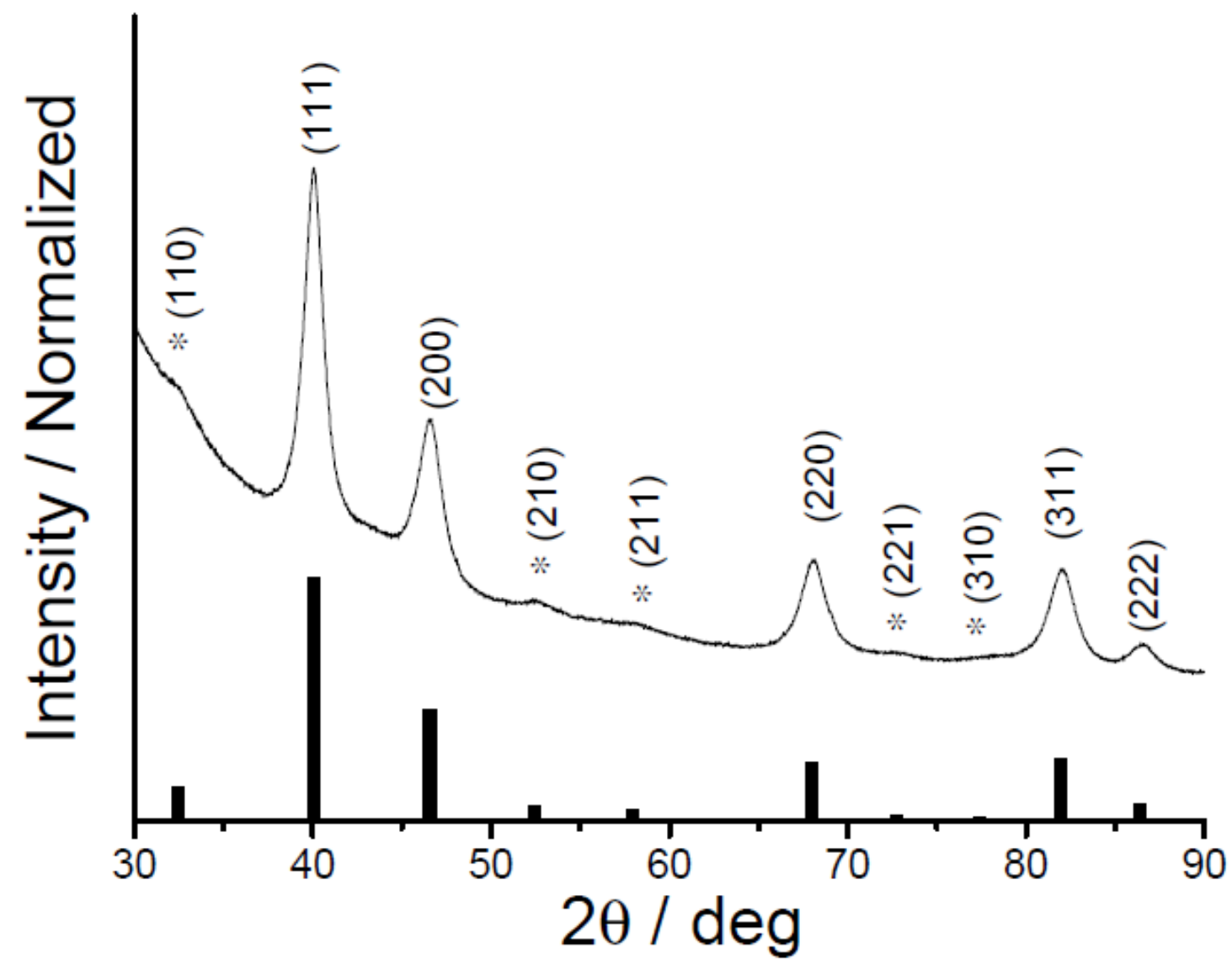

Figure 5.18: XRD spectra of carbon supported $\mathrm{Pt}_{3} \mathrm{Ti}$, The lines on the bottom indicate the XRD peak positions of the corresponding intermetallic phase of the $\mathrm{Pt}_{3} \mathrm{Ti}$ (PDF card \#03-065-3259). Peak positions for the ordered intermetallic phase of $\mathrm{Pt}_{3} \mathrm{Ti}$ are marked with *.

The XRD of the transferred sample is shown in figure 5.18. The only peaks observed 
are indexed by the ordered cubic structure of $\mathrm{Pt}_{3} \mathrm{Ti}$. No peaks corresponding to $\mathrm{Pt}$, $\mathrm{Ti}$, or other intermetallics in the Pt-Ti phase diagram (such as the 1:1 PtTi intermetallic phase), or any other Pt- or Ti-containing compounds are present in the diffraction pattern. The observation of ordering peaks, such as the expected weak (110), (210) and (211) peaks, demonstrates that the desired ordered intermetallic phase rather than the disordered alloy phase of Pt-Ti was prepared.

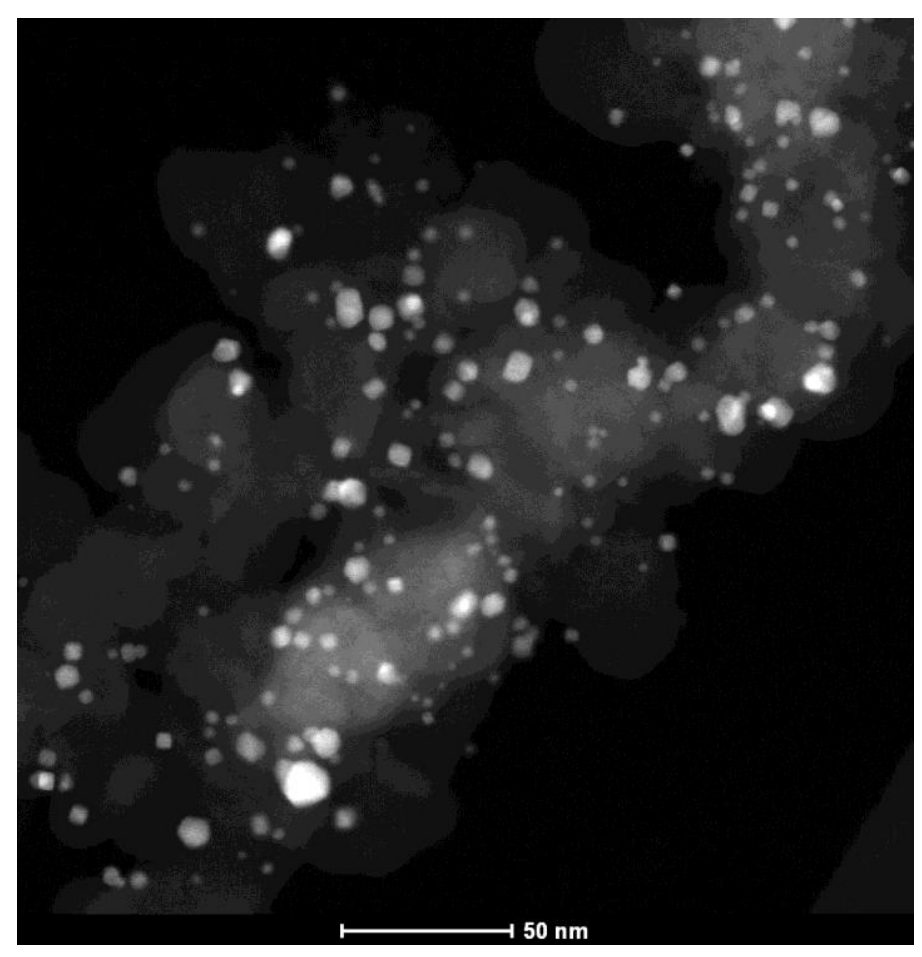

Figure 5.19: STEM-HAADF image of the annealed $\mathrm{Pt}_{3} \mathrm{Ti}$ supported on carbon black

Shown in figure 5.19 is the STEM-HAADF image of the annealed $\mathrm{Pt}_{3} \mathrm{Ti}$ supported on carbon black. Clearly, the particles are distributed on the carbon support without obvious aggregation and as expected the particle sizes are all below $10 \mathrm{~nm}$. 


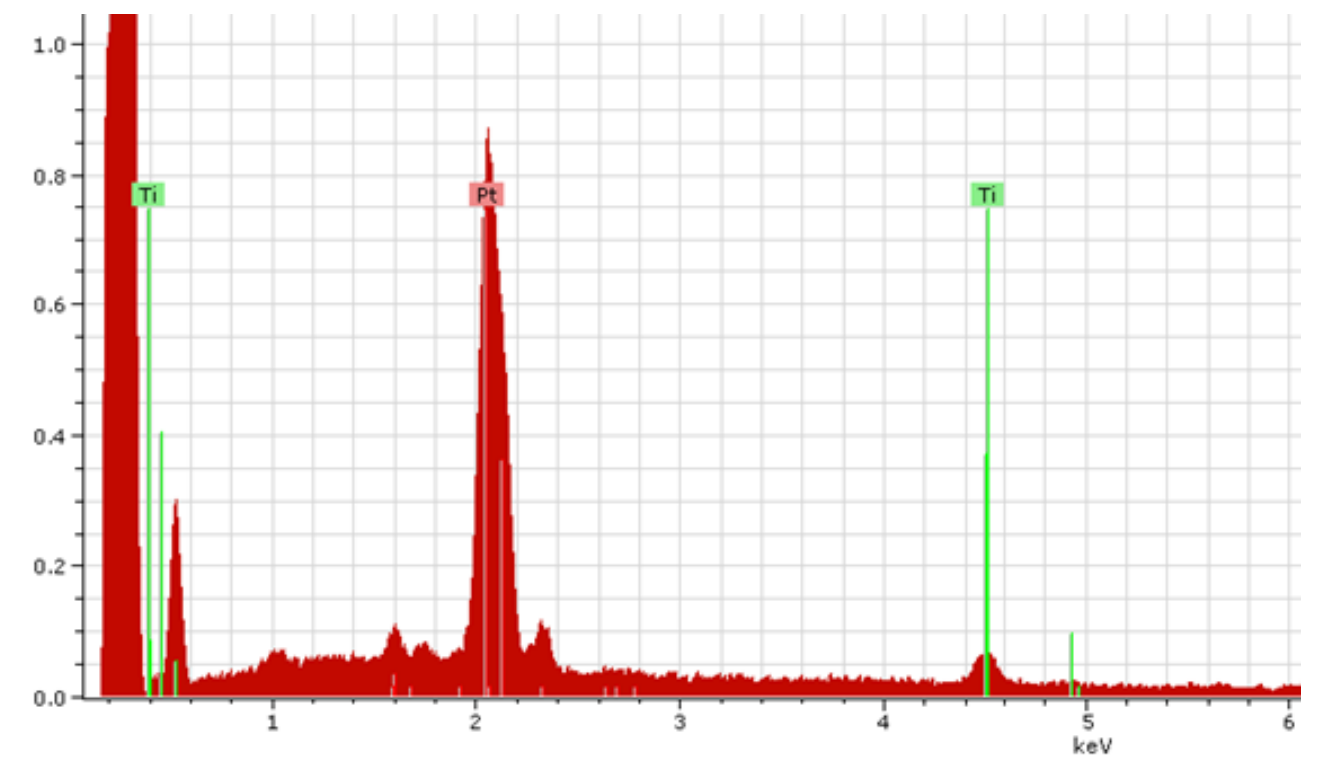

Figure 5.20: The EDX spectrum of $\mathrm{Pt}_{3} \mathrm{Ti}$ sample

The average composition of an ensemble of nanoparticles in an area of $500 \mathrm{~nm}$ by 500 nm obtained by EDX spectroscopy for Pt:Ti was typically 75:25 $( \pm 3 \%)$ and uniform throughout the sample (figure 5.20).

All measurements, including XRD, STEM and EDX confirm that using this $\mathrm{Np}-\mathrm{KCl}$ method, the ordered intermetallic $\mathrm{Pt}_{3} \mathrm{Ti}$ can be synthesized and transferred to support materials.

Interestingly, even though we could prepare $\mathrm{Pt}_{3} \mathrm{M}$ phases for $\mathrm{M}=\mathrm{Ti}, \mathrm{V} . \mathrm{Cr}$ and $\mathrm{Mn}$, Minh Nguyen, a graduate student in the group, was only able to synthesize the 
tetragonal PtM (1:1) phase for PtMn. This may be due to the extreme oxophilicity of $\mathrm{Ti}, \mathrm{V}$ and $\mathrm{Cr}$ and the relatively high mole fraction of these metals in the product. We hypothesize that trace amounts of air or water vigorously react with these metals at this composition, even at room temperature.

The synthesis of non-Pt based intermetallics using the $\mathrm{KCl}$ matrix method is just now beginning to be explored by Doug DeSario in the DiSalvo group. For ordered intermetallics to form, elements that are from different parts of the periodic table should be utilized ${ }^{15}$ It would be of interest in the future to show that phases like $\mathrm{AgPt} 5 \mathrm{Si}^{16}, \mathrm{Bi}_{4} \mathrm{Mn}_{5} \mathrm{Pd}_{2}{ }^{17}, \mathrm{Bi} 4 \mathrm{Cu} 4 \mathrm{Mn}_{3}{ }^{17}, \mathrm{NbRu}_{3} \mathrm{C}^{18}, \mathrm{MnNi}_{2} \mathrm{Sn}^{19}$, and $\mathrm{CuAuSn}_{2}{ }^{20}$ can be prepared

\subsection{Conclusion}

We have extended the Np-KCl matrix method to other Pt based nanoparticle systems. A wide variety of reactants based on binary or ternary chloride metal salts can be purchased or synthesized and are soluble in THF. Upon reduction with $\mathrm{KBEt}_{3} \mathrm{H}$, the resultant metal nanoparticles are trapped in $\mathrm{KCl}$. To achieve ordering and homogeneity, the $\mathrm{Np}-\mathrm{KCl}$ product need to be $\mathrm{n}$ annealed at temperatures up to 400 600 or even $700{ }^{\circ} \mathrm{C}$. Mn has an electronegativity of 1.5 , which makes it more difficult to reduce than $\mathrm{Fe}$ (electronegativity of 1.8). Using the method provided here, the 
ordered intermetallic form of $\mathrm{Pt}_{3} \mathrm{Mn}$ has been synthesized, while $\mathrm{PtMn}$ has been prepared by Minh Nguyen. Similarly, bi- and tri-metallic nanoparticles of PtNi and $\mathrm{Pt}_{2} \mathrm{CoFe}$ with a tetragonal ordered intermetallic structure were also synthesized and transferred to Vulcan carbon support using this method. To further demonstrate the generality, intermetallic compounds of $\mathrm{Pt}$ with the early oxophilic transition metals (Ti, V, Cr) were also synthesized as the most $\mathrm{Pt}$ rich composition $\left(\mathrm{Pt}_{3} \mathrm{M}\right)$ using this $\mathrm{Np}-\mathrm{KCl}$ method. All these results suggest that this method is applicable to a wider variety of transition metals and ordered structure types. The detailed experimental procedures can also be found in our recent papers ${ }^{21,22}$.

Acknowledgements: For the work in this chapter, I have received continuous help form Yingchao Yu for the TEM/STEM analysis and the DiSalvo Group specifically graduate students Minh Nguyen, Doug DeSario and my undergraduate student Kathryn Newton for the discussion and collaborate work of the synthesis using the nanoparticle- $\mathrm{KCl}$ method.

\section{REFERENCES}

(1) Goedkoop, J. A.; Andresen, A. F. Acta Crystallogr 1954, 7, 672.

(2) Wiberg, E.; Henle, W.; Bauer, R. Z Naturforsch B 1951, 6, 393.

(3) Zhao, J. W.; Ding, H.; Tian, X. F.; Zhao, W. J.; Hou, H. L. Chinese J Chem Phys 2008, 21, 569. 
(4) Er, S.; Tiwari, D.; de Wijs, G. A.; Brocks, G. Phys Rev B 2009, 79.

(5) Smithson, H.; Marianetti, C. A.; Morgan, D.; Van der Ven, A.; Predith, A.; Ceder, G. Phys Rev B 2002, 66.

(6) Fredrickson, D. R.; Hubbard, W. N.; Nuttall, R. L.; Flotow, H. E. J Phys Chem-Us 1963, 67, 1506.

(7) Guo, Q. T.; Kleppa, O. J. J Alloy Compd 1998, 266, 224.

(8) Bardi, U.; Ross, P. N. J Vac Sci Technol A 1984, 2, 1461.

(9) Guo, Q. T.; Kleppa, O. J. J Alloy Compd 1994, 205, 63.

(10) Watson, R. E.; Bennett, L. H. Calphad 1984, 8, 307.

(11) Meschel, S. V.; Nash, P.; Chen, X. Q. J Alloy Compd 2010, 492, 105.

(12) Srikrish.V; Ficalora, P. J. Metall Trans 1974, 5, 1471.

(13) Luo, W.; Flanagan, T. B.; Clewley, J. D.; Dantzer, P. Metall Trans A $1993,24,2623$.

(14) Watanabe, K.; Matsuyama, M.; Ashida, K.; Miyake, H. J Vac Sci Technol A 1989, 7, 2725.

(15) Villars, P.; Cenzual, K.; Daams, J.; Chen, Y.; Iwata, S.; J Alloy Compd $\mathbf{2 0 0 4}, 367,167$

(16) El-Boragy, M.; Schubert, K.; Z Metallkd 1970, 61, 579.

(17) Street, G. B.; Suits, J. C.; Lee, K.; Solid State Commun 1974, 14, 33.

(18) Holleck, H.; Mettall 1983, 37, 475.

(19) Kuz'min, R. N.; Ibraimov, N. S.; Zhdanov, G. S.; Zhurnal Eksperimental'noi i Teoreticheskoi Fiziki 1966, 50, 330.

(19) Leonard, B. M.; Schaak, R. E.; J Am Chem Soc 2006, 128, 1475.

(21) Chen, H.; Wang, D. L.; Yu, Y. C.; Newton, K. A.; Muller, D. A.; 
Abruna, H.; DiSalvo, F. J. J Am Chem Soc 2012, 134, 18453.

(22) Chen, H.; Yu, Y.; Xin, H.; Newton, K. A.; Holtz, M. E.; Wang, D.; Muller, D. A.; Abruña, H.; DiSalvo, F. J., Chem Mater 2013, 25, 1436. 